\title{
Uncertainty Modeling for Structural Control Analysis and Synthesis
}

\author{
Mark E. Campbell and Edward F. Crawley \\ January 1996 \\ SERC\#2-96
}

(Under the sponsorship of NASA)

This report is based on the unaltered thesis of Mark E. Campbell submitted to the Department of Aeronautics and Astronautics in partial fulfillment of the requirements for the degree of Doctor of Philosophy at the Massachusetts Institute of Technology. 


\begin{abstract}
The development of an accurate model of uncertainties for the control of structures that undergo a change in operational environment, based solely on modeling and experimentation in the original environment is studied. The application used throughout this work is the development of an on-orbit uncertainty model based on ground modeling and experimentation. A ground based uncertainty model consisting of mean errors and bounds on critical structural parameters is developed. The uncertainty model is created using multiple data sets to observe all relevant uncertainties in the system. The Discrete Extended Kalman Filter is used as an identification/parameter estimation method for each data set, in addition to providing a covariance matrix which aids in the development of the uncertainty model. Once ground based modal uncertainties have been developed, they are localized to specific degrees of freedom in the form of mass and stiffness uncertainties. Two techniques are presented: a matrix method which develops the mass and stiffness uncertainties in a mathematical manner; and a sensitivity method which assumes a form for the mass and stiffness uncertainties in macroelements and scaling factors. This form allows the derivation of mass and stiffness uncertainties in a more physical manner. The mass and stiffness uncertainties of the ground based system are then mapped onto the on-orbit system, and projected to create an analogous on-orbit uncertainty model in the form of mean errors and bounds on critical parameters. The Middeck Active Control Experiment is introduced as experimental verification for the localization and projection methods developed. In addition, closed loop results from on-orbit operations of the experiment verify the use of the uncertainty model for control analysis and synthesis in space.
\end{abstract}




\section{Acknowledgments}

This research was supported by the NASA In-Step and NASA LaRC CSI Office, with Mr. Gregory Stover and Dr. Jerry Newsom as contract monitors, under contracts NAS1-18690 NAS1-19622. 


\section{Contents}

1 Introduction $\quad 13$

1.1 Motivation ...................... 13

1.2 Previous Work ... . . . . . . . . . . . . 15

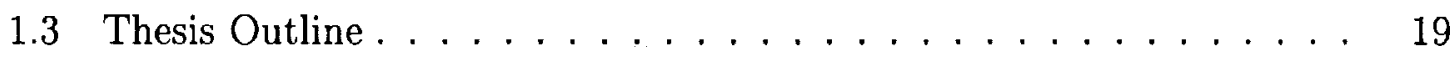

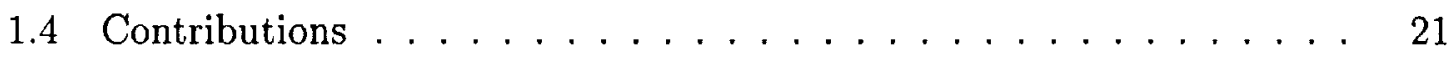

2 Discussion of Models and Hardware 23

2.1 Middeck Active Control Experiment (MACE) . . . . . . . . . 24

2.1.1 Modeling Process . . . . . . . . . . . . . . 25

2.1 .2 Hardware Description . . . . . . . . . . . . 28

$2.1 .3 \quad$ STS-67.............................. 29

2.1 .4 Finite Element Model ............... 31

2.2 Assessment of Modeling Uncertainties . . . . . . . . . . . . . . . . . . . . . . .

2.2.1 Physical Uncertainties . . . . . . . . . . . . . . 34

2.2.2 Modeling Uncertainties . . . . . . . . . . . . . . . . 37

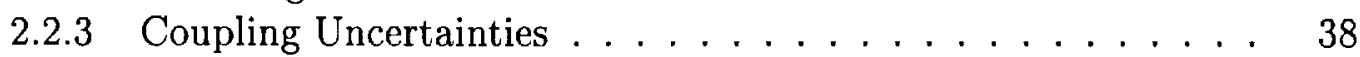

2.2 .4 Testing Uncertainties . . . . . . . . . . . . . . . . . . 42

2.2 .5 Nonlinearities . . . . . . . . . . . . . 42

2.2 .6 Summary ...................... 43

3 Identification of Nominal Uncertainty Model $\quad 45$

3.1 Testing for Uncertainty Identification . . . . . . . . . . . . 46

3.2 Discrete Extended Kalman Filter . . . . . . . . . . . . . . . . . . . . . . . . . . . 48

3.2.1 Finite Element Based DEKF . . . . . . . . . . . . 48

3.2 .2 Additional Dynamics . . . . . . . . . . . . . . 52

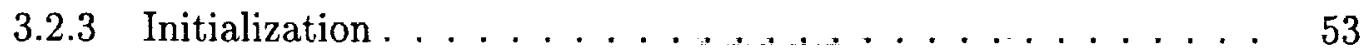

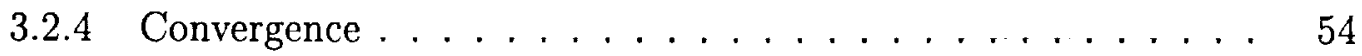

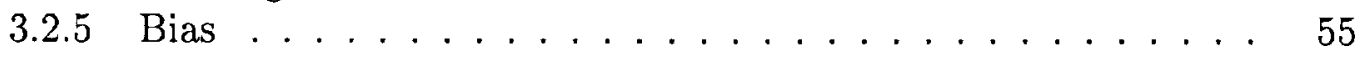

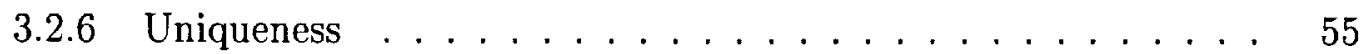

3.3 Uncertainty Model . . . . . . . . . . . . . . . . . . 56

3.4 MACE Results . . . . . . . . . . . . . . . . . . . . . . . . . . . . . .

3.4 .1 Testing ............................. 58

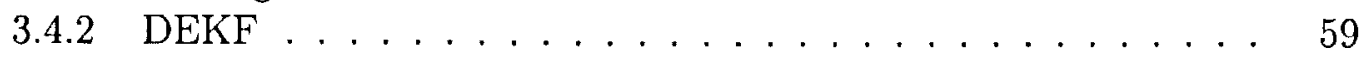

$3.4 .3 \quad 1-$ g Uncertainty Model . . . . . . . . . . . 63 
4 Uncertainty Localization and Projection $\quad 67$

4.1 Problem Formulation . . . . . . . . . . . . . . . . . 68

4.2 Matrix Method . . . . . . . . . . . . . . . . 71

4.2 .1 Uncertainty Localization . . . . . . . . . . . . . . 72

4.2 .2 Uncertainty Projection . . . . . . . . . . . . . 73

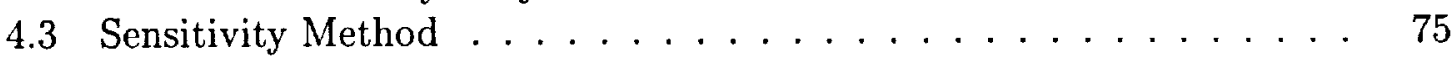

4.3.1 Uncertainty Localization . . . . . . . . . . . 75

4.3.2 Uncertainty Projection . . . . . . . . . . . . . 78

4.3.3 Macroelements . . . . . . . . . . . . . . . 79

4.4 Practical Algorithm . . . . . . . . . . . . . . . . . . . 83

4.5 Small Order Example . . . . . . . . . . . . . . . . . . . . 87

4.5.1 Case 1: Complete Measurements . . . . . . . . . . 91

4.5.2 Case 2: Partial Measurements . . . . . . . . . . . . 97

4.5.3 Case 3: Partial Measurements . . . . . . . . . . . . . 100

5 Experimental Verification $\quad 107$

5.1 MACE Configuration I $1-\mathrm{g}$ to $0-\mathrm{g} \ldots \ldots \ldots \ldots \ldots \ldots \ldots$

5.1 .1 Matrix Method .................. . . 110

5.1 .2 Sensitivity Method . . . . . . . . . . . . . . 111

${ }^{5} .2$ MACE $0-\mathrm{g}$ Configuration I to Configuration II $\ldots \ldots \ldots \ldots$

6 Controller Synthesis and Analysis 131

6.1 Control of Uncertain Systems . . . . . . . . . . . . . . . 132

6.1.1 Controller Synthesis . . . . . . . . . . . . . . . 133

6.1 .2 Controller Analysis . . . . . . . . . . . . . . . . 134

6.1.3 Utilization of the Uncertainty Model . . . . . . . . . 135

6.2 MACE $0-$ g Results . . . . . . . . . . . . . . . . 136

6.2 .1 Controller Synthesis . . . . . . . . . . . . 136

6.2 .2 Controller Analysis . . . . . . . . . . . . . . . 137

6.2.3 Utilization of the Uncertainty Model . . . . . . . . . . . 139

6.2 .4 Closed Loop Results . . . . . . . . . . . . . . . . . 142

6.2.5 Analysis of Uncertainty Model . . . . . . . . . . . . . . 144

7 Conclusions 147

7.1 Summary . . . . . . . . . . . . . . . . . 147

7.2 Contributions . . . . . . . . . . . . . . . 151

7.3 Recommendations . . . . . . . . . . . . . . . . . 152

$\begin{array}{ll}\text { References } & 155\end{array}$ 


\section{List of Figures}

1.1 Development of control design and uncertainty models for $0-\mathrm{g}$. . . . 15

2.1 MACE Flight Model Test Article . . . . . . . . . . . . . . . . 25

2.2 Modeling and Control approach for MACE . . . . . . . . . . 26

2.3 MACE FM nodal numbering in the 1-g finite element model. . . . . . 32

2.4 Sample beam element and reference axes and notation. . . . . . . 35

2.5 Nodal and distributed loads on a beam element in gravity. . . . . . . 40

3.1 FEM, updated, and data transfer functions for a sample identification of the DEKF on MACE. . . . . . . . . . . . . . 61

3.2 Time histories for the recursive DEKF, showing the convergence of frequency and damping ratio estimates. . . . . . . . . . . 62

3.3 Development of 1-g control design and uncertainty models. . . . . . . 64

4.1 Uncertainty model development problem. . . . . . . . . . . . 71

4.2 Three distinct macroelements. . . . . . . . . . . . . . . 80

4.3 Three step procedure for uncertainty localization using different types of macroelements. . . . . . . . . . . . . . . . . . . 84

4.4 Example of the reduction of macroelements using the weighted least

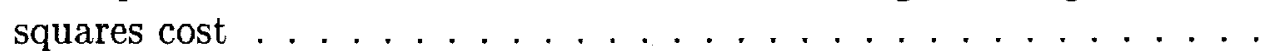

4.5 Flowchart for the practical implementation of the uncertainty localization and projection methods. . . . . . . . . . . . 88

4.6 Small order example: cantilevered "L" beam . . . . . . . . . . . 89

4.7 Matrix method for Case 1A using complete measurements. . . . . . . 93

4.8 Weighted least squares cost showing the possible elimination of macroelements. . . . . . . . . . . . . . . . 95

4.9 Sensitivity method for Case 1A using complete measurements. . . . . 96

4.10 Localization and projection of variance uncertainties to predict frequency bounds for Case 1B using complete measurements. . . . . . . 98

4.11 Matrix Method for Case 2 using partial measurements. . . . . . . . . 100

4.12 Sensitivity Method for Case 2 using partial measurements. . . . . . . 101

4.13 Sensitivity Method for Case 3 using partial measurements and one iteration. . . . . . . . . . . . . . . . 103

4.14 Frequency results using multiple itcrations of the Sensitivity Method for Case 3 using partial measurements. (-) - updated or projected frequencies, $(x)$ - simulated mean frequency, $(0)$ - actual frequency. . 104 
4.15 Sensitivity Method for Case 3 using partial measurements and 5 iterations.

5.1 Two $0-\mathrm{g}$ configurations of the MACE test article. . . . . . . . 108

5.2 MACE Configuration I $1-\mathrm{g}$ to $0-\mathrm{g}$ projection using the matrix method with eigenvalue uncertainties.. . . . . . . . . . . . 112

5.3 MACE Configuration I $1-\mathrm{g}$ to 0 -g projection using the matrix method with eigenvalue and eigenvector uncertainties. . . . . . . . . . 113

5.4 MACE 1-g localized stiffness matrix mean error uncertainties. . . . . 116

5.5 MACE 1-g localized mass matrix mean error uncertainties. . . . . . . 118

5.6 MACE Configuration I $1-\mathrm{g}$ to $0-\mathrm{g}$ projection using the sensitivity method. . . . . . . . . . . . . . . . . 119

5.7 MACE 0-g FEM, 0-g FEM shifted by projected mean errors, and three sets of data. . . . . . . . . . . . . . . . . 121

5.8 MACE 0-g Configuration I localized mean error uncertainties. . . . . 125

5.9 MACE 0-g Configuration I to Configuration II (C2) projection using the sensitivity method. . . . . . . . . . . . . 126

5.10 MACE 0-g Configuration II (C2) FEM, 0-g C2 FEM shifted by projected mean errors, and one set of data. . . . . . . . . . . . . . 128

5.11 MACE 0-g Configuration II localized uncertainties. . . . . . . . . . . 129

6.1 Interconnection of plant $G$, controller $K$, and uncertain block $\Delta$. . . 132

6.2 Types of controller synthesis and analysis using an uncertainty model 136

6.3 Interconnection of the open loop system $(G)$, compensator $(K)$, real parameter uncertainty $\left(\Delta_{0}\right)$, and weighting on the Sensitivity $(W)$, and the Sensitivity delta block $\left(\Delta_{S}\right) \ldots \ldots \ldots$. . . . . . . . 140

6.4 Example of the usage of the mixed- $\mu$ analysis tool, and the uncertainty model. . . . . . . . . . . . . . . . . . 141

6.5 MACE 0-g closed loop results using for LQG and SWLQG controllers. 142

6.6 MACE 0-g closed loop results using unshifted model/symmetric bounds (FEM) and shifted model/symmetric bounds (shifted). . . . . . 144

6.7 Mixed- $\mu$ analysis of a $5 \times 5 \mathrm{XYZ}$ controller using three types of uncertainty models derived using the sensitivity method. . . . . . . . . 146 


\section{List of Tables}

2.1 Breakdown of control protocols implemented on STS-67. . . . . . .

2.2 Nodal numbering and element descriptions for the MACE FM finite element model. . . . . . . . . . . . . . . . . . .

2.3 Frequency, damping ratio, and description of each mode retained in the finite element model for the MACE FM test article in 1-g and 0-g. (0-60 $\mathrm{Hz}$ range) . . . . . . . . . . . . . . .

2.4 Breakdown of mass and stiffness element change matrices for changes in physical parameters for a tubular beam element. . . . . . . . .

2.5 Table of uncertainties between structural hardware and models, along with the ability to capture them using identification and/or localization methods. . . . . . . . . . . . . . . . .

3.1 Set of test parameters and whether they are primarily used for the development of an uncertainty model, or finite element model updating. 47

3.2 Set of tests performed on MACE to observe modal uncertainties and update the finite element model. . . . . . . . . . . . . . . 59

3.3 MACE 1 -g uncertainty model. . . . . . . . . . . . . . . 65

4.1 Beam sectional and nodal properties for the small order example . . 89

4.2 Frequencies for the cantilevered and free-free configurations of the small order example . . . . . . . . . . . . . . . . . . 90 90

4.3 Test cases for the small order example. . . . . . . . . . . . 91

5.1 MACE Configuration I 1-g to $0-$ g summary. . . . . . . . . . . 109

5.2 MACE $1-\mathrm{g}$ to $0-\mathrm{g}$ changes . . . . . . . . . . . . . 120

5.3 MACE $0-\mathrm{g}$ Configuration I to Configuration II summary. . . . . . . . 123

5.4 MACE 0-g Configuration I to Configuration II changes . . . . . . 127 


\section{Chapter 1}

\section{Introduction}

\subsection{Motivation}

Practical control design for space structures typically requires a very accurate model of the dynamics, in addition to a robust control design procedure. Two common approaches exist for developing a model for designing on-orbit controllers: 1) a model of the structure is made prior to flight, and controllers are designed based upon this model, 2) an on-orbit identification is performed and controllers are designed based upon this measurement model. In this era of higher performing, low cost experiments, option (1) uses test heritage to increase performance and avoid catastrophic failure, while avoiding the costly on-orbit (re)design of the control system.

To develop the most accurate model possible for control design on a space structure prior to flight, extensive ground modeling and testing must be performed. Figure 1.1 shows this process schematically. A 1-g finite element model is developed using physical parameters and updated using open loop data to obtain good accuracy. Additional dynamics for sensors, actuators, time delays, etc. are appended to the finite element model to create an input-output model. Multiple identification experiments are performed, and the data is fit with even higher accuracy measurement models. By comparing the measurement models and input-output model, a 1-g uncertainty model consisting of mean errors and bounds on critical parameters is developed. A shifted input-output model can be created by shifting the parameters of the input- 
output model by the mean errors. Robust controllers can be designed on the shifted model, and evaluated using the parameter bounds from the 1-g uncertainty model.

A model of the on-orbit dynamics is created by removing the gravity effects and suspension degrees of freedom from the 1-g finite element model, and appending the appropriate additional dynamics. This is termed the $0-\mathrm{g}$ input-output model. A $0-\mathrm{g}$ uncertainty model is developed by projecting the mean errors and bounds from the 1-g uncertainty model into 0-g. This projection, which is one of the central ideas of this work, is accomplished using all of the information available, i.e. the $1-\mathrm{g}$ and $0-\mathrm{g}$ finite element models, and the 1-g uncertainty model. A $0-\mathrm{g}$ shifted input-output model can then be created in an analogous manner to the 1-g shifted input-output model. Robust control design in $0-\mathrm{g}$ can then be performed using the shifted inputoutput model, and evaluated using the predicted bounds on critical parameters of the system. In addition to developing accurate models of the on-orbit dynamics for control design prior to flight (shifted input-output and uncertainty model), modeling and control confidence have been developed from the ground based test heritage.

The updated finite element model and multiple identifications data sets in 1-g shown at the top of Figure 1.1 are assumed to be complete at the beginning of this work. The $0-\mathrm{g}$ finite element model is also available, given the removal of gravity and suspension degrees of freedom from the 1-g finite element model. The bottom of Figure 1.1 is the focus of the work in this thesis. The specific issue addressed in this work is, "Can accurate structural dynamic models and uncertainty models be created for control analysis and synthesis of structures in space, based solely on modeling and experimentation on the ground?"

The specific objectives of this thesis are as follows:

- Develop a 1-g uncertainty model based on a finite element model and sets of open loop identification data.

- Develop analytical techniques using the $1-\mathrm{g}$ and $0-\mathrm{g}$ finite element models and the 1-g uncertainty model, to accurately predict the $0-\mathrm{g}$ uncertainty model.

- Examine how the shifted model and uncertainty model can be used for control synthesis and analysis.

- Demonstrate these techniques experimentally. 


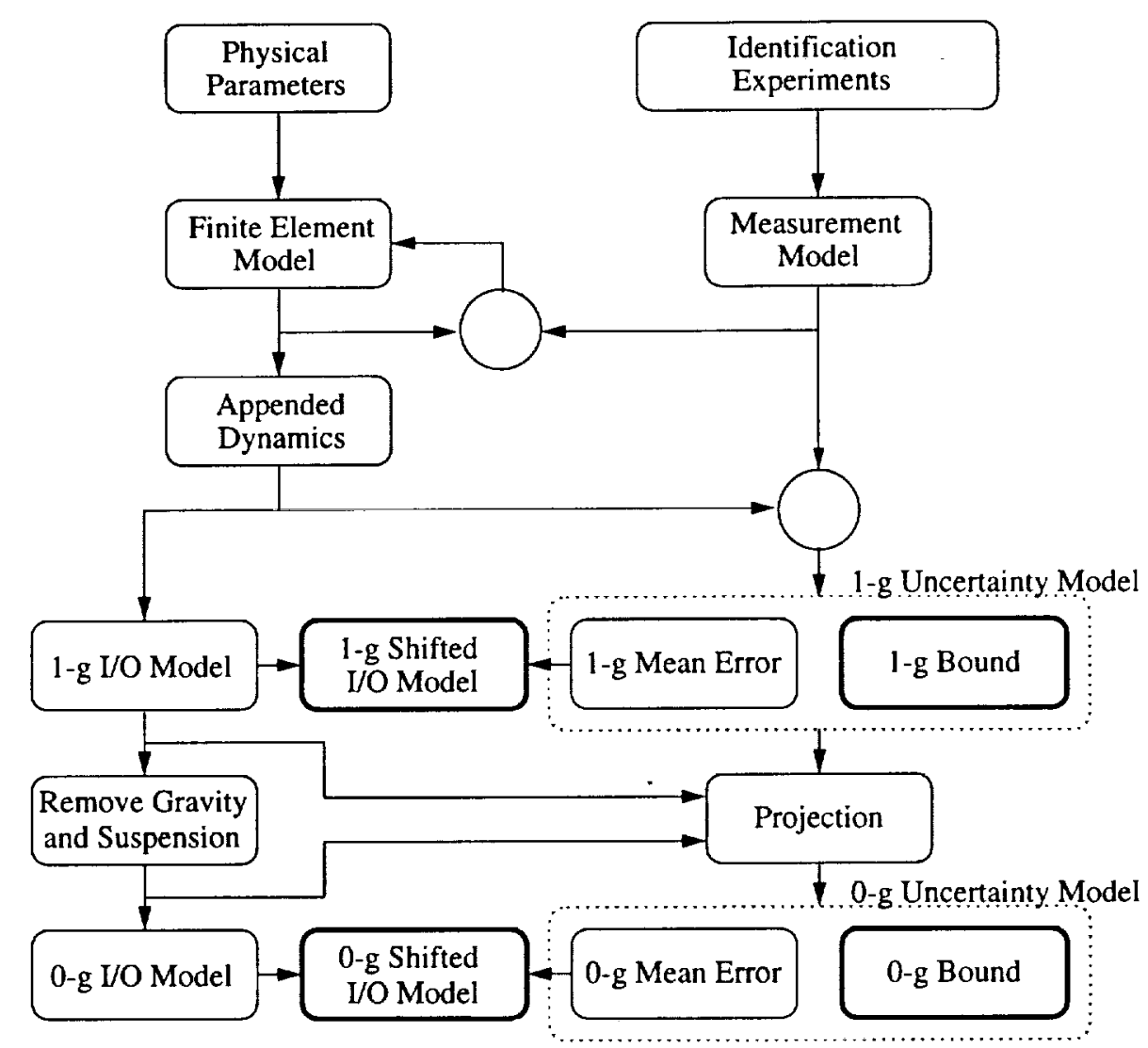

Figure 1.1: Development of control design and uncertainty models for $0-\mathrm{g}$.

\subsection{Previous Work}

Figure 1.1 shows that developing a good control design model and uncertainty model for $0-\mathrm{g}$ is a complex blending of many disciplines. These include finite element modeling and updating, system identification, probability analysis, and finite element error localization (for the uncertainty projection problem). Finite element modeling and updating are assumed to be complete, and are therefore not examined here. A summary of system identification techniques is given, although this is not intended to be an exhaustive listing, only a summary. The areas which are considered the main focus of this work, finite element error localization and stochastic analysis of models and hardware, are thoroughly examined.

System identification techniques, the term applied to predicting models from open loop data, have evolved from both the structural dynamics [1] and control systems [2] 
fields. While there are many algorithms which obtain very accurate fits to the data, many techniques create difficulties in using them for model updating and error localization. These difficulties include estimating the frequency, damping, and mode shape information from a model with repeated modes; estimating normal modes from measured complex modes; and the inherent coupling between the structural system and additional dynamics such as time delays, sensor dynamics, and closed loop servo system. There are a few methods, however, which parameterize the system in a manner such that modal identification is much easier. This includes easy parameterization of additional dynamics for the actuators and sensors, time delays, etc. A few methods have been developed, using both frequency domain [3-5] and time domain [6] data to overcome these problems.

Finite element model error localization techniques have become an area of intense research over the past 10 years. These techniques attempt to localize errors in the finite element model to specific degrees of freedom using measured frequencies and mode shapes from modal analysis, and an initial finite element model. A number of papers give excellent surveys of work in this area [7-9]. There are a few methods which localize the errors to specific degrees of freedom, but do not give an indication of how to update the system [10-12]. The most notable of these is the Coordinate Modal Assurance Criterion (COMAC) [10], where the errors are localized by examining the differences in the theoretical and experimental mode shapes at the measured degrees of freedom. The most complete solutions, however, localize and correct the model errors. These methods can be divided into four groups: Matrix Methods, Sensitivity Methods, Frequency Domain Methods, Dynamic Reaction Methods.

Matrix Methods work directly with the mass and stiffness matrices in localizing the errors [13-15]. Sidhu and Ewins [16] developed the most well known of these called the Error Matrix Method. A system is first reduced to the measured degrees of freedom using techniques such as Guyan reduction [17]. Errors in the flexibility matrix are identified, and then inverted to get the measured stiffness errors. Although this is one of the more practical methods because it attempts to keep the physical nature of the system, Gysin [18] showed that it is extremely dependent upon the 
choice of reduction technique.

Sensitivity Methods make use of the first order sensitivities of the modal parameters, i.e. the Jacobian. The mass and stiffness matrices are expressed as a function of design parameters $(E, I, \rho$, etc.) The first order sensitivities of the system can then be considered. Although many variations of this method have been presented [19-22], most depend on measurement of the mode shape at all degrees of freedom (or the experimental mode shape is expanded to the full mode shape.) The most well known variant is due to Lallement et al. [23-26]. In these papers, the problems with the method are addressed such as sensitivity problems, the choice of design parameters, the matching of mode shapes, and the use of partial information. Although not used by many of the researchers in this area, the most appealing aspect of this variant is that partial measurement of the mode shapes can still be used, without expansion.

Frequency Domain Methods utilize the frequency response functions, instead of the modal parameters in generating error matrices. These methods build an error matrix in the frequency domain that is not in the form of a correction to the mass or stiffness matrices. Many of the techniques are transformations of already known methods into the frequency domain. Gordis [27] and He [28] both give frequency domain interpretations of the Error Matrix Method.

Dynamic Reaction Methods localize the finite element model errors by examining the residues of the eigensystem using the experimental mode shapes and frequencies. Large residues indicate errors at the degrees of freedom. The experimental mode shapes are assumed to be known, either by measurement or expansion. Fissette et al. developed the Force Balance Method [29], where a vector of spatially localized unbalanced forces is used to find the degrees of freedom in error. By assuming a form for the model errors, the correction matrices are found.

Many of the finite element model error localization methods presented require either measurement of all degrees of freedom, reduction/condensation of the original system, or expansion of the experimental mode shapes to the size of the original system. In practical applications for space structures, measurement of all degrees of freedom is not possible. As stated previously, reduction/condensation of the original 
system leads to errors and error locations which are suspect [18]. Expansion of mode shapes to the original size of the system has been explored by many researchers, but as with the reduction methods, there is no general correlation between the actual mode shape and the accuracy of the expanded mode shape [30]. The only method which is capable of handling partial information, i.e. a subset of the frequencies and measurement of a subset of the degrees of freedom, is the Sensitivity Method.

Finite element error localization techniques are usually used for updating the physical parameters of the system, assuming that they have deterministic errors. Multiple identification data sets, however, give more information than deterministic errors. The errors are actually stochastic in nature. The mean can be thought of as the modeling error, and can be used in the updating and localization techniques given previously. The variance can be thought of as the repeatability of the experiment, and can be used to develop an uncertainty model for robust control design.

There is little research in the area of repeatability of experiments, especially for the specific use of control design. Collins et al. [31] assume statistical properties for the design parameters, in order to iteratively localize the uncertainties. This is very similar to the Sensitivity Method, except the variances of the design parameters are chosen to reflect the user's confidence in the original estimate. Also, in an interesting series of papers, Hasselman et al. [32-34] group together generically similar structures into three databases, an example of which is Research Models of Large (truss-type) Space Structures. The database can then be used to create a probability model of a space structure, where a mean model can be formed, with a distribution of errors of the modal parameters. Although quite conservative for specific designs, applicability of this database to the initial design phase of space structures is very high.

One of the most thorough examinations of the repeatability of ground and space hardware is the Middeck 0-g Dynamics Experiment (MODE) [35,36]. On the ground and in space, the variation in modal parameters, specifically frequency and damping, were examined as functions of disturbance force amplitude, joint preload, reassembly, shipset (different hardware), and suspension change. Joint preloads in the MODE experiment introduced weak nonlinearities, while the inclusion of a rotary joint intro- 
duced moderate nonlinearities, especially in the torsion mode. Comparing the modal parameters of two sets of hardware designed from the same design plans, the overall trends were the same (i.e. pole-zero patterns), however, there were small uncertainties in frequency from $0-5 \%$. As the frequencies of the suspension system were increased, the test article frequencies increased and damping decreased, especially in the lower modes. The repeatability of the hardware was also tested by using reassembly. The variance during the tests was approximately $1 \%$ in frequency and $1 / 2 \%$ in damping, although this was quite larger for the configuration that included the moderately nonlinear rotary joint.

The work in this thesis builds upon the current error localization literature by introducing an additional matrix method and refining the sensitivity method described previously $[31,23,26]$. Both methods utilize only measured uncertainties, and do not reduce the system or expand the measured mode shapes. The two methods are also extended to include variance uncertainty localization for the development of bounds in an uncertainty model. It will therefore be refered to as uncertainty localization throughout this work. In addition, as a result of the extremely large amount of MACE on-orbit data, a complete examination of the repeatability of a structure in both 1-g and $0-\mathrm{g}$ environments is also given.

\subsection{Thesis Outline}

Chapter 2 gives a preliminary discussion of the models and hardware used throughout this thesis. The Middeck Active Control Experiment (MACE), a shuttle flight experiment that flew on STS-67 in March 1995, is introduced as the motivation and experimental validation of the work in this thesis. A detailed description of both the 1-g and $0-\mathrm{g}$ finite element models is given. Next, a thorough assessment of modeling uncertainties is presented, primarily focusing on uncertainties between the finite element model and hardware.

Chapter 3 details the development, of the $1-\mathrm{g}$, termed Nominal, uncertainty model. First, a testing summary is presented to capture uncertaintics between the model 
and hardware. Next, an identification technique is introduced called the Discrete Extended Kalman Filter (DEKF). This technique satisfies allows the easy parameterization of the finite element model and additional dynamics needed in an identification/parameter estimation method to accurately identify the modal parameters, as well as giving additional information in the form of an error covariance matrix. The development of an uncertainty model based on sets of measurement models and identified parameters from the DEKF is given, which is in the form of mean errors and bounds on critical parameters of the system. Finally, the development of the 1-g uncertainty model for MACE is shown both to verify that the DEKF and uncertainty model can be developed for a practical system, and to support the work in the following chapters.

Chapter 4 develops the methods that take the 1-g and $0-\mathrm{g}$ finite element models, and the 1-g uncertainty model, and predicts the 0-g uncertainty model. The idea proposed is to localize both the mean error and variance uncertainties in the eigenvalues and eigenvectors to specific degrees of freedom, thus forming mass and stiffness uncertainties. Once localized, a mapping between the 1-g and 0-g systems can be made. Therefore the mass and stiffness uncertainties of the $0-\mathrm{g}$ system can be developed. The $0-\mathrm{g}$ uncertainty model can then be predicted by projecting the mass and stiffness uncertainties into $0-\mathrm{g}$ to get mean error and variance uncertainties in the $0-\mathrm{g}$ eigenvalues and eigenvectors. The mean error and variance uncertainties can then be used to form the $0-\mathrm{g}$ uncertainty model, in the form of mean errors and bounds on critical parameters of the system.

Experimental verification of the uncertainty localization and projection methods is given in Chapter 5. Because of the uniqueness of the MACE experiment, there are multiple data sets of two configurations of the test article in both 1-g and $0-\mathrm{g}$. Therefore, two examples are given to validate these techniques using MACE data.

Chapter 6 gives a summary of the usefulness of an uncertainty model for control design. This applies for a general uncertainty model and control design model, not just the $1-\mathrm{g}$ and $0-\mathrm{g}$ cases examined in this thesis. An overview of controller synthesis and analysis methods which benefit from the use of an accurate uncertainty model 
is given. In addition, four cases of how to utilize the mean errors and bounds on critical parameters of the system are presented, each of which are different in their conservatism. Finally, two $0-\mathrm{g}$ closed loop controllers from the MACE experiment are presented. One utilizes the uncertainty model in the most conservative manner, while the other utilizes the uncertainty model in the least conservative manner.

\subsection{Contributions}

The significant contributions of this work are given here in order to understand where they fit with the work presented. In the end, a list of the significant contributions will again be given.

In Chapter 2, the detailed summary of uncertainties between a finite element model and hardware is a contribution. Although parts of this summary have been detailed in other works, this is the most detailed of its kind.

In Chapter 3, the testing process for capturing the finite element model uncertainties in the data is a contribution. This is especially true because it is one of the first set of tests that recognize that hardware is actually stochastic, rather than deterministic. Therefore, one of the more significant contributions of this work is the development of an uncertainty model, which consists of bounds, as well as mean errors, on critical parameters of the system.

Chapter 4 contains the most significant contributions of this work. There are many techniques which have been developed for localization of errors in finite element models. The matrix technique is unique in its development, but is quite limited in its application because it is not physically based. The sensitivity method has been used previously to localize errors in finite element model. This work, however, presents a more structured method than presented previously. In addition, the recognition and localization of both mean error and variance uncertainties is quite unique in its application. Finally, most of the error localization methods work only to improve the finite element model. This work is the first to utilize the methods both for projection into another environment $(0-\mathrm{g})$, and for control design. 
Chapter 5 is unique in its experimental validation of the methods presented. It is one of the more thorough examinations of the modeling and testing of structures in 1-g and 0-g, primarily because of the success of the MACE experiment.

And finally Chapter 6 presents four unique cases of how to utilize both the mean errors and bounds of the uncertainty model for control design using methods developed previously. In addition, experimental validation of the usefulness of the uncertainty model is presented in the form of $0-\mathrm{g}$ controllers implemented on MACE during STS-67. 


\section{Chapter 2}

\section{Discussion of Models and}

\section{Hardware}

In order to obtain better clarity throughout this work, a preliminary discussion of the hardware and finite element models, along with associated uncertainties is given first. The Middeck Active Control Experiment (MACE) is introduced as experimental verification for the techniques presented to assure their practicality. A complete description of the modeling process is given to summarize the modeling work on MACE and to motivate the work in this thesis. The hardware and finite element model are presented in their entirety in order to gain full insight into the uncertainty localization and projection techniques for a practical system. Finally, a thorough assessment of finite element model uncertainties is presented which aids in the development of the uncertainty localization techniques and gives insight into which uncertainties can be easily localized. In addition, by thoroughly examining modeling uncertainties, a set of experimental tests can be developed which aid in the observation of each of the different types of uncertainties. 


\subsection{Middeck Active Control Experiment (MACE)}

The Middeck Active Control Experiment (MACE), shown in its 1-g configuration in Figure 2.1, is a shuttle middeck experiment that flew on the Space Shuttle Endeavour in March 1995 (STS-67). MACE was designed to investigate the various modeling and control design issues associated with the change in operational environment of a flexible spacecraft from $1-\mathrm{g}$ to $0-\mathrm{g}$ [37]. The primary objective of the MACE program is develop a well verified set of design techniques that allow designers to have confidence in the eventual orbital performance of future spacecraft that cannot be dynamically tested on the ground in a sufficiently realistic $0-\mathrm{g}$ simulation. In order to achieve this objective, a complex mesh of analytical modeling, ground testing, and onorbit redesign was implemented which aided in the development of tools to confidently and accurately predict open loop and closed loop behavior without the benefit of 0-g data (i.e. prior to launch). MACE builds upon the concepts of the Middeck 0-Gravity Dynamics Experiment (MODE), which explored the characterization of fluid dynamics, space station structure, and crew motion dynamics in 0-g on STS-40, STS-48, and STS-62 $[35,38]$.

The specific program objectives were as follows:

- Assess impact of gravity perturbations and associated uncertainties on the effectiveness and predictability of multivariable robust control.

- Develop a set of Controlled Structures Technology (CST) procedures for model development, controller synthesis and closed loop evaluation.

- Demonstrate robust control of structural flexibility in the micro-gravity environment.

- Augment preprogrammed control with on-orbit system identification and control redesign.

- Evaluate modeling and control on a test article representative of a particular mission architecture.

- Develop a reusable dynamics and control laboratory for the shuttle middeck. 


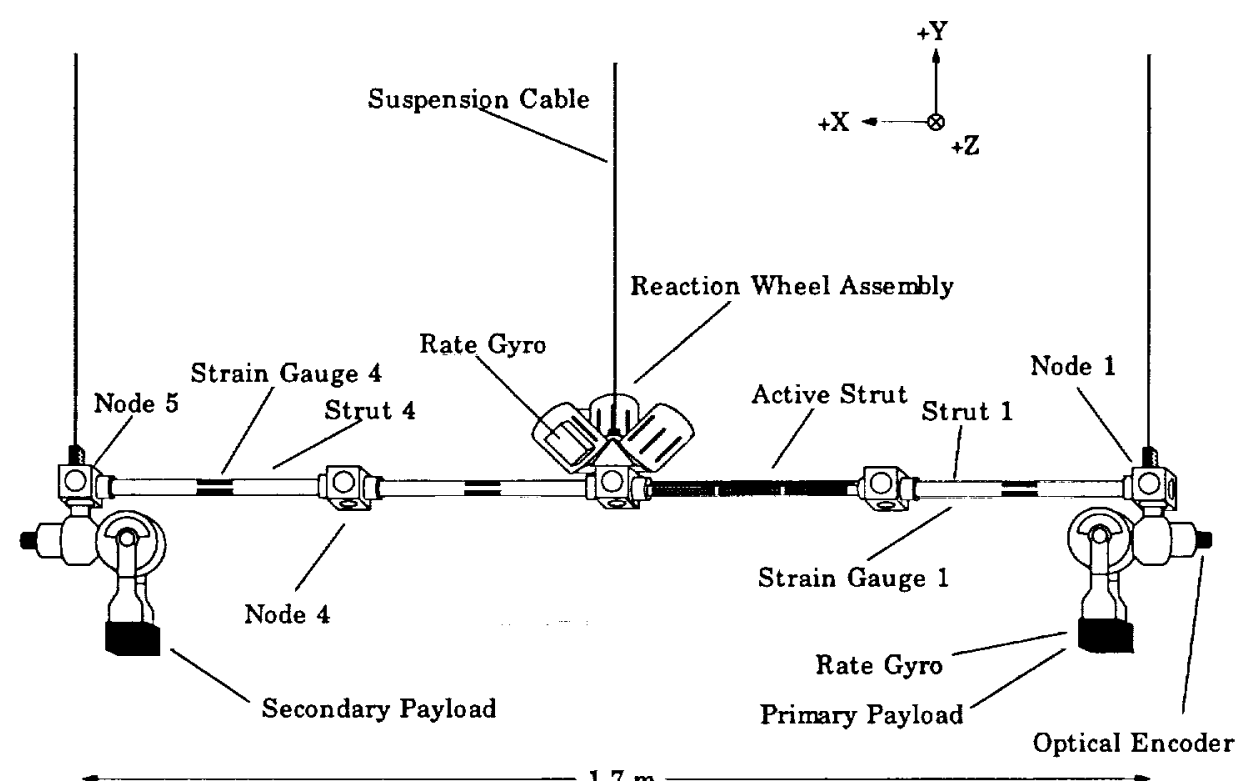

Figure 2.1: MACE Flight Model Test Article

\subsubsection{Modeling Process}

The primary motivation for the work in this thesis came from challenges developed during the MACE program. In order to motivate and clearly understand this work, a detailed description of the modeling process for MACE is given. Note that this is a specific example of the general process defined in Chapter 1 .

In order to satisfy the specific program objectives listed above, the MACE program adopted a complex blend of analytical modeling, ground testing, and on-orbit redesign procedures. Figure 2.2 shows how the MACE program has been designed to investigate each of these techniques.

A 1-g analytical finite element model (FEM) is developed, and updated using modal identification (step A) [39] and measured models (step C) [40]. The performance of controllers derived from the FEM is used to identify inadequacies in the model providing information for further model updating (step B) [41]. The performance of controllers derived from measurement models is used to determine when 


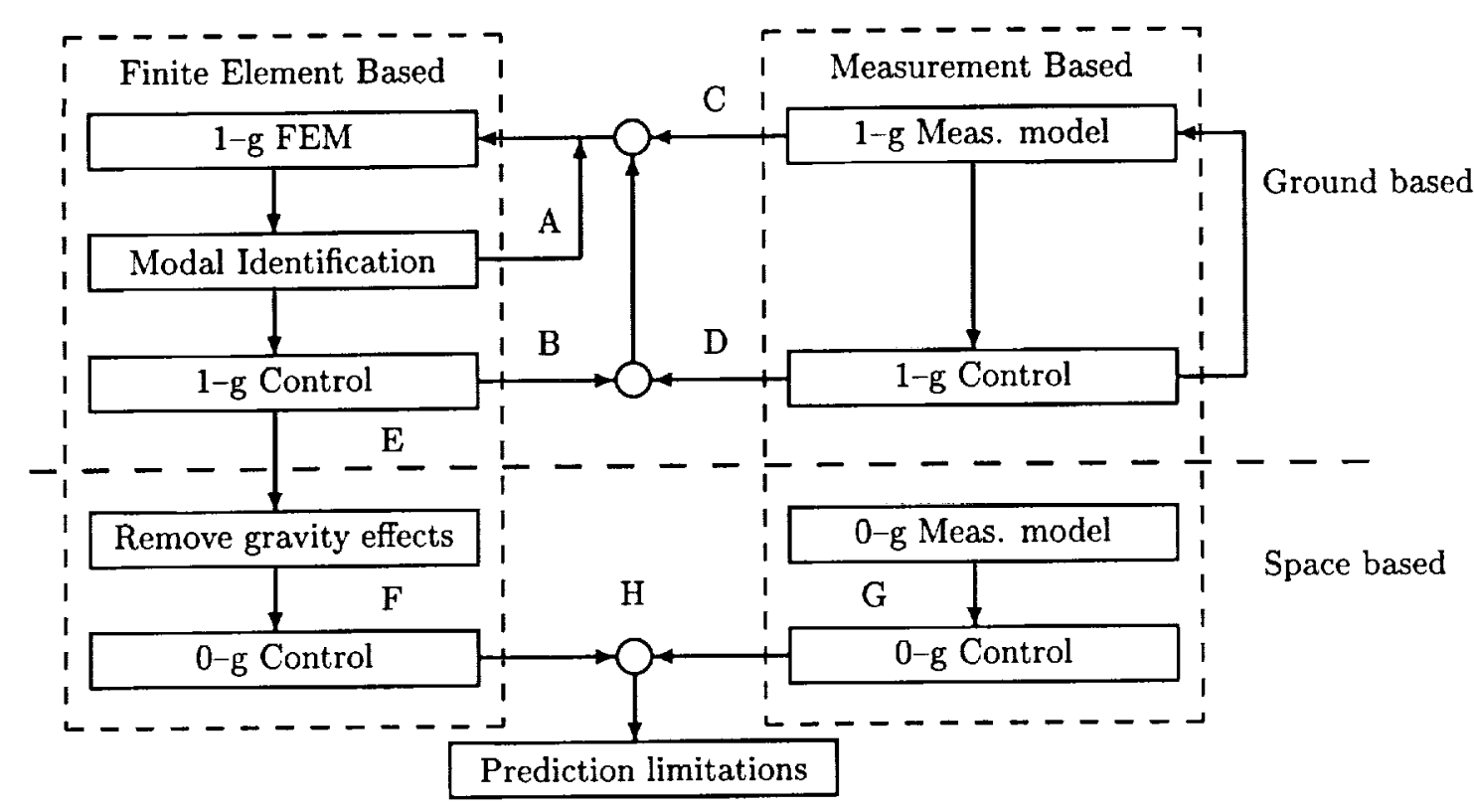

Figure 2.2: Modeling and Control approach for MACE

FEM refinement is complete (step D). The $1-\mathrm{g}$ finite element model is essentially updated when the closed loop performance for both measurement and finite element based controllers are equivalent (steps B and D in Figure 2.2) [42].

In order to develop controllers prior to flight, the FEM must be used to predict the $0-\mathrm{g}$ dynamics because it is the only model that is physically based. The gravity and suspension parameters are removed from the updated 1-g FEM to provide a $0-\mathrm{g}$ model (step E). The 0-g FEM is then to be used for design of flight controllers (step $F$ ). In addition, an on-orbit system identification is performed to provide a $0-\mathrm{g}$ measurement based model, which is then be used to design further controllers (step G). Finally, FEM and measurement based controllers are compared to judge the cost/benefit of on-orbit redesign of controllers (step H). Summaries of the on-orbit modeling and control results are given in Refs. 43-45.

The stability and performance of the controllers designed for the ground-based tests were typically analyzed using open-loop data. As discussed in Ref. 46, this reduces the control design iteration time because it accurately captures the discrepancies between the model and the test article. However, prior to flight, no data exists for analyzing the controllers designed in Step F of Figure 2.2. Thus a new approach 
had to be developed that extends the insights gained from the ground tests and provides a quantitative measure of the possible errors in the 0 -g control design model that can be used to analyze the compensators designed prior to flight. This approach is the development of the $0-\mathrm{g}$ uncertainty model.

A few of the insights found during the development of the $0-\mathrm{g}$ uncertainty model are given here. First, extraction of all modal parameters, specifically the frequencies, damping ratios, and mode shapes, is quite difficult to do if using a general measurement model. Prior to and during the flight, a technique called Frequency domain Observability Range Space Extraction (FORSE) [40] was used to create an initial measurement model, and a Nonlinear Logarithmic Least Squares Method (NLLS) [47] was used to obtain an even better fit to the data. While these techniques created a very accurate fit to the data, estimating the frequency and mode shape information from this model became difficult because of repeated modes, estimating normal modes from measured complex modes, and the inherent coupling between the structural system and additional dynamics such as time delays, sensor dynamics, and the closed loop servo system. It was for these reasons that only 1-g frequency uncertainties were used in developing the $0-\mathrm{g}$ uncertainty model for flight. A complete summary of the uncertainty model used for flight is given in Ref. 48 .

It became clear, however, that for the projection technique to be accurate, more than the 25 frequency uncertainties were needed. Measured mode shapes from all of the sensors would provide an additional 400 pieces of information which could be used in the procedure, thus making it far more accurate. The work in this thesis is a more complete analysis of the development of 0-g uncertainty model given in Ref. 48 . It draws upon the experiences and data from both ground and space based testing of MACE.

Another piece of modeling insight developed during the MACE program was that of the best measurement model for control design. During the flight, the finite element model based controllers essentially performed better than the redesigned controllers based on the more accurate measurement model [44]. The source of this nonintuitive result may lie within the structure itself. When three identifications were performed 
at different disturbance amplitude levels, the damping in a few modes was strikingly different, implying a strong amplitude dependent nonlinearity. The measurement

model reproduced the data so accurately that the nonlinearity may have been fit also, such that it detrimentally affected the closed loop controllers. For more detailed explanation of the on-orbit results, refer to Refs. 43-45

A summary of the primary modeling insights developed during the MACE program include:

- Development of a 0-g finite element model accurate enough for robust control design can be accomplished using ground based modeling and testing, and removal and gravity and suspension effects.

- Predictable closed loop results for controllers designed prior to flight requires both an accurate 0 -g finite element model and an accurate $0-\mathrm{g}$ uncertainty model.

- Prediction of an accurate 0-g uncertainty model requires measurement of frequency and well as mode shape information.

- Extraction of modal parameters from measurement models is quite difficult when measurement models are not physically based.

- The best measurement model for control design is both accurate and physically based.

\subsubsection{Hardware Description}

The MACE Flight Model (FM) test article, illustrated in Figure 2.1 consists of 4 straight polycarbonate tubes assembled into a bus, each separated by aluminum collars. One strut is actually square hollow, with piezoelectrics mounted on each side to provide bending strain. Two two-axis pointing/scanning payloads are attached at each end, each with gimbals actuating about the $\mathrm{X}$ and $\mathrm{Z}$ axes via $\mathrm{DC}$ torque motors. One is primarily used as a disturbance source (secondary payload), while the other creates the pointing performance metric (primary payload). A reaction wheel assem-

bly at the center node, comprised of three orthogonally mounted DC servo motors with an inertia wheel mounted on each, is used to provide attitude torques in three axes. 
The MACE FM also comprises a variety of sensors for identification and closed loop control. Each gimbal contains laser rotary encoders measuring the relative angular rate of each axis (4 total). A two-axis rate gyro platform measuring the inertial rate about the $X$ and $Z$ axes is attached inside of the primary payload to provide a measure of the pointing performance metric. Three single axis rate gyros are also mounted on the side of the reaction wheel cans, providing collocated inertial rate measurements in three axes. Each reaction wheel also has a tachometer measuring the relative speed of each wheel. Each strut is also instrumented with two strain gauge pairs at its midpoint to provide a measure of the bending strains about the $\mathrm{Y}$ and $\mathrm{Z}$ axes.

The Engineering Support Module (ESM) (not shown) houses many of the additional pieces of equipment which support the test article. These include: the digital control computer, two channel signal generation, memory storage, disk drive, power conditioning for the actuators, and signal conditioning for the sensors. The real time control computer operates at a $500 \mathrm{~Hz}$ sampling rate, and can handle compensators of up to 80 states. The ESM is attached to the MACE FM test article through a power and data umbilical. In 1-g, the test article is supported by a three point active pneumatic/electric low frequency suspension system. Also not shown is a $\mathrm{Ku}$-Band Interface System (KIS) which provided 40 Megabyte uplink and downlink capability between Mission Control and the Space Shuttle during STS-67. A detailed description of the MACE hardware is given in Refs. 49,37

\subsubsection{STS-67}

The MACE experiment flew on the middeck of the United States Space Shuttle Endeavour during the STS-67 mission on March 2-18, 1995. The primary payload of the mission was Astro-2, the second dedicated Spacelab mission to conduct astronomical observations in the ultraviolet spectral regions. The Commander and Pilot of STS-67, Stephen S. Oswald and William G. Gregory, were in charge of on-orbit operations for MACE. MACE was originally scheduled for 3 days of on-orbit operations with the 
following timetable:

Day 1: -Identification of test article and downlink of data -Implementation of $0-\mathrm{g}$ controllers designed prior to flight -Creation of $0-\mathrm{g}$ measurement model (on ground)

Day 2: -Implementation of pre-programmed controllers designed prior to flight -Redesign of $0-\mathrm{g}$ controllers based on measurement model (on ground)

Day 3: -Uplink of redesigned 0-g controllers -Implementation of redesigned $0-\mathrm{g}$ controllers

As a result of STS-67 being extended to a 16 day mission and the unwavering support of the crew, the MACE team received much more than the originally planned schedule. MACE operations during the mission included: 14 days of testing in three different configurations; over 550 protocols (algorithms), both pre-programmed and redesigned, were implemented for system identification, CST control, and command input shaping; there were 13 downlinks of data totaling over 500 Megabytes, and 4 uplinks of redesigned protocols; 5.4 Gigabytes of data was collected; and audio and video downlink of the crew's observations were used to facilitate redesign.

A breakdown of the CST control protocols that were implemented on-orbit is given in Table 2.1, where FEM refers to finite element based controllers, and MM refers to measurement model based controllers. Notice that more than $50 \%$ of the controllers implemented on-orbit were designed prior to flight using the finite element model and projected uncertainties (i.e. using no flight data). This shows the weighted importance for the MACE program of the developing accurate control design and uncertainty models for accurately predicting closed loop results prior to flight.

\begin{tabular}{l|c|c|l} 
Type & Model & Number & Evaluation \\
\hline Pre-programmed & FEM & 204 & Projected Uncertainties \\
Redesign & FEM & 44 & 0-g Data \\
Redesign & MM & 132 & 0-g Data
\end{tabular}

Table 2.1: Breakdown of control protocols implemented on STS-67. 


\subsubsection{Finite Element Model}

As stated previously, the topics of finite element modeling and updating are quite large and complex, and are not the focus of this work. These models are therefore assumed to be complete at the beginning of this work. The $1-\mathrm{g}$ and $0-\mathrm{g}$ finite element models are due to Glaese and Miller $[50,39]$. A summary of the models is given here to aid in the discussions of the uncertainty localization and projection techniques presented in the subsequent chapters.

In order to fully understand which degrees of freedom the uncertainties are localized to in the finite element model, a breakdown of the nodal numbering system is presented. Table 2.2 gives a summary of the nodes and elements for the model. Figure 2.3 shows a stick figure of the MACE FM hardware along with the nodal numbering scheme. Note that the nodal numbering of the model is structured such that the $0-\mathrm{g}$ model is developed by deleting nodes $81-113$ from the 1-g model. For a more complete description of the finite element models, please refer to Refs. 50, 39 .

In addition to the physical modeling of the structure, gravity effects are also incorporated into the 1-g model. These gravity effects include sag and twist predeformations, and pre-loading of members, pendular effects on articulating appendages, and suspension cable violin behavior. These effects are included by adjusting the 1-g

\begin{tabular}{|l|c|l|l|}
\hline Type & Element & 1-g Node Number & 0 -g Node Number \\
\hline \hline Aluminum Collar & Beam & $\begin{array}{l}1-3,12-14,27-29, \\
38-40,49-51\end{array}$ & $\begin{array}{l}1-3,12-14,27-29, \\
38-40,49-51\end{array}$ \\
\hline Lexan Strut & Beam & $4-11,30-37,41-48$ & $4-11,30-37,41-48$ \\
\hline Active Strut & Beam & $15-26$ & $15-26$ \\
\hline Reaction Wheel Rate Gyros & Beam & $52-54$ & $52-54$ \\
\hline Primary Gimbal & Beam & $55-62$ & $55-62$ \\
\hline Secondary Gimbal & Beam & $63-70$ & $63-70$ \\
\hline Reaction Wheel Assembly & Beam & $71-80$ & $71-80$ \\
\hline Suspension System & Beam & $81-113$ & n/a \\
\hline
\end{tabular}

Table 2.2: Nodal numbering and element descriptions for the MACE FM finite element model. 


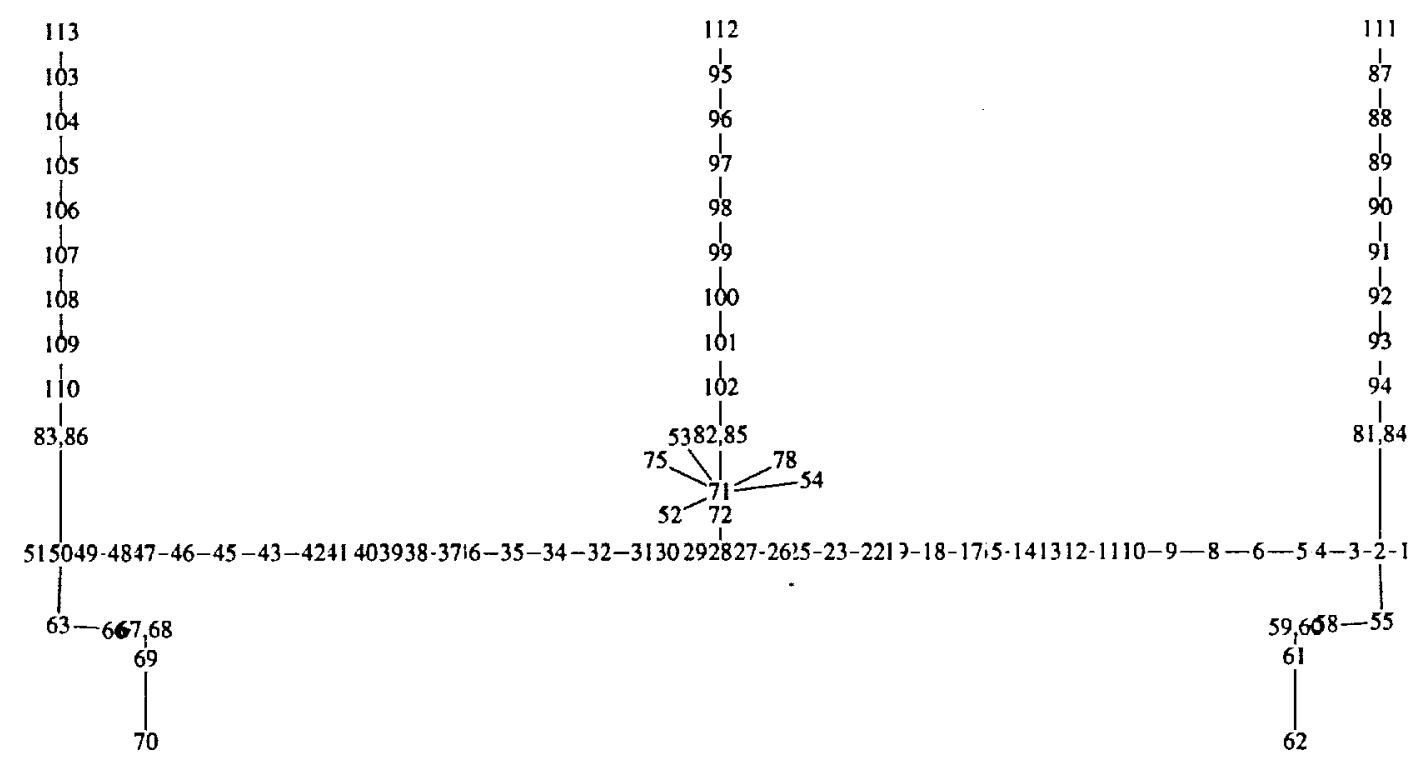

Figure 2.3: MACE FM nodal numbering in the 1-g finite element model.

stiffness matrix using a iterative, nonlinear static, stress stiffening procedure.

Table 2.3 shows the frequencies and proportional damping ratios for the controllable/observable modes in the 1-g and $0-\mathrm{g}$ finite element models for the MACE FM. The damping in the MACE FM was assumed to be both proportional and concentrated, however. The proportional damping was identified using accurate measurement models fit to transfer function data. The concentrated damping is the result of point dampers in each of the gimbals and reaction wheels, and at the end of each of the suspension cables. In developing an input-output model for MACE, additional dynamics and gains were added. These included sensor dynamics and notch filters on the rate gyros, Bessel filters on the strain gauges and tachometers, digital computer delays, and various conditioning gains on each of the actuator and sensor channels.

In addition to the sensor, actuator, and computer modeling, the nominal system for the MACE test article also includes two servo systems. Three speed servos from 


\begin{tabular}{|c|c|c|c|c|}
\hline \multirow[b]{2}{*}{ Mode Type } & \multicolumn{2}{|c|}{$1-\mathrm{g}$} & \multicolumn{2}{|c|}{$0-\mathrm{g}$} \\
\hline & $f(\mathrm{~Hz})$ & $\zeta$ & $f(\mathrm{~Hz})$ & $\zeta$ \\
\hline Torque wheel \#1 & 0.00 & 1.000 & 0.00 & 1.000 \\
\hline Torque wheel \#2 & 0.00 & 1.000 & 0.00 & 1.000 \\
\hline Torque wheel \#3 & 0.00 & 1.000 & 0.00 & 1.000 \\
\hline Suspension - bounce & 0.20 & 0.100 & $\mathrm{n} / \mathrm{a}$ & $\mathrm{n} / \mathrm{a}$ \\
\hline Suspension - rotary pendulum & 0.24 & 0.091 & $\mathrm{n} / \mathrm{a}$ & $\mathrm{n} / \mathrm{a}$ \\
\hline Suspension - tilt & 0.44 & 0.034 & $\mathrm{n} / \mathrm{a}$ & $\mathrm{n} / \mathrm{a}$ \\
\hline Primary X Gimbal & 1.19 & 0.038 & 0.00 & 0.00 \\
\hline Primary Z Gimbal & 1.23 & 0.055 & 0.00 & 0.00 \\
\hline Secondary X Gimbal & 1.26 & 0.050 & 0.00 & 0.00 \\
\hline Secondary Z Gimbal & 1.30 & 0.060 & 0.00 & 0.00 \\
\hline Suspension - compound pendulum & $1: 84$ & 0.066 & $\mathrm{n} / \mathrm{a}$ & $\mathrm{n} / \mathrm{a}$ \\
\hline $1^{\text {st }} \mathrm{Z}$ bending & 2.24 & 0.022 & 2.27 & 0.021 \\
\hline $1^{\text {st }} Y$ bending & 4.60 & 0.070 & 3.73 & 0.064 \\
\hline Suspension $-1^{\text {st }} \mathrm{X}$ violin & 7.13 & 0.014 & $\mathrm{n} / \mathrm{a}$ & $\mathrm{n} / \mathrm{a}$ \\
\hline Suspension $-1^{\text {st }} \mathrm{Z}$ violin & 7.24 & 0.006 & $\mathrm{n} / \mathrm{a}$ & $\mathrm{n} / \mathrm{a}$ \\
\hline $2^{\text {nd }} Z$ bending & 9.56 & 0.012 & 9.53 & 0.041 \\
\hline $2^{\text {nd }} Y$ bending & 9.80 & 0.039 & 9.47 & 0.029 \\
\hline $3^{\text {rd }} \mathrm{Z}$ bending & 11.04 & 0.037 & 10.95 & 0.042 \\
\hline $3^{\text {rd }} \mathrm{Y}$ bending & 11.29 & 0.059 & 11.13 & 0.056 \\
\hline Suspension $-2^{\text {nd }} \mathrm{Z}$ violin & 11.66 & 0.013 & $\mathrm{n} / \mathrm{a}$ & $\mathrm{n} / \mathrm{a}$ \\
\hline $3^{\text {rd }} \mathrm{Y}$ bending & 13.14 & 0.054 & 13.44 & 0.067 \\
\hline Suspension $-3^{\text {rd }} \mathrm{Z}$ violin & 14.68 & 0.020 & $\mathrm{n} / \mathrm{a}$ & $\mathrm{n} / \mathrm{a}$ \\
\hline Suspension $-2^{\text {nd }} \mathrm{X}$ violin & 15.12 & 0.021 & $\mathrm{n} / \mathrm{a}$ & $\mathrm{n} / \mathrm{a}$ \\
\hline $4^{\text {th }} \mathrm{Z}$ bending & 16.19 & 0.018 & 16.11 & 0.018 \\
\hline $4^{\text {th }} \mathrm{Y}$ bending & 17.82 & 0.009 & 17.85 & 0.009 \\
\hline $5^{\text {th }} \mathrm{Y}$ bending & 24.90 & 0.014 & 25.03 & 0.014 \\
\hline $5^{\text {th }} \mathrm{Z}$ bending & 39.41 & 0.014 & 39.56 & 0.014 \\
\hline $6^{\text {th }} \mathrm{Z}$ bending & 48.84 & 0.019 & 49.18 & 0.019 \\
\hline $6^{\text {th }} \mathrm{Y}$ bending & 49.01 & 0.016 & 49.67 & 0.017 \\
\hline $7^{\text {th }} \mathrm{Y}$ bending & .56 .16 & 0.015 & 56.97 & 0.016 \\
\hline
\end{tabular}

Table 2.3: Frequency, damping ratio, and description of each mode retained in the finite element model for the MACE FM test article in 1-g and $0-\mathrm{g}$. (0-60 $\mathrm{Hz}$ range) 
the collocated tachometers to each of the reaction wheels and a DC spin are used to prevent nonlinear stiction in the wheels from affecting the open and closed loop experiments. There are also four proportional-integral servos from each encoder to collocated gimbal. These are used to act as a nominal pointing system, to reduce the nonlinear stiction effects in the gimbals, and to enable the identification of the system in $0-\mathrm{g}$, where the gimbal modes would otherwise be rigid body modes. Note that the frequencies and damping ratios shown in Table 2.3, especially the gimbal rigid body modes, are substantially different when the gimbal servo systems are included in the model. After retaining only those modes which are controllable/observable in the input-output model, and adding the additional dynamics and damping, the final input-output model has 256 states, 23 outputs, and 9 inputs.

\subsection{Assessment of Modeling Uncertainties}

The most difficult aspect of this thesis is localizing the uncertainties, both mean error and variance, to specific degrees of freedom. If this is accurately accomplished, the projection techniques such as $1-\mathrm{g}$ to $0-\mathrm{g}$ can be accomplished in a straightforward manner. The first step in this process is to define all the possible modeling uncertainties, and how they manifest themselves into the model. (Note that this may be difficult in some cases.) A complete description of all uncertainties also provides the theoretical groundwork for the uncertainty localization process. All uncertainties can be divided into the general groups of Linear and Nonlinear. Within these general

groups, 5 sub-groups can be defined which describe the types of uncertainties between the hardware and structural model of a general system. These will be called: Physical, Modeling, Coupling, Testing, and Nonlinearities.

\subsubsection{Physical Uncertainties}

Physical uncertainties are those where the physics of the system has been captured, but the parameters have incorrect values. These uncertainties, which are traditionally treated with updating techniques, can be divided into two smaller groups: Material 


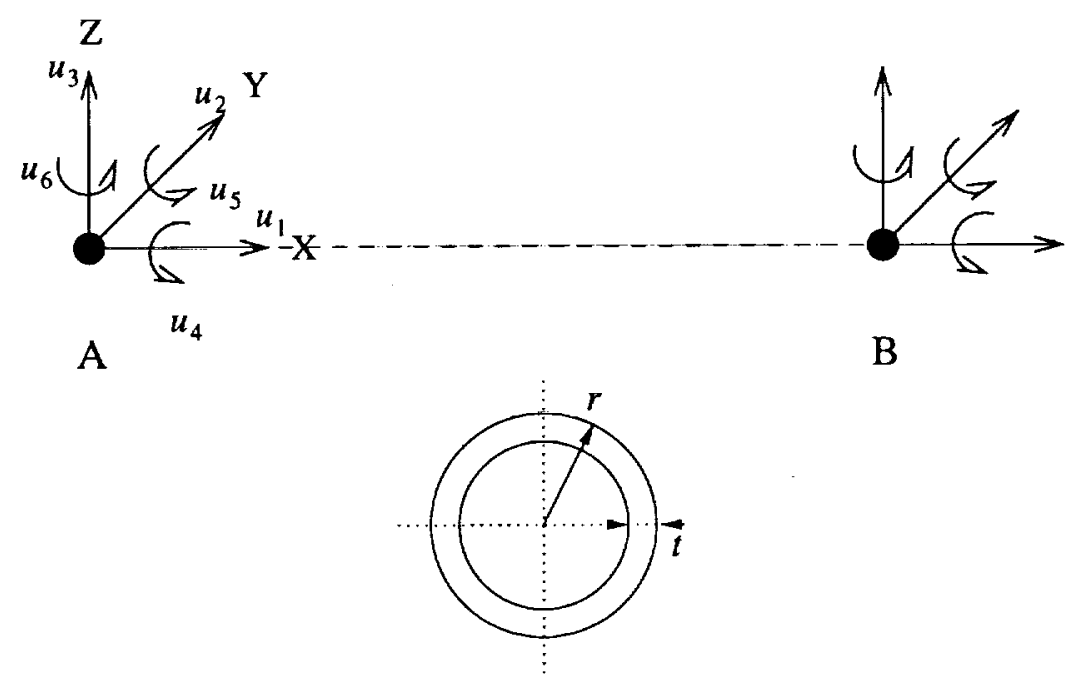

Figure 2.4: Sample beam element and reference axes and notation.

uncertainties such as the modulus of elasticity, density, and Poission's ratio; and Geometric uncertainties such as area and inertia (or their primitives such as radius, width, etc.), and length. These are also the easiest to be updated using uncertainty localization techniques.

To demonstrate the physical uncertainties which may lie inside a specific finite element, a beam element is broken down into its simplest form. Figure 2.4 shows the reference axes and notation used for this beam element. The element has 12 degrees of freedom, being 3 displacements and 3 slopes at each end of the element, or

$$
u=\left[\begin{array}{llllll}
u_{1}^{A} & \cdots & u_{6}^{A} & u_{1}^{B} & \cdots & u_{6}^{B}
\end{array}\right]^{T}
$$

The stiffness and mass matrices for this element can be written in block form

$$
\begin{aligned}
& k=\left[\begin{array}{ll}
k_{\mathrm{AA}} & k_{\mathrm{AB}} \\
k_{\mathrm{AB}}^{T} & k_{\mathrm{BB}}
\end{array}\right] \\
& m=\left[\begin{array}{ll}
m_{\mathrm{AA}} & m_{\mathrm{AB}} \\
m_{\mathrm{AB}}^{T} & m_{\mathrm{BB}}
\end{array}\right]
\end{aligned}
$$

In order to simplify the examination of the possible uncertainties within these elemental matrices, only the sub-matrices $k_{\mathrm{AA}}$ and $m_{\mathrm{AA}}$ are examined, with the other 
sub-matrices matrices understood to be simple extensions. Assuming a tubular, symmetric cross-section, the $6 \times 6$ sub-matrices are given by

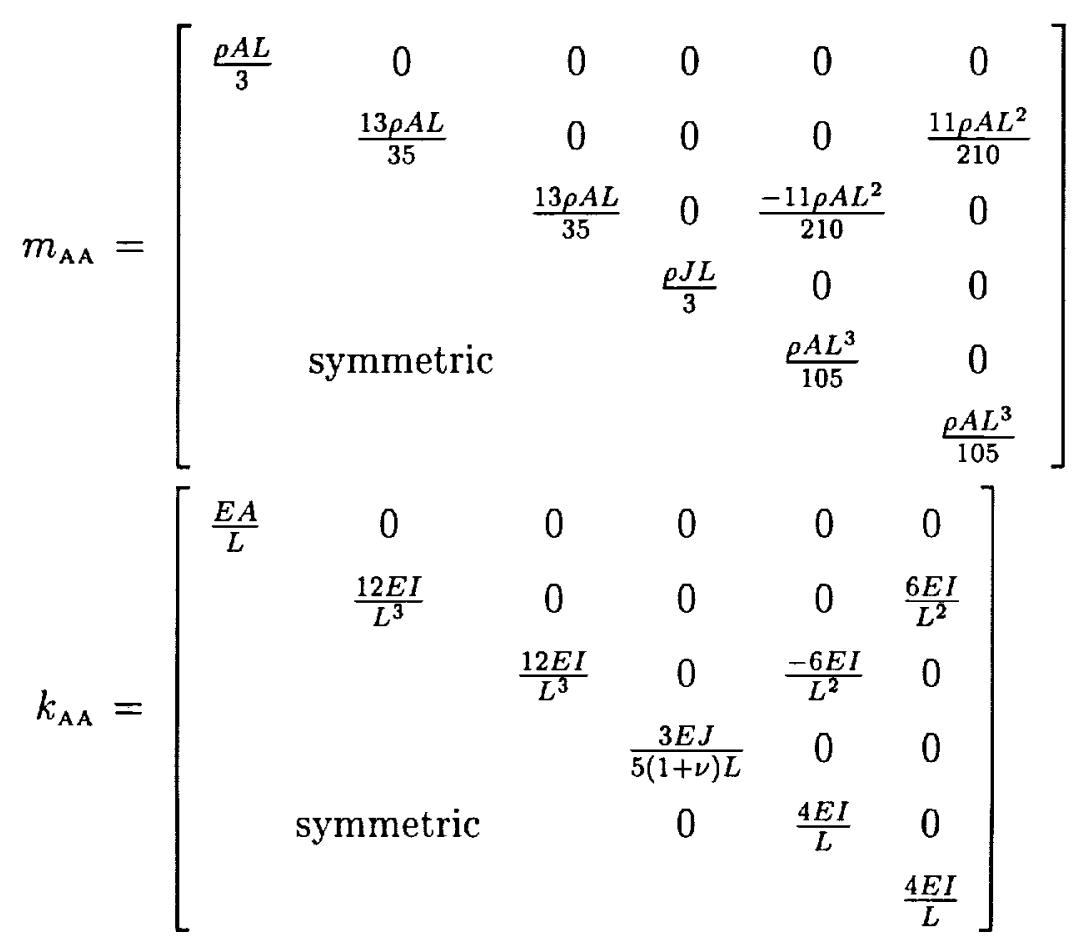

where the area and inertias for a tube can be further written as

$$
\begin{aligned}
I & =\pi r^{3} t \\
J & =2 \pi r^{3} t \\
A & =2 \pi r t
\end{aligned}
$$

where $r$ is the radius and $t$ is the thickness.

Table 2.4 shows a summary of the mass and stiffness change matrices (in \% change of entry), for changes in the material and geometric properties. There are a few items to notice. First, the change in modulus of elasticity $(E)$ and change in density $(\rho)$ only affect the stiffness and mass matrices respectively. Also, a change in the thickness $(t)$ is the same as a change in the modulus of elasticity $(E)$ and density $(\rho)$. As a result, the uncertainty localization techniques and finite element model updating techniques cannot distinguish between these two. The change in radius $(r)$ is similar to the change in the thickness $(t)$ (and combination of changes in modulus of elasticity $(E)$ and density $(\rho)$ ), except for the extensional and torsional degrees of freedom. As with 


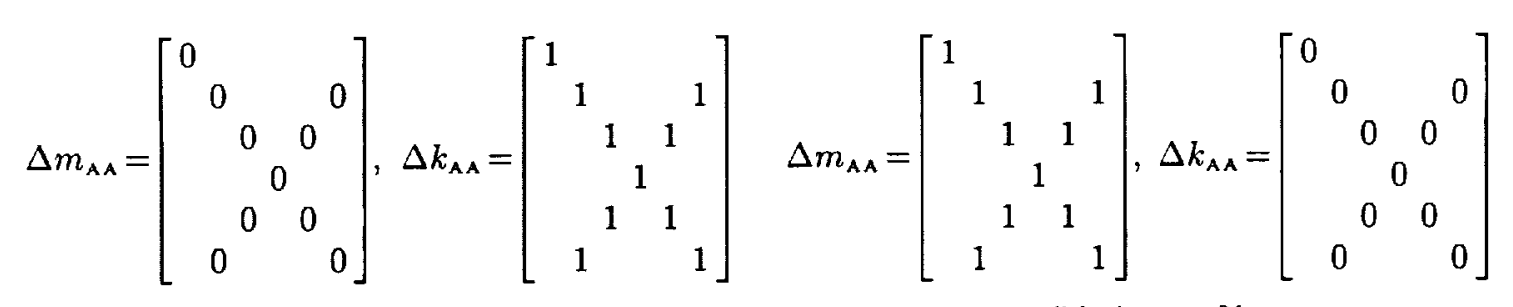

(a) $\Delta E=1 \%$

(b) $\Delta \rho=1 \%$

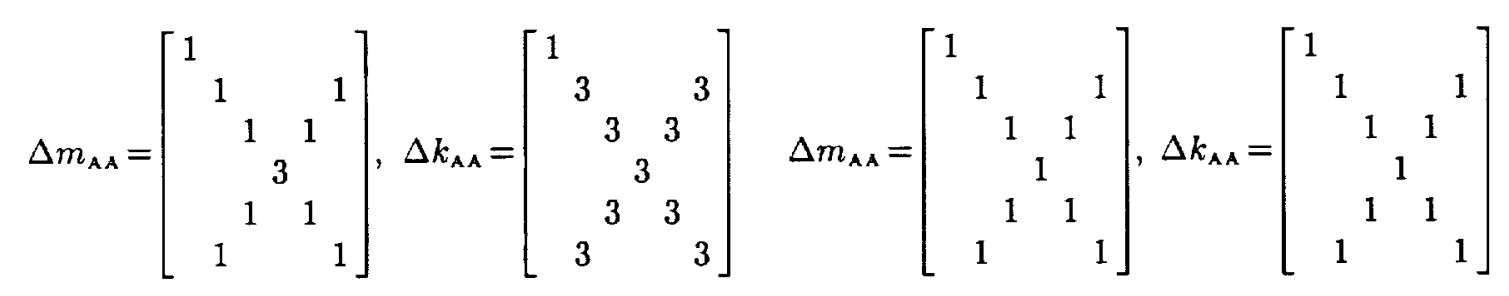

(c) $\Delta r=1 \%$

(d) $\Delta t=1 \%$

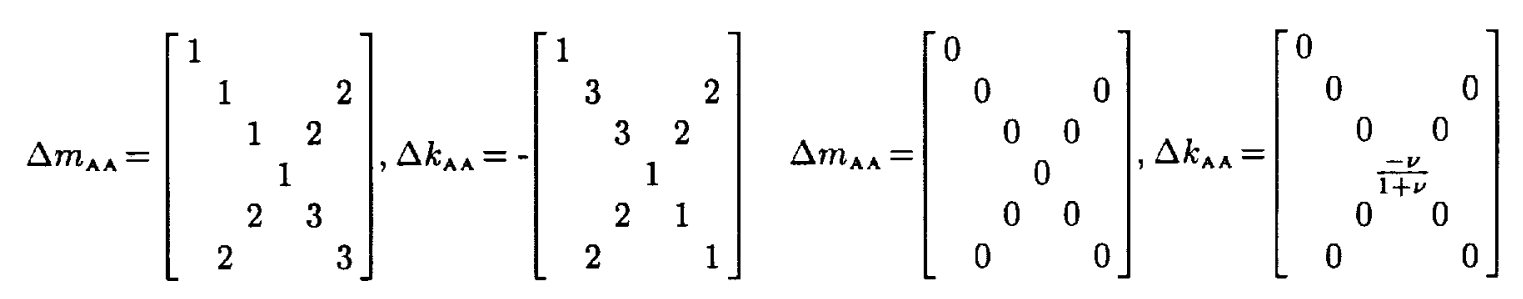

(e) $\Delta L=1 \%$

(f) $\Delta \nu=1 \%$

Table 2.4: Breakdown of mass and stiffness element change matrices for changes in physical parameters for a tubular beam element.

the case of change in the thickness $(t)$, if these degrees of freedom are not measured, it is quite difficult to distinguish the difference between them. The change in length $(L)$ is quite independent from the others, and affects both the mass and stiffness matrices. And the change in Poisson's ratio $(\nu)$ affects only the torsional degree of freedom in the stiffness matrix. If this degree of freedom is not measured, the effects of this change are minimal. Insights such as the relative importance of these change matrices and which of them can be observed are a very important basis for the uncertainty localization techniques. Note that similar analyses can be made for any cross-section and any type of finite element.

\subsubsection{Modeling Uncertainties}

Modeling uncertainties are those errors in which the physics of the hardware has not been represented correctly. These can include discretization issues, selection of 
correct element types, or unmodeled boundary conditions. Most of the uncertainties in this group can be avoided with thorough and correct modeling of the given system.

Discretization errors can usually be avoided by using a fine mesh of elements in the model. If further mesh refinement does not reveal differences in the frequency range of interest, there is usually no discretization errors. In a few cases, such as when a piezoceramic is attached to the top of a more flexible member, a discontinuity can occur in the strain, such that an extremely fine mesh is needed. Many times, however, these problems can be avoided by additional corrections. An example of this is incorporating a static gain into the input-output model of the system in order to overcome inaccurate modeling of the discontinuous strain at the edge of a piezoceramic [51].

The choices of element types are very important in representing the dynamics of the structure. If an incorrect element type is used, such as using a beam element when a shell element is more appropriate, or the boundary conditions are mismodeled, the physics of the model has not been captured correctly. Therefore, any localized adjustment is not physically correct, and cannot be used as a basis for updating or projection.

\subsubsection{Coupling Uncertainties}

There are many different types of systems that couple with the dynamics of the finite element model, only a few of which are explained here. Note that physics of the couplings are assumed to be modeled correctly, but the parameters of which may need adjustment. The couplings that are described in this work are divided into two groups: Internal and External Couplings. Internal Couplings are those additional components of the system which need modeling, but are not necessarily modeled with finite elements. These include boundary conditions, joints and attachments, actuator and sensor dynamics, time delays, various gains, etc. These components must be modeled and then appended to the finite element model. By doing this, these models can be parameterized in the identification technique in order to develop uncertainties attributed to components themselves. Therefore, if they are parameterized in the 
model, the uncertainty localization can be accomplished, even though they have not been included in the finite element model.

External Couplings are those which are applied externally, or due external systems. Three very important External Couplings are aerodynamic, gravity, and control systems. The modeling of the coupling between an aerodynamic model and a finite element model is a very difficult process to undertake [52]. In addition to uncertainties in the finite element model, even larger uncertainties exist in the homogeneous disturbance and aerodynamic effects. Localization for this type of system would be a great challenge.

The coupling of a control system with a model may or may not have a large impact on the uncertainty localization procedure. The closed loop system can usually be parameterized in the identification process, which makes it similar to that of adding actuator or sensor dynamics. However, care must be taken in understanding the impacts of the control system on the other uncertainties. For instance, a high bandwidth compensator may control the closed loop system so well that the sensor signals are much smaller than they would be in open loop. This increases the susceptibility of the system to uncertainties such as sensor noise and nonlinearities. For MACE, the servo systems around each gimbal and reaction wheel actually benefit the uncertainty localization process by reducing the nonlinear stiction effects in the gimbals and reaction wheels. The control system, however, must be parameterized in the identification process. An even more complicated system is one which couples both aerodynamic and control system effects with the finite element model [53].

Wada [54] states that the modeling of the gravity (and suspension) effects represent a major obstacle to the testing of space structures, rather than a minor inconvenience. The coupling of gravity into a system has many different effects such as geometric stiffening of members, initial deformation, and effects on actuators and sensors. Because the focus of this work is on projection of uncertainties from 1-g into $0-\mathrm{g}$, a more detailed explanation of gravity coupling is given here. This summary is due to Rey $[55,56]$, where an excellent overview of the previous work and thorough examination of the modeling of these gravity effects is presented. 


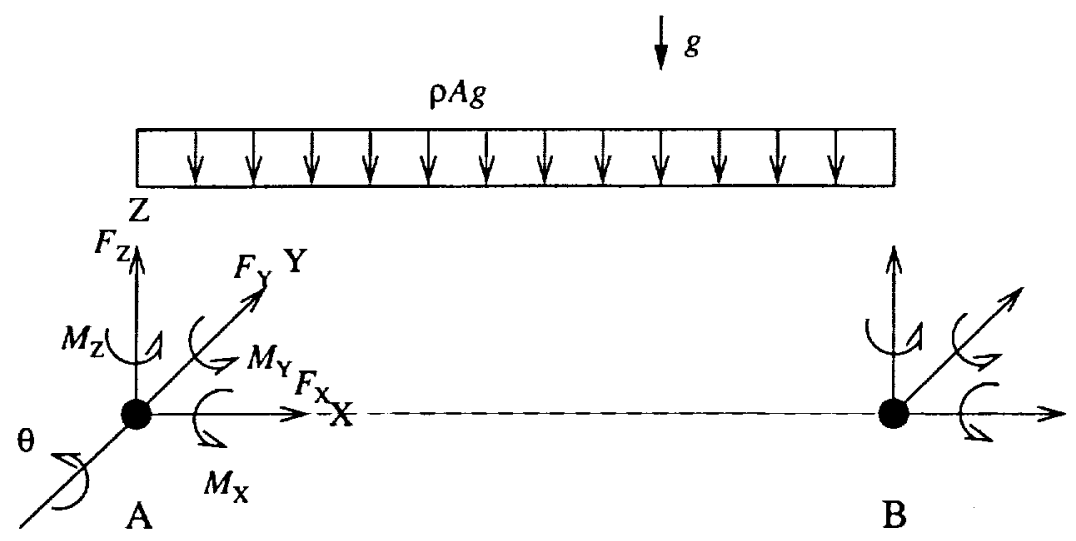

Figure 2.5: Nodal and distributed loads on a beam element in gravity.

The development of an adjustment to the elemental stiffness matrix due to gravity requires stress evaluation and integration of the nonlinear strain-displacement relations. Finite element modeling software usually does this using an iterative method to create the internal stress description. Rey [56] developed an elemental stiffness adjustment due to gravity stiffening by assuming nodal and distributed loads. Figure 2.5 shows a beam element with a distributed gravitational load acting over the element, and nodal forces and moments acting at each end. The nodal forces and moments are due to nodal masses and inertias coupling with gravity in the element. The adjustment to the 1-6 portion of stiffness matrix can then be written as

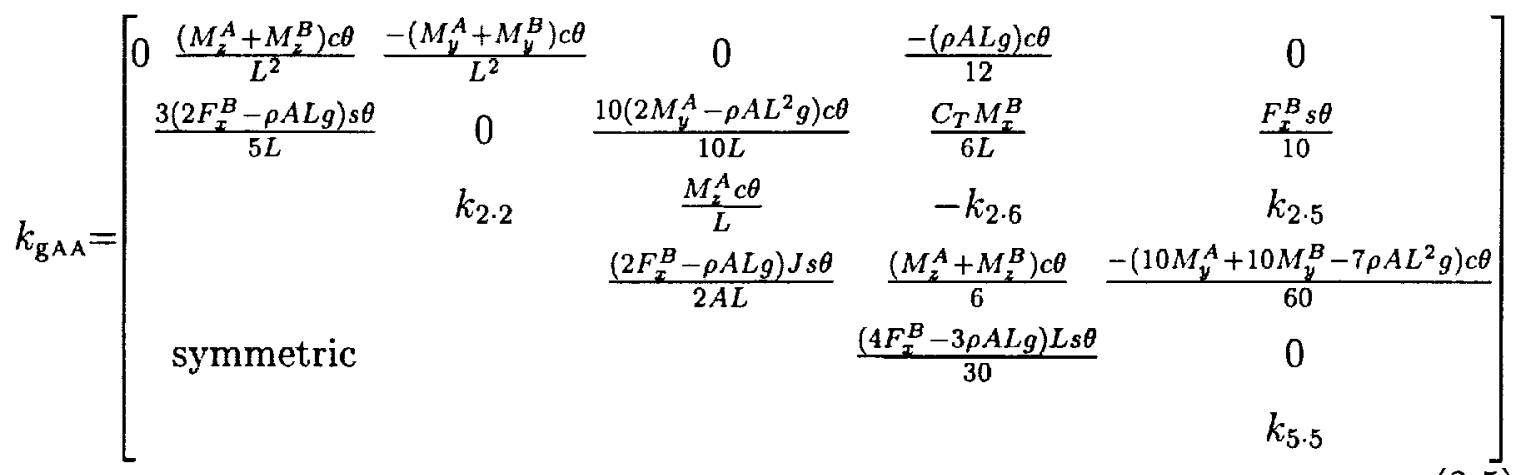

where $c \theta$ is $\cos \theta, s \theta$ is $\sin \theta$, and $\theta$ is the the angle between the horizontal and the long axial of the beam, as shown in Figure 2.5.

In comparing the original stiffness matrix in Equation 2.3 with that of the gravity adjustment stiffness matrix of Equation 2.5, there are a few couplings which to ob- 
serve. An axial load $\left(F_{x}\right)$ stiffens or de-stiffens all degrees of freedom except the axial, while also coupling the bending and axial degrees of freedom. A bending moment $\left(M_{y}, M_{z}\right)$ couples the axial and torsion degrees of freedom with the bending degree of freedom, while a torsional moment couples the out of plane bending degrees of freedom. The distributed loading, which is closest to a gravity loading, stiffens or de-stiffens all bending and torsion degrees of freedom, while also coupling axial and torsional degrees of freedom with the out of plane bending degree of freedom.

Another gravity effect is that of initial deformation in the form of sag (droop) or twist of the system. Initial deformation of a system can be written as a transformation between the nominal and deformed system, or

$$
K_{\text {def }}=T^{T} k T
$$

Again examining the 1-6 portion of the system, the transformation matrices for sag and twist are

$$
\begin{aligned}
& T_{\mathrm{SagAA}}=\left[\begin{array}{cccccc}
c \theta & 0 & -s \theta & & & \\
0 & 1 & 0 & & 0 & \\
s \theta & 0 & c \theta & & & \\
& & & c \theta & 0 & -s \theta \\
& 0 & & 0 & 1 & 0 \\
& & & s \theta & 0 & c \theta
\end{array}\right] \\
& T_{\mathrm{twiAA}}=\left[\begin{array}{cccccc}
1 & 0 & 0 & & & \\
0 & c \theta & s \theta & & 0 & \\
0 & -s \theta & c \theta & & & \\
& & & 1 & 0 & 0 \\
& 0 & & 0 & c \theta & s \theta \\
& & & 0 & -s \theta & c \theta
\end{array}\right]
\end{aligned}
$$

where $s \theta$ and $c \theta$ are $\sin \theta$ and $\cos \theta$ respectively.

The drooping of a system as in Equation 2.7 has the effects of coupling the axial and vertical bending degrees of freedom, and coupling the torsion and bending degrees 
of freedom. An initial twist about the neutral axis of the system, given in Equation 2.8 essentially couples the out of plane bending degrees of freedom.

\subsubsection{Testing Uncertainties}

Depending upon the type of testing, many uncertainties can also enter during the tests. Issues such as noise, bias, and bandwidth in sensors and time delays in computers can all can impede accurate measurements of the system. Strain gauges are one example of a sensor which is also very dependent on the attachment to the system. Also, unknown disturbances such as aerodynamics can lead to many uncertainties in the model. Additional testing uncertainties can arise when using shakers and accelerometers for modal analysis. The addition of these sensors and actuators should be modeled and parameterized in the identification process. Using actuators and sensors of good quality, and modeling all actuators, sensors and time delays thoroughly is the simplest step in avoiding these many types of uncertainties.

\subsubsection{Nonlinearities}

Nonlinearities in some form act on every system, and usually only hinder the uncertainty localization process. One of the primary objectives of using variance uncertainties in addition to mean uncertainties is to enable the localization of weak nonlinearities. Strong nonlinearities only degrade the uncertainty localization process, and usually require a nonlinear model, in addition to the finite element model.

Nonlinearities in a system can include material nonlinearities, stiction, dead band stiffness in joints, and changing boundary conditions such as loss of tension in a cabled suspension system. There are four manners in which to treat nonlinearities in a system: ignore them; use uncertainty localization techniques if they are weak; add additional components which downgrade a strong nonlinearity to a weak one, such as using a servo controller or DC bias; add a nonlinear model to the system. The work in this thesis attempts to address the second option. 


\begin{tabular}{|c|c|c|c|c|}
\hline \multirow[b]{2}{*}{ Group } & \multirow[b]{2}{*}{ Sub-group } & \multirow[b]{2}{*}{ Type } & \multicolumn{2}{|c|}{ Capturable } \\
\hline & & & Easy & Difficult \\
\hline \multirow{6}{*}{ Linear } & Physical Parameters & Material & $\checkmark$ & \\
\hline & & Geometric & $\sqrt{ }$ & \\
\hline & Modeling & $\begin{array}{l}\text { Discretization } \\
\text { Incorrect Element Physics }\end{array}$ & & $\begin{array}{l}\sqrt{ } \\
\sqrt{ }\end{array}$ \\
\hline & Internal Coupling & $\begin{array}{l}\text { Boundary Conditions } \\
\text { Joints/Attachments } \\
\text { Actuator/Sensor Dynamics } \\
\text { Time Delays }\end{array}$ & $\begin{array}{l}\sqrt{ } \\
\sqrt{ } \\
\sqrt{ } \\
\sqrt{ }\end{array}$ & \\
\hline & External Coupling & $\begin{array}{l}\text { Aerodynamic } \\
\text { Gravity } \\
\text { Control System }\end{array}$ & $\begin{array}{l}\sqrt{ } \\
\sqrt{ }\end{array}$ & $\sqrt{ }$ \\
\hline & Testing & $\begin{array}{l}\text { Sensor/Process Noise } \\
\text { Sensor Bias }\end{array}$ & $\sqrt{ }$ & $\sqrt{ }$ \\
\hline \multirow[t]{2}{*}{ Nonlinear } & Weak & & $\sqrt{ }$ & \\
\hline & Strong & & & $\sqrt{ }$ \\
\hline
\end{tabular}

Table 2.5: Table of uncertainties between structural hardware and models, along with the ability to capture them using identification and/or localization methods.

\subsubsection{Summary}

Table 2.5 gives a summary of the uncertainties that may exist between a structural model and set of hardware. In addition, the capturability, or ability of the tests and methods in this work to develop a physical mean or variance uncertainty which is the source of the uncertainty, is given as either easy or difficult. Physical uncertainties, for instance, are usually easy to localize. This is intuitively correct because of the wide use of model updating techniques available for these parameters. Modeling uncertainties, however, are far more difficult to capture with these techniques, primarily because the dynamics have not been captured correctly. For instance, if the wrong type of discretization is used, it will be difficult to localize these to uncertainties to correct physical degrees of freedom.

Internal couplings such as such as boundary conditions and actuator/sensor dynamics and joints and attachments can be localized by using the identification algo- 
rithm, rather than a finite element error localization technique. However, this can only be done if they are modeled and appended to the finite element model. Uncertainties in these dynamics can then be localized by parameterizing them in the identification process. External couplings complicate the system quite easily. Therefore, they must be examined case by case. Gravity couplings, for instance, are usually captured in the finite element model by an adjustment to the stiffness matrix. Therefore, they lend themselves to uncertainty localization.

It is difficult to know if testing uncertainties and nonlinearities can be represented with a physical uncertainty. It is assumed that the noise uncertainties, because they are small, will manifest themselves as larger variance uncertainties. Random bias errors that are large, however, cannot be represented as a linear physical change to the system. Many of these uncertainties can be avoided by using quality actuators and sensors, modeling them correctly.

Nonlinearities, however, can have the most detrimental effects on the localization process, especially if they are strong. If they are weak, or can be made weak by the addition of another component to the system such as a controller, these types of nonlinearities are assumed to be identified using variance uncertainties. If the nonlinearities are strong, a nonlinear model must be included.

In order to successfully identify and localize uncertainties, steps must be taken to reduce the effects of those uncertainties which are not capturable. These include:

- Refining the finite element mesh to prevent discretization and joint and/or attachment errors.

- Understanding the physics of the hardware such that the correct element types are used.

- Completely modeling boundary conditions and actuator and sensor dynamics.

- Completely understanding all coupling effects, and their impact on the finite element model.

- Completely understanding all nonlinear effects, including modeling all moderately nonlinear effects.

Once these precautions have been taken, the uncertainty localization process has the best chance of capturing the most accurate mass and stiffness uncertaintics. 


\section{Chapter 3}

\section{Identification of Nominal}

\section{Uncertainty Model}

In the previous chapter, a complete description was given of uncertainties that may exist between a finite element model a set of hardware. With these identified, the next step is to create and implement a set of tests which will allow the measurement of all uncertainties. This chapter gives a complete set of tests for hardware such that the uncertainties in Table 2.5 are observable and can be localized. A description is then given of the Discrete Extended Kalman Filter, the recursive identification/parameter estimation algorithm used to identify the modal parameters for each test. Once the identifications are complete for all tests, the estimated parameters can be used for creating accurate control design and uncertainty models for the 1-g system, termed "Nominal", and/or projection into 0-g, termed "Modified," for control design (Chapter 4). Finally, each of these techniques including testing, identification and estimation of parameters, and creation of control design and uncertainty models in 1-g, are demonstrated for the MACE experiment. 


\subsection{Testing for Uncertainty Identification}

Once the uncertainties between the finite element model and hardware have been evaluated, and the precautions have been taken to reduce those uncertainties that do not lend themselves for localization, the next step is to develop a set of tests which best observes all other possible uncertainties. These tests vary all parameters which may lead to either mean error or variance uncertainties between the model and hardware. Table 3.1 gives a summary of all test parameters that could be varied. Those tests that aid in the development of an uncertainty model by observing mean error and variance uncertainties of the nominal model described in Table 2.5 are denoted "Uncertainty." Those tests which require a change to the system, such that there is a fundamental change to the finite element model, are termed "Update."

The simplest and easiest uncertainty test is using a different actuator for the disturbance. Observing sensors using different disturbance source locations on a structure increases the number of parameters being identified and observes both physical modeling uncertainties and the linearity of the structure. Other uncertainty tests include disassembly/reassembly, allowing long periods of time between tests, and changing disturbance levels. Disassembly/reassembly changes the loading in the joints and members, a possible source of weak nonlinearities. Nonlinearities can also usually be detected by changing the disturbance level. This is especially true in cases of nonlinear damping. Also, by allowing longer time between tests, small changes in the structure can be detected such as plastic rather than elastic sag in a flexible member.

Other tests which are usually used for updating smaller portions of the finite element model are boundary condition changes and deterministic changes such as adding a known mass (or stiffness) to the structure. Wada et al. developed the most well-know of these called the Multiple Boundary Condition (MBC) tests [54], where the structure and gravity effects are modeled and updated by changing the boundary conditions, remodeling, retesting, and updating. These tests are excellent for model updating, but need to be adapted for uncertainty localization and projection as described in this work. Because there is usually a fundamental change to the 


\begin{tabular}{|l|c|c|}
\hline \multirow{2}{*}{ Test Parameters } & \multicolumn{2}{|c|}{ Use of Test } \\
\cline { 2 - 3 } & Uncertainty & Update \\
\hline \hline Actuators & $\sqrt{ }$ & \\
Disassembly/Reassembly & $\sqrt{ }$ & \\
Time between tests & $\sqrt{ }$ & \\
Disturbance Levels & $\sqrt{ }$ & \\
Boundary Conditions & & $\sqrt{ }$ \\
Structural Change (i.e. add mass) & & $\sqrt{ }$ \\
Non-Structural Change (i.e. closed loop) & $\sqrt{ }$ & \\
\hline
\end{tabular}

Table 3.1: Set of test parameters and whether they are primarily used for the development of an uncertainty model, or finite element model updating.

nominal finite element model for these tests, the frequency and mode shape information cannot be included in the nominal uncertainty model, which is based on the nominal finite element model. This information can only be added into the uncertainty model-localization-projection process at the localization level as uncertainties in specific portions of the model.

A final uncertainty test is termed system change and can also be used to develop the nominal uncertainty model. An example of this type of test parameter is the addition of a control system to the hardware. Care must be taken, however, in using a control system because of its effects on other uncertainties. The addition of a control system may increase effects such as nonlinearities, which would degrade the accuracy of the uncertainty model.

If the necessary precautions are taken in modeling of a system such as mesh refinement and modeling of actuators and sensors, and a complete set of tests such as those in Table 3.1 are run to thoroughly observe all uncertainties, the uncertainty localization procedure has the best possible chances of accurately predicting the complete uncertainty model. The next step is to identify the modal parameters from each of the tests. 


\subsection{Discrete Extended Kalman Filter}

There are many identification techniques which can be used to fit mathematical models to test data. Because of the special nature of this work, a technique is needed which enables the easy extraction of accurate modal parameters such as frequencies and normal modes from measurement models, and an easy parameterization such that a finite element model can be combined with actuator and sensor dynamics, time delays, and closed loop dynamics. The technique proposed in this work to identify the system is the Discrete Extended Kalman Filter (DEKF) [57]. The DEKF satisfies each of these requirements and in addition, the DEKF gives additional information on the estimation of the modal parameters in the form of a measurement error from the covariance matrix. Drawbacks to this technique include the use of an initial parameterized model and covariance, and convergence and bias problems. Each of these drawbacks are addressed in this chapter.

\subsubsection{Finite Element Based DEKF}

The DEKF is essentially a nonlinear filter which is linearized and updated to a standard Kalman Filter at each time step. Karlov et al. [6] developed the continuous time version of the Extended Kalman Filter and applied it to a lightly damped structure for identification and parameter estimation in control design. Because identification of a system is usually discrete in nature, and higher order filters are an easy and natural extension, the Discrete Extended Kalman Filter (DEKF) is developed here.

It is assumed that a finite element model has been developed for the system of the form

$$
\begin{aligned}
M \ddot{\eta}+C \dot{\eta}+K \eta & =B_{u} u \\
y & =C_{y} \eta+v
\end{aligned}
$$

where $M, C$, and $K$ are the mass, damping, and stiffness matrices respectively, $\eta$ is a vector of $n$ physical degrees of freedom, $u$ and $y$ are the inputs and outputs of the system, $v$ is the sensor noise, and $B_{u}$ and $C_{y}$ are input and output pickoff 
matrices. Note that the outputs are assumed to be displacements, although rates and accelerations are simple extensions. Using any commercially available finite element software, the undamped, no noise, free vibration case is solved as

$$
M \Phi \Lambda+K \Phi=0
$$

where $\Lambda$, and $\Phi$ are the $m$ eigenvalues and eigenvectors of the system (usually $m<n$ ). The eigenvectors are usually mass normalized such that

$$
\begin{aligned}
\Phi^{T} M \Phi & =I \\
\Phi^{T} K \Phi & =\Lambda
\end{aligned}
$$

Using a change of basis $\eta=\Phi q$ and Equation 3.3, and premultiplying by $\Phi^{T}$, Equation 3.1 yields

$$
\begin{aligned}
\ddot{q}+\Phi^{T} C \Phi \dot{q}+\Lambda q & =\Phi^{T} B_{u} u \\
y & =C_{y} \Phi q+v
\end{aligned}
$$

Assuming proportional damping, the system becomes

$$
\begin{aligned}
\ddot{q}+2 Z \Omega \dot{q}+\Omega^{2} q & =\Phi^{T} B_{u} u \\
y & =C_{y} \Phi q+v
\end{aligned}
$$

where

$$
\begin{aligned}
& Z=\operatorname{diag}\left\{\zeta_{i}\right\} \\
& \Omega=\operatorname{diag}\left\{\sqrt{\lambda_{i}}\right\}
\end{aligned}
$$

are the frequencies and damping ratios of the system. Rewriting Equation 3.5 (in the traditional $2^{\text {nd }}$ order form) in $1^{\text {st }}$ order or state space form

$$
\begin{aligned}
{\left[\begin{array}{c}
\dot{q} \\
\ddot{q}
\end{array}\right] } & =\left[\begin{array}{cc}
0 & I \\
-\Omega^{2} & -2 Z \Omega
\end{array}\right]\left[\begin{array}{l}
q \\
\dot{q}
\end{array}\right]+\left[\begin{array}{c}
0 \\
\Phi^{T} B_{u}
\end{array}\right] u \\
y & =\left[\begin{array}{ll}
C_{y} \Phi & 0
\end{array}\right]\left[\begin{array}{l}
q \\
\dot{q}
\end{array}\right]+v
\end{aligned}
$$


In proceeding further with the derivation of the DEKF, a few notational simplifications are used without loss of generality. First, because the system in Equation 3.6 is decoupled into modes, a single mode is used. It is also assumed that the input and output matrices, $B_{u}$ and $C_{y}$, use only one degree of freedom. And finally, a single input and single output are used. Additional modes, linear combinations of modes within the input and/or output matrices, and additional inputs and outputs are assumed to be simple extensions. Therefore, the system becomes

$$
\begin{aligned}
& \dot{x}_{i}=A_{i} x_{i}+b_{i j} u_{j} \\
& y_{k}=c_{k i} x_{i}+v_{k}
\end{aligned}
$$

where the $i^{\text {th }}$ mode, $j^{\text {th }}$ input, and $k^{\text {th }}$ output are used and

$$
\begin{aligned}
A_{i} & =\left[\begin{array}{cc}
0 & 1 \\
-\omega_{i}^{2} & -2 \zeta_{i} \omega_{i}
\end{array}\right] \\
b_{i j} & =\left[\begin{array}{c}
0 \\
\phi_{j i} b_{u j}
\end{array}\right] \\
c_{k i} & =\left[\begin{array}{ll}
c_{y k} \phi_{k i} & 0
\end{array}\right] \\
x_{i} & =\left[\begin{array}{c}
q_{i} \\
\dot{q}_{i}
\end{array}\right]
\end{aligned}
$$

Note that $b_{u j}$ and $c_{y k}$ are constants.

It is also assumed that the input enters as a zero order hold process in the identification experiment. The continuous time system in Equation 3.7 can be converted to discrete time using the matrix exponential [58].

$$
\begin{aligned}
& A_{\mathrm{d} i}=e^{A_{i} d t} \\
& b_{\mathrm{d} i j}=A_{i}^{-1}\left(A_{\mathrm{d} i}-I\right) b_{i j}
\end{aligned}
$$

This assumes there is no delay between the sensor measurement and control input except for the $1 / 2$ cycle from the zero order hold. Additional delays can be easily added to the system, see Ref. 58. The output equation does not change.

In a general system with multiple modes, inputs, and outputs, and sensor/actuator dynamics and time delays, there are many different parameters that can be estimated. 
For the uncertainty model (and subsequent uncertainty localization and projection problems), the parameters of concern are the frequencies, damping ratios, and normal modes. A more general system is addressed in the next section. Therefore, the parameters of the system that are updated are defined as

$$
\theta=\left[\begin{array}{llll}
\omega_{i} & \zeta_{i} & \phi_{j i} & \phi_{k i}
\end{array}\right]^{T}
$$

If a state vector at time $t$ is defined as

$$
z(t)=\left[\begin{array}{c}
x_{i}(t) \\
\theta(t)
\end{array}\right]
$$

the DEKF equations are

$$
\begin{aligned}
\hat{z}(t+1 \mid t) & =A_{\mathrm{d} i}(t) x_{i}+b_{\mathrm{d} i j}(t) u_{j}(t) \\
\hat{z}(t+1 \mid t+1) & =\hat{z}(t+1 \mid t)+K(t+1) \nu(t+1) \\
\nu(t+1) & =y_{k}(t+1)-C(t+1) \hat{z}(t+1 \mid t) \\
P(t+1 \mid t) & =A(t) P(t \mid t) A(t)^{T} \\
P_{\nu}(t+1 \mid t) & =C(t+1) P(t+1 \mid t) C(t+1)^{T}+\Sigma_{v v} \\
P(t+1 \mid t+1) & =P(t+1 \mid t)-K(t+1) C(t+1) P(t+1 \mid t) \\
K(t+1) & =P(t+1 \mid t) C(t+1)^{T} P_{\nu}(t+1 \mid t)^{-1}
\end{aligned}
$$

where

$$
\begin{aligned}
A_{\mathrm{d} i}(t) & =\left.A_{\mathrm{d} i}\right|_{\theta=\theta(t \mid t)} \\
b_{\mathrm{d} i j}(t) & =\left.b_{\mathrm{d} i j}\right|_{\theta=\theta(t \mid t)} \\
c_{k i}(t+1) & =\left.c_{k i}\right|_{\theta=\theta(t+1 \mid t)} \\
A(t)= & {\left[\begin{array}{cc}
A_{\mathrm{d} i}(t) & \left.\frac{\partial}{\partial \theta}\left(A_{\mathrm{d} i} x_{i}+b_{\mathrm{d} i j} u_{j}(t)\right)\right|_{\theta=\theta(t \mid t), x_{i}=x_{i}(t \mid t)} \\
0 & I
\end{array}\right] } \\
\text { and } & C(t+1)=\left[\begin{array}{cc}
c_{k i}(t+1) & \left.\frac{\partial}{\partial \theta}\left(c_{k i} x_{i}\right)\right|_{\theta=\theta(t+1 \mid t), x_{i}=x_{i}(t+1 \mid t)}
\end{array}\right]
\end{aligned}
$$

The parameters are assumed to be constant over the time of the identification, and the system contains no process noise. Notice that although the algorithm is discrete 
in nature, the parameters that are updated are from the physical continuous model. These parameters can then be used for model updating, development of an uncertainty model, and/or control design.

\subsubsection{Additional Dynamics}

The derivation of the DEKF in Section 3.2.1 uses a single mode, and single input and output. Multiple modes, inputs, and outputs are assumed to be simple extensions. For more practical systems, however, additional dynamics should be included in the system such as actuator and sensor dynamics, time delays, and even closed loop dynamics. Dropping the $(\cdot)_{i}$ 's from the nominal finite element model in Equation 3.7, and discretizing using a zero order hold,

$$
\begin{aligned}
& \dot{x}=A_{\mathrm{d}} x+b_{\mathrm{d}} u \\
& y=c x+v
\end{aligned}
$$

Dynamics on the inputs and outputs are easily appended to the finite element model.

$$
\begin{aligned}
& {\left[\begin{array}{c}
\dot{x} \\
\dot{x}_{i} \\
\dot{x}_{o}
\end{array}\right]=\left[\begin{array}{ccc}
A_{\mathrm{d}} & b_{\mathrm{d}} c_{o} & 0 \\
0 & A_{i} & 0 \\
b_{o} c & 0 & A_{o}
\end{array}\right]\left[\begin{array}{c}
x \\
x_{i} \\
x_{o}
\end{array}\right]+\left[\begin{array}{cc}
b_{\mathrm{d}} & 0 \\
0 & b_{i} \\
0 & 0
\end{array}\right]\left[\begin{array}{c}
u \\
u_{i}
\end{array}\right]} \\
& {\left[\begin{array}{c}
y \\
y_{o}
\end{array}\right]=\left[\begin{array}{ccc}
c & 0 & 0 \\
0 & 0 & c_{o}
\end{array}\right]\left[\begin{array}{c}
x \\
x_{i} \\
x_{o}
\end{array}\right]+\left[\begin{array}{c}
v \\
v_{o}
\end{array}\right]}
\end{aligned}
$$

where ()$_{i}$ and ()$_{o}$ refer to the dynamics on the inputs and outputs respectively. The DEKF can now be developed in an analogous manner to that in Equations 3.93.11 using this new parameterization. In addition, parameters of the dynamics on the inputs and outputs can easily be added to the DEKF by adding them to the parameter vector in Equation 3.9 and taking the appropriate derivatives in the derivation.

A closed loop system can also be easily added to the parameterization. If a dynamic compensator is used, it can be appended to the system in an analogous manner to the output dynamics in Equation 3.13. Then the system is closed to 
create a new parameterization. For example, if constant output feedback is used, the system becomes

$$
\begin{aligned}
& \dot{x}=\left(A_{\mathrm{d}}-b_{\mathrm{d}} c\right) x+b_{\mathrm{d}} u \\
& y=c x+v
\end{aligned}
$$

The DEKF can the be developed analogous to that in Equations 3.9-3.11.

\subsubsection{Initialization}

Although the DEKF has the advantage of having a parameterization that is flexible enough to include the dynamics of components such as the finite element model and actuator and sensor dynamics, this is also a disadvantage. The initial model must be a fairly accurate representation of the data. If the DEKF is used to identify a single model, any initial parameterized model can be used. If it is used for multiple identifications, however, for combination into an uncertainty model, the same model and parameterization must be used for each identification. The model and DEKF which present the easiest, most intuitive results is the finite element model derivation presented in the previous section.

In addition, the initial covariance matrix and noise must be chosen. Initialization of the covariance and noise intensity matrices must be done using insight of the user. For the technique presented that uses no process noise, the quality of the final parameter estimates is rarely dependent upon the initialization of these matrices. When the DEKF actually has process noise, however, the final parameter estimates are biased [59]. A few precautions can be taken prior to using the DEKF to prevent convergence and bias problems. These are:

- A good initial model, i.e. frequency errors less than $10 \%$, damping errors less than $50 \%$, and mode shape errors less than $50 \%$.

- Initialize the covariance matrix with $3 \sigma$ error values less than the actual errors, i.e. frequency $3 \sigma$ of $10 \%$, damping $3 \sigma$ of $50 \%$, and mode shape $3 \sigma$ of $50 \%$.

- Initialize the noise intensity to approximate physical values, and vary if convergence rate is too fast/slow. 


\subsubsection{Convergence}

An excellent treatment of the convergence and bias properties of the DEKF is given in Ref. 59. The convergence of the general DEKF algorithm is not guaranteed. However, the case presented in the this work contains no process noise, even though there is a deterministic input, and is therefore quite general for identification purposes and guarantees can be made on convergence. Ljung [60] analyzes the properties of recursive stochastic algorithms including the Discrete Extended Kalman Filter [59]. This is done by examining the stability properties of an associated differential equation. For the DEKF, Equation 3.11 is examined assuming the parameters $\theta=$ constant.

For the case of no process noise, termed a "deterministic model" case by Ljung [59], convergence of the filter is guaranteed if it is equipped with a projection facility to keep $\theta(t)$, in a compact subset of $\left\{\theta \mid A_{\mathrm{d} i}(t)\right.$ stable $\}$. For an open loop system, this is easily accomplished by bounding the allowable parameter space to be finite, and keeping $A_{\mathrm{di}}(t)$ stable by bounding the frequency and damping ratio parameters. For a closed loop system, this is more difficult, and care must be taken in implementing the projection facility. If these requirements are met, the estimate converges with probability 1 to a stationary point of the function

$$
V(\theta)=E\left[\nu(t)^{T} \Sigma_{v v}^{-1} \nu(t)\right]
$$

Although Equation 3.15 has only local minima for stationary points, the estimate can be proven to be a global minimum if there exists an estimate $\theta_{0}$ such that

$$
c_{k i}\left(\theta_{0}\right)\left(z I-A_{\mathrm{d} i}\left(\theta_{0}\right)\right)^{-1} b_{\mathrm{d} i j}\left(\theta_{0}\right)=c_{0}\left(z I-A_{0}\right)^{-1} b_{0}
$$

where $c_{0}, A_{0}$, and $b_{0}$ represent the "true" system. Equation 3.16 can be represented in another way by stating that the innovations $\nu(t)$ are unbiased, which is addressed in the next section. Although Equation 3.15 is not necessarily the objective of a Kalman Filter, minimizing this performance objective is a good relative measure of the ability of the estimation algorithm. 


\subsubsection{Bias}

In addition to there being no convergence guarantees for the DEKF, there are also no bias guarantees [59]. For the general DEKF, if the noise intensities are incorrect, the estimates are always biased. Unfortunately, there are no simplifying solutions for the deterministic model case for bias as there is for convergence. The estimates for the DEKF are said to be unbiased if the innovations are both unbiased and white. The following tests for these properties are taken from Refs. 61,62 . To rigorously prove that the innovations are unbiased, a $\chi^{2}$ test on the normalized innovations squared is used. To prove that the innovations are white, that is, uncorrelated in time, the autocorrelation function of the innovation sequence is calculated.

There are two tests given in Refs. 61,62, however, which are much easier to calculate and give a qualitative but not rigorous test for unbiasedness of the estimates. First, the noise intensity of the filter is checked by examining the innovations sequence. If $95 \%$ of the innovations lie within the $2 \sigma$-gate, then the noise intensities are set to acceptable levels. Then, the bias of the innovations will qualitatively tell whether the DEKF is biased. The DEKF is therefore said to be unbiased if the mean of the innovations $\nu$, is less than the $2 \sigma$-gate, or if $E[\nu]<0.02 \cdot\left[2 \sigma_{\nu}\right]$.

\subsubsection{Uniqueness}

Even if the algorithm is guaranteed to converge and be unbiased, the uniqueness of the solution is not necessarily guaranteed. An example of this can be seen by examining the original system in Equation 3.7. Equation 3.16 can be satisfied for this system for an infinite number of solutions because the $\phi_{i j}$ and $\phi_{k i}$ matrices can be scaled arbitrarily. If, however, one sensor and one actuator are collocated such that $\phi_{i j}=\phi_{k i}$ for the $i^{\text {th }}$ mode, $j^{\text {th }}$ input, and $k^{\text {th }}$ output, then the estimate is a global, unique minimum of Equation 3.16 and the estimate is unique. This is quite important when attempting to solve for the normal modes of multiple identifications, and then combining the information into an uncertainty model. 


\subsection{Uncertainty Model}

After performing the numerous tests on the hardware by varying the test parameters given in Table 3.1, and utilizing the DEKF algorithm to identify the modal parameters of the system, the next step is to combine this information into an uncertainty model. Note that this process is dependent upon the solution to the DEKF being unbiased and unique.

A stochastic uncertainty model is created by finding the statistics of the modal parameters, i.e. the eigenvalues and eigenvectors of the finite element model given in Equation 3.2, and the damping matrix given in Equation 3.5. Once the statistics of these parameters have been calculated, an accurate control design model can be created by shifting the original finite element model by the mean parameter uncertainties. In addition, the variances in the uncertainty model are used to create bounds on critical parameters for robust control design. This process is shown in Figure 1.1.

The DEKF provides additional information in the parameter estimation algorithm than just the estimates, in the form of a covariance matrix. In the Kalman Filter, this matrix is the on-line measurement error for the states. In the general DEKF with process noise on the parameters, the covariance matrix is directly related to the noise intensity given, and therefore, may be biased. In the deterministic case presented here, the covariance matrix tends to a steady state of 0 as the estimator proceeds in time, even if the estimates are biased. Therefore, the statement that the error matrix gives the absolute measurement error for the estimates is not correct. There is still good relative information in the error matrix, however. The measurement errors for each of the parameters act as confidence factors in developing the uncertainty model. For instance, the variance of parameters that are difficult to estimate tend to 0 at a slower rate than those that are not. Therefore, a measurement error that is large implies that the parameter is difficult to estimate, and therefore should be weighted less when creating the uncertainty model.

Defining the uncertainty $\Delta \theta_{j}$ as the difference between the estimate $\theta_{j}$ from the 
$j^{\text {th }}$ DEKF measurement model, and the initial parameter $\theta_{0}$.

$$
\Delta \theta_{j}=\theta_{j}-\theta_{0}
$$

The measured expected value and covariance of the errors can then be calculated.

$$
\begin{aligned}
\mathrm{E}[\Delta \theta] & =W^{-1} \sum_{j=1}^{n_{I}}\left[\Delta \theta_{j}\right] W_{j} \\
\operatorname{Cov}[\Delta \theta] & =(W-I)^{-1} \sum_{j=1}^{T}\left[\Delta \theta_{j}-\mathrm{E}[\Delta \theta]\right] W_{j}^{2}\left[\Delta \theta_{j}-\mathrm{E}[\Delta \theta]\right]^{T} \\
W_{j} & =\operatorname{diag}\left\{1 / \sigma_{j i}\right\} \\
W & =\sum_{j=1}^{T} W_{j}
\end{aligned}
$$

where $\sigma_{j i}$ represents the standard deviation of the measurement error from the DEKF for the $j^{\text {th }}$ estimate of the $i^{\text {th }}$ parameter, and $T$ is the total number of parameter estimates. From the expected value and covariance, the mean error, denoted $\overline{\Delta \theta}$ and bound, denoted $\widehat{\Delta \theta}$ can be readily derived

$$
\begin{aligned}
& \overline{\Delta \theta}=\mathrm{E}[\Delta \theta] \\
& \widehat{\Delta \theta}=3 \cdot \sqrt{\operatorname{diag}\{\operatorname{Cov}[\Delta \theta]\}}
\end{aligned}
$$

The bound is assumed to be three times the measured standard deviation of the

parameter. Assuming a normal distribution and the individual weights are $W_{j}=I$, a $99.5 \%$ confidence interval results for each parameter. Note that this number may change depending on the function of the uncertainty model (i.e. what are the effects of an unstable control system.) When using the stochastic uncertainties for projection, the mean and entire covariance matrix are used.

\subsection{MACE Results}

The Middeck Active Control Experiment (MACE) is used to show this process of creating an uncertainty model for the multiple uses of creating an accurate model for control design, robust control design, model updating, and uncertainty localization 
and projection. First, an examination of the hardware is made for likely uncertainties, and a set of tests are designed to observe these uncertainties. Then an example of the DEKF implemented on 1-g test data is given to show the practicality of this technique. Finally, the uncertainty model is developed from the multiple tests performed on MACE.

\subsubsection{Testing}

The first step in creating an uncertainty model is an evaluation of the uncertainties of the system, and the development of a test plan. In Section 2.2 .6 a checklist was presented to prevent those uncertainties which are difficult to localize from occurring in the system. These include: refining the finite element mesh, using the correct element types, and modeling of actuators, sensors, etc, and incorporation of coupling effects such as gravity. For MACE, these have been done, and are summarized in Ref. 39.

There could be many nonlinear effects, but the largest are likely due to the stiction of the gimbals and reaction wheels (assuming the rotations of the gimbals are small.) The large effects of these are relieved by the addition of servo controllers at the gimbals, and speed servos and a DC bias for each reaction wheel. It is assumed that these fixes have left the hardware with only weak nonlinearities.

Table 3.1 gives a summary of test parameters that can be varied in order to observe all possible modeling uncertainties. Note that multiple test parameters can be varied at the same time. For MACE, there were 24 distinct identifications performed, attempting each of the 7 test parameters in Table 3.1. There were 4 different actuators, and 17 different sensors used; 3 disassemblies and reassemblies; 1 suspension change; 1 change in disturbance levels; 1 deterministic change; and 1 system change. Table 3.2 gives a summary of the tests that were performed for MACE.

The deterministic change performed on MACE was the replacement of the active strut with a more flexible passive strut. The boundary condition change was an adjustment to the suspension system. Because these types of changes require a fundamental change to the finite element model, they were excluded from the develop- 


\begin{tabular}{|l|c|l|}
\hline Test Parameter & Number & Comment \\
\hline \hline Actuators & 4 & All gimbal actuators \\
Disassembly/Reassembly & 3 & including after 0-g flight \\
Time between tests & 4 & from 2 weeks to 3 months \\
Disturbance Levels & 1 & disturbance halved for 4 actuators \\
Boundary Conditions & 1 & change of suspension system \\
Deterministic Change & 1 & remove active strut \\
System Change & 1 & remove gimbal servos \\
\hline
\end{tabular}

Table 3.2: Set of tests performed on MACE to observe modal uncertainties and update the finite element model.

ment of the uncertainty model. The system change involved turning the gimbal servo systems off, creating a fully open loop system. This change, however, also increased the adverse nonlinear effects of stiction in the gimbals, thus giving quite different results. Because a nonlinear model is now required for this system to be modeled correctly, this test parameter was also not included in the development of the uncertainty model. These three test parameters affected 8 different identification data sets. Therefore, the total number of tests performed and utilized in the development of the MACE 1-g uncertainty model is 16.

\subsubsection{DEKF}

When the actuator and sensor dynamics, time delays, Bessel filters, and notch filters are added to the original 160 state finite element model, a 256 state input-output model is created. To reduce the large amount of computation time required by the DEKF, the size of this model is reduced. First, the Bessel filters and notch filters are modeled as time delays, which was a good approximation up to $100 \mathrm{~Hz}$. Of the 80 1-g modes, only 36 of those which are important to the input-output nature of the system are retained. This reduced the final model size to 96 states.

The damping in the input-output model is assumed to be proportional in order to create a block diagonal form. This has the benefit of reducing the number of internal calculations of the DEKF. Therefore, the concentrated dampers of the suspension 
system are lumped into the proportional damping. This change primarily affected the gimbal pendular modes, and is assumed to be negligible.

The input-output model created from the finite element model is a very good representation of the structure. Therefore, to initialize the DEKF, $3 \sigma$ values of $5 \%$, $50 \%$, and $50 \%$ are used for the frequencies, damping ratios, and mode shape entries respectively. Because of the difficulty in finding actual values for sensor noises, a noise to signal ratio of 0.2 is used for all sensors. Although this seems a bit ad hoc, changing these values did not have a large effect on the convergence of the DEKF.

Because the MACE test article easily decouples into two systems (see Figure 2.1), the $\mathrm{Z}$ axis and $\mathrm{XY}$ axes, the cross-coupling mode shape estimates are not parameterized in the DEKF because of the lack of observability. For instance, when the disturbance actuator is the Z-axis gimbal, only Z-axis sensors are used in the DEKF. The $\mathrm{X}$ and $\mathrm{Y}$ sensors are too noisy for good estimates. In addition, the heavily damped sensor dynamics and time delays that are appended to the system did not seem to effect the accuracy of the final solution, and are therefore excluded from the DEKF parameterization.

As an example of using the DEKF for a MACE identification, a bandlimited white noise disturbance enters into the X-axis primary gimbal (see Figure 2.1). There were 9 different sensors used: 4 strain gauges, 1 rate gyro in the primary payload and 2 rate gyros at the center node; and two encoders at the primary (collocated) and secondary payloads. There were 14 different modes identified, giving 154 parameters updated in the DEKF. (Note: one sensor and actuator are collocated, and therefore use the same parameter.) The experiment was performed for 360 seconds, and sampled at $500 \mathrm{~Hz}$.

Figure 3.1 gives a comparison of the FEM, data, and identified model for two pertinent transfer functions. As one can see, by varying only parameters of the FEM, an updated model is created which is an excellent representation of the data. Figure 3.2 shows the time history plots of the frequency and damping ratio for a mode at approximately $53.5 \mathrm{~Hz}$. The parameter estimates and $3 \sigma$ measurement crrors show the DEKF beginning to converge. As these time traces are taken out further, they do 


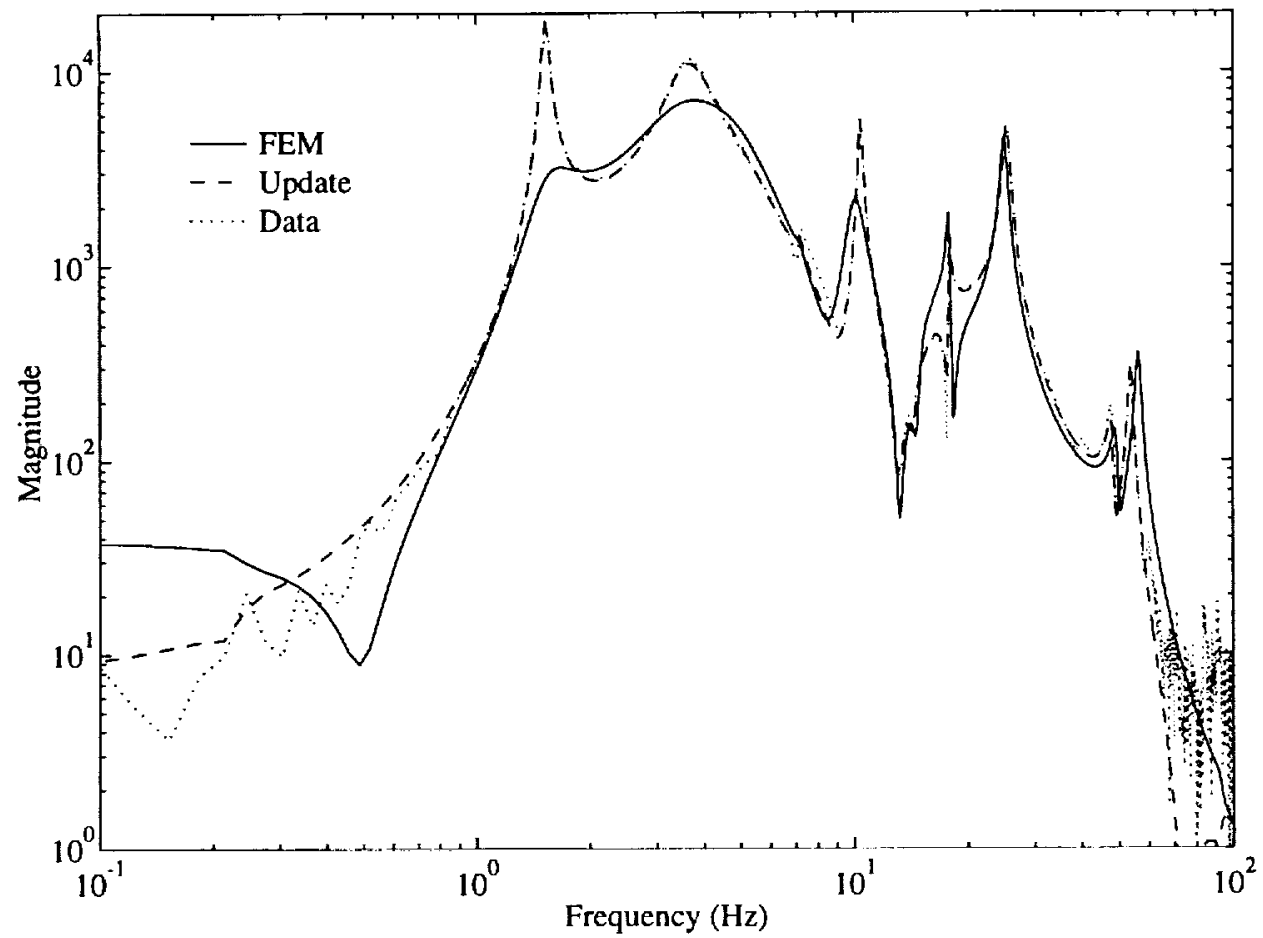

(a) Primary X Gimbal to Strut 3 X Strain Gauge.

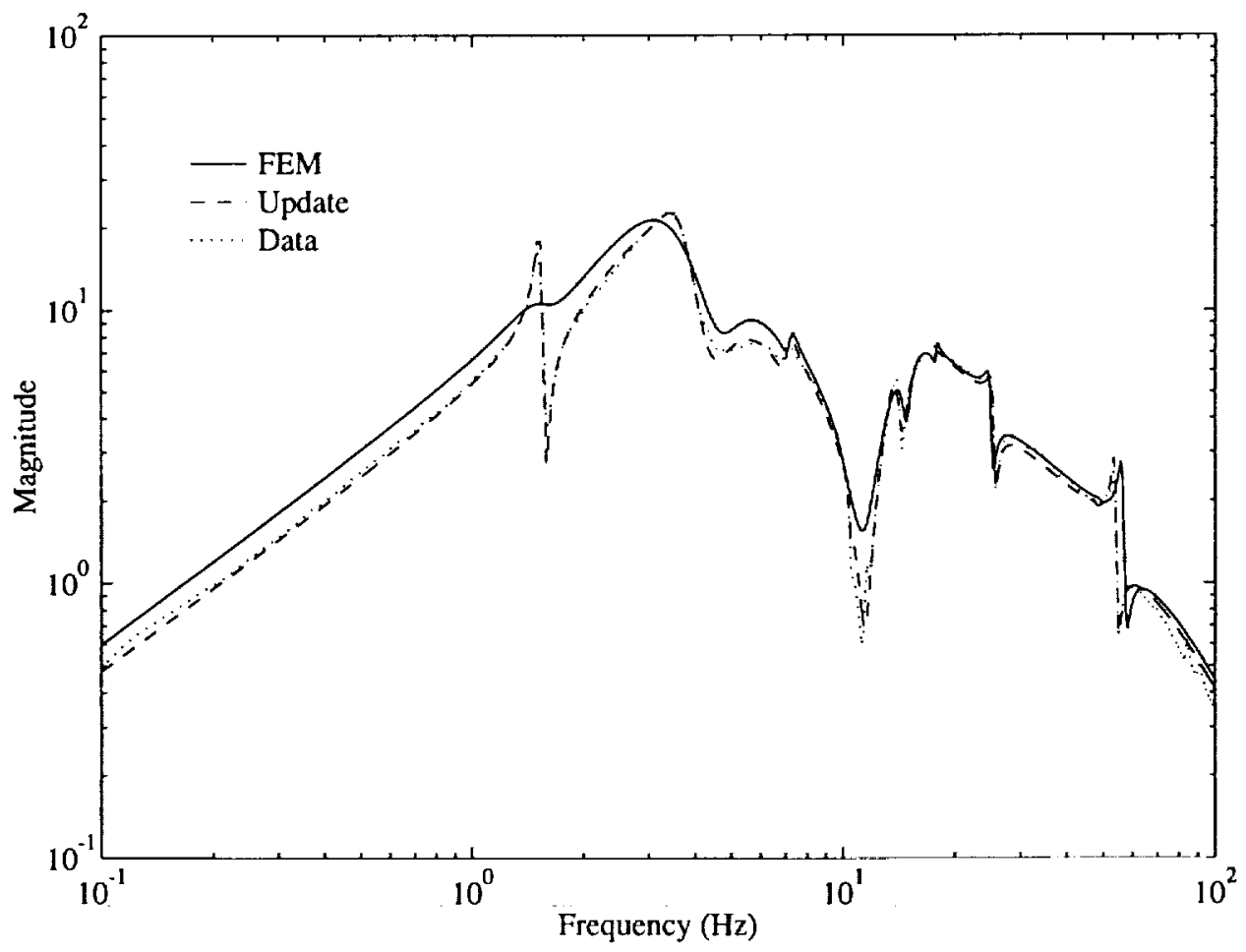

(b) Primary X Gimbal to Primary X Rate Gyro.

Figure 3.1: FEM, updated, and data transfer functions for a sample identification of the DEKF on MACE. 


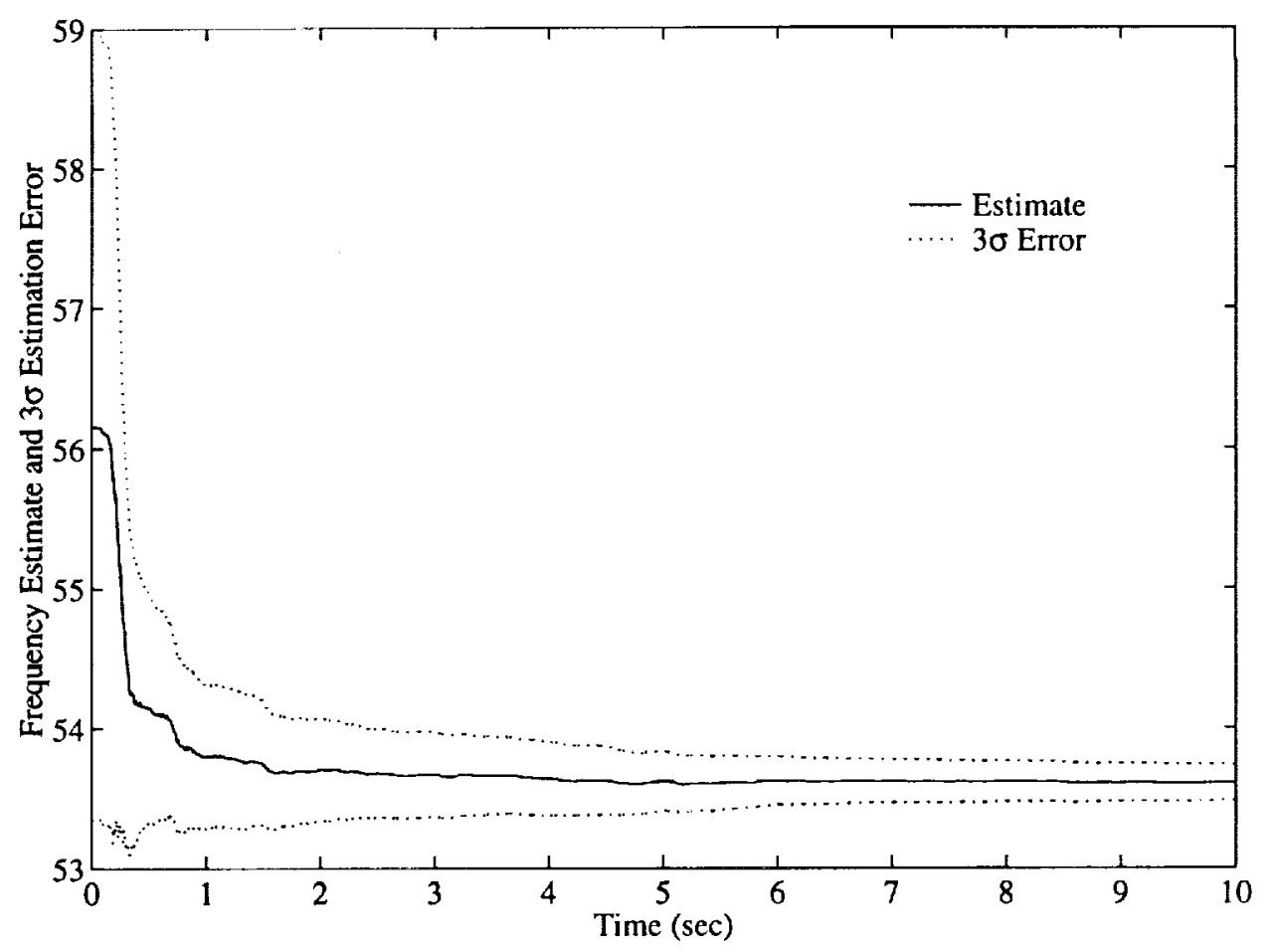

(a) Frequency estimate and $3 \sigma$ measurement error.

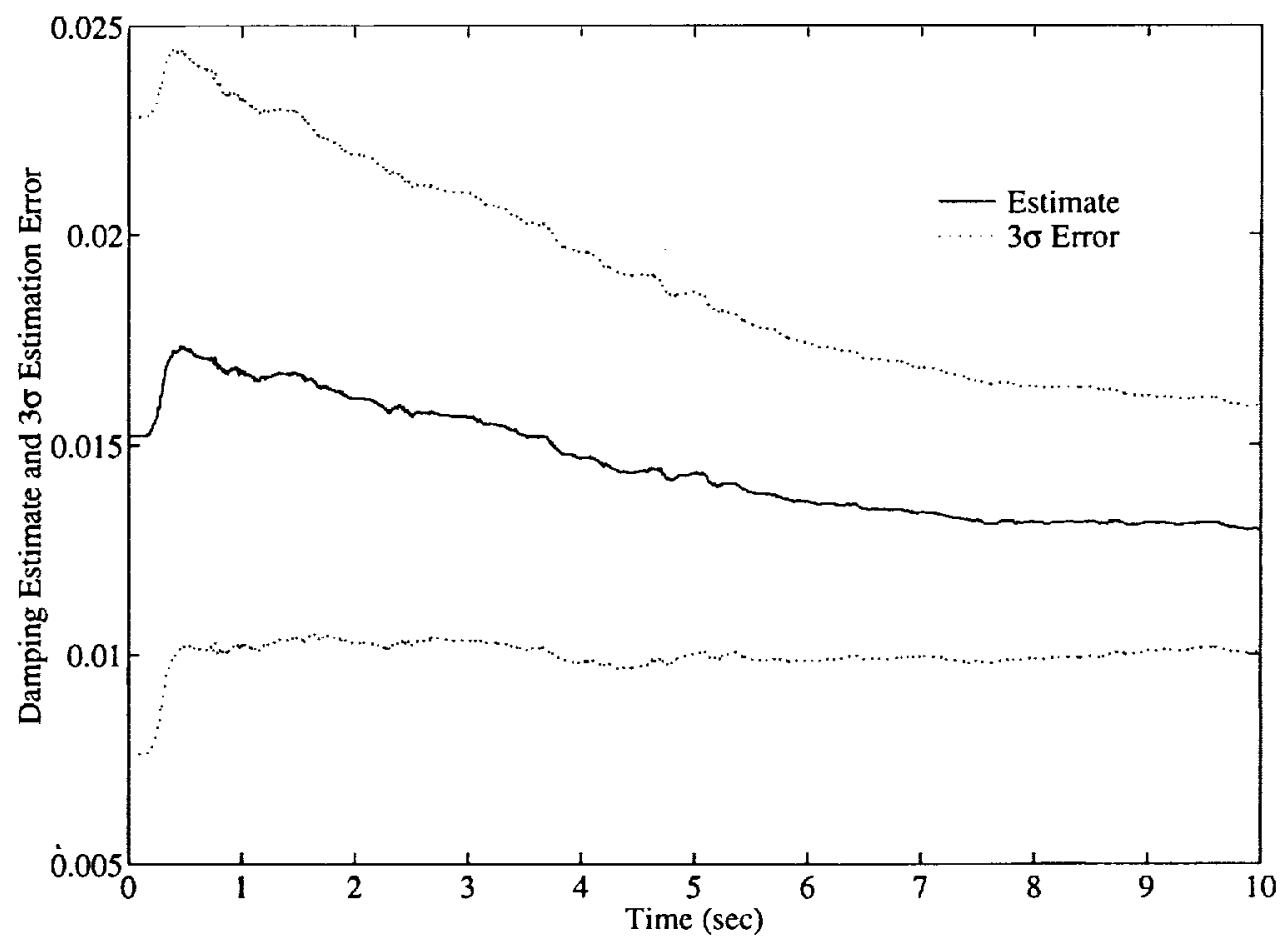

(b) Damping ratio estimate and $3 \sigma$ measurement error.

Figure 3.2: Time histories for the recursive DEKF, showing the convergence of frequency and damping ratio estimates. 
indeed converge. Notice that the frequency estimate and measurement error bound converge faster than those of the damping ratio estimate. This is intuitively correct because the damping is a much more difficult parameter to estimate. This shows the inherent value in the use of the $3 \sigma$ measurement error bounds in the development of the uncertainty model.

\subsubsection{1-g Uncertainty Model}

There were 16 identifications run for MACE, 8 which update the XY parameters, and 8 which update the $\mathrm{Z}$ parameters. Therefore, each parameter has 8 estimates to create the 1-g uncertainty model using Equations 3.21 and 3.21.

Figure 3.3(a) shows how this is done for a sample frequency and damping ratio using 8 data sets. The mean and bounds show how the weights of each of the identifications are incorporated into the uncertainty model. The estimates from each data set $(+)$ are weighted by the width of the grey column to create the mean $(x)$ and bound $(--)$ for the parameter. For the sample frequency, notice how the mean is heavily weighted by the "wide" estimates in the $53.6-53.8 \mathrm{~Hz}$ range. The two estimates which are greater than $54 \mathrm{~Hz}$ have much smaller weights, and therefore, the bound does not incorporate them. The same trends can be seen in the sample damping ratio case. However, notice how the larger weight on the estimate at 0.0225 creates a very large bound for this parameter.

Figure 3.3(b) shows an overlay of the frequency response of 3 data sets and the finite element model shifted by the mean errors of the uncertainty model, termed "shifted" model. The variations in each data set can easily be seen, especially near the $48 \mathrm{~Hz}$ and $54 \mathrm{~Hz}$ modes. The shifted model fits nicely as the "mean" of the data, while the finite element model contains relatively large errors. Using the shifted model and bounds on the critical parameters from the uncertainty model, any number of control design techniques can be used to design robust controllers for this system.

Table 3.3 shows the MACE 1-g uncertainty model, based on the 16 sets of parameter estimates. Notice that only the controllable/observable modes are updated and retained in the uncertainty model. Except for the gimbal pendular modes, the 

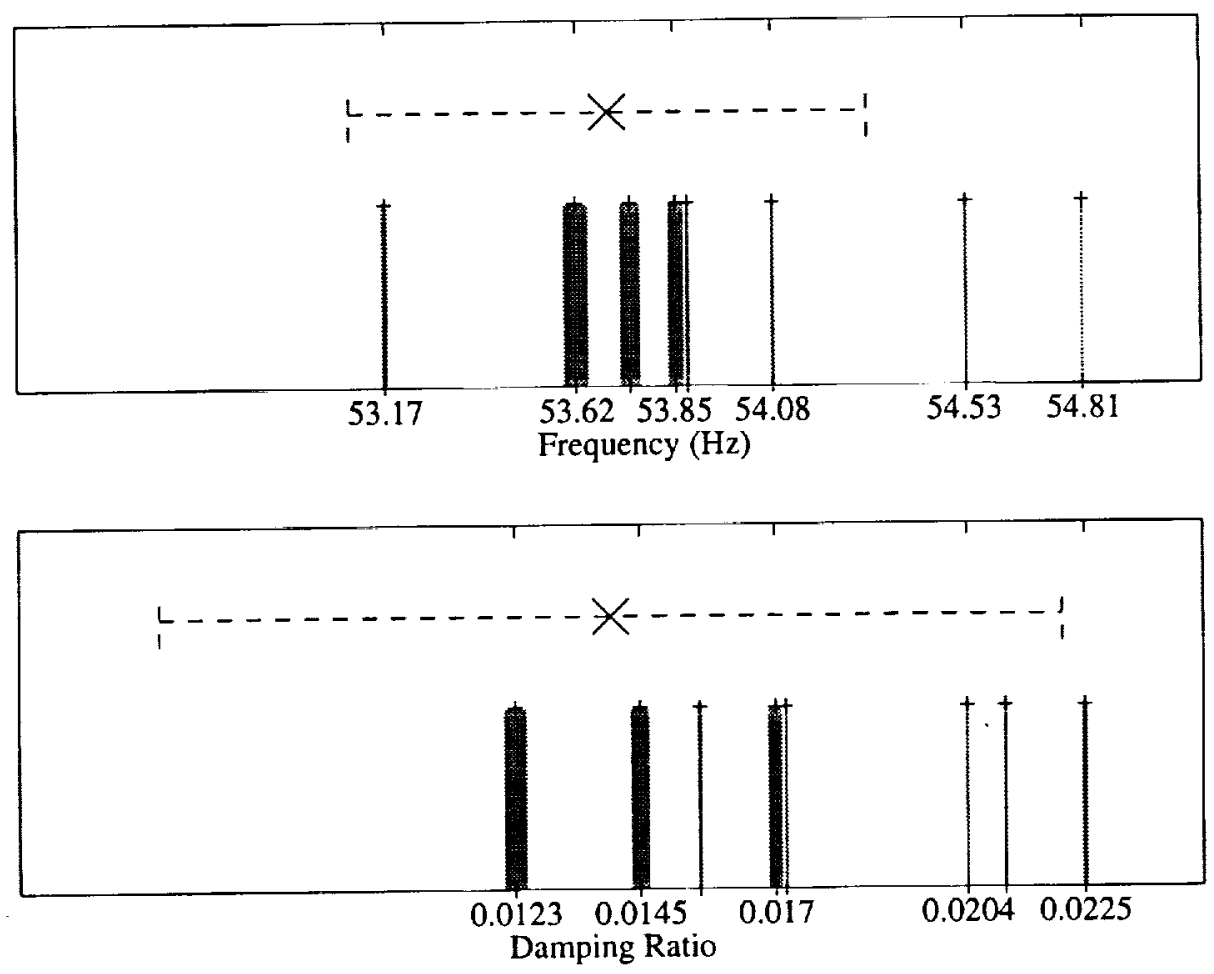

(a) Development of mean $(x)$ and bound $(--)$ for a sample frequency and damping ratio in the uncertainty model for multiple data sets $(+)$.

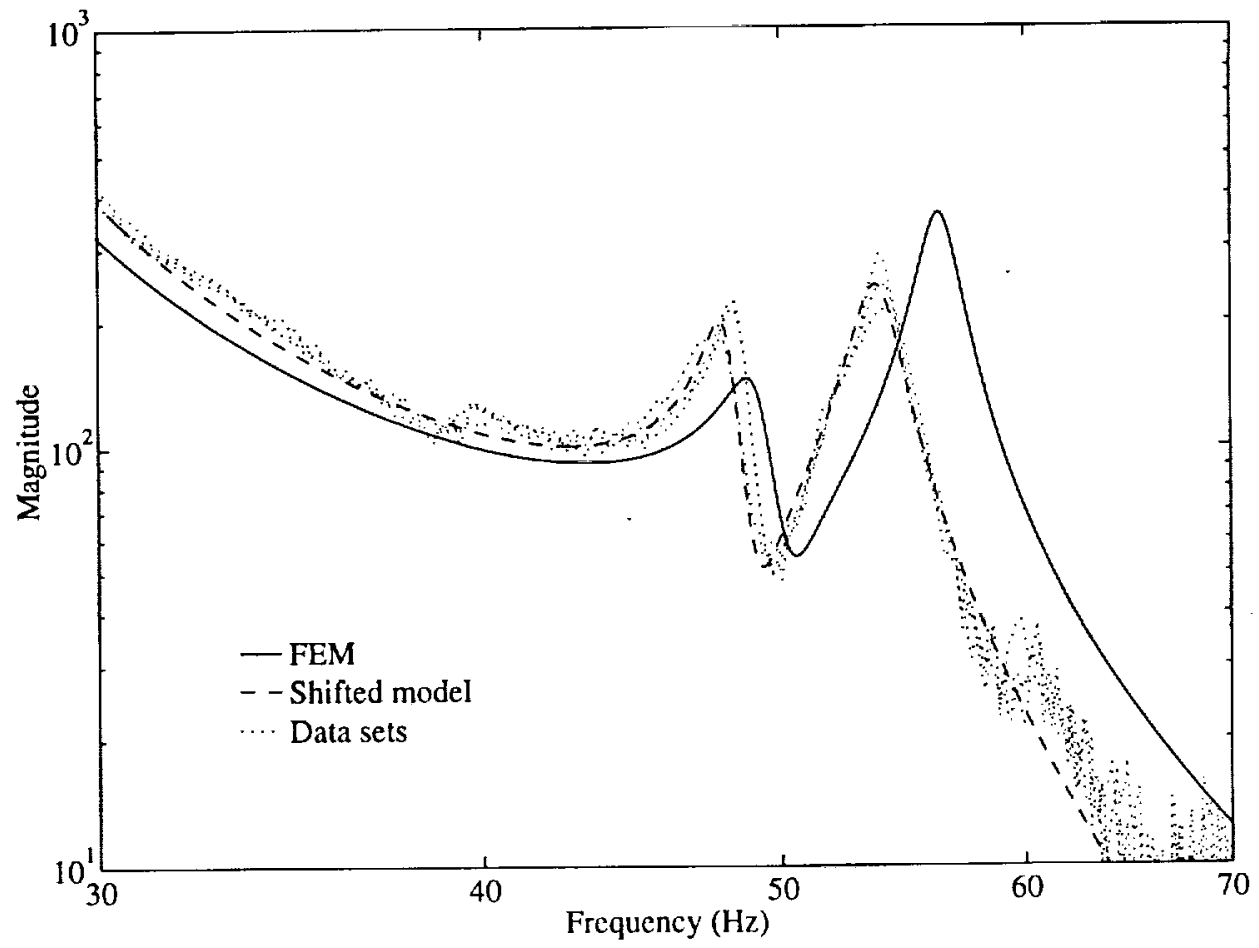

(b) Shifted model fits nicely as the "mean" model.

Figure 3.3: Development of 1-g control design and uncertainty models. 


\begin{tabular}{|c|c|c|c|c|c|c|}
\hline \multirow[b]{2}{*}{ Mode Type } & \multicolumn{3}{|c|}{ Frequency } & \multicolumn{3}{|c|}{ Damping Ratio } \\
\hline & $f_{\text {fem }}(\mathrm{Hz})$ & $\overline{\Delta f}(\%)$ & $\widehat{\Delta f}(\%)$ & $\zeta_{\text {fem }}(\%)$ & $\overline{\Delta \zeta}(\%)$ & $\widehat{\Delta \zeta}(\%)$ \\
\hline Suspension - rotary pend. & 0.24 & 0.1 & 0.3 & 9.1 & 0.1 & 0.2 \\
\hline Suspension - tilt & 0.44 & -0.2 & 1.7 & 8.0 & -4.3 & 2.9 \\
\hline Primary X Gimbal & 1.19 & 4.2 & 10.5 & 3.7 & -1.9 & 2.2 \\
\hline Primary Z Gimbal & 1.23 & 6.9 & 4.2 & 5.6 & -0.9 & 5.0 \\
\hline Secondary X Gimbal & 1.26 & 32.9 & 35.7 & 5.0 & 0.7 & 9.3 \\
\hline Secondary Z Gimbal & 1.30 & 12.6 & 2.5 & 5.9 & 1.9 & 8.7 \\
\hline Suspension - comp. pend. & 1.84 & -1.6 & 12.1 & 6.6 & -5.2 & 1.8 \\
\hline $1^{\text {st }} \mathrm{Z}$ bending & 2.24 & -1.6 & 2.3 & 2.2 & -1.6 & 0.3 \\
\hline $1^{\text {st }} \mathrm{Y}$ bending & 4.60 & -5.4 & 4.4 & 6.9 & -2.3 & 4.4 \\
\hline Suspension $-1^{\text {st }} \mathrm{X}$ violin & 7.13 & -1.0 & 1.5 & 1.4 & -0.1 & 3.4 \\
\hline Suspension $-1^{\text {st }} Z$ violin & 7.24 & -1.5 & 1.4 & 0.5 & 0.0 & 0.2 \\
\hline $2^{\text {nd }} \mathrm{Z}$ bending & 9.56 & 2.4 & 0.9 & 1.2 & 1.3 & 1.9 \\
\hline $2^{\text {nd }} Y$ bending & 9.80 & 4.2 & 1.2 & 3.9 & -3.2 & 2.9 \\
\hline $3^{\text {rd }} \mathrm{Z}$ bending & 11.04 & -1.3 & 5.6 & 3.7 & 0.3 & 5.8 \\
\hline $3^{\text {rd }} \mathrm{Y}$ bending & 11.29 & -0.3 & 8.1 & 5.9 & -4.7 & 0.9 \\
\hline $3^{\text {rd }} Y$ bending & 13.14 & 3.3 & 11.6 & 5.4 & -3.7 & 0.9 \\
\hline Suspension $-2^{\text {nd }} X$ violin & 15.12 & -1.7 & 2.1 & 2.0 & -0.5 & 1.8 \\
\hline $4^{\text {th }} \mathrm{Z}$ bending & 16.19 & 1.5 & 2.6 & 1.8 & -0.5 & 1.4 \\
\hline $4^{\text {th }} Y$ bending & 17.82 & 0.4 & 1.3 & 0.9 & -0.1 & 0.1 \\
\hline $5^{\text {th }} Y$ bending & 24.90 & 1.2 & 1.2 & 1.4 & -0.0 & 0.3 \\
\hline $5^{\text {th }} \mathrm{Z}$ bending & 39.41 & -2.4 & 3.2 & 1.4 & -0.1 & 1.0 \\
\hline $6^{\text {th }} \mathrm{Z}$ bending & 48.84 & -5.1 & 2.6 & 1.9 & -0.7 & 0.5 \\
\hline $6^{\text {th }} \mathrm{Y}$ bending & 49.01 & -2.2 & 2.9 & 1.6 & -0.7 & 0.2 \\
\hline $7^{\text {th }} \mathrm{Y}$ bending & 56.16 & -4.5 & 1.1 & 1.5 & -0.1 & 0.9 \\
\hline
\end{tabular}

Table 3.3: MACE 1-g uncertainty model.

frequency errors and bounds are less than $5 \%$. The damping errors are quite large, however. The primary reason for this is the finite element model was updated using frequency response data. The windowing and averaging in the process of creating the frequency response has the adverse effects of making the system appear more heavily damped [49]. Because the raw time histories are used in the DEKF, the damping ratios are higher, and in addition, more accurate. 


\section{Chapter 4}

\section{Uncertainty Localization and}

\section{Projection}

Once the uncertainties for the $1-\mathrm{g}$ finite element model in the form of mean error and variance uncertainties on the eigenvalues and eigenvectors is developed, the next step is to attempt to use all of the information available to predict the uncertainties in $0-\mathrm{g}$. The method proposed in this work is to localize both the mean error and variance uncertainties to specific degrees of freedom in the structure, and then project them into 0-g. This chapter first introduces the complete error localization and projection problem. Then, two techniques are described to localize the uncertainties. The first is a matrix type technique which was used for designing pre-programmed controllers for MACE on STS-67. The next method is a more practical technique which uses first order sensitivities and was derived after the MACE flight. In addition, the projection of the localized uncertainties from 1-g to $0-\mathrm{g}$ is developed for both methods. Finally, once the theoretical work has been detailed, a small order example is given to demonstrate each of these methods, and their practical limitations. 


\subsection{Problem Formulation}

The development of the uncertainty localization and projection methods is given in the most general form. Therefore, the $1-\mathrm{g}$ system is referred to as the "Nominal" system and is denoted $(\cdot)_{N}$. The $0-\mathrm{g}$ system is referred to as the "Modified" system and is denoted $(\cdot)_{M}$. This allows the uncertainty localization and projection methods to be easily developed and applied to different systems from the $1-\mathrm{g}$ to $0-\mathrm{g}$ uncertainty model development described in this work.

The dynamics for the discretized finite element model of the nominal system can be written as

$$
M_{\mathrm{N}} \ddot{\eta}_{\mathrm{N}}+C_{\mathrm{N}} \dot{\eta}_{\mathrm{N}}+K_{\mathrm{N}} \eta_{\mathrm{N}}=0
$$

where $M_{\mathrm{N}} \in \mathbb{R}^{n_{\mathrm{N}} \times n_{\mathrm{N}}}$, and $K_{\mathrm{N}} \in \mathbb{R}^{n_{\mathrm{N}} \times n_{\mathrm{N}}}$ are the mass and stiffness matrices respectively and $\eta_{\mathrm{N}}$ is a vector of $n_{\mathrm{N}}$ physical degrees of freedom. Assuming proportional damping, the dynamics can be described by solving the $n_{\mathrm{N}}$ degree of freedom, linear structural generalized eigenvalue problem

$$
-M_{\mathrm{N}} \Phi_{\mathrm{N}} \Lambda_{\mathrm{N}}+K_{\mathrm{N}} \Phi_{\mathrm{N}}=0
$$

where $\Lambda_{\mathrm{N}} \in \mathbb{R}^{n_{\mathrm{N}} \times n_{\mathrm{N}}}$ and $\Phi_{\mathrm{N}} \in \mathbb{R}^{n_{\mathrm{N}} \times n_{\mathrm{N}}}$ are the eigenvalues and eigenvectors given by

$$
\begin{aligned}
\Phi_{\mathrm{N}} & =\left[\begin{array}{lll}
\phi_{\mathrm{N} 1} & \ldots & \phi_{\mathrm{N} n_{\mathrm{N}}}
\end{array}\right] \\
\Lambda_{\mathrm{N}} & =\operatorname{diag}\left[\begin{array}{lll}
\lambda_{\mathrm{N} 1} & \ldots & \lambda_{\mathrm{N} n_{\mathrm{N}}}
\end{array}\right]
\end{aligned}
$$

The eigenvectors are assumed to be mass normalized such that

$$
\begin{aligned}
& \Phi_{\mathrm{N}}^{T} M_{\mathrm{N}} \Phi_{\mathrm{N}}=I \\
& \Phi_{\mathrm{N}}^{T} K_{\mathrm{N}} \Phi_{\mathrm{N}}=\Lambda_{\mathrm{N}}
\end{aligned}
$$

It is assumed that all of the $n_{\mathrm{N}}$ eigenvalues and eigenvectors are available. This assumption can be easily relaxed, although it is important to the techniques developed here to solve for as many modes as possible, even though the accuracy of the higher modes might be suspect. 
The finite element model is assumed to be an accurate representation of the hardware. The mean error and variance uncertainties which exist between the nominal finite element model and hardware can then be defined using a first order perturbation to the nominal system [63].

$$
\begin{aligned}
& \tilde{M}_{\mathrm{N}}=M_{\mathrm{N}}+\Delta M_{\mathrm{N}} \\
& \tilde{K}_{\mathrm{N}}=K_{\mathrm{N}}+\Delta K_{\mathrm{N}} \\
& \tilde{\Lambda}_{\mathrm{N}}=\Lambda_{\mathrm{N}}+\Delta \Lambda_{\mathrm{N}} \\
& \tilde{\Phi}_{\mathrm{N}}=\Phi_{\mathrm{N}}+\Delta \Phi_{\mathrm{N}}
\end{aligned}
$$

where $(\tilde{*})$ refers to the linear model description of the hardware (i.e. data). It is also assumed that the eigenvalue and eigenvector perturbations are only relative for the measured parameters, that is $\tilde{n}_{\mathrm{N}} \leq n_{\mathrm{N}}$ eigenvalues and the mode shapes at the $r_{\mathrm{N}} \leq n_{\mathrm{N}}$ degrees of freedom.

Under the assumption that the nominal eigenvectors $\Phi_{\mathrm{N}}$ are a sufficient set of basis vectors which describe the hardware dynamics, changes in the eigenvectors, $\Delta \Phi_{N}$, can be written as a linear combination of the original eigenvectors,

$$
\Delta \Phi_{\mathrm{N}}=\Phi_{\mathrm{N}} \Psi_{\mathrm{N}}
$$

It is this equation which requires the use of as many of the eigenvectors as possible to be retained in the finite element solution. The higher order modes, even though they might not be accurate, create a sufficient basis for representing the eigenvalue perturbations.

An analogous formulation can be developed for the modified system. The dynamics, orthogonality conditions, and perturbation equations for the modified system are

$$
\begin{gathered}
-M_{\mathrm{M}} \Phi_{\mathrm{M}} \Lambda_{\mathrm{M}}+K_{\mathrm{M}} \Phi_{\mathrm{M}}=0 \\
\Phi_{\mathrm{M}}^{T} M_{\mathrm{M}} \Phi_{\mathrm{M}}=I \\
\Phi_{\mathrm{M}}^{T} K_{\mathrm{M}} \Phi_{\mathrm{M}}=\Lambda_{\mathrm{M}}
\end{gathered}
$$




$$
\begin{gathered}
\tilde{M}_{\mathrm{M}}=M_{\mathrm{M}}+\Delta M_{\mathrm{M}} \\
\tilde{K}_{\mathrm{M}}=K_{\mathrm{M}}+\Delta K_{\mathrm{M}} \\
\tilde{\Lambda}_{\mathrm{M}}=\Lambda_{\mathrm{M}}+\Delta \Lambda_{\mathrm{M}} \\
\tilde{\Phi}_{\mathrm{M}}=\Phi_{\mathrm{M}}+\Delta \Phi_{\mathrm{M}} \\
\Delta \Phi_{\mathrm{M}}=\Phi_{\mathrm{M}} \Psi_{\mathrm{M}}
\end{gathered}
$$

where $M_{\mathrm{M}} \in \mathbb{R}^{n_{\mathrm{M}} \times_{\mathrm{M}}}, K_{\mathrm{M}} \in \mathbb{R}^{n_{\mathrm{M}} \times n_{\mathrm{M}}}, \Lambda_{\mathrm{M}} \in \mathbb{R}^{n_{\mathrm{M}} \times n_{\mathrm{M}}}$, and $\Phi_{\mathrm{M}} \in \mathbb{R}^{n_{\mathrm{M}} \times n_{\mathrm{M}}}$. Note the assumption still holds that the eigenvalues and eigenvector perturbations are only relative for the measured parameters, that is $\tilde{n}_{\mathrm{M}} \leq n_{\mathrm{M}}$ eigenvalues and the mode shapes at the $r_{\mathrm{M}} \leq n_{\mathrm{M}}$ degrees of freedom.

Figure 4.1 gives the strategy for the uncertainty localization and projection process for a general system. Given a finite element model and data sets for the nominal system, an uncertainty model is developed, as shown in the two top blocks. This uncertainty model is in the form of mean and covariance uncertainties of the eigenvalues and eigenvectors between the model and hardware, as presented in Chapter 2. Once these uncertainties have been identified, they are localized to specific physical degrees of freedom of the finite element model, in the form of mean error and variance uncertainties of the physical mass and stiffness matrices. A mapping is then created between the degrees of freedom of the nominal and modified systems. This mapping is used to map the mass and stiffness uncertainties of the nominal system into the modified system. The modified finite element model is then used to project the mapped mass and stiffness uncertainties, creating an uncertainty model for the modified system in the form of mean and covariance uncertainties of the eigenvalues and eigenvectors. This is shown in the bottom two blocks of Figure 4.1.

One of the most important aspects in the development of these methods is that the nominal uncertainty model (and subsequent uncertainty localization and projection), is based on incomplete information: the number of frequencies (eigenvalues) that are measured $\left(\tilde{n}_{\mathrm{N}}\right)$ is less than (or equal to) the total number of modes in the system, or $\tilde{n}_{\mathrm{N}} \leq n_{\mathrm{N}}$; the number of distinct actuators and sensors (mode shapes) that are 


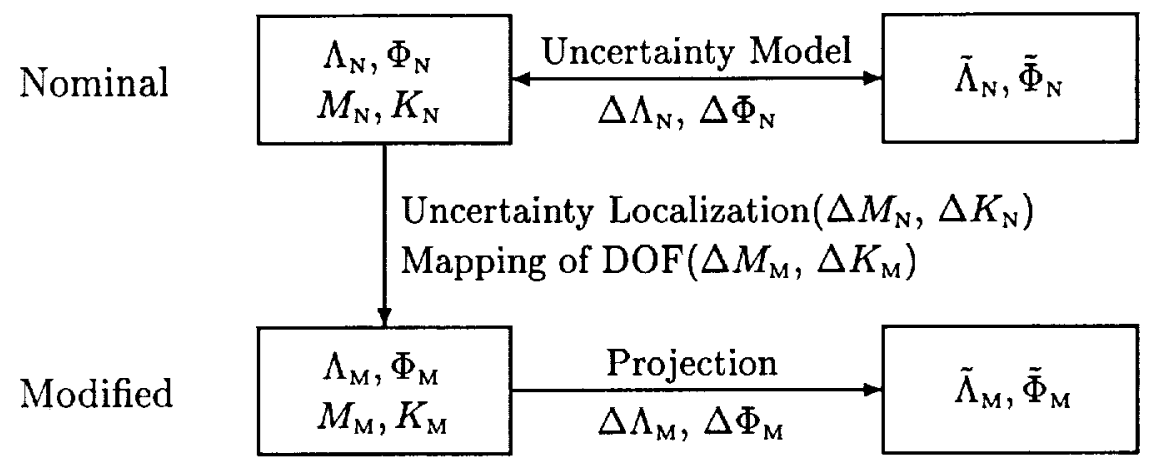

Figure 4.1: Uncertainty model development problem.

measured $\left(r_{\mathrm{N}}\right)$ is less (or equal to) the number of original degrees of freedom, or $r_{\mathrm{N}} \leq n_{\mathrm{N}}$. This constraint ensures the methods developed are the most practical.

With the groundwork of the uncertainty localization and projection problems laid out as in Figure 4.1, two methods are introduced. The first is a matrix method which manipulates the perturbed matrices introduced in this section to give mean error and variance uncertainties for the modified system in terms of the mean error and variance uncertainties for the nominal system. The second method is a sensitivity method where a specific structure is given to the mass and stiffness error matrices in the form of macroelements and scaling factors.

\subsection{Matrix Method}

The first method presented that localizes nominal uncertainties to specific degrees of freedom and projects them into a modified environment is called the matrix method, and was used in the design of controllers for the MACE flight. This method is also detailed in Ref. 48. The matrix method attempts to find the mean error and variance uncertainties for the modified system by directly manipulating the matrix perturbations given in the previous section. Although similar in name to other matrix type localization methods $[16,15]$, this method finds a closed form solution for both mass and stiffness errors based on the given measurements. 


\subsubsection{Uncertainty Localization}

The first step in developing the matrix method is uncertainty localization. The orthogonality conditions for true dynamics of the nominal hardware are written as

$$
\begin{aligned}
& \tilde{\Phi}_{\mathrm{N}}^{T} \tilde{M}_{\mathrm{N}} \tilde{\Phi}_{\mathrm{N}}=I \\
& \tilde{\Phi}_{\mathrm{N}}^{T} \tilde{K}_{\mathrm{N}} \tilde{\Phi}_{\mathrm{N}}=\tilde{\Lambda}_{\mathrm{N}}
\end{aligned}
$$

Enforcing these conditions, and substituting the perturbed dynamics of Equations 4.5 and 4.6 into Equation 4.11 and ignoring higher order terms, yields

$$
\begin{aligned}
& \Phi_{\mathrm{N}}^{T} \Delta M_{\mathrm{N}} \Phi_{\mathrm{N}}=-\Psi_{\mathrm{N}}-\Psi_{\mathrm{N}}^{T} \\
& \Phi_{\mathrm{N}}^{T} \Delta K_{\mathrm{N}} \Phi_{\mathrm{N}}=\Delta \Lambda_{\mathrm{N}}-\Lambda_{\mathrm{N}} \Psi_{\mathrm{N}}-\Psi_{\mathrm{N}}^{T} \Lambda_{\mathrm{N}}
\end{aligned}
$$

The known errors $\Delta \Lambda_{\mathrm{N}}, \Psi_{\mathrm{N}}$ can then be translated into physical mass and stiffness errors by employing a pseudoinverse,

$$
\begin{aligned}
\Delta M_{\mathrm{N}} & =\left(\Phi_{\mathrm{N}}^{T}\right)^{+}\left[-\Psi_{\mathrm{N}}-\Psi_{\mathrm{N}}^{T}\right]\left(\Phi_{\mathrm{N}}\right)^{+} \\
\Delta K_{\mathrm{N}} & =\left(\Phi_{\mathrm{N}}^{T}\right)^{+}\left[\Delta \Lambda_{\mathrm{N}}-\Lambda_{\mathrm{N}} \Psi_{\mathrm{N}}-\Psi_{\mathrm{N}}^{T} \Lambda_{\mathrm{N}}\right]\left(\Phi_{\mathrm{N}}\right)^{+}
\end{aligned}
$$

where $(\cdot)^{+}$refers to a pseudoinverse. A pseudoinverse is employed as a result of the above equations being underdetermined, or the measured modes and degrees of freedom are less than (or equal to) that of the total system, $\tilde{n}_{\mathrm{N}} \leq n_{\mathrm{N}}$ and $r_{\mathrm{N}} \leq n_{\mathrm{N}}$. Therefore, although the optimization problem has been solved correctly, the solution that is found may not be physically realizable.

Because the errors are now in physical mass and stiffness form, they correspond to particular degrees of freedom. These physical errors of the nominal system can then be mapped to a modified system. For instance, suppose there is a truss structure with a plate at one end, and there is a mass error of $5 \%$ corresponding to this degree of freedom. If the same plate is also in the modified structure, the mass error of that degree of freedom in the modified structure is also $5 \%$. Under this assumption, the mapping of degrees of freedom is denoted $T_{\mathrm{MN}}$, or

$$
\eta_{\mathrm{M}}=T_{\mathrm{MN}} \eta_{\mathrm{N}}
$$


The physical mass and stiffness errors of the modified system can then be written in terms of the nominal mass and stiffness errors

$$
\begin{aligned}
\Delta M_{\mathrm{M}} & =T_{\mathrm{MN}} \Delta M_{\mathrm{N}} T_{\mathrm{MN}}^{T} \\
\Delta K_{\mathrm{M}} & =T_{\mathrm{MN}} \Delta K_{\mathrm{N}} T_{\mathrm{MN}}^{T}
\end{aligned}
$$

This assumes, however, that the mapping of degrees of freedom between the two systems can separate the nominal and modified errors. For a few cases, most notably the modeling of gravity effects in a flexible system, this cannot be done. The modeling of gravity stiffening and initial deformations are modeled as a superpose onto the finite element stiffness matrix. The matrix method, therefore, cannot differentiate between errors in modeling the physical test article, and errors in modeling the gravity effects.

\subsubsection{Uncertainty Projection}

Once the physical errors of the modified system are known, the eigenvalue and eigenvector errors can be found by manipulating the modified system. The linear structural eigenvalue problem for the true dynamics of the modified hardware can be written as

$$
-\tilde{M}_{\mathrm{M}} \tilde{\phi}_{\mathrm{M} i} \tilde{\lambda}_{\mathrm{M} i}+\tilde{K}_{\mathrm{M}} \tilde{\phi}_{\mathrm{M} i}=0
$$

Substituting Equations 4.9-4.10 into Equation 4.7, and premultiplying by $\phi_{\mathrm{M} i}^{T}$ and $\phi_{\mathrm{M} j}^{T}$, yields

$$
\begin{aligned}
\Delta \lambda_{\mathrm{M} i} & =\phi_{\mathrm{M} i}^{T}\left[\Delta K_{\mathrm{M}}-\lambda_{\mathrm{M} i} \Delta M_{\mathrm{M}}\right] \phi_{\mathrm{M} i} \\
{\left[\lambda_{\mathrm{M} i}-\lambda_{\mathrm{M} j}\right] \psi_{\mathrm{M} j i} } & =\phi_{\mathrm{M} j}^{T}\left[\Delta K_{\mathrm{M}}-\lambda_{\mathrm{M} j} \Delta M_{\mathrm{M}}\right] \phi_{\mathrm{M} i}
\end{aligned}
$$

Using Kronecker algebra [64], the eigenvalue errors and the matrix $\Psi$ that defines the eigenvector errors of the modified system are found to be linear in terms of known nominal errors, $\Delta \Lambda_{\mathrm{N}}, \Delta \Phi_{\mathrm{N}}$.

$$
\begin{gathered}
\Delta \lambda_{\mathrm{Mi}}=\bar{C}_{i}\left[\begin{array}{l}
\operatorname{vec}\left\{\lambda_{\mathrm{N} i}\right\} \\
\operatorname{vec} \Delta \Phi_{\mathrm{N}}
\end{array}\right] \\
\psi_{\mathrm{M} j i}=\bar{D}_{j i}\left[\begin{array}{l}
\operatorname{vec}\left\{\lambda_{\mathrm{N} i}\right\} \\
\operatorname{vec} \Delta \Phi_{\mathrm{N}}
\end{array}\right]
\end{gathered}
$$


where

$$
\begin{aligned}
& \bar{C}_{i}=\left[\begin{array}{ll}
\bar{A}_{i}{ }^{T} \otimes \bar{A}_{i}{ }^{T} U_{\lambda} & \left(\bar{A}_{i}{ }^{T} \otimes \bar{A}_{i}{ }^{T} \bar{B}_{i}+\bar{A}_{i}^{T} \bar{B}_{i} \otimes \bar{A}_{i}^{T} U_{\phi}\right) \cdot \Phi_{\mathrm{N}}^{-1}
\end{array}\right]
\end{aligned}
$$

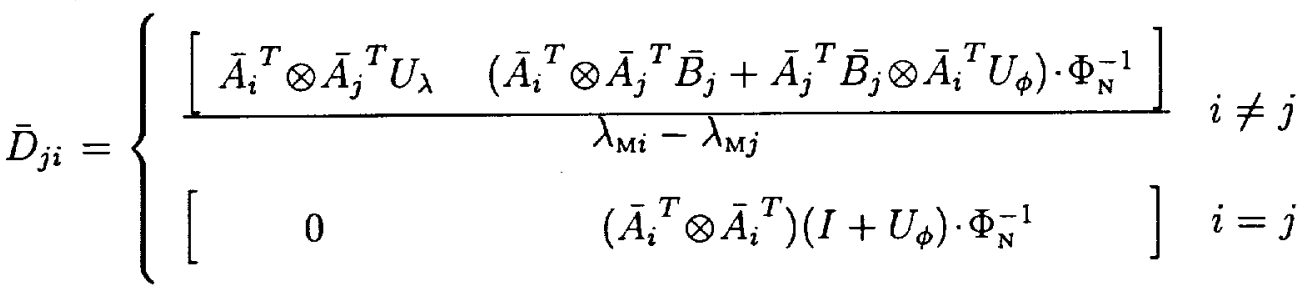

$$
\begin{aligned}
& \bar{A}_{i}=\left(\Phi_{\mathrm{N}}\right)^{+} T_{\mathrm{MN}}^{T} \phi_{\mathrm{M} i}, \quad \operatorname{vec} \Delta \Phi_{\mathrm{N}}^{T}=U_{\phi} \operatorname{vec} \Delta \Phi_{\mathrm{N}} \\
& \bar{B}_{i}=-\Lambda_{\mathrm{N}}-\lambda_{\mathrm{M} i} I, \quad \operatorname{vec} \Delta \Lambda_{\mathrm{N}}=U_{\lambda} \operatorname{vec}\left\{\lambda_{\mathrm{N} i}\right\}
\end{aligned}
$$

The unknown eigenvalue and eigenvector errors for the modified system are then shown to be linear in terms of the known errors from the nominal system. Defining

$$
F_{i}=\left[\begin{array}{ll}
\bar{C}_{i} & \Phi_{N} \bar{D}_{i}
\end{array}\right]
$$

the modified errors are

$$
\left[\begin{array}{c}
\Delta \lambda_{\mathrm{M} i} \\
\Delta \phi_{\mathrm{M} i}
\end{array}\right]=F_{i}\left[\begin{array}{l}
\operatorname{vec}\left\{\lambda_{\mathrm{N} i}\right\} \\
\operatorname{vec} \Delta \Phi_{\mathrm{N}}
\end{array}\right]
$$

Because of the linearity, the expected mean and covariance can easily be calculated.

$$
\begin{aligned}
\mathrm{E}\left[\begin{array}{l}
\Delta \lambda_{\mathrm{M} i} \\
\Delta \phi_{\mathrm{M} i}
\end{array}\right] & =F_{i} \mathrm{E}\left[\begin{array}{l}
\operatorname{vec}\left\{\lambda_{\mathrm{N} i}\right\} \\
\operatorname{vec} \Delta \Phi_{\mathrm{N}}
\end{array}\right] \\
\operatorname{Cov}\left[\begin{array}{l}
\Delta \lambda_{\mathrm{M} i} \\
\Delta \phi_{\mathrm{M} i}
\end{array}\right] & =F_{i} \operatorname{Cov}\left[\begin{array}{c}
\operatorname{vec}\left\{\lambda_{\mathrm{N} i}\right\} \\
\operatorname{vec} \Delta \Phi_{\mathrm{N}}
\end{array}\right] F_{i}^{T}
\end{aligned}
$$

where $\mathrm{E}[\cdot]$ and $\operatorname{Cov}[\cdot]$ denote the expectation and covariance operators respectively.

The mean errors and bounds for the relevant parameters of the modified system, i.e. the frequencies and eigenvectors, can then computed from Equations 4.24 and 4.25. The eigenvector mean errors and bounds are computed directly,

$$
\begin{aligned}
& \overline{\Delta \phi}_{\mathrm{Mij}}=\mathrm{E}\left[\Delta \phi_{\mathrm{M} i j}\right] \\
& \widehat{\Delta \phi}_{\mathrm{M} i j}=3 \cdot \sqrt{\operatorname{var}\left[\Delta \phi_{\mathrm{M} i j}\right]}
\end{aligned}
$$

where $\operatorname{var}[\cdot]$ denotes the variance operator, which is the diagonal of the covariance matrix in Equation 4.25. The mean errors for the eigenvalues can be found similarly. 
However, more meaningful uncertainty bounds for control design are bounds on fre-

quencies rather than eigenvalues of the modified system. Assuming the eigenvalue error is small compared to the eigenvalue, the frequency perturbation can be written using a first order Taylor expansion.

$$
\Delta f_{\mathrm{M} i}=\frac{\Delta \lambda_{\mathrm{M} i}}{2 f_{\mathrm{M} i}}
$$

Using this equation, the mean errors and bounds for each sample frequency can be found.

$$
\begin{aligned}
\overline{\Delta f}_{\mathrm{M} i} & =\frac{\mathrm{E}\left[\Delta \lambda_{\mathrm{M} i}\right]}{2 f_{\mathrm{M}}} \\
\widehat{\Delta f}_{\mathrm{M} i} & =\frac{3 \cdot \sqrt{\operatorname{var}\left[\Delta \lambda_{\mathrm{M} i}\right]}}{2 f_{\mathrm{M} i}}
\end{aligned}
$$

\subsection{Sensitivity Method}

The second method used for uncertainty localization and projection is called the sensitivity method. The use of first order sensitivities for model updating can be traced back three decades $[31,23]$. The method described here, however, develops a complete uncertainty localization strategy that not only uses the first order sensitivities as described in Refs. 31,23 , but also addresses problems such as systematically choosing the update parameters, and handling an insufficient number of modal measurements. In addition, the technique is adapted for the localization of variance uncertainties, as well as mean errors. The localized mean error and variance uncertainties using the sensitivity method are also projected to find the modified uncertainty model.

\subsubsection{Uncertainty Localization}

The linear structural eigenvalue problem for the true dynamics of the nominal hardware can be written as

$$
-\tilde{M}_{\mathrm{N}} \tilde{\phi}_{\mathrm{N} i} \tilde{\lambda}_{\mathrm{N} i}+\tilde{K}_{\mathrm{N}} \tilde{\phi}_{\mathrm{N} i}=0
$$


Substituting Equations 4.5-4.6 into Equation 4.1, and premultiplying by $\phi_{\mathrm{N} i}^{T}$ and $\phi_{\mathrm{N} j}^{T}$, yields

$$
\begin{aligned}
\Delta \lambda_{\mathrm{N} i} & =\phi_{\mathrm{N} i}^{T}\left[\Delta K_{\mathrm{N}}-\lambda_{\mathrm{N} i} \Delta M_{\mathrm{N}}\right] \phi_{\mathrm{N} i} \\
{\left[\lambda_{\mathrm{N} i}-\lambda_{\mathrm{N} j}\right] \psi_{\mathrm{N} j i} } & =\phi_{\mathrm{N} j}^{T}\left[\Delta K_{\mathrm{N}}-\lambda_{\mathrm{N} j} \Delta M_{\mathrm{N}}\right] \phi_{\mathrm{N} i}
\end{aligned}
$$

Instead of manipulating the matrices as was done for the matrix method, a particular form is supplied for the mass and stiffness error matrices. These perturbations are written as

$$
\begin{aligned}
\Delta K_{\mathrm{N}} & =\sum_{j=1}^{k_{\mathrm{N}}} \alpha_{j} K_{j} \\
\Delta M_{\mathrm{N}} & =\sum_{j=1}^{m_{\mathrm{N}}} \beta_{j} M_{j}
\end{aligned}
$$

where $K_{j}$ and $M_{j}$ are termed macroelements, $\alpha_{j}$ and $\beta_{j}$ are macroelemental scale factors which scale the relative size of each macroelement, and $k_{\mathrm{N}}$ and $m_{\mathrm{N}}$ refer to the total number of macroelements for the nominal system. These macroelements can be in the form of a finite element, a part of an element, or groups of elements. A more detailed discussion of these macroelements is given subsequently.

Substituting Equations 4.32 into Equations 4.30-4.31 and rearranging, the following form results for the eigenvalue and eigenvector errors.

$$
\begin{aligned}
\Delta \lambda_{\mathrm{N} i} & =\sum_{j=1}^{k_{\mathrm{N}}}\left[\phi_{\mathrm{N} i}^{T} K_{j} \phi_{\mathrm{N} i}\right] \alpha_{j}-\lambda_{\mathrm{N} i} \sum_{j=1}^{m_{\mathrm{N}}}\left[\phi_{\mathrm{N} i}^{T} M_{j} \phi_{\mathrm{N} i}\right] \beta_{j} \\
\Delta \phi_{\mathrm{N} i} & =\sum_{j=1}^{k_{\mathrm{N}}}\left[\sum_{\substack{k=1 \\
k \neq i}}^{n_{\mathrm{N}}} \frac{\phi_{\mathrm{N} i}^{T} K_{j} \phi_{\mathrm{N} k}}{\lambda_{\mathrm{N} k}-\lambda_{\mathrm{N} i}} \phi_{\mathrm{N} k}\right] \alpha_{j}-\sum_{j=1}^{m_{\mathrm{N}}}\left[\sum_{\substack{k=1 \\
k \neq i}}^{n_{\mathrm{N}}} \lambda_{\mathrm{N} k} \frac{\phi_{\mathrm{N} i}^{T} M_{j} \phi_{\mathrm{N} k}}{\lambda_{\mathrm{N} k}-\lambda_{\mathrm{N} i}} \phi_{\mathrm{N} k}-\frac{\phi_{\mathrm{N} i}^{T} M_{j} \phi_{\mathrm{N} i}}{2} \phi_{\mathrm{N} i}\right] \beta_{j}
\end{aligned}
$$

The above equations are linear in terms of the macroelemental scale factors $\alpha_{j}$ and $\beta_{j}$. Therefore, this equation can then be written in the form

$$
\left[\begin{array}{c}
\Delta \lambda_{\mathrm{N} i} \\
\Delta \phi_{\mathrm{N} i}
\end{array}\right]=S_{\mathrm{N} i}\left[\begin{array}{l}
\alpha_{\mathrm{N}} \\
\beta_{\mathrm{N}}
\end{array}\right]
$$


where

$$
\begin{aligned}
& S_{\mathrm{N} i 11}=\left[\begin{array}{lll}
\phi_{\mathrm{N} i}^{T} K_{1} \phi_{\mathrm{N} i} & \cdots & \phi_{\mathrm{N} i}^{T} K_{k_{\mathrm{N}}} \phi_{\mathrm{N} i}
\end{array}\right] \\
& S_{\mathrm{N} i 12}=\left[\begin{array}{llll}
-\lambda_{\mathrm{N} i} \phi_{\mathrm{N} i}^{T} M_{1} \phi_{\mathrm{N} i} & \cdots & -\lambda_{\mathrm{N} i} \phi_{\mathrm{N} i}^{T} K_{k_{\mathrm{N}}} \phi_{\mathrm{N} i}
\end{array}\right] \\
& S_{\mathrm{N} i 21}=\left[\begin{array}{lll}
\sum_{\substack{k=1 \\
k \neq i}}^{n_{N}} \frac{\phi_{N i}^{T} K_{1} \phi_{N k}}{\lambda_{N k}-\lambda_{N i}} \phi_{\mathrm{N} k} & \cdots \sum_{\substack{k=1 \\
k \neq i}}^{n_{N}} \frac{\phi_{N i}^{T} K_{k} \phi_{N k}}{\lambda_{N k}-\lambda_{N i}} \phi_{N k}
\end{array}\right]
\end{aligned}
$$

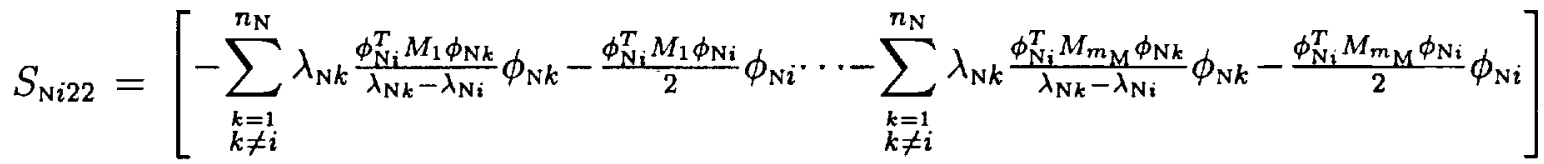

A set of linear equations can be developed for all measured errors, giving

$$
\left[\begin{array}{c}
\operatorname{vec}\left\{\Delta \lambda_{\mathrm{N} i}\right\} \\
\operatorname{vec} \Delta \phi_{\mathrm{N} i}
\end{array}\right]=S_{\mathrm{N}}\left[\begin{array}{c}
\alpha_{\mathrm{N}} \\
\beta_{\mathrm{N}}
\end{array}\right]
$$

The matrix $S_{\mathrm{N}}$ is a called a sensitivity matrix. Note that there are $\tilde{n}_{\mathrm{N}} \leq n_{\mathrm{N}}$ measured eigenvalues and $r_{\mathrm{N}} \leq n_{\mathrm{N}}$ measurement locations.

In order to calculate the macroelemental scale factors, and subsequently the mass and stiffness errors, a pseudoinverse is again employed. If the choice of macroelements and scale factors is made such that their number is less than or equal to the number of measured errors, or

$$
k_{\mathrm{N}}+m_{\mathrm{N}} \leq\left(r_{\mathrm{N}}+1\right) \cdot \tilde{n}_{\mathrm{N}}
$$

then Equation 4.36 is overdetermined, rather than underdetermined as it was for the matrix method (Equations 4.13 and 4.14). Therefore, the solution that results from using this pseudoinverse is physically realizable, in addition to being mathematically optimal.

A weighted pseudoinverse is then used to calculate the macroelemental scale factors

$$
\left[\begin{array}{c}
\alpha_{\mathrm{N}} \\
\beta_{\mathrm{N}}
\end{array}\right]=\left[W_{\mathrm{N}} S_{\mathrm{N}}\right]^{+} W_{\mathrm{N}}\left[\begin{array}{c}
\operatorname{vec}\left\{\Delta \lambda_{\mathrm{N} i}\right\} \\
\operatorname{vec} \Delta \phi_{\mathrm{N} i}
\end{array}\right]
$$

where the $[\cdot]^{+}$refers to the pseudoinverse and $W_{\mathrm{N}}$ is a diagonal weighting matrix

$$
W_{\mathrm{N}}=\operatorname{diag}\left\{\begin{array}{c}
\frac{c_{\lambda \mathrm{N} i}}{\left\|\lambda_{\mathrm{N} i}\right\|} \\
\frac{c_{\phi \mathrm{N} i}}{\left\|\phi_{\mathrm{N} i}\right\|}
\end{array}\right\}
$$


and $c_{(\cdot)}$ are confidence factors that are chosen is a somewhat ad hoc manner. For instance, eigenvalue uncertainties are usually easier to calculate than mode shape uncertainties, and are not as prone to measurement errors. Therefore, the confidence factors are chosen to be $c_{\lambda \mathrm{N} i}=1.0$ and $c_{\phi \mathrm{N} i}=0.1$ reflecting the higher confidence in the eigenvalue measurements.

Because the macroelemental scale factors are linear in terms of the known errors, the expected value and covariance can easily be calculated

$$
\begin{aligned}
& \mathrm{E}\left[\begin{array}{c}
\alpha_{\mathrm{N}} \\
\beta_{\mathrm{N}}
\end{array}\right]=\left[\left[W_{\mathrm{N}} S_{\mathrm{N}}\right]^{+} W_{\mathrm{N}}\right] \mathrm{E}\left[\begin{array}{c}
\operatorname{vec}\left\{\Delta \lambda_{\mathrm{N} i}\right\} \\
\operatorname{vec} \Delta \phi_{\mathrm{N} i}
\end{array}\right] \\
& \operatorname{Cov}\left[\begin{array}{c}
\alpha_{\mathrm{N}} \\
\beta_{\mathrm{N}}
\end{array}\right]=\left[\left[W_{\mathrm{N}} S_{\mathrm{N}}\right]^{+} W_{\mathrm{N}}\right] \operatorname{Cov}\left[\begin{array}{c}
\operatorname{vec}\left\{\Delta \lambda_{\mathrm{N} i}\right\} \\
\operatorname{vec} \Delta \phi_{\mathrm{N} i}
\end{array}\right]\left[\left[W_{\mathrm{N}} S_{\mathrm{N}}\right]^{+} W_{\mathrm{N}}\right]^{T}
\end{aligned}
$$

\subsubsection{Uncertainty Projection}

Once in the physical domain, the mass and stiffness errors can be mapped from the nominal to the modified system. Using the general mapping of degrees of freedom between the two systems given in Equation 4.15, the mass and stiffness errors for the modified system are given by

$$
\begin{aligned}
\Delta K_{\mathrm{M}} & =\sum_{j=1}^{k_{\mathrm{M}}} \alpha_{j} T_{\mathrm{MN}} K_{j} T_{\mathrm{MN}}^{T} \\
\Delta M_{\mathrm{M}} & =\sum_{j=1}^{m_{\mathrm{M}}} \beta_{j} T_{\mathrm{MN}} M_{j} T_{\mathrm{MN}}^{T}
\end{aligned}
$$

Because the sensitivity method defines possible modeling error sources in the original derivation of the method in the form of macroelements, it can handle the superposition of errors between the nominal and modified systems. By choosing different macroelements $K_{j}$ and $M_{j}$, errors that superpose such as errors in the modeling of the test article and errors in the modeling of gravity effects, the physically correct errors can be identified. This ability is an improvement over the more constraining matrix method (Equation 4.16).

The number of macroelements for the modified system is generally less that the number for the nominal system, or $k_{\mathrm{M}} \leq k_{\mathrm{N}}$ and $m_{\mathrm{M}} \leq m_{\mathrm{N}}$. The macroelemental 
scale factors (and therefore macroelements) for the modified system can be found by ordering the nominal scale factors such that

$$
\begin{aligned}
& {\left[\alpha_{N}\right]=\left[\begin{array}{c}
\alpha_{M} \\
\vdots
\end{array}\right]} \\
& {\left[\beta_{N}\right]=\left[\begin{array}{c}
\beta_{M} \\
\vdots
\end{array}\right]}
\end{aligned}
$$

By selecting only those macroelements (and scale factors) that affect the modified system and mapping those macroelements, the mass and stiffness errors for the modified system can be found using Equation 4.41.

The next step is to find the eigenvalue and eigenvector uncertainties in terms of the mass and stiffness uncertainties for the modified system. An analogous derivation of the sensitivity equation, given in Equation 4.33-4.36 for the nominal system, can be made for the modified system using the macroelements in Equation 4.41. This again gives a set of linear equations between the unknown eigenvalue and eigenvector errors and calculated scale factors.

$$
\left[\begin{array}{c}
\operatorname{vec}\left\{\Delta \lambda_{\mathrm{M} i}\right\} \\
\operatorname{vec} \Delta \phi_{\mathrm{M} i}
\end{array}\right]=S_{\mathrm{M}}\left[\begin{array}{l}
\alpha_{\mathrm{M}} \\
\beta_{\mathrm{M}}
\end{array}\right]
$$

where $S_{\mathrm{M}}$ is the sensitivity matrix. The expected mean and covariance are then calculated quite easily.

$$
\begin{aligned}
\mathrm{E}\left[\begin{array}{c}
\operatorname{vec}\left\{\Delta \lambda_{\mathrm{M} i}\right\} \\
\operatorname{vec} \Delta \phi_{\mathrm{M} i}
\end{array}\right] & =S_{\mathrm{M}} \mathrm{E}\left[\begin{array}{l}
\alpha_{\mathrm{M}} \\
\beta_{\mathrm{M}}
\end{array}\right] \\
\operatorname{Cov}\left[\begin{array}{c}
\operatorname{vec}\left\{\Delta \lambda_{\mathrm{M} i}\right\} \\
\operatorname{vec} \Delta \phi_{\mathrm{M} i}
\end{array}\right] & =S_{\mathrm{M}} \operatorname{Cov}\left[\begin{array}{l}
\alpha_{\mathrm{M}} \\
\beta_{\mathrm{M}}
\end{array}\right] S_{\mathrm{M}}^{T}
\end{aligned}
$$

The mean and covariance of the eigenvalues and eigenvectors can then be used to create the modified uncertainty model using Equations 4.26-4.28.

\subsubsection{Macroelements}

Macroelements are user defined matrices which should span the space of all possible modeling errors. Therefore, there are many types of macroelements from which to 
Macroelement: groups of elements

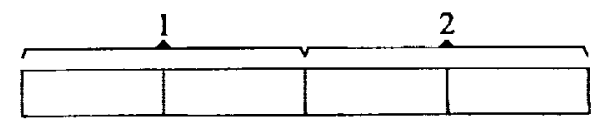

Macroelement: elements

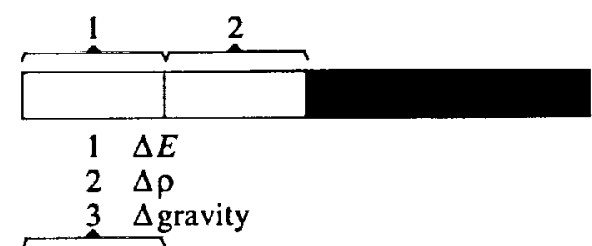

Macroelement: sub-elements

Figure 4.2: Three distinct macroelements.

choose when attempting the sensitivity method. Factors which influence the choices include: the number and location of the measurements; the number and type of modes; the types of uncertainties that may exist in the model; and the size of the finite element model. The factor which influences the choice of macroelements the most, however, is the number of unknown scale factors (macroelements) must be less than or equal to the number of measured errors, as shown in Equation 4.37.

The number of measured errors places a fundamental limit on the number of macroelements. Because of this, three distinct types of macroelements are defined: sub-elements, elements, and groups of elements. Sub-element based macroelements are those errors which lie within a particular finite element, as shown in Table 2.4. Element based macroelements are those errors which lie in different finite elements. And finally, groups of elements based macroelements are those errors which lie in different areas of the structure, as defined by different groups of elements. These different types of macroelements are shown in Figure 4.2. In this section, each type of macroelement is fully described, while in the following section, a strategy for choosing the required type of macroelements for uncertainty localization is presented.

The first choice of macroelements is based on errors within the finite element, or at the sub-element level. In Chapter 2, a thorough examination of finite element model uncertainties is presented. Physical uncertainties such as material and geometric properties are easily represented as errors within the element, as given in Table 2.4. Errors in internal couplings such as joints/attachments and actuator/sensor dynamics 
that are not in the finite element model are localized using the identification procedure such as the DEKF. Gravity effects such as geometric stiffening and initial deformations are additions to the finite element stiffness matrix. Therefore, macroelements can be developed using Equation 2.5 and Equations 2.7 and 2.8. The final two categories of uncertainties are testing and nonlinearities. Because these uncertainties cannot be expressed using linear macroelements, it is assumed that they can manifest themselves as mean error and variance uncertainties in the macroelements developed previously. This approach is a bit ad hoc, and a more thorough examination of these effects should be undertaken.

The second type of macroelement is at the element level. A single macroelement can be formed which is the summation of all of the sub-element errors corresponding to that particular finite element. This single macroelement can then be used to localize errors to that element (and other elements), even if the sub-element errors are distinct.

The final type of macroelement is grouping finite element errors themselves. When creating a finite element model, a finer and finer mesh of elements may be needed to capture the dynamics of the structure. As a result, all of the degrees of freedom are usually not experimentally measured. It is therefore occasionally useful to group finite element errors together into one macroelement. The easiest manner in which to group element errors is using insights from the finite element modeling process. For instance, if an element is split into two equivalent elements to obtain a finer mesh, errors in these two finite elements can be grouped together into one macroelement for the localization process.

In addition to using modeling insight to group element errors into macroelements, it is also useful to obtain a grouping procedure which is a function of the problem posed. For instance, if there is a section of the structure that does not have sensors, it may be difficult to localize errors to specific degrees of freedom. Taking a term from the control systems community, the element errors may be unobservable. Mathematically, these unobservable element errors manifest themselves as singularities in the sensitivity matrix. 
In order to examine this problem, the singular value decomposition of the weighted sensitivity matrix of the nominal system is written as

$$
W_{\mathrm{N}} S_{\mathrm{N}}=U_{\mathrm{N}} \Sigma_{\mathrm{N}} V_{\mathrm{N}}^{H}
$$

where $U_{\mathrm{N}}$ and $V_{\mathrm{N}}$ are left and right unitary matrices, and $\Sigma_{\mathrm{N}}$ is a matrix of singular values. If there are element errors that are unobservable, they manifest themselves as singularities, or small singular values in the decomposition. For instance, if a singular value is 0 , this is a reduction in the rank of the sensitivity matrix. For those singular values that are small, the corresponding column of the $V_{\mathrm{N}}$ matrix yields a null vector. Therefore, the unitary matrix $V_{\mathrm{N}}$ gives a guide to grouping elements. The singular value decomposition of the following matrix shows a simple example of this procedure.

$$
\left[\begin{array}{lll}
1 & 1 & 2 \\
2 & 1 & 4 \\
3 & 1 & 6 \\
4 & 1 & 8
\end{array}\right]=U\left[\begin{array}{ccc}
12.38 & 0 & 0 \\
0 & 0.81 & 0 \\
0 & 0 & 0.00 \\
0 & 0 & 0
\end{array}\right]\left[\begin{array}{ccc}
0.44 & -0.06 & 0.88 \\
0.14 & 0.98 & 0.00 \\
0.88 & -0.13 & -0.44
\end{array}\right]
$$

Notice that the original matrix has two dependent columns, i.e. the third column is two times the first. The third entry in the singular value matrix is zero, and the third column of the unitary matrix $V_{\mathrm{N}}$ shows the first and third columns are related by a factor of two. This method can be used to group elements as needed, thereby reducing the number of columns of the sensitivity matrix (which is equal to the number of macroelements) to a number which is less than the number of measured errors. This ensures the use of an overdetermined pseudoinverse, and a physically realizable structure of the solution.

By describing the unobservable element modeling errors in a mathematical manner, a different and interesting application of this method becomes apparent. By examining the rank of the sensitivity matrix, the physical locations of the sensors can be chosen such that modeling errors are more observable. This is an attempt to use the sensitivity method essentially in reverse, in order to create a better experimental setup. This interesting application is left for future work. 


\subsection{Practical Algorithm}

Too many times have there been methods developed which work quite well for simple problems, but the practical application of such methods is severely lacking. This is exemplified in the error localization literature where most of the algorithms assume the measurement of (or expansion to) all degrees of freedom. Although interesting, practical application of these methods is not realistic. This section describes the application of uncertainty localization and projection methods to a practical experiment, specifically addressing issues such as the type of uncertainty localization technique, the choices of macroelements, the pseudoinverse method, and effects of large modeling errors.

The primary item for a practical uncertainty localization and projection strategy is the choice of technique: matrix method versus sensitivity method. It has become obvious in the derivation of both methods that the sensitivity method is the most practical. This is because the pseudoinverse employed in the sensitivity method is on an overdetermined system, thus yielding physically realizable solutions. In the matrix method, the pseudoinverse is on an underdetermined system, thus yielding only a mathematical result which may not be physically realizable. Secondly, the choice of macroelements allows the user to define errors which may superpose each other. This is especially useful when gravity effects are prevalent, because they are modeled as an superposition of the finite element model stiffness matrix.

The next step in creating a practical tool is the choice of macroelements. This choice is heavily influenced by the number of errors measured. The macroelements must be selected such that the number of macroelements is larger than the number of known uncertainties. (i.e. to ensure an overdetermined pseudoinverse). The method proposed is a three step layered approach, as shown in Figure 4.3.

The uncertainties are first localized to particular areas of the structure, using the largest macroelements based on groups of elements. Once localized using the sensitivity method, the number of macroelements are reduced by throwing out those macroelements which do not contain large modeling errors. This is shown in Fig- 
1. - Macroelement: groups of elements

- Localize

- Reduce number of macroelements

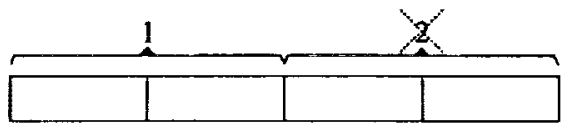

2. - Macroelement: elements

- Localize

- Reduce number of macroelements

3. - Macroelement: sub-elements

- Localize

- Reduce number of macroelements

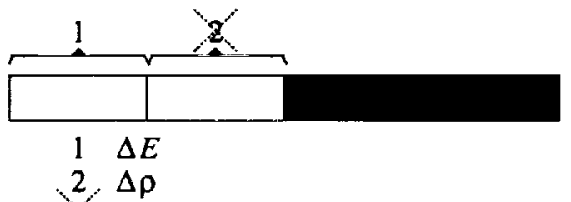

$3 \Delta$ gravity

Figure 4.3: Three step procedure for uncertainty localization using different types of macroelements.

ure 4.3 by throwing out macroelement 2 in step 1 . The remaining macroelements are then divided to create element based macroelements. This is shown in Figure 4.3 by dividing the remaining group of elements based macroelement 1 into two element based macroelements. A similar procedure is used in step 2 of localizing the macroelements, and throwing out those macroelements which do not contain large modeling errors. And finally step 3 is performed to localize errors within each of the finite elements, such as an error in modeling of the modulus of elasticity, density, or gravity effects.

In order to achieve the objective of finding the most accurate and physical mass and stiffness errors, given errors in the eigenvalues and eigenvectors, the localization must be performed at the sub-element level. The three step procedure proposed above, however, acknowledges the practical case of a small number of measurements. The discussion of macroelements at the sub-element, element, and groups of elements levels is given in the previous section. A localization is performed at each step and must use an overdetermined set of equations in the pseudoinverse. Note that depending on the number of measured errors, the practical strategy could start with element or sub-element based macroelements, rather than groups of elements.

The reduction in macroelements can be accomplished by examining the weighted pseudoinverse, which is found by solving the weighted least squares problem. Defining 
an error using Equation 4.36

$$
e_{\mathrm{N}}=\mathrm{E}\left[\begin{array}{c}
\operatorname{vec}\left\{\Delta \lambda_{\mathrm{N} i}\right\} \\
\operatorname{vec} \Delta \phi_{\mathrm{N} i}
\end{array}\right]-S_{\mathrm{N}} \mathrm{E}\left[\begin{array}{c}
\alpha_{\mathrm{N}} \\
\beta_{\mathrm{N}}
\end{array}\right]
$$

A weighted quadratic cost can then be defined as

$$
J=e_{\mathrm{N}}^{T} W_{\mathrm{N}}^{2} e_{\mathrm{N}}
$$

where $W_{N}$ is the weighting matrix defined in Equation 4.38. The solution to this weighted quadratic minimization is the weighted pseudoinverse.

A procedure is then developed for the reduction in the number of macroelements. First, the pseudoinverse is calculated, and the cost of the weighted least squares problem in Equation 4.49 is evaluated. Then, one macroelement is eliminated at a time, and the cost is evaluated again. The macroelement which reduces the cost the least is then eliminated. This procedure is repeated until there is only one macroelement left. Figure 4.4 shows a sample plot of the normalized cost at each iteration of this procedure.

The elimination procedure also gives a rough ordering of the importance of the macroelements to the pseudoinverse, and therefore their importance to the uncertainty localization. The usual cut off point for reducing the number of macroelements is the point which the cost begins to increase. This is shown to be at macroelement 6 in Figure 4.4. This may have to be changed, however, if there are a lot of uncertainties.

Note that a distinct, but similar procedure can be developed for the reduction of macroelements when localizing variance uncertainties. Using Equation 4.36, a matrix error can be defined

$$
e_{\mathrm{N}}=\operatorname{Cov}\left[\begin{array}{c}
\operatorname{vec}\left\{\Delta \lambda_{\mathrm{N} i}\right\} \\
\operatorname{vec} \Delta \phi_{\mathrm{N} i}
\end{array}\right]-S_{\mathrm{N}} \operatorname{Cov}\left[\begin{array}{c}
\alpha_{\mathrm{N}} \\
\beta_{\mathrm{N}}
\end{array}\right] S_{\mathrm{N}}^{T}
$$

A weighted scalar quadratic cost that is the two norm of the matrix error can then be defined for the reduction of macroelements.

$$
J=\left\|W_{\mathrm{N}} e_{\mathrm{N}}\right\|_{2}
$$




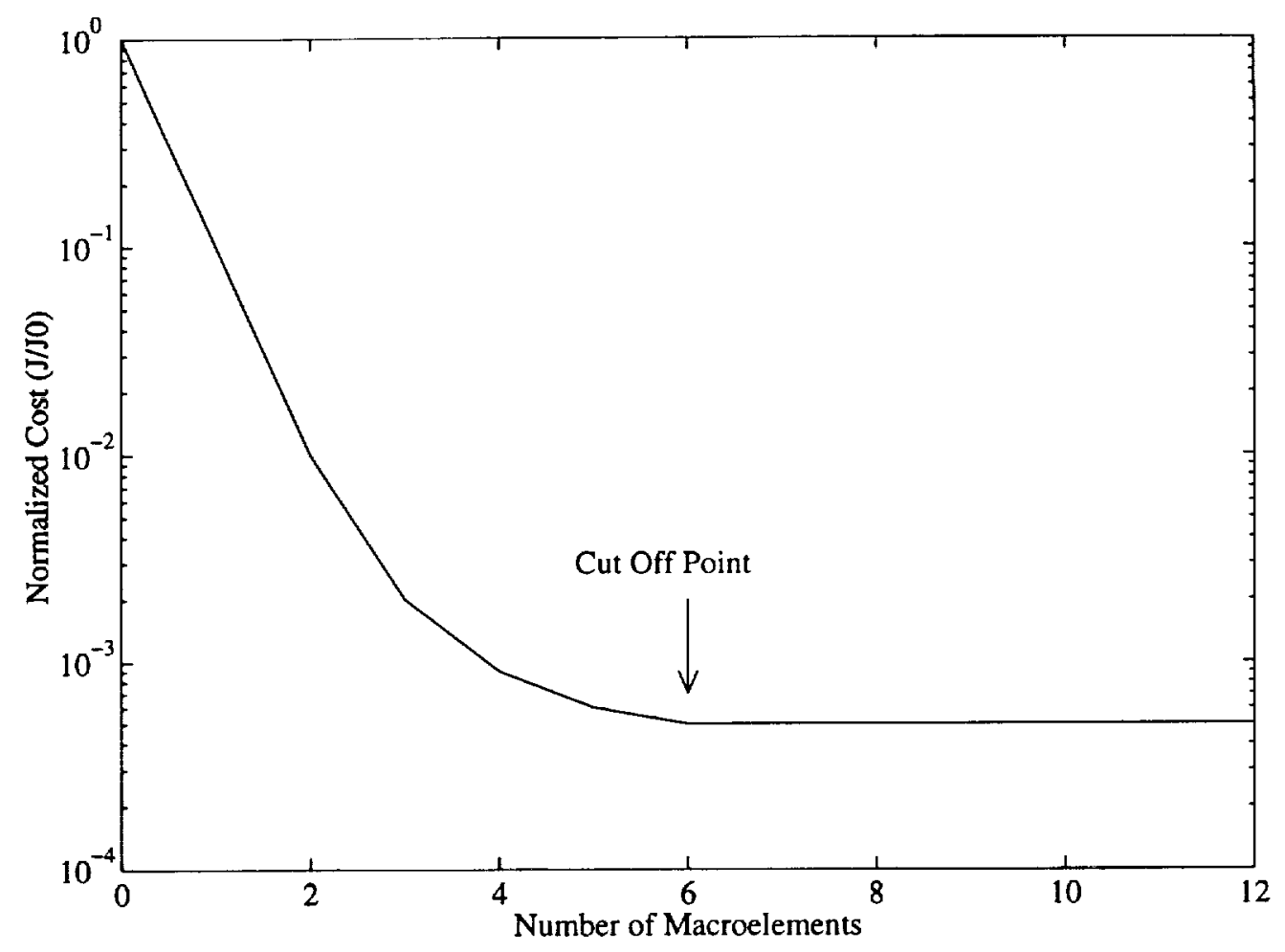

Figure 4.4: Example of the reduction of macroelements using the weighted least squares cost

In this work, the pseudoinverse used is based on the singular value decomposition. Given the weighted sensitivity matrix, a singular value decomposition is performed.

$$
W_{\mathrm{N}} S_{\mathrm{N}}=U_{\mathrm{N}} \Sigma_{\mathrm{N}} V_{\mathrm{N}}^{H}
$$

where $U_{\mathrm{N}}$ and $V_{\mathrm{N}}$ are left and right unitary matrices, and $\Sigma_{\mathrm{N}}$ is a matrix of singular values. The singular value based pseudoinverse is then

$$
\left[W_{\mathrm{N}} S_{\mathrm{N}}\right]^{+}=\sum_{i=1}^{k_{\mathrm{N}}+m_{\mathrm{N}}} \frac{1}{\sigma_{i}} v_{i} u_{i}^{H} \text { for } \sigma_{i}>\epsilon
$$

The value of $\epsilon$ is chosen to reflect the conditioning of the sensitivity matrix.

The last aspect of the practical uncertainty localization and projection strategy is the effects of using a first order linear perturbation to the structural eigenvalue problem, which is inherently nonlinear. If the perturbations are small, the strategy works quite well. However, when they become large, the assumptions made during the derivation become invalid. One way to eliminate these problems is to set up an 
iterative process. In this process, the mean errors are localized using the original finite element model of the nominal system. Then, a new system is formed with the original finite element model and the localized mass and stiffness errors. An eigenvalue problem is then performed on this new system. The new system is then taken as the finite element model, and another iterations of mean error localization is performed. Note that this is similar to a finite element model update strategy.

If the mass and stiffness errors of the nominal system are large enough to require an iterative procedure using an eigensolution at each step, the same must be done for the uncertainty projection. Therefore, Equation 4.44 is not used to find the projected

mean errors. An eigensolution is developed using the modified finite element model, and the mapped mass and stiffness errors.

Although this technique works quite well with the mean errors, the variance uncertainties or bounds are more difficult. Instead of an eigenvalue problem, a solution must be found for a nonlinear statistics problem, which is quite difficult. The variance uncertainties are therefore found using the linear solutions developed previously (Equations 4.40 and Equation 4.45).

Figure 4.5 shows a summary of the practical uncertainty localization and projection strategy. Note that the convergence of the iterative procedure can be checked by examining the mean errors and the converging finite element model.

\subsection{Small Order Example}

An example was sought for this thesis which had the following properties: simple, easy to understand, easy to change parameters, yet complicated enough such that the bending and axial modes were important, and there is a system change that is analogous to the 1-g to $0-\mathrm{g}$ change in environment which is one of the central ideas of this work.. Figure 4.6 shows the small order example that is used to analyze the techniques developed and to add insight to the uncertainty localization and projection problems.

The small order example is a planar system with 4 struts and three collars. Each 


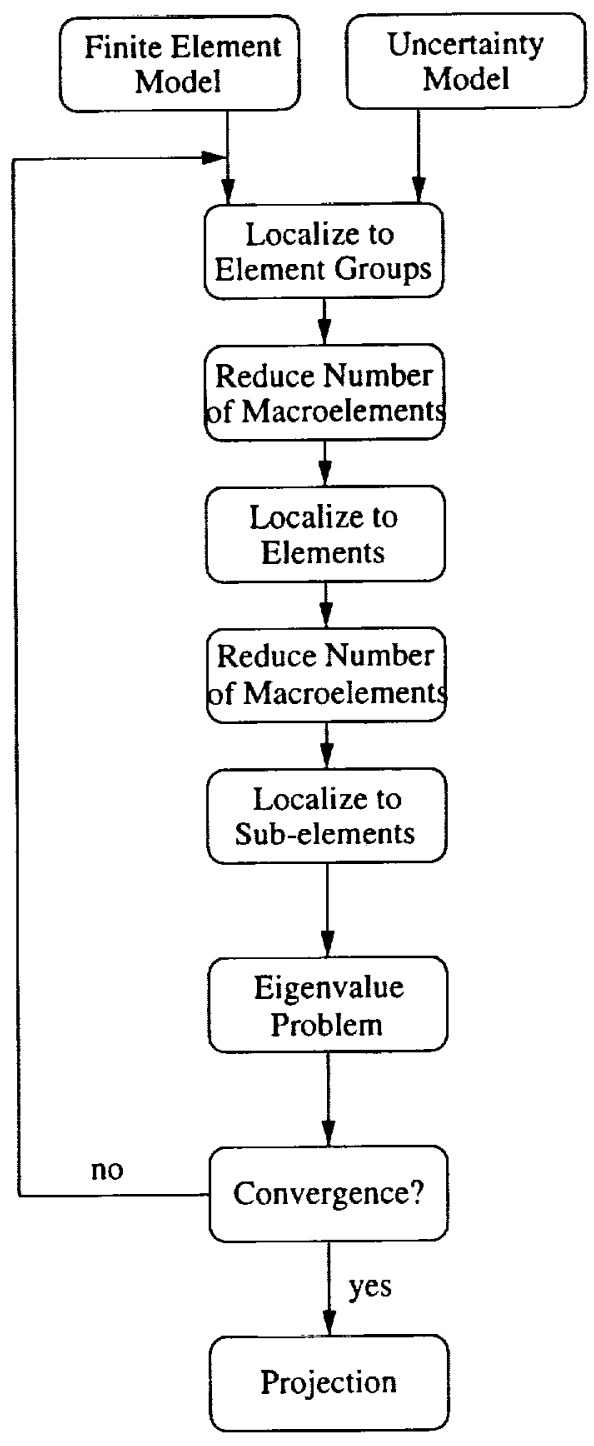

Figure 4.5: Flowchart for the practical implementation of the uncertainty localization and projection methods.

strut is a circular tube, and is divided into 2 elements, for a total of 8 beam elements. There are 8 nodal points with 3 degrees of freedom for each (vertical, horizontal, and rotational) giving 24 degrees of freedom. Table 4.1 summarizes the beam element properties. The mass and stiffness matrices for these elements are standard displacement based two-dimensional beam elements [65]. The mass matrix is chosen to be in consistent form.

The small order example is an "L" shape in order to couple the vertical and horizontal displacements of the elements. A straight cantilever beam decouples the 


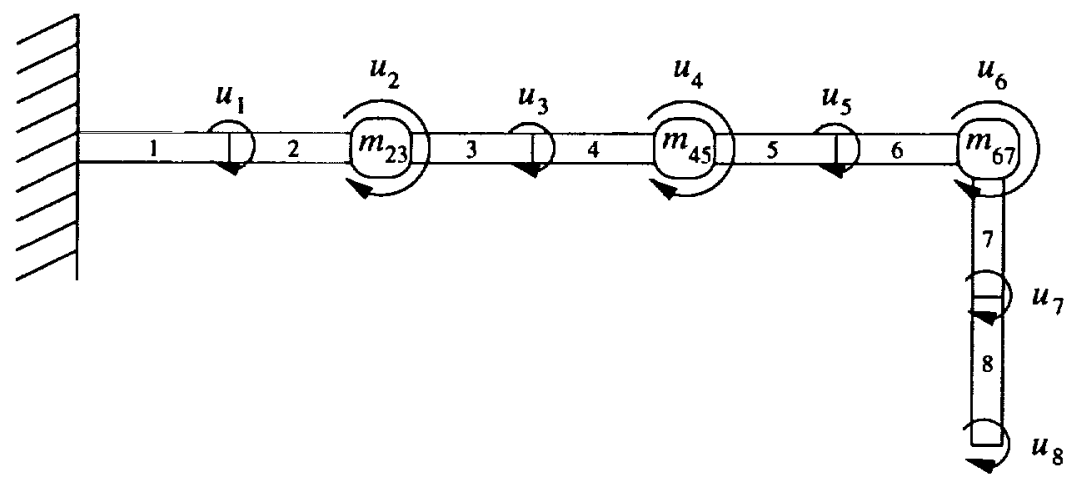

Figure 4.6: Small order example: cantilevered "L" beam

vertical and horizontal displacements. There are also three nodes that contain concentrated masses and inertias. A description of the nodal properties is also given in Table 4.1.

This small order example is also used to verify the projection techniques proposed in this thesis. The change that is analogous to that of the $1-\mathrm{g}$ to $0-\mathrm{g}$ environment change is a cantilevered configuration to free-free configuration. The free-free configuration has 9 nodal points giving 27 degrees of freedom. Table 4.2 gives a summary of the first seven frequencies of each system. The damping is assumed to be proportional and $1 \%$ for all modes in both the cantilevered and free-free systems.

In order to validate the methods presented, and to gain insights into the practical situations in which the methods might perform well or poorly, three distinct cases

\begin{tabular}{l|c} 
Property & Value \\
\hline \hline Modulus of Elasticity $(E)$ & $2.7 \times 10^{9} \mathrm{~N} / \mathrm{m}^{2}$ \\
Density $(\rho)$ & $2021 \mathrm{~kg} / \mathrm{m}^{3}$ \\
Outer Radius $(r)$ & $0.0128 \mathrm{~m}$ \\
Thickness $(t)$ & $0.0032 \mathrm{~m}$ \\
Length $(L)$ & $0.125 \mathrm{~m}$ \\
Nodal Mass $(m)$ & $0.6 \mathrm{~kg}$ \\
Nodal Inertia $(I)$ & $0.004 \mathrm{~m}^{4}$
\end{tabular}

Table 4.1: Beam sectional and nodal properties for the small order example 
are presented for the small order example. The first is when complete measurement of all degrees of freedom and frequencies are available. This verifies that the methods presented work for the best case. The second case is a more realistic scenario where there are 4 frequencies and 4 degrees of freedom measured that are evenly spaced within the system. These are the rotational degrees of freedom $u_{1}, u_{3}, u_{5}$, and $u_{7}$ in Figure 4.6. The final case is when there are 4 frequencies and 4 degrees of freedom measured, but the degrees of freedom are concentrated in one area, while errors exist in another area. The degrees of freedom are on the left side of the example, i.e. $u_{1}, u_{2}, u_{3}$, and $u_{4}$ in Figure 4.6, and the uncertainties are scattered throughout, included the right side. A summary of these test cases is given in Table 4.3.

In addition to the topology of the test cases, Table 4.3 also shows the uncertainties (both deterministic and stochastic) introduced into the system. The errors are presented in the form of a normal variable with mean and standard deviation errors in percentage, or $(\cdot)=N\left(\overline{\Delta(\cdot)}, \sigma_{\Delta(\cdot)}\right)$. The sample problem is examined by sampling the stochastic uncertainties, introducing all uncertainties into the model, and simulating a large number of cases in a Monte Carlo type simulation. Case 1 examines mean and variance uncertainties separately, while Cases 2 and 3 examine a variety of uncertainties throughout the structure.

\begin{tabular}{|c|c|}
\hline \hline Cantilever $(\mathrm{Hz})$ & Free-Free $(\mathrm{Hz})$ \\
\hline 3.1 & 0.0 \\
\hline 18.2 & 0.0 \\
\hline 43.1 & 0.0 \\
\hline 72.8 & 22.3 \\
\hline 97.3 & 44.8 \\
\hline 120.2 & 83.3 \\
\hline 131.2 & 110.3 \\
\hline
\end{tabular}

Table 4.2: Frequencies for the cantilevered and free-free configurations of the small order example 


\begin{tabular}{c|c|c} 
& Uncertainties & Measurements \\
\hline \hline Case 1A & $E_{2}=N(5 \%, 0)$ & All frequencies \\
& $\rho_{2}=N(-5 \%, 0)$ & All DOF \\
\hline Case 1B & $E_{2}=N(0,2 \%)$ & All frequencies \\
& $\rho_{2}=N(0,-2 \%)$ & All DOF \\
\hline Case 2 & $E_{2}=N(5 \%, 2 \%)$ & 4 frequencies \\
& $L_{4}=N(-5 \%, 0)$ & $u_{1}, u_{3}, u_{5}, u_{7}$ \\
& $r_{6}=N(-5 \%, 2 \%)$ & \\
& $m_{67}=N(3 \%, 0)$ & \\
\hline Case 3 & $E_{2}=N(5 \%, 2 \%)$ & 4 frequencies \\
& $L_{4}=N(-5 \%, 0)$ & $u_{1}, u_{2}, u_{3}, u_{4}$ \\
& $r_{6}=N(-5 \%, 2 \%)$ & \\
& $m_{67}=N(3 \%, 0)$ &
\end{tabular}

Table 4.3: Test cases for the small order example.

\subsubsection{Case 1: Complete Measurements}

The first test case examined is the ability of both methods to localize both mean error and variance errors in the nominal system, and project them into the modified system using complete measurements, i.e. measuring all of the frequencies and mode shapes. This gives $24+24 \times 24=600$ pieces of information. Case $1 \mathrm{~A}$, as shown in Table 4.3, examines the localization and projection of mean error uncertainties only. The uncertainties in this case are a $5 \%$ mean error in the modulus of elasticity of element 2 , and a $-5 \%$ mean error in the density of element 2 . This case is particularly interesting both mass and stiffness mean errors are introduced into the same finite element, adding more complexity to the localization process.

Figure 4.7 shows a summary of the mean error localization and projection for Case $1 \mathrm{~A}$ using the matrix method. Figure 4.7(a) shows three dimensional plots of the actual mass and stiffness errors and localized mean errors found using the matrix method, as a function of the degrees of freedom. Notice the agreement is practi- 
cally identical. Figure 4.7(b), shows the modified or free-free finite element model freuencies, simulated frequencies (with the uncertainties introduced), and predicted frequencies using the model and projected mean error. Note that all points on the plot are normalized by the corresponding free-free finite element model frequency. The prediction of the mean errors in frequency using the matrix method works very well for this case.

The sensitivity method performed for this case utilizes a two step procedure: localize to elements and reduce the number of elements; localize to sub-elements. Although the number of measured errors is large enough such that this two step procedure is not necessary, it is detailed in this manner to demonstrate the general sensitivity technique. It is also the most commonly used strategy. Note that a one step strategy would not have improved the results.

The first step of the two-step sensitivity method involves localizing to particular finite elements. For this step, each mass (8) and stiffness (8) finite element and each concentrated mass/inertia (3) are used, comprising a total of 19 macroelements. Figure 4.8(a) shows a plot of the cost for the pseudoinverse in the sensitivity method, as each macroelement is eliminated. Notice that if 8 macroelements are used, the pseudoinverse, and therefore the localization method, performs essentially as well as if 19 macroelements are used; and there is only a slight difference in cost when using 4 macroelements. Also notice that the elimination procedure orders the macroelements. This is seen in Figure 4.8(a) as weighted least squares cost is substantially reduced using the first two macroelements. Because there are only two mean errors, the rough ordering of macroelements places the two macroelements that are the modeling error sources first.

The decision is made to use the first 8 macroelements. These 8 macroelements are either mass, stiffness, or nodal mass elements. The retained mass and stiffness macroelements are split into three sub-elements for use in the next step of the localization procedure. Table 2.4 shows the many variations of sub-elements that can be used. For this step of the localization, only three are relavent: $\Delta E, \Delta \rho$, and $\Delta L$. The perturbation $\Delta t$ can be represented with the macroelements chosen, and 

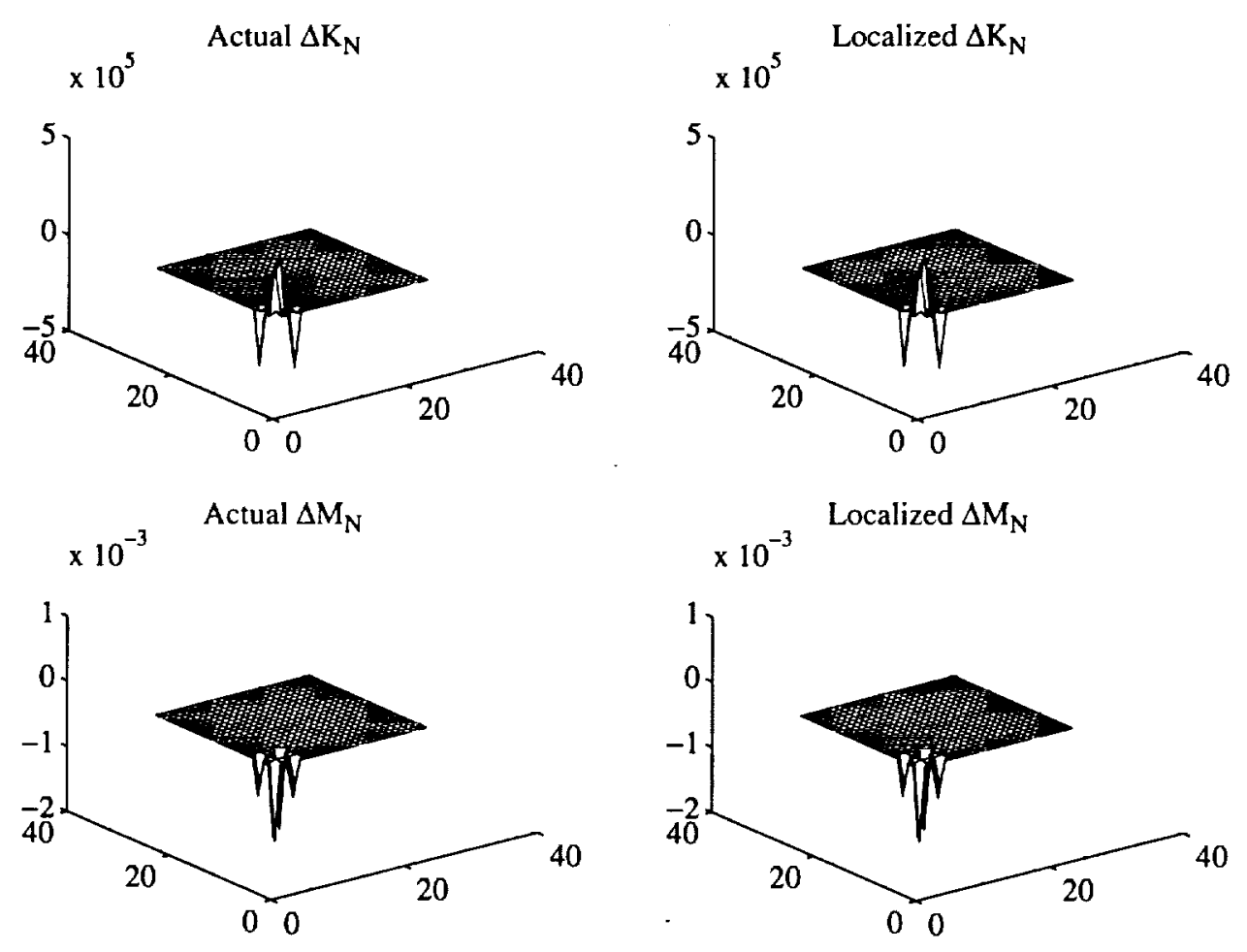

(a) Mass and stiffness mean error matrices.

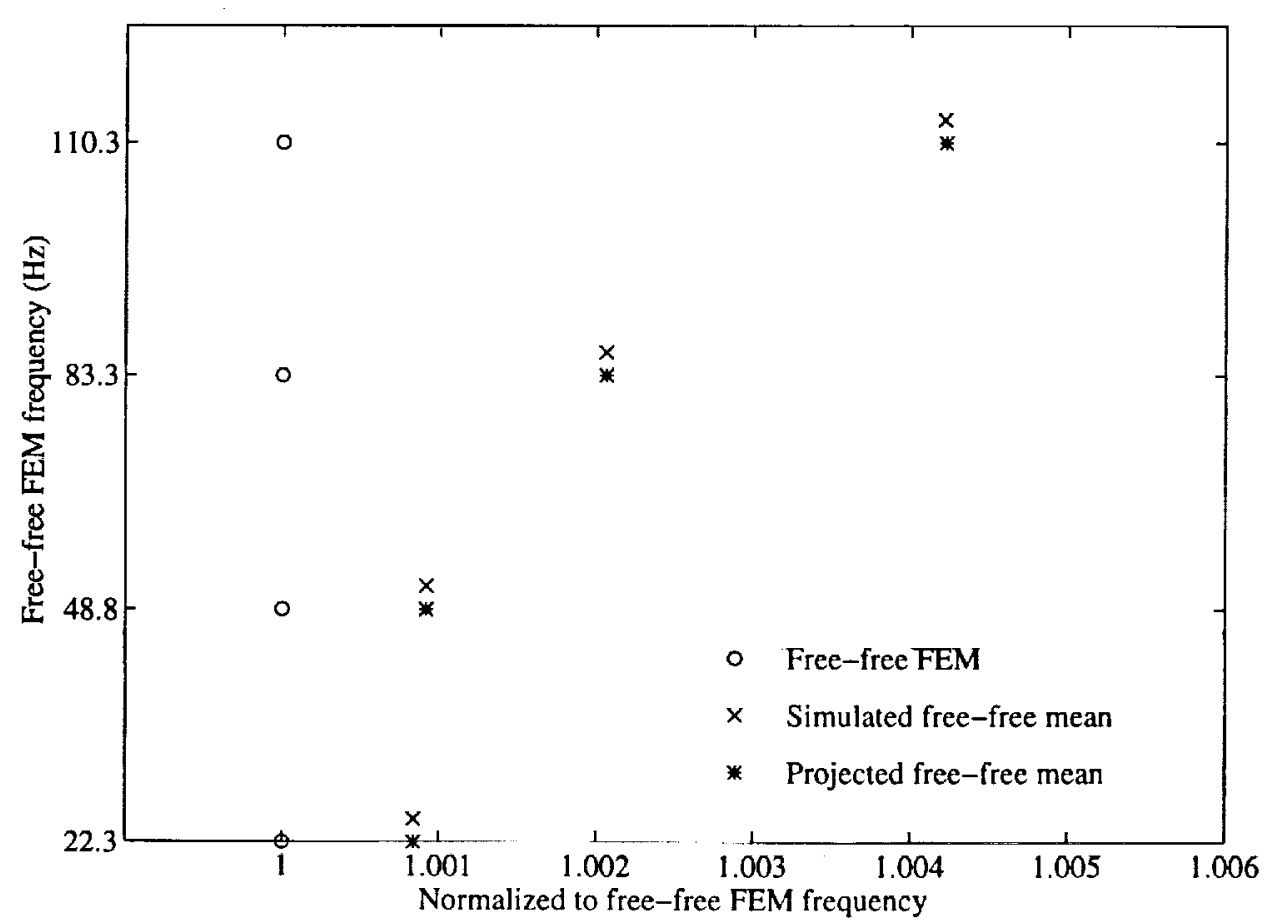

(b) Frequency results for cantilever to free-free projection of mean errors.

Figure 4.7: Matrix method for Case 1A using complete measurements. 
the perturbation $\Delta \nu$ is not applicable for this two-dimensional problem. In addition, the $\Delta r$ perturbation is quite similar to a combination of $\Delta E$ and $\Delta \rho$, except for the extensional degree of freedom. Because the measured degrees of freedom and modes are rotational, the $\Delta r$ perturbation is almost indistiguishable from the $\Delta E$ and $\Delta \rho$ errors.

By examining retained mass and stiffness (and concentrated mass/inertia) element based macroelements from step one, and the possible sub-elements described above, the macroelements of step two of the sensitivity method are then created. A total of 22 macroelements are then used for this step of the process. Figure 4.8(b) shows the weighted least squares cost for this step of the localization strategy as a function of the given macroelements. Notice the first two elements again substantially reduce the cost as a result of ordering the correct two macroelements first.

Figure 4.9 shows a summary of the uncertainty localization and projection for Case $1 \mathrm{~A}$ using the two step sensitivity method. Figure 4.9(a) shows the actual mass and stiffness errors to be predicted correctly, as they were for the matrix method. Figure 4.9(b) shows the frequency results for the modified, or free-free system, which again shows good agreement. The sensitivity method described here uses only one iteration of the general strategy shown in Figure 4.5. If the mean error localization had not performed adequately, additional iterations could have been performed to improve the results. An example of when and how the iteration procedure of the sensitivity method is used is given in a subsequent example.

Case 1B is similar to the previous case, however, variance uncertainties are localized and projected instead of mean errors. Examining variance uncertainties is more difficult than the case with mean errors because localized mass and stiffness uncertainties cannot be described pictorially. For the mean error case, once the macroelemental scale factors are found, and mass and stiffness error matrices can be calculated and compared to the actual error matrices. For the variance uncertainty case, however, this cannot be done. One can only examine the macroclemental scale factors themselves, which are in the form of a covariance matrix. The easiest check to ensure the validity of the localization and projection of variance uncertainties is 


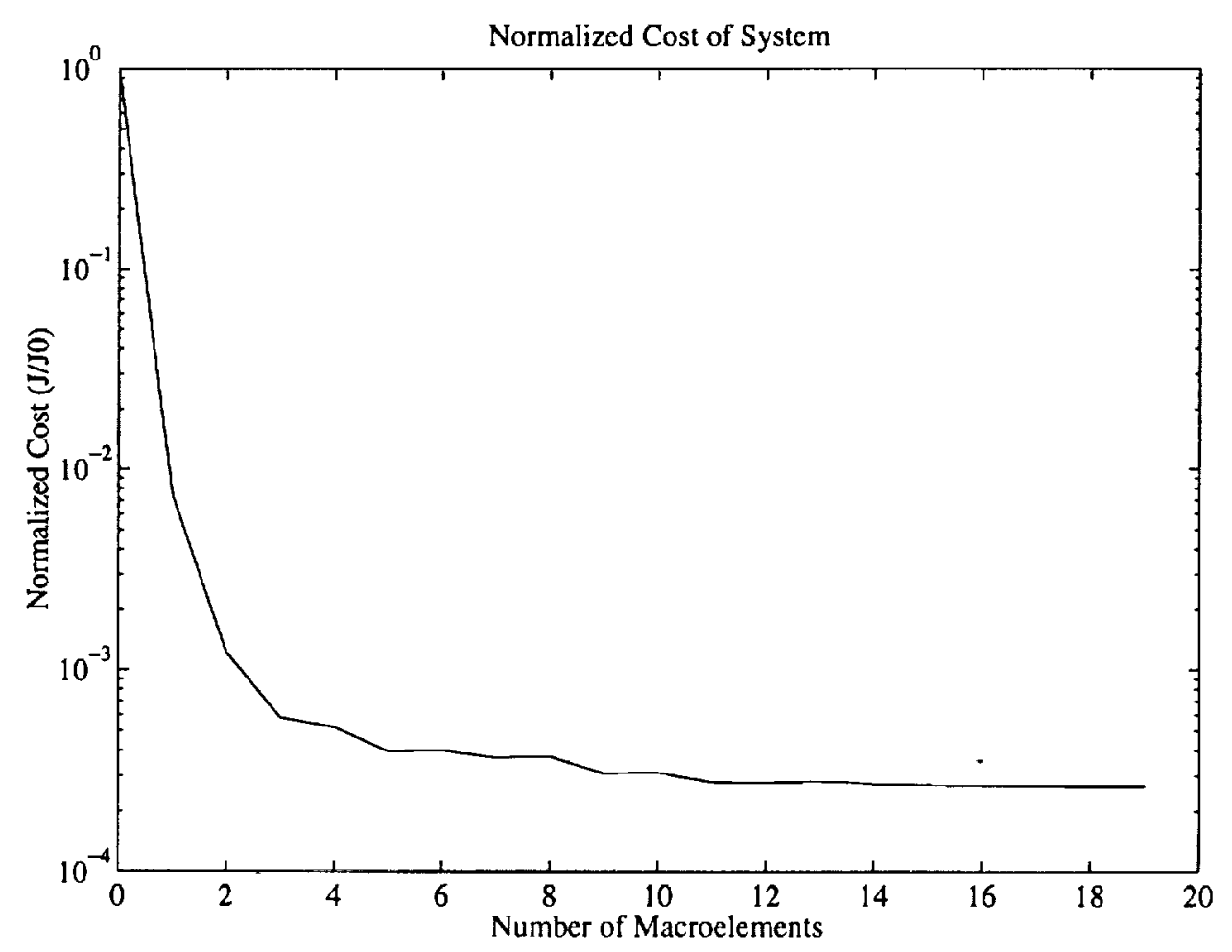

(a) Mean error localization using element based macroelements.

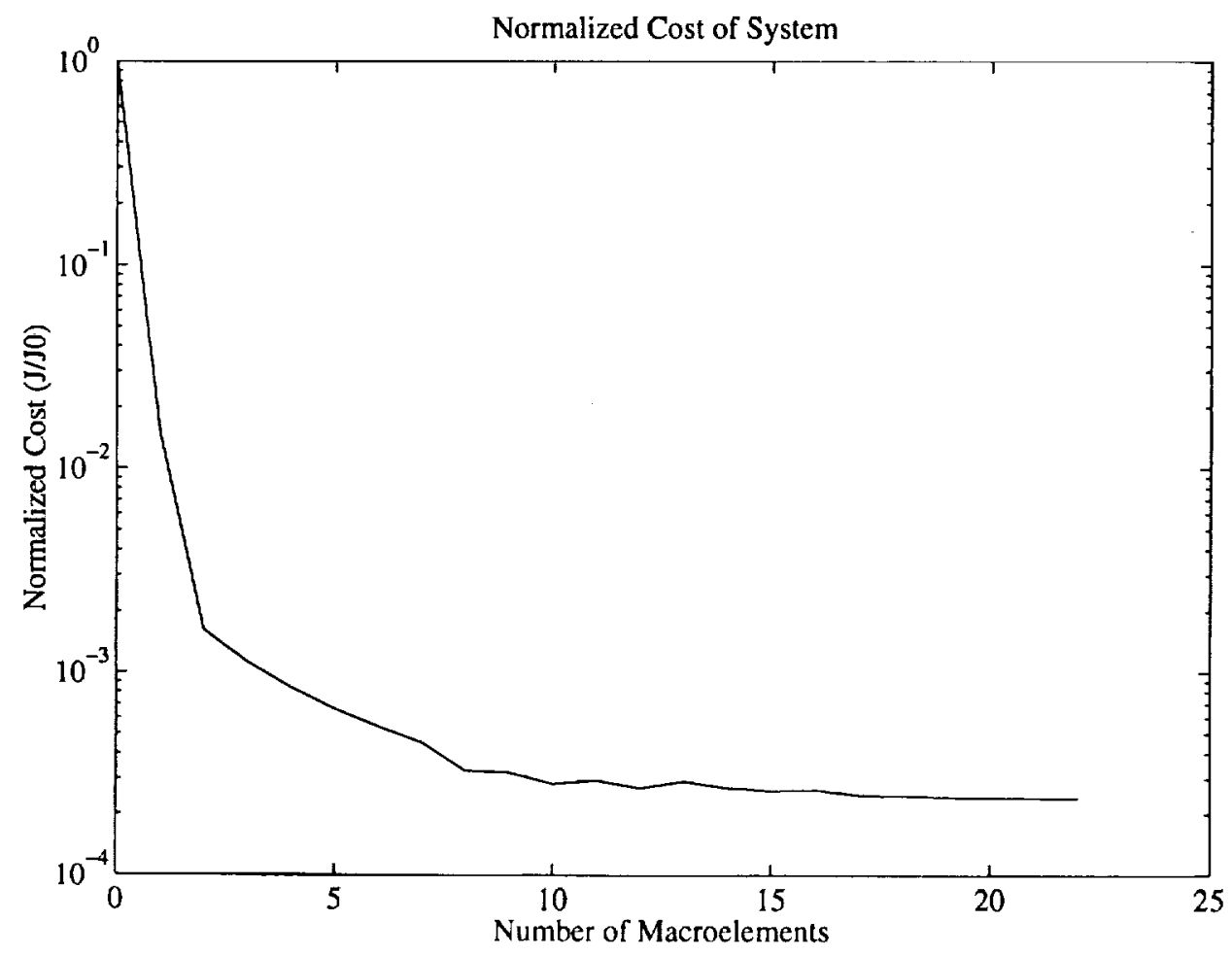

(b) Mean error localization using sub-element based macroclements.

Figure 4.8: Weighted least squares cost showing the possible elimination of macroelements. 

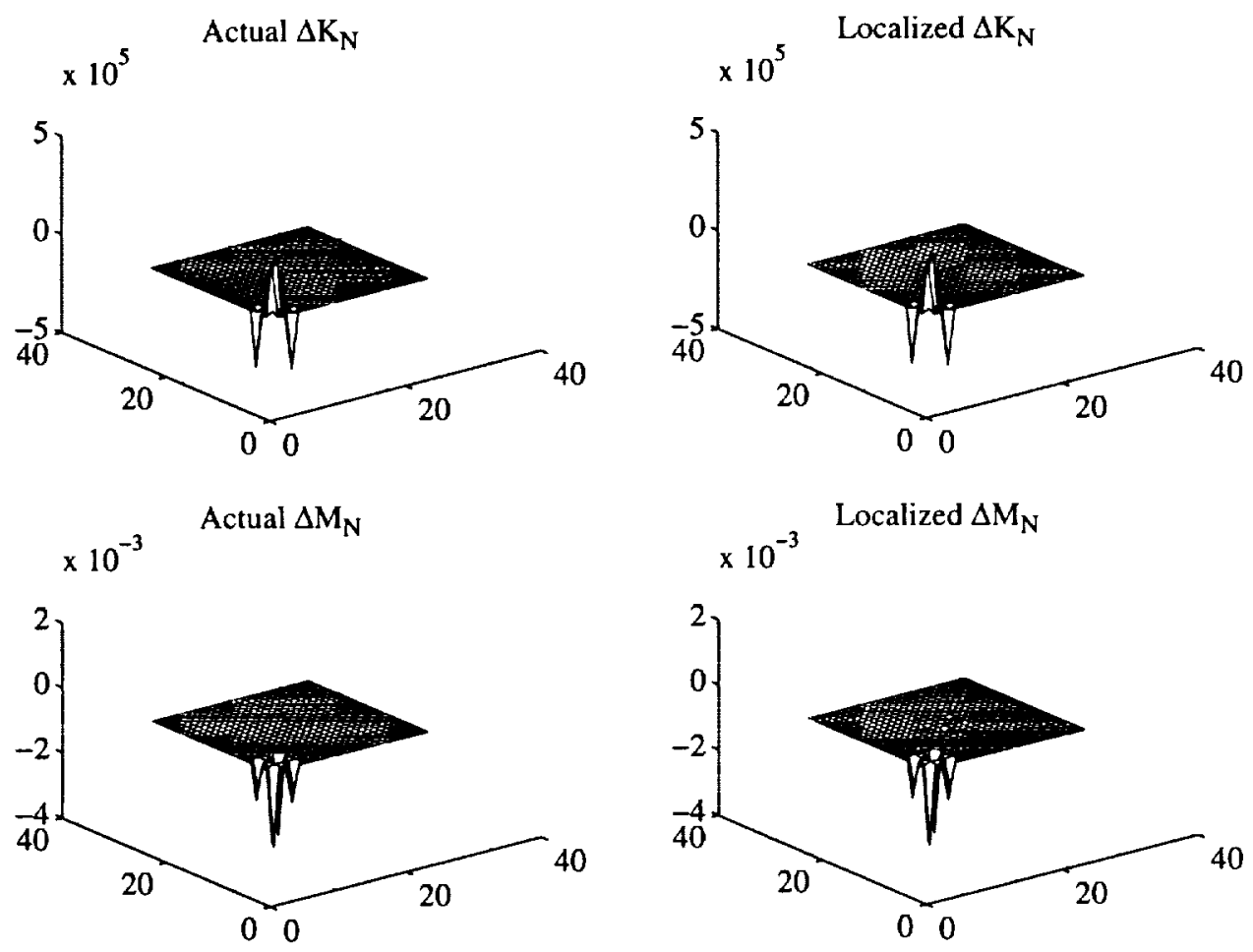

(a) Mass and stiffness mean error matrices.

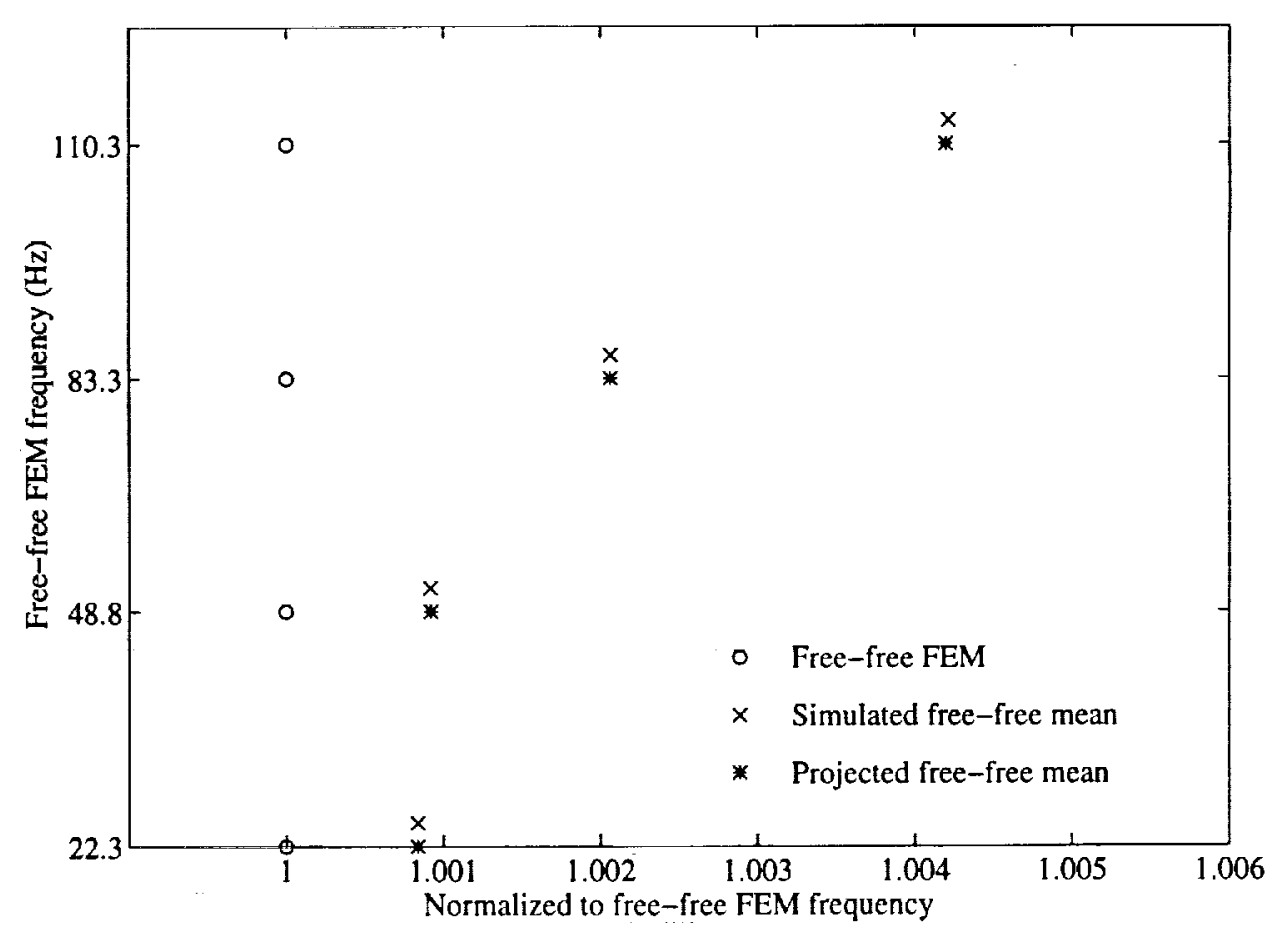

(b) Frequency results for cantilever to free-free projection of mean crrors.

Figure 4.9: Sensitivity method for Case 1A using complete measurements. 
to run many simulated cases while sampling the uncertainties, for both the nominal and modified systems. Then the variance uncertainties of the nominal system can be calculated and projected into the modified environment where they can be compared to the simulated results of the modified system. This is the format for Case $1 \mathrm{~B}$.

As shown in Table 4.3 , Case $1 \mathrm{~B}$ uses a $2 \%$ standard deviation in both the modulus of elasticity and density of element 2 , and there is no mean error. Therefore, in most cases the random error in the modulus of elasticity and density of element 2 is less than $6 \%$. There were 100 cases simulated (with the two variables being random), and the frequencies and modes shapes are calculated for each. Figure 4.10 shows the frequency results of the localization of the variance uncertainties and projection using both the matrix and sensitivity methods for Case 1B. The simulated bounds are calculated from the 100 simulated cases, and the projected bounds are those bounds that are predicted using the uncertainty localization and projection methods. Both methods again work quite well in predicting the bounds for the case when all of the degrees of freedom and frequencies are measured.

The localization of variance uncertainties using the sensitivity method also used a two-step procedure, along with the same macroelements descrbed previously. Note that the matrix error and cost defined in Equations 4.50-4.51.

\subsubsection{Case 2: Partial Measurements}

Case 1 verified that the uncertainty localization and projection methods developed in this thesis work well using measurement of all degrees of freedom and frequencies. One of the primary objectives of this work, however. is to develop methods that also work well for practical problems. Case 2 applies these methods to a more practical case.

Case 2, as shown in Table 4.3, contains only partial measurement of the modal parameters of the system. There are 4 modes measured, and 4 degrees of freedom, giving $4+4 \times 4=20$ total measurements. The 4 degrees of freedom are rotational and are evenly spaced along the beam. These are shown as $u_{1}, u_{3}, u_{5}$, and $u_{7}$ in Figure 4.6. There are uncertainties, both deterministic and stochastic, in four areas 


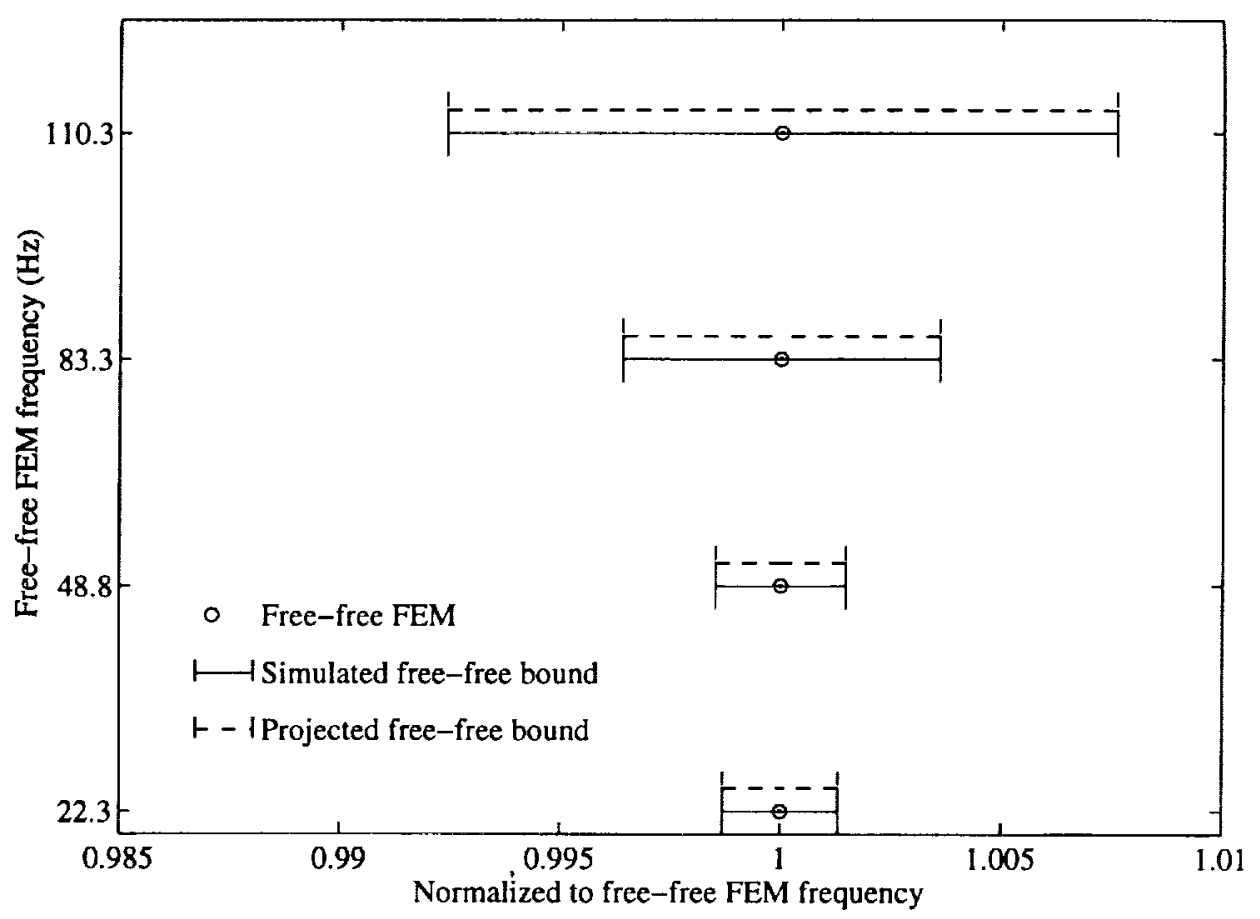

(a) Matrix Method

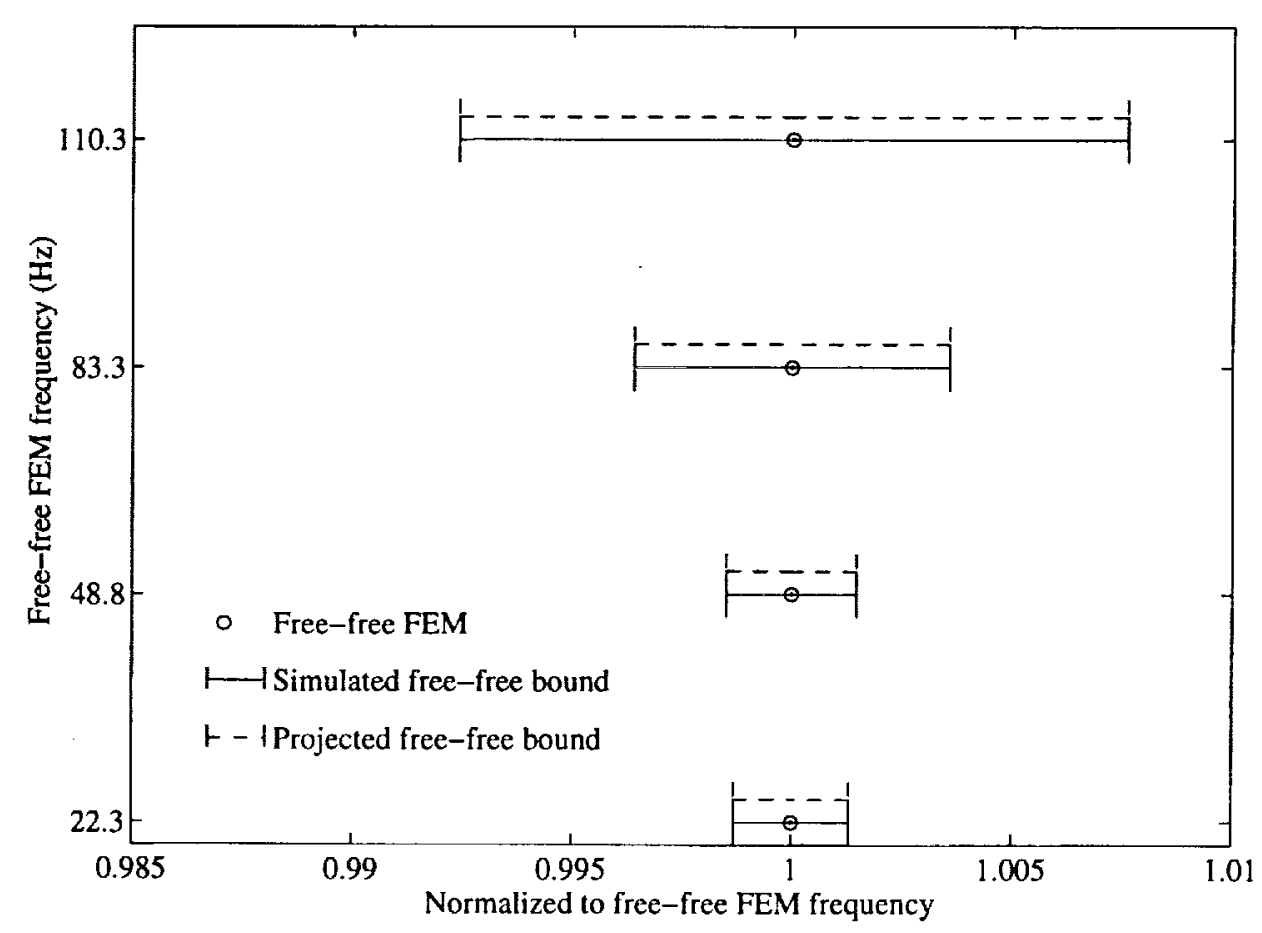

(b) Sensitivity Method

Figure 4.10: Localization and projection of variance uncertainties to predict frequency bounds for Case $1 \mathrm{~B}$ using complete measurements. 
of the structure, as shown in Table 4.3. These four areas are spread throughout the structure: the modulus of element 2, the length of element 4 , the outer radius of element 6 , and the mass and inertia of the node (collar) between elements 6 and 7 . For Case 2, 10 simulated cases are run for both the nominal and modified systems, with the mean error and variance uncertainties in measured frequencies and mode shapes calculated for each. The nominal mean error and variance uncertainties are then localized and projected into the modified system to predict the corresponding uncertainty model. These predictions can then be checked by comparing the 10 simulated cases of the modified system.

Figure 4.11 shows the localized and actual mass and stiffness error matrices using the matrix method. The localized mass and stiffness error matrices do not resemble the actual error matrices. The matrix method supplies mass and stiffness error matrices that are not physical, resulting from the use of a pseudoinverse on an overdetermined system in the derivation. The results still solve the given optimization problem, however. Predictably, when these error matrices are projected into the modified, or free-free system, the predicted results are extremely poor, such that they are not presented here.

Figure 4.12 shows the results of the sensitivity method for Case 2. The two-step localization procedure similar to that used previously is employed, i.e. the same element and sub-element based macroelements. Figure 4.12(a) shows the predicted and actual mass and stiffness mean error matrices. The agreement using the sensitivity method is excellent. Figure 4.12(b) shows the frequency results of projecting of both the mean error and variance uncertainties into the modified system to predict the freefree frequencies and bounds. Shown are the FEM frequencies, projected frequency and bound for four sample frequencies, and 10 simulated cases of for the free-free system. The sensitivity method works quite well in predicting both the mean errors and bounds, such that each of the simulated cases are within the predictions. The sensitivity method works well because both the modeling error sources are correctly spanned by chosen macroelements and the pseudoinverse used is on an overdetermined system. 

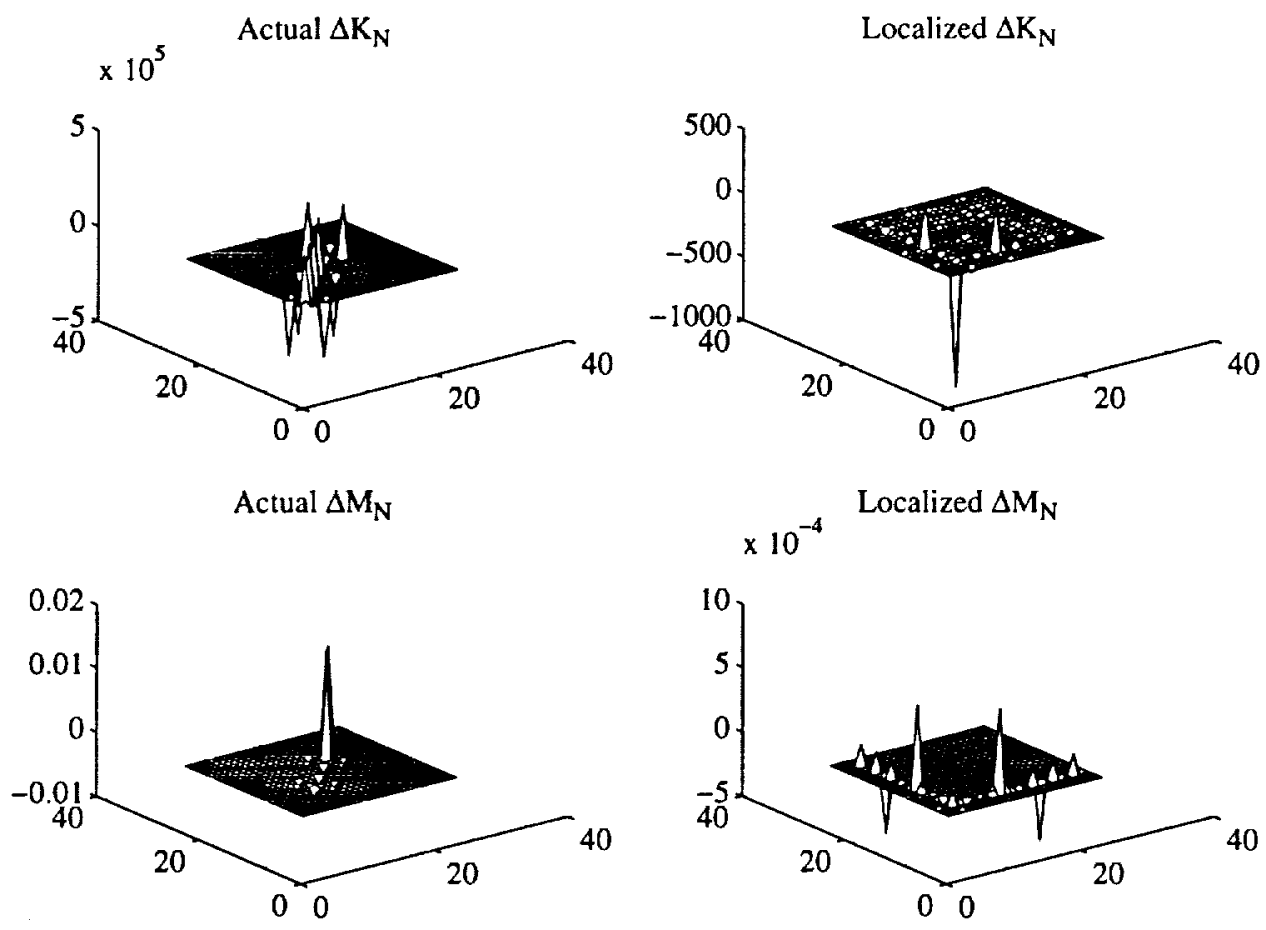

Figure 4.11: Matrix Method for Case 2 using partial measurements.

\subsubsection{Case 3: Partial Measurements}

The final case examined uses the same uncertainties as in the previous case, but the measurements are now on the first four nodes. This is shown in Figure 4.6 as four rotational degrees of freedom $u_{1}, u_{2}, u_{3}$, and $u_{4}$. This implies that a few of the uncertainties, i.e. $r_{6}$ in element 6 and $m_{6.7}$ do not have measured degrees of freedom near the unmodeled area. This case demonstrates the ability of the methods to localize errors in areas of the structure that are not completely observable to the sensor measurements.

For this case, the matrix method produced results that were inconsistent, similar to those for Case 2. They are therefore not presented here. Figure 4.13 shows the mass and stiffness mean error matrices, and projection for the sensitivity method. The localized mass and stiffness mean errors are quite similar to the actual errors, though they are not identical. The predicted mass error in the $m_{6.7}$ is larger, while the predicted stiffness error matrix is smaller compared to the actual mass and stiffness 


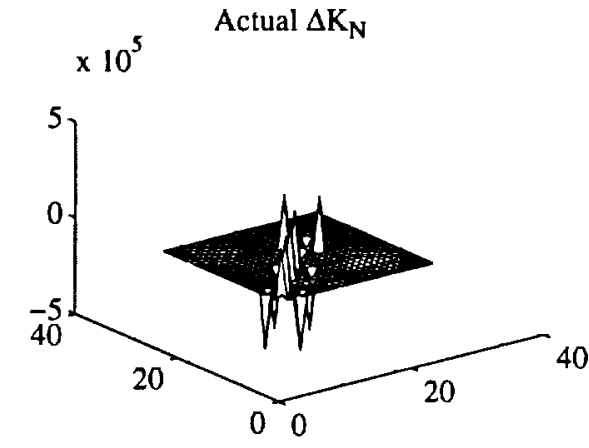

Actual $\Delta \mathrm{M}_{\mathrm{N}}$

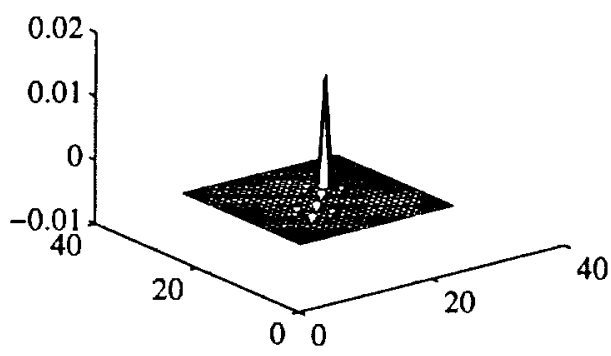

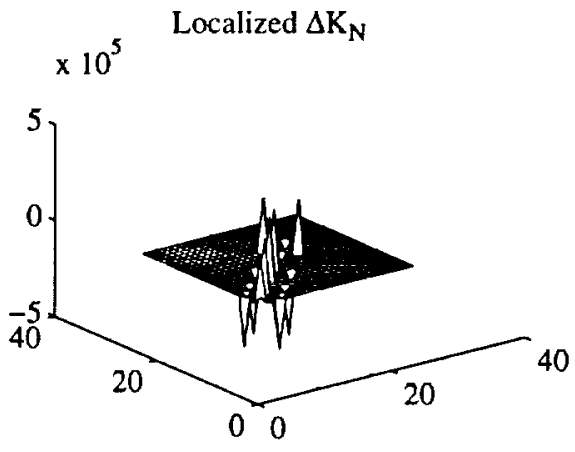

Localized $\Delta \mathrm{M}_{\mathrm{N}}$

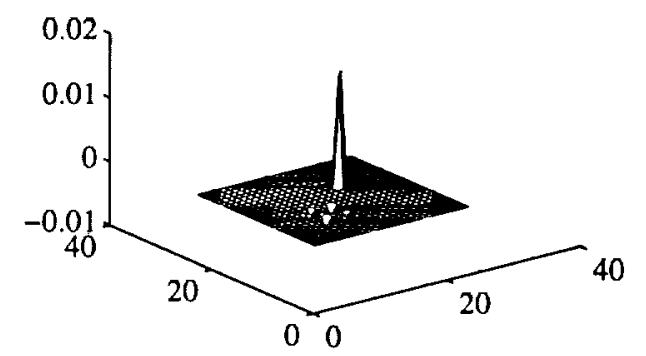

(a) Mass and stiffness mean error matrices.

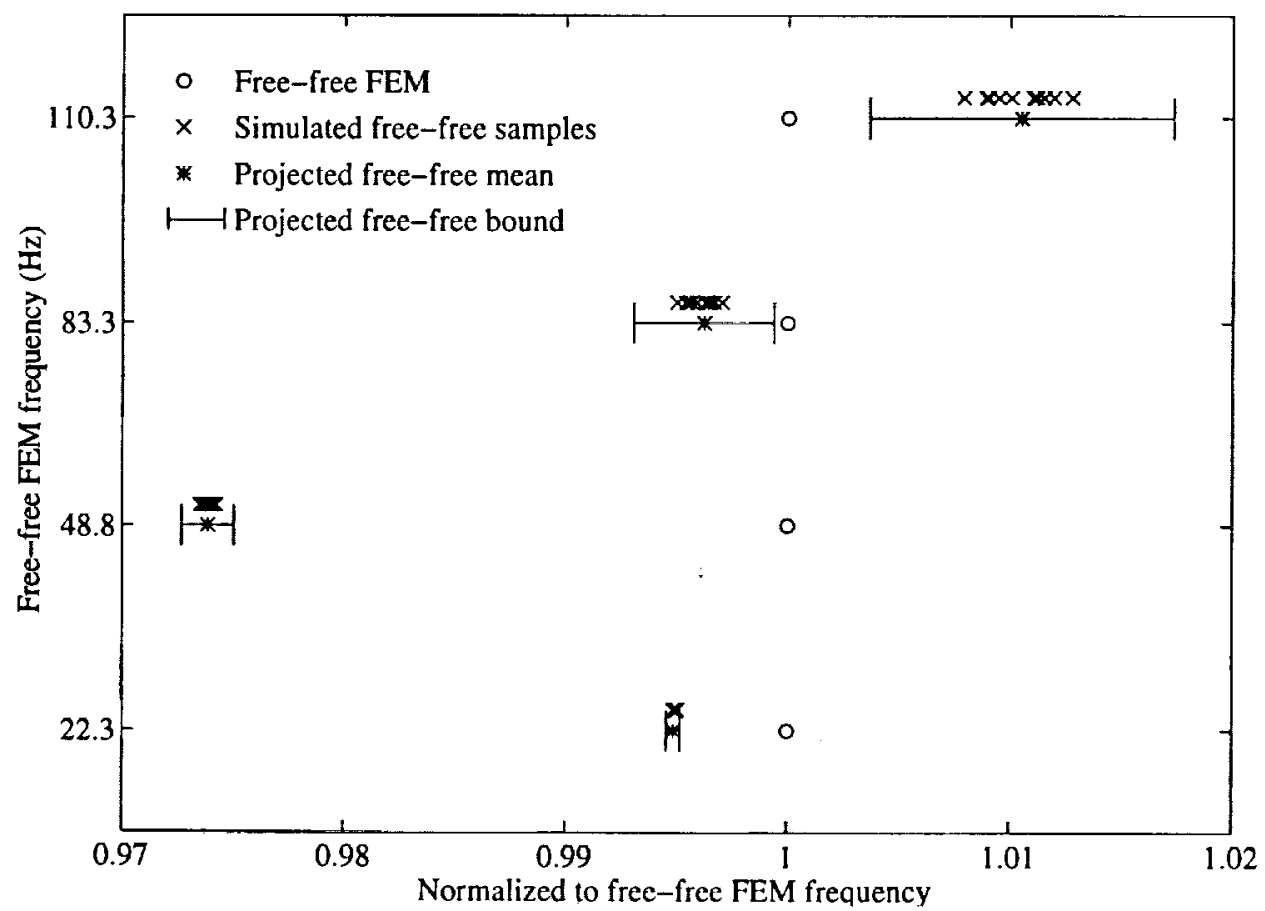

(b) Frequency results for cantilever to free-free projection of mean error and variance uncertainties.

Figure 4.12: Sensitivity Method for Case 2 using partial measurements. 
errors. This can also be seen in the projection, shown in Figure 4.13(b). While the predicted mean errors are quite good, they do not predict the range of all of the simulated frequencies. For the $22.3 \mathrm{~Hz}$ mode, the predicted mean error shifts the model near the simulated frequency samples, but there still remains a residual error such that the predicted mean error and bound does not match the simulated free-free samples for any case.

In order to accurately predict both the mean errors and bounds, the iterative strategy proposed in the development of the sensitivity method is implemented. This general strategy is shown in Figure 4.5. For this case, 5 iterations of the two step uncertainty localization process are implemented. Figure 4.14 plots the updated cantilevered and projected free-free mean frequencies (-), along with the simulated mean $(x)$ and actual frequencies (o), as a function of iteration. Note each point is normalized to the actual frequencies. Therefore, the parameters should converge to the simulated mean, instead of the actual parameter value. In one iteration, the sensitivity method works well, but a few of the frequencies have not converged to their respective simulated means. After two iterations, however, the method predicts the simulated mean frequencies quite well. And after 5 iterations, it works very well.

Figure 4.15 shows the results of this uncertainty localization and projection after using 5 iterations (Compared to the analogous plots after using 1 iteration in Figure 4.13). Figure 4.15(a) shows the mass and stiffness mean error matrices are now localized quite well. In addition, Figure 4.15(b) shows the projected mean errors and bounds for the frequencies of the free-free system now predicted the simulated samples as well. This example demonstrates that the sensitivity method works quite well on the first iteration as a course adjustment, but may, on a few occasions, need a few more iterations for fine adjustments. The best manner in which to predict when multiple iterations are needed is to examine each nominal mean error localization. If the updated system matches the mean of the nominal data quite well, then another iteration is not needed. If, however, there are still small residual errors, a few more iterations will improve both the localized error matrices and projection procedure. 


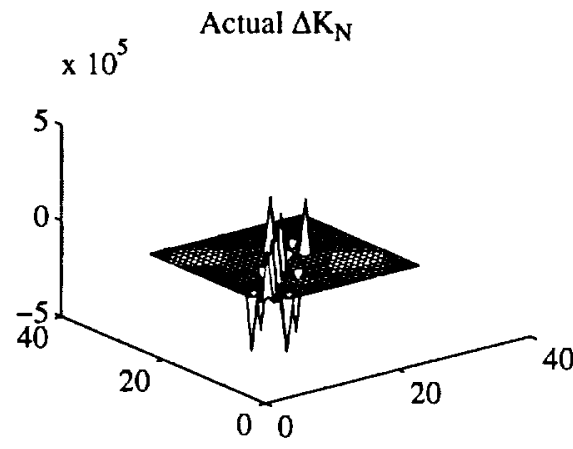

Actual $\Delta \mathrm{M}_{\mathrm{N}}$

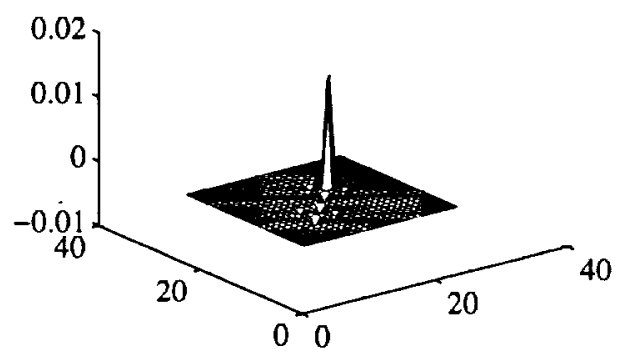

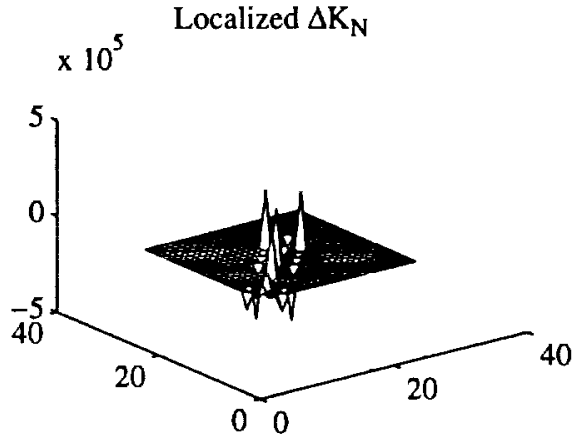

Localized $\Delta \mathrm{M}_{\mathrm{N}}$

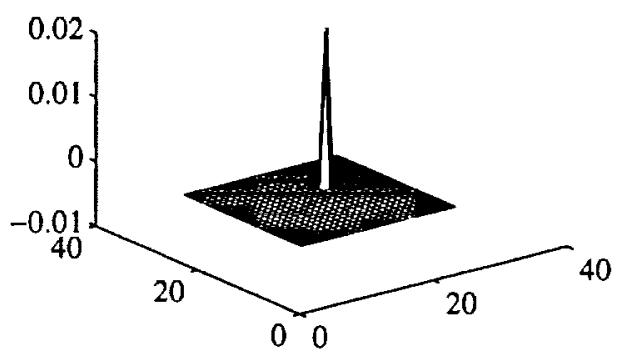

(a) Mass and stiffness mean error matrices.

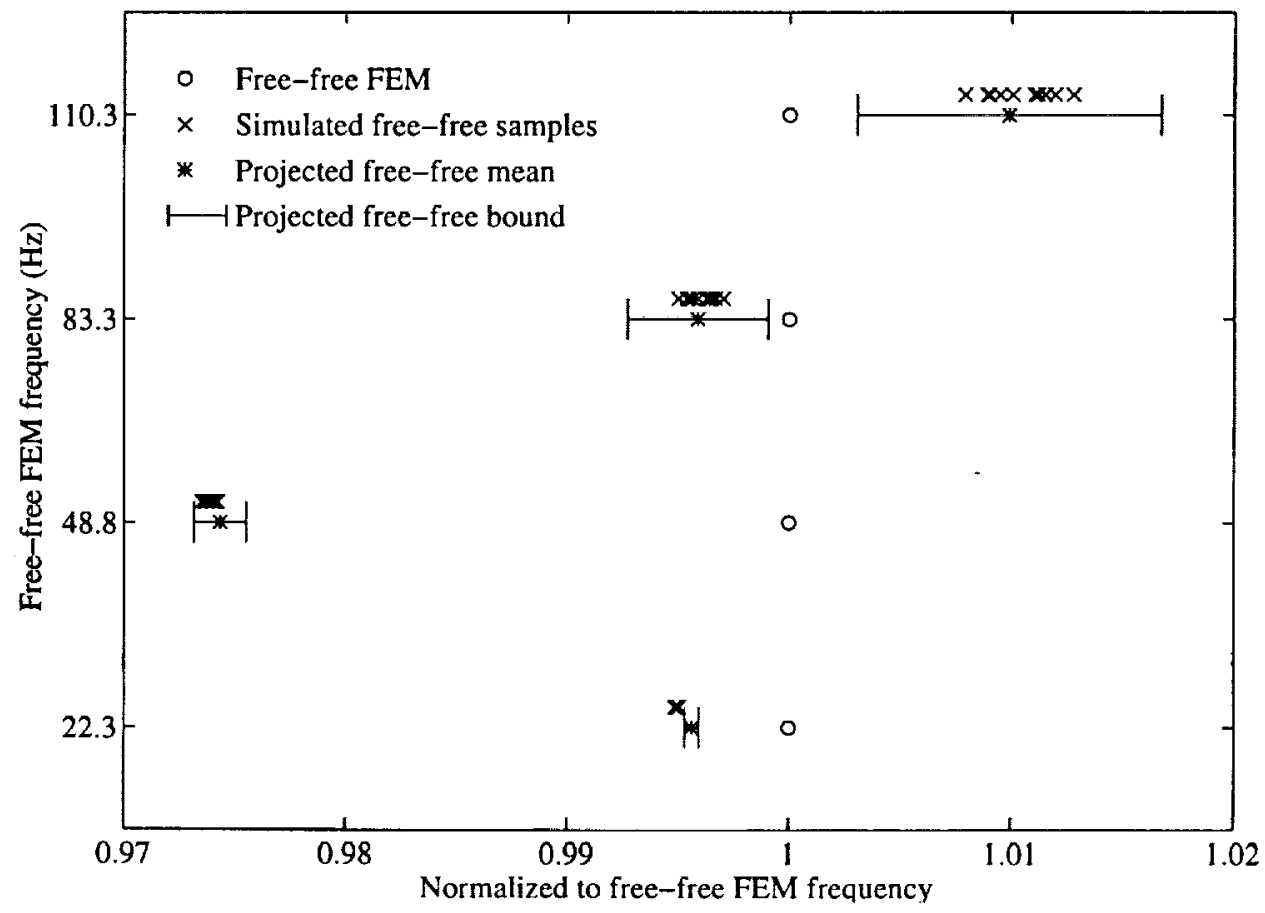

(b) Frequency results for cantilever to free-free projection of mean error and variance uncertainties.

Figure 4.13: Sensitivity Method for Case 3 using partial measurements and one iteration. 


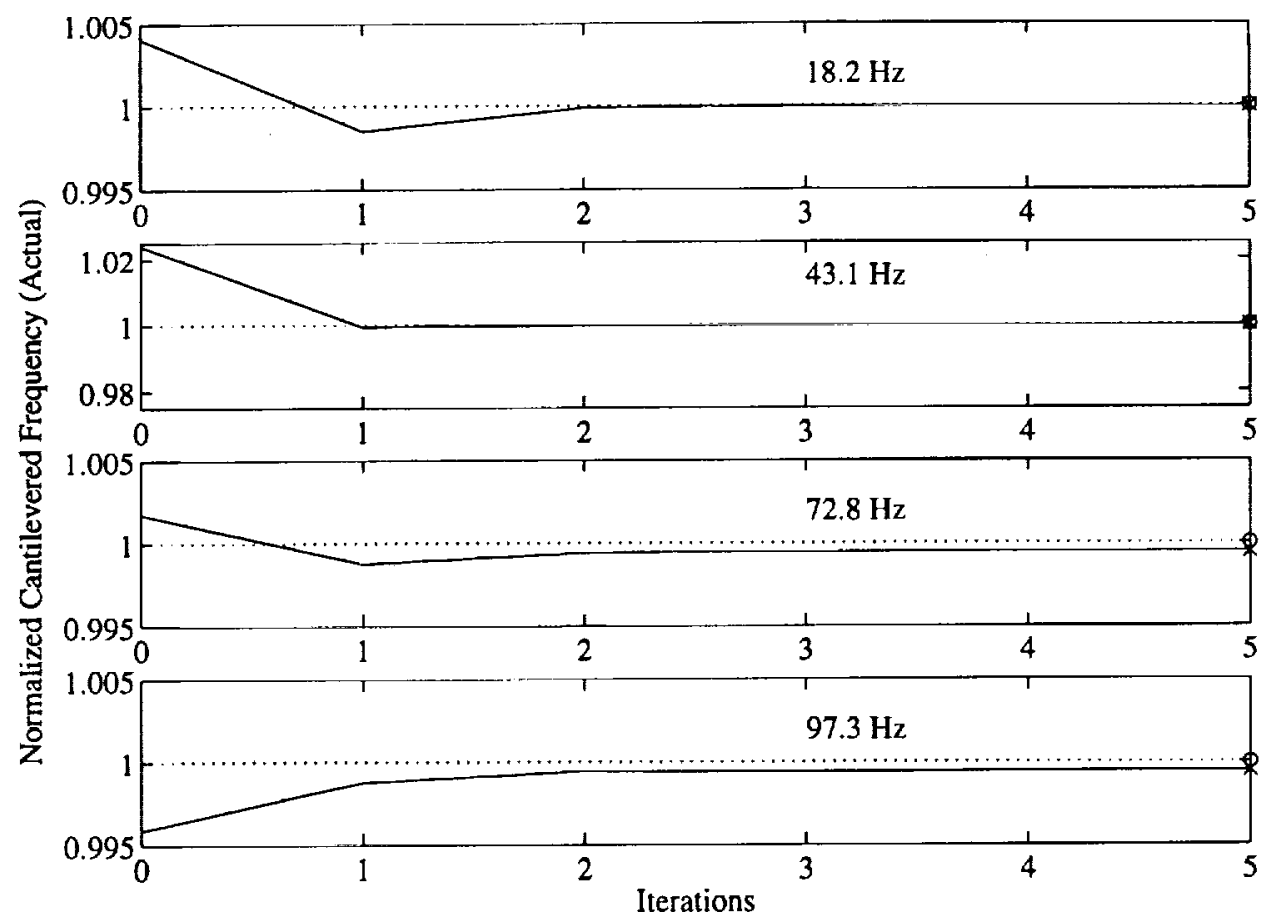

(a) Cantilevered frequencies for 5 iterations.

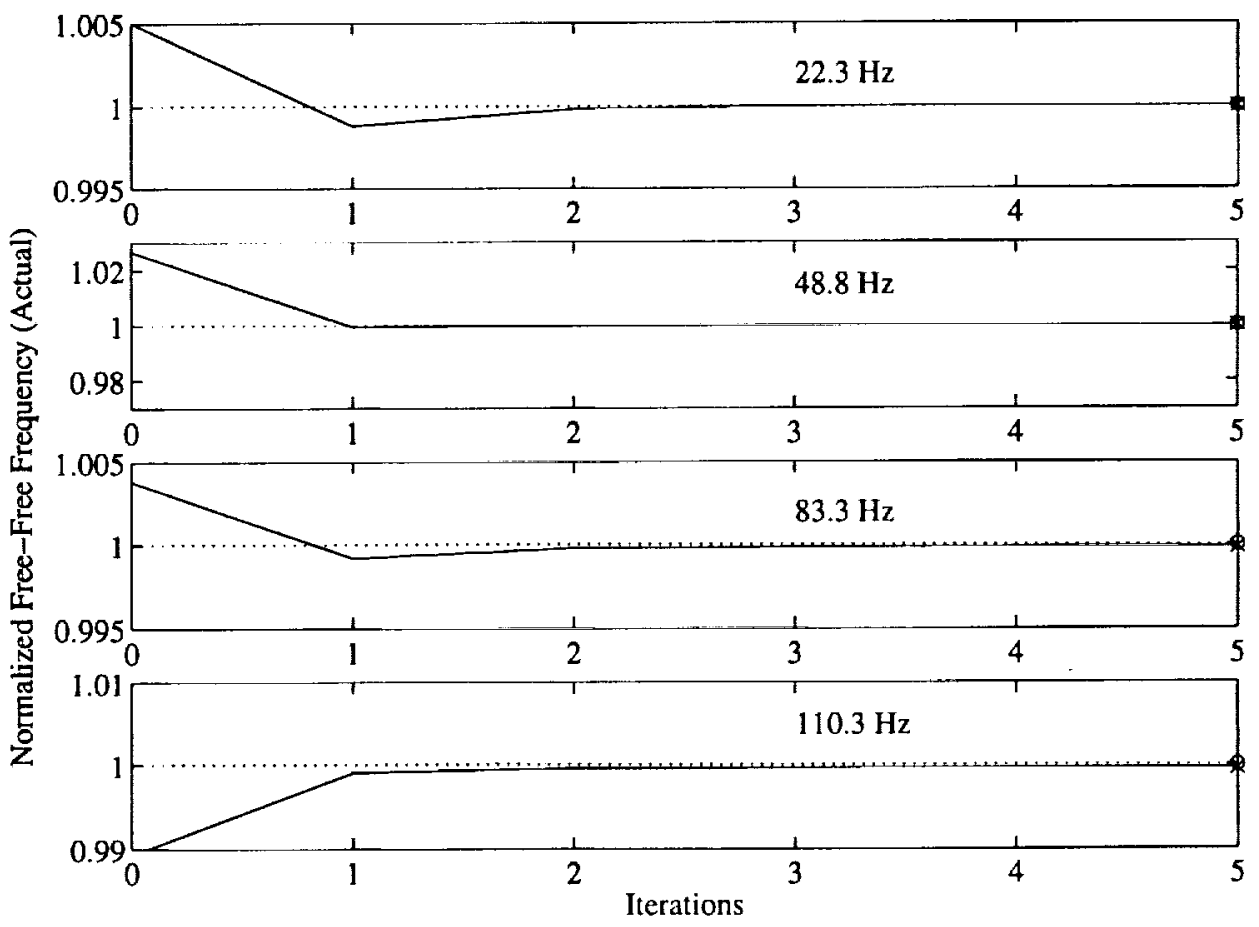

(b) Free-free frequencies for 5 iterations.

Figure 4.14: Frequency results using multiple iterations of the Sensitivity Method for Case 3 using partial measurements. (-) - updated or projected frequencies, $(x)$ - simulated mean frequency, $(o)$ actual frequency. 


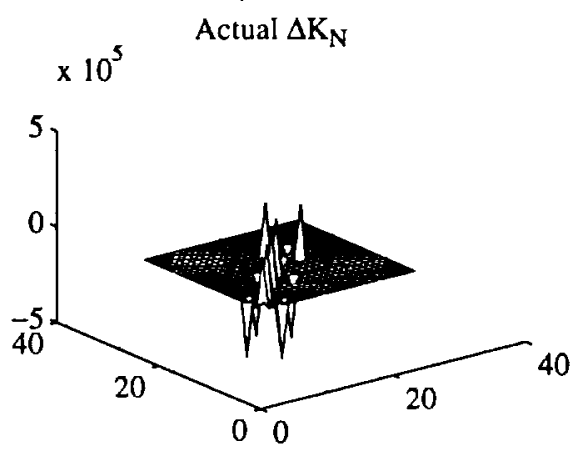

Actual $\Delta \mathrm{M}_{N}$

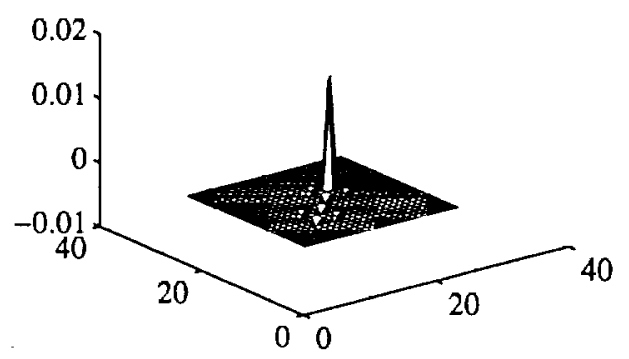

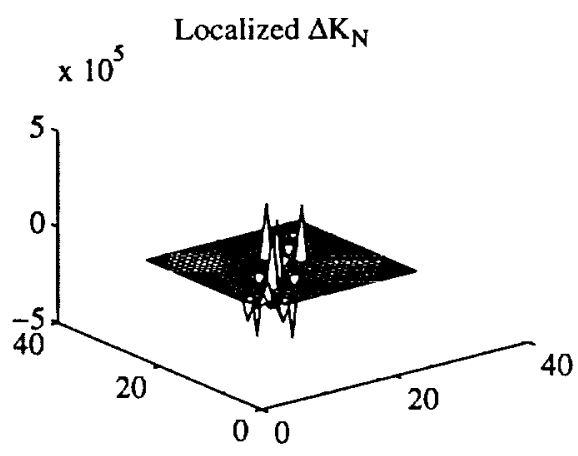

Localized $\Delta \mathrm{M}_{\mathrm{N}}$

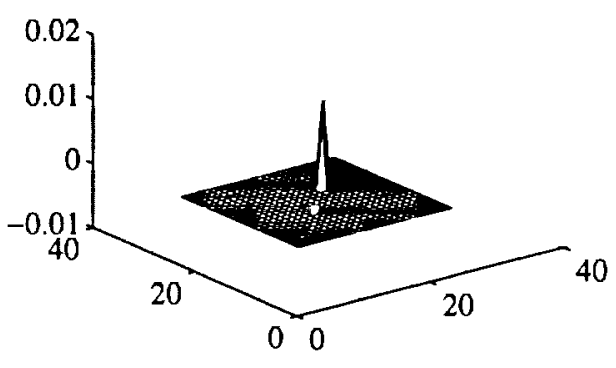

(a) Mass and stiffness mean error matrices.

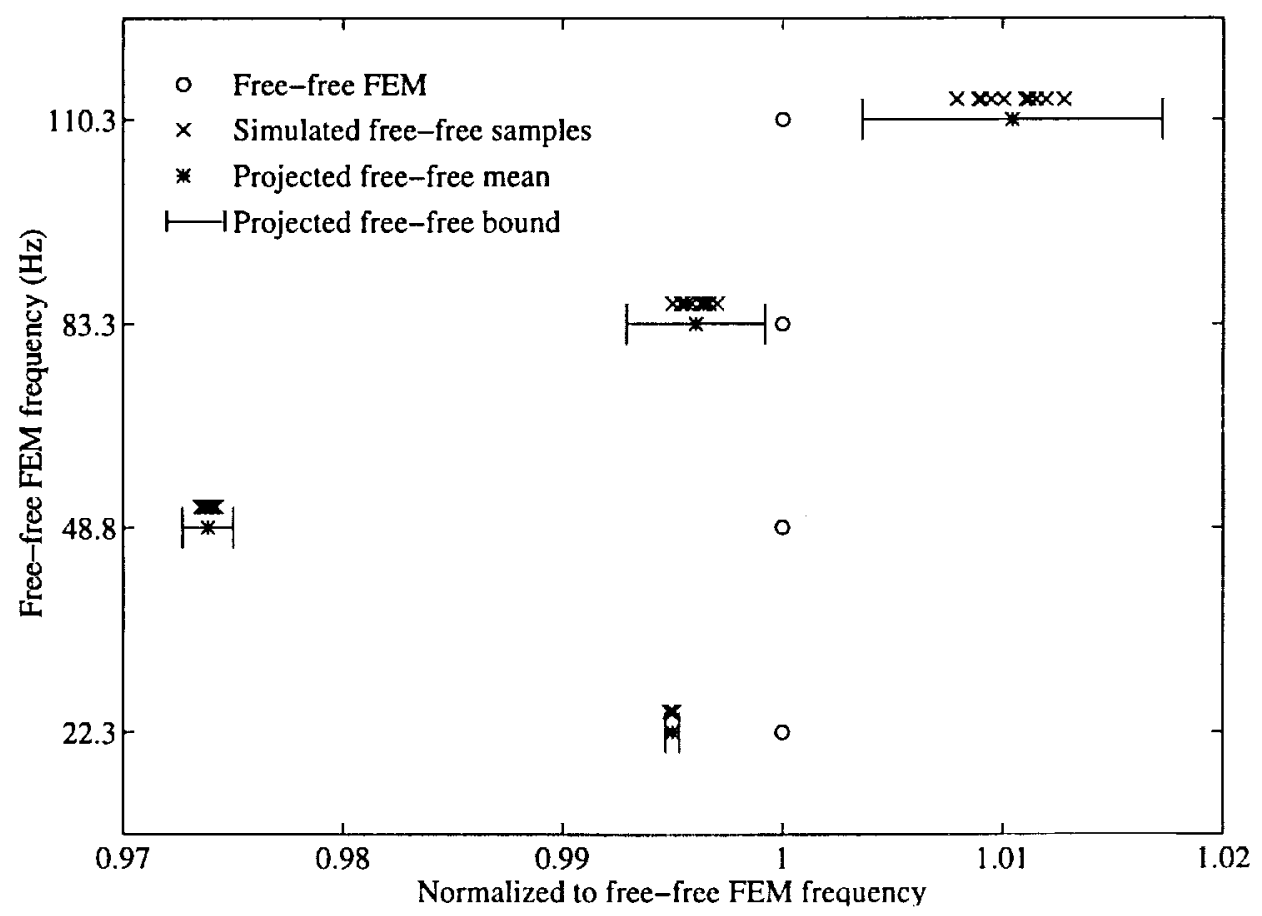

(b) Frequency results for cantilever to free-free projection of mean error and variance uncertainties.

Figure 4.15: Sensitivity Method for Case 3 using partial measurements and 5 iterations. 


\section{Chapter 5}

\section{Experimental Verification}

This chapter is used to experimentally verify the uncertainty localization and projection methods developed previously. The Middeck Active Control Experiment (MACE) is used to demonstrate the feasibility of these techniques in an experimental setting, while also showing their strengths and weaknesses. Two types of experiments are used to evaluate these methods. The first is using the MACE 1-g system as nominal to predict the modified MACE $0-\mathrm{g}$ uncertainty model. This was the primary objective of the development of these methods for the MACE flight. The second experiment utilizes a different configuration of the MACE test article. Figure 5.1 shows two configurations of the MACE test article used during the flight, termed Configuration I and II. The second experiment uses the MACE 0-g Configuration I system as nominal to predict the modified MACE $0-\mathrm{g}$ Configuration II uncertainty model. In addition to demonstrating these methods experimentally, these experiments also give insights into where improvements of the methods can be made. 


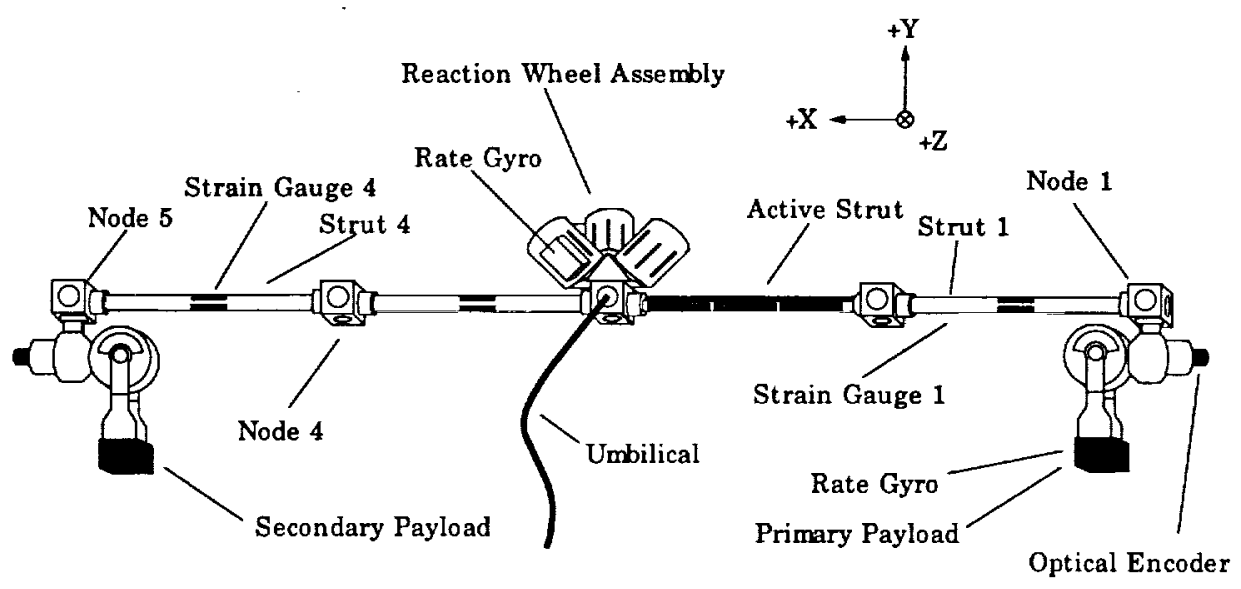

(a) MACE Configuration I

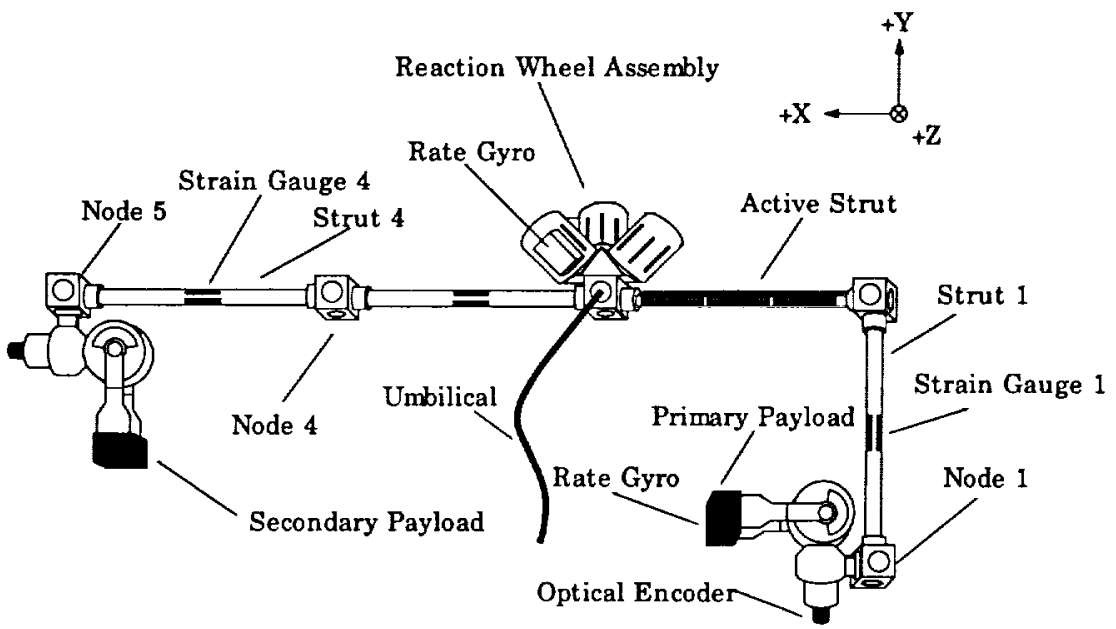

(b) MACE Configuration II

Figure 5.1: Two $0^{-} \mathrm{g}$ configurations of the MACE test article.

\subsection{MACE Configuration I $1-\mathrm{g}$ to $0-\mathrm{g}$}

The first experiment used to verify the uncertainty localization and projection methods developed in this work is MACE Configuration I 1-g, shown in Figure 2.1, to Configuration I $0-g$, shown in Figure 5.1(a). For MACE, this projection was the basis for all controllers developed prior to flight. In addition, because of the change from $1-\mathrm{g}$ to $0-\mathrm{g}$ and the reduction of overall degrees of freedom with the removal 
of the suspension system, this prediction is quite a challenging task for the methods developed in this work.

Details of the MACE Configuration I $1-\mathrm{g}$ to $0-\mathrm{g}$ uncertainty localization and projection are given in Table 5.1. The nominal system is MACE Configuration I in 1-g. The 1-g finite element model for MACE contains $n_{\mathrm{N}}=678$ degrees of freedom, of which 375 modes are retained from Nastran [66]. There are 16 data sets available for identification, producing $r_{\mathrm{N}}=17$ measured degrees of freedom $(9 \mathrm{XY}$ axes $+8 \mathrm{Z}$ axis) and $\tilde{n}_{\mathrm{N}}=24$ measured frequencies (14 XY axes $+10 \mathrm{Z}$ axis). This gives 230 measured modal uncertainties in the MACE 1-g uncertainty model (140 XY axes + $90 \mathrm{Z}$ axis). Note that this puts an upper limit on the number of macroelements used in the uncertainty localization. A summary of the MACE 1-g uncertainty model is given in Chapter 2.

The modified system is MACE Configuration I in $0-\mathrm{g}$. The $0-\mathrm{g}$ finite element model for MACE contains $n_{\mathrm{M}}=480$ degrees of freedom, of which 280 modes are retained from Nastran. There are 12 data sets available for identification, producing $r_{\mathrm{N}}=17$ measured degrees of freedom ( $9 \mathrm{XY}$ axes $+8 \mathrm{Z}$ axis) and $\tilde{n}_{\mathrm{N}}=24$ measured frequencies (14 XY axes $+10 \mathrm{Z}$ axis).

\begin{tabular}{l|c|c} 
Property & Nominal $(1-\mathrm{g})$ & Modified $(0-\mathrm{g})$ \\
\hline \hline degrees of freedom $\left(n_{(\cdot)}\right)$ & 678 & 480 \\
FEM modes retained & 375 & 280 \\
measured degrees of freedom $\left(r_{(\cdot)}\right)$ & $9 \mathrm{XY}+8 \mathrm{Z}=17$ & $9 \mathrm{XY}+8 \mathrm{Z}=17$ \\
measured frequencies $\left(\tilde{n}_{(\cdot)}\right)$ & $14 \mathrm{XY}+10 \mathrm{Z}=24$ & $8 \mathrm{XY}+6 \mathrm{Z}=14$ \\
number of data sets & 16 & 12 \\
stiffness elements & 99 & 69 \\
mass elements & 59 & 32 \\
concentrated spring elements & 9 & 0 \\
concentrated mass/inertia elements & 39 & 33
\end{tabular}

Table 5.1: MACE Configuration I 1-g to 0-g summary. 
In mapping the degrees of freedom between the two systems, one needs to examine the nominal and modified finite element models. The modified $0-\mathrm{g}$ finite element model is developed by removing the suspension degrees of freedom, and eliminating the nonlinear iterative technique to add the gravity stiffening and initial deformation. The 1-g and $0-\mathrm{g}$ finite element models are set up such that the mapping between systems given by $\eta_{\mathrm{M}}=T_{\mathrm{MN}} \eta_{\mathrm{N}}$ is

$$
T_{\mathrm{MN}}=\left[\begin{array}{ll}
I_{480} & 0_{480 \cdot 198}
\end{array}\right]
$$

For this experiment, both the matrix method and sensitivity method are used to localize and project the uncertainties. The matrix method is presented primarily because it was the method used for the MACE flight. Therefore, it was performed with no prior knowledge of the $0-\mathrm{g}$ data. It was the basis of the uncertainty model used in all of the closed loop experiments designed prior to flight. The sensitivity method, although developed after the MACE flight, is presented as an improved technique, and a better method for uncertainty localization and projection.

\subsubsection{Matrix Method}

For the MACE flight, the matrix method was used to develop an uncertainty model in $0-\mathrm{g}$. Prior to flight, however, there was minimal information available for the projection. For instance, rather than the DEKF, a technique called Frequency domain Observability Range Space Extraction (FORSE) [40], along with a Nonlinear Logarithmic Least Squares Method (NLLS) [47] were used to obtain measurement models. In addition, the measurement models were developed using the MACE system with gimbal servos. Information about normal mode shapes at the measured degrees of freedom was quite difficult to extract. Therefore, only frequency errors were localized and projected for the MACE flight.

Figure 5.2 shows the frequency results of the MACE $1-\mathrm{g}$ to $0-\mathrm{g}$ projection using the matrix method, and based only on 1-g frequency uncertainties. All model and data points are normalized by the corresponding $0-\mathrm{g}$ finite element model frequency. Note that the $0-\mathrm{g}$ data was only available after the flight. In addition, the modes 
are divided into $\mathrm{XY}$ and $\mathrm{Z}$ axes categories depending on their contribution for ease of explanation. The mean errors and bounds are exactly those used for designing controllers for the MACE flight. There are a few modes, such as $3.73,39.56$, and $49.67 \mathrm{~Hz}$ modes in which the projection works quite well. Other modes such as at 9.46, 49.18, and $56.97 \mathrm{~Hz}$ where the method seems to predict the sign of the mean error, but not the magnitude. And finally there are those modes such as at 2.26, $16.11,17.84$, and $25.03 \mathrm{~Hz}$ where the method does not even predict the sign of the mean error correctly. In examining these results, there is nothing consistent that can be concluded. This is primarily because the matrix method uses a pseudoinverse on an underdetermined system, thus giving results that may not be physically realizable. Another possible error with the matrix method is that gravity stiffening and initial deformation, which manifests themselves as a superposition of the stiffness matrix, is neglected. The matrix method cannot differentiate between physical errors in the stiffness matrix, and errors in the modeling of the gravity effects.

The matrix method was also performed using the MACE 1-g uncertainty model developed using the DEKF, including the mode shape uncertainties. The frequency results for this case are shown in Figure 5.3. The results for this case are similar to those when only eigenvector uncertainties are used, shown in Figure 5.2, and again quite inconclusive.

\subsubsection{Sensitivity Method}

After the MACE flight, the sensitivity method was developed in an attempt to rectify the inconsistencies of the matrix method, and develop the best method possible for uncertainty localization and projection, given all of the uncertainty information. The use of the sensitivity method therefore has the benefit of using mode shape, as well as frequency uncertainty data. Although this seems to disable the comparison of the methods, it should be noted that the matrix method is presented as the results prior to flight, while the sensitivity method is presented as the best technique for uncertainty localization and projection.

In developing the nominal 1-g finite element model, there are 99 stiffness elements, 


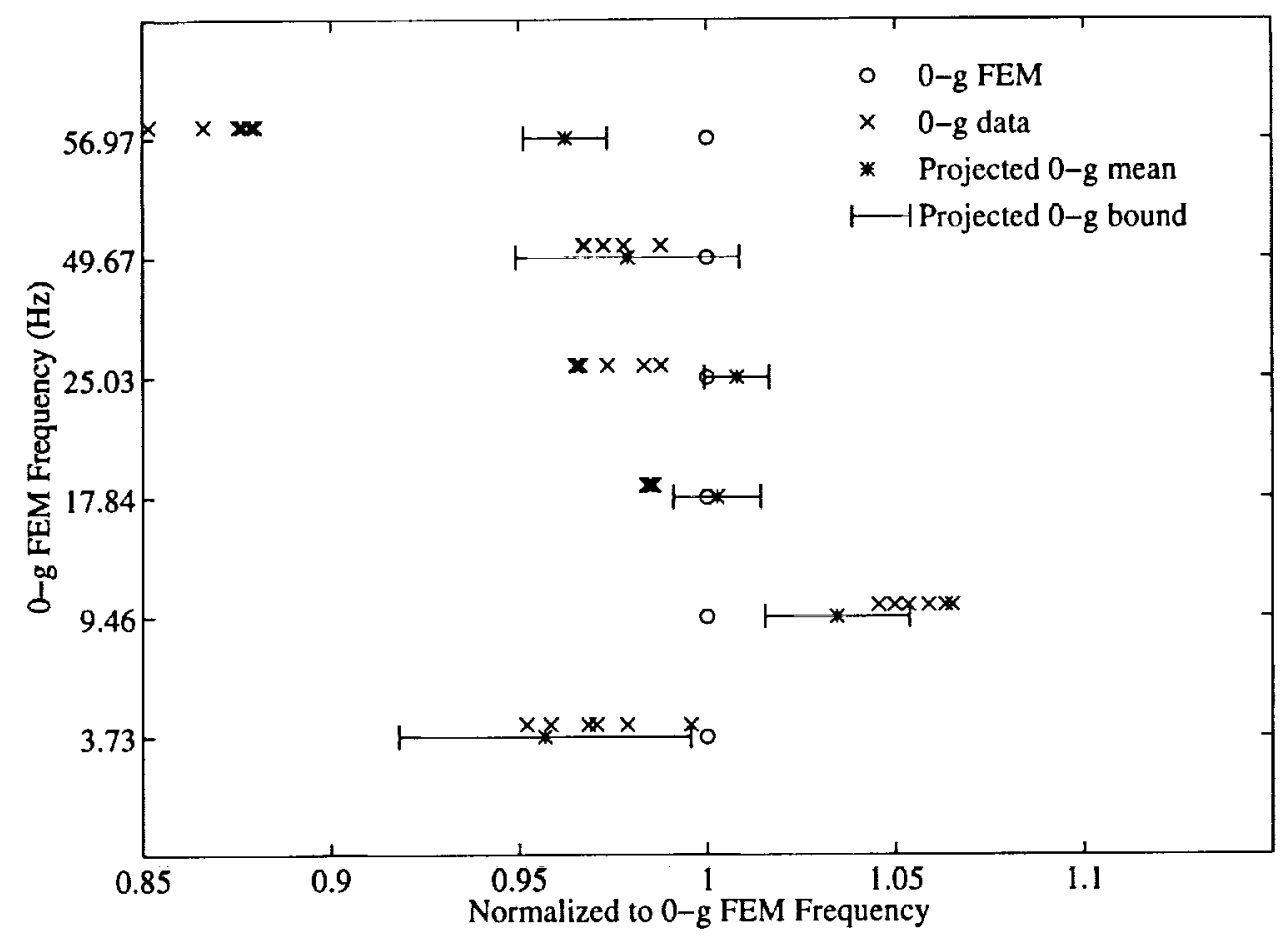

(a) XY-axes projection.

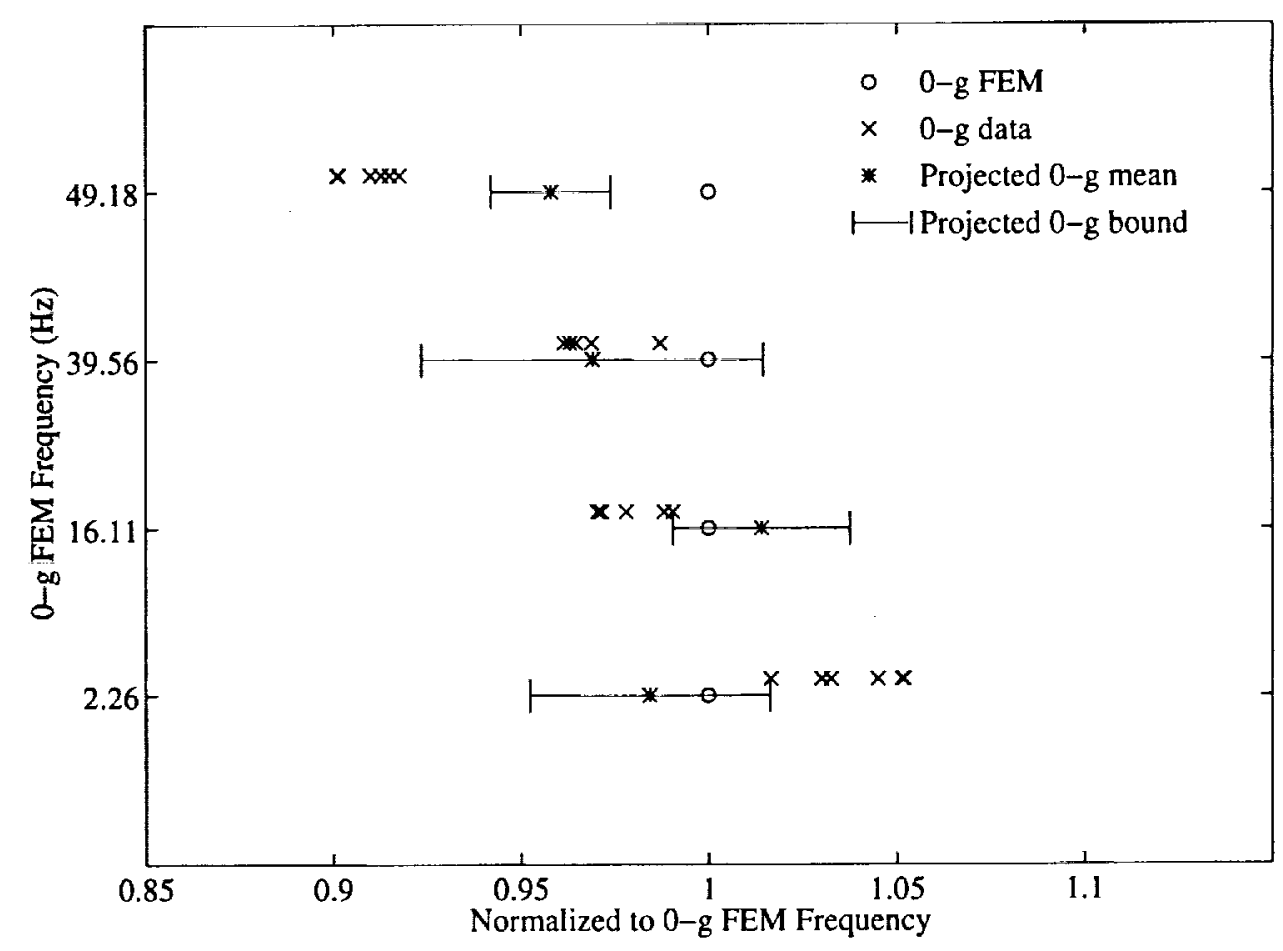

(b) Z-axis projection.

Figure 5.2: MACE Configuration I $1-\mathrm{g}$ to $0-\mathrm{g}$ projection using the matrix method with eigenvalue uncertainties. 


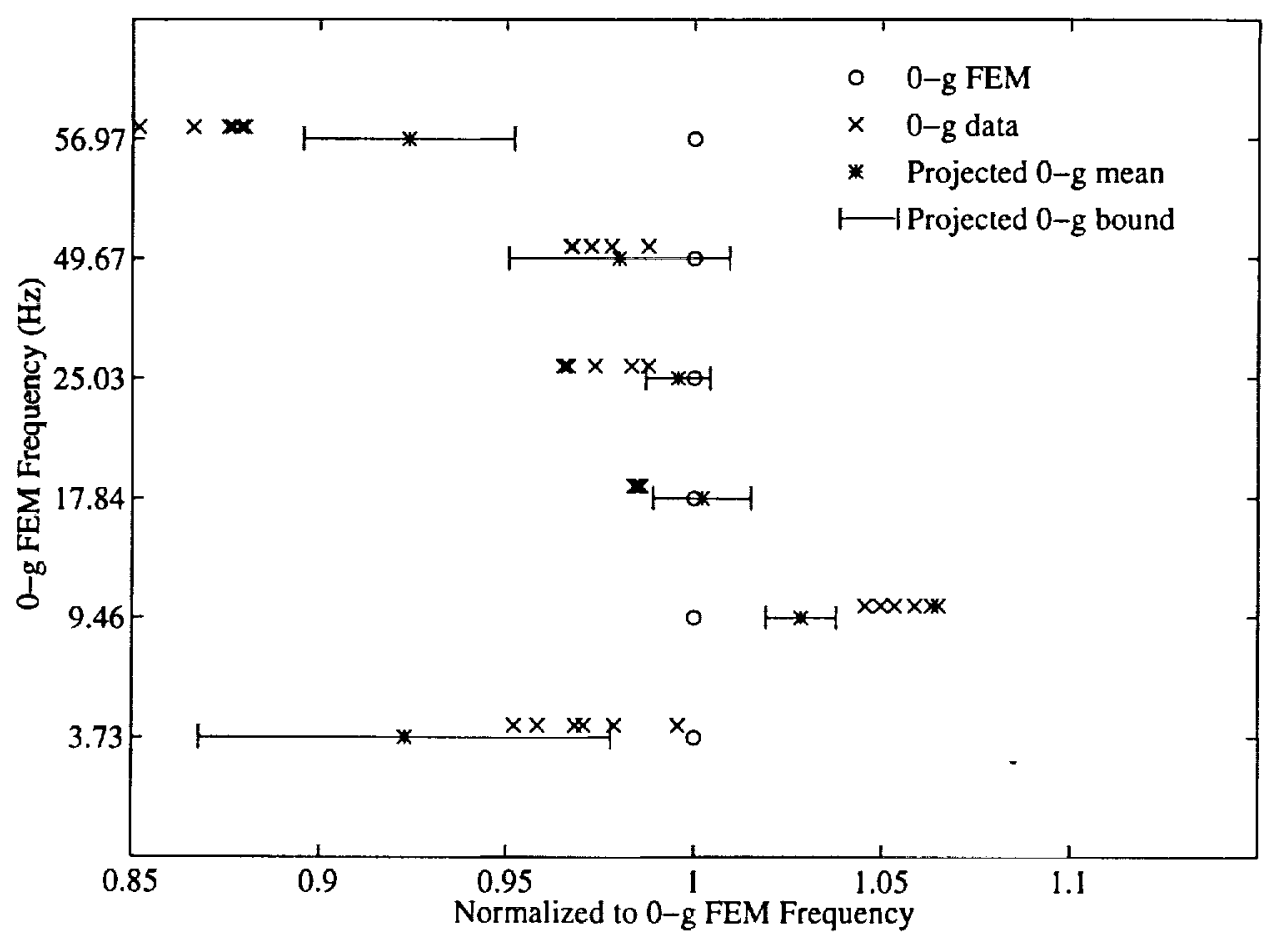

(a) XY-axes projection.

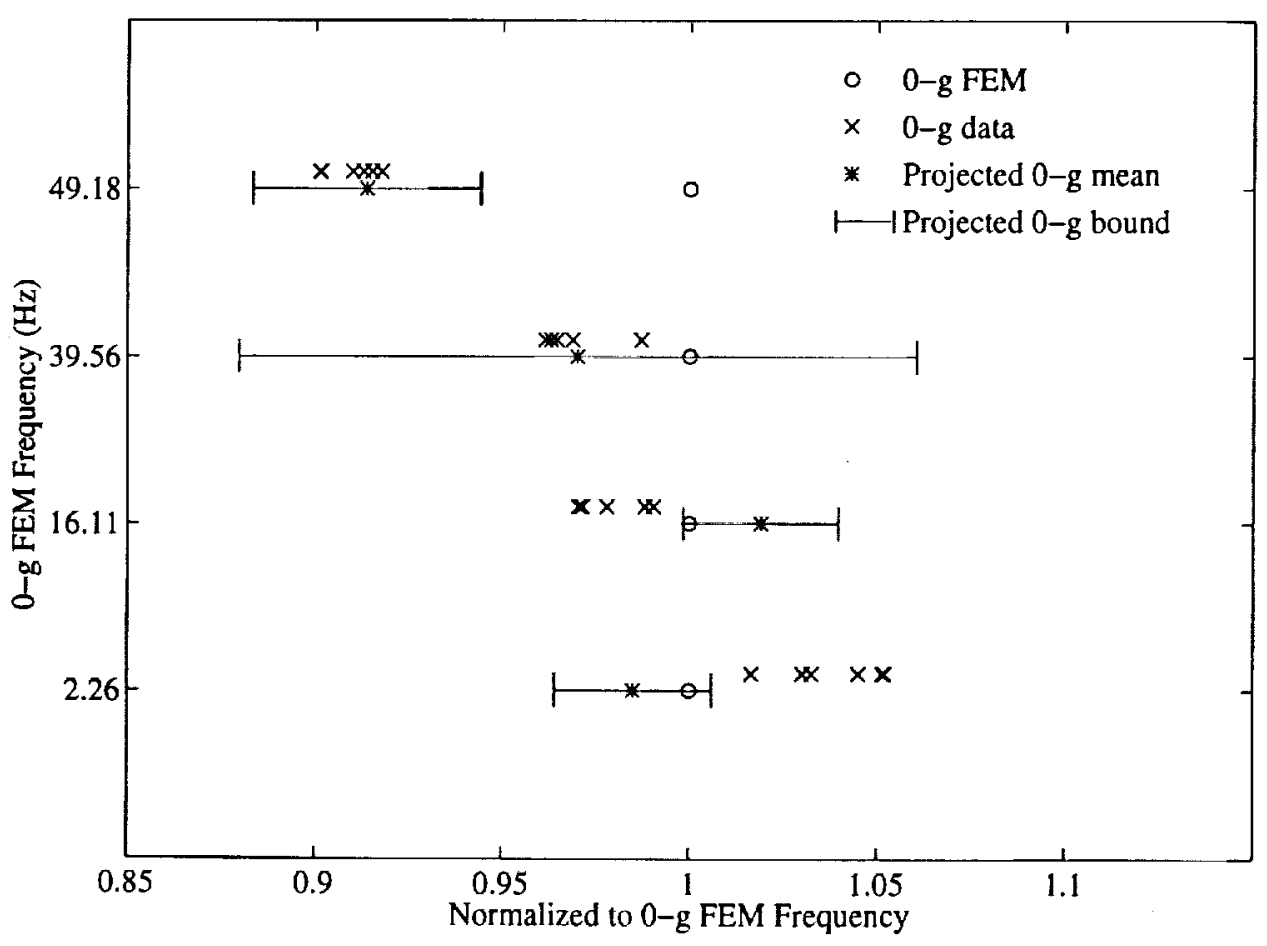

(b) Z-axis projection.

Figure 5.3: MACE Configuration I $1-\mathrm{g}$ to $0-\mathrm{g}$ projection using the matrix method with eigenvalue and eigenvector uncertainties. 
59 mass elements, 9 concentrated spring elements, and 39 concentrated inertia elements, for a total of 206. Because there are 230 measured uncertainties in the nominal system, only two steps of uncertainty localization are needed: at the element level, and at the sub-element level.

Uncertainty localization at the sub-element level again requires an examination of the element. Table 2.4 shows the six physical uncertainties that can be used in developing macroelements at the sub-element level. For this experiment, only the physical uncertainties $\Delta E, \Delta \rho$, and $\Delta L$ are used, giving two independent mass and two independent stiffness sub-elements. The physical uncertainty $\Delta t$ can be found by using a combination of the sub-elements already used; $\Delta \nu$ is assumed to be negligible. The physical uncertainty $\Delta r$ is very similar to $\Delta E$ and $\Delta \rho$. The only difference is in the torsional and rod degrees of freedom. The modes that are identified are bending, and the degrees of freedom measured are rotational. It therefore assumed that the physical uncertainty $\Delta r$ can be reproduced using a combination of $\Delta E$ and $\Delta \rho$ macroelements with only minor errors introduced.

In addition to the physical uncertainties, gravity macroelements are also defined. In Chapter 2 , the gravity effects presented are gravity stiffening and initial deformation. One can examine the nonlinear procedure from Nastran to find the type and magnitude of the gravity effects. Also, one can examine the gravity stiffening and initial deformation adjustments given in Equations 2.5-2.8. It was found that the primary gravity effects are: gravity stiffening and initial sag in the Lexan beam elements; gravity stiffening in the gimbals; and gravity stiffening in the suspensions cables. These gravity effects are also used in the development of macroelements at the sub-element level.

A two step uncertainty localization procedure was employed. Note that at at each of the two steps, a reduction in the macroelements is performed. Figure 5.4 is a pictorial representation of the location of the errors in stiffness matrix for MACE in 1-g. This was formed by examining the macroelements that are retained in the two step localization procedure. Note that this figure only gives the location of the errors retained, and not the magnitude. The axes on the left and top (given a square 
stiffness matrix) correspond to different degrees of freedom, where the abbreviations are given as follows:

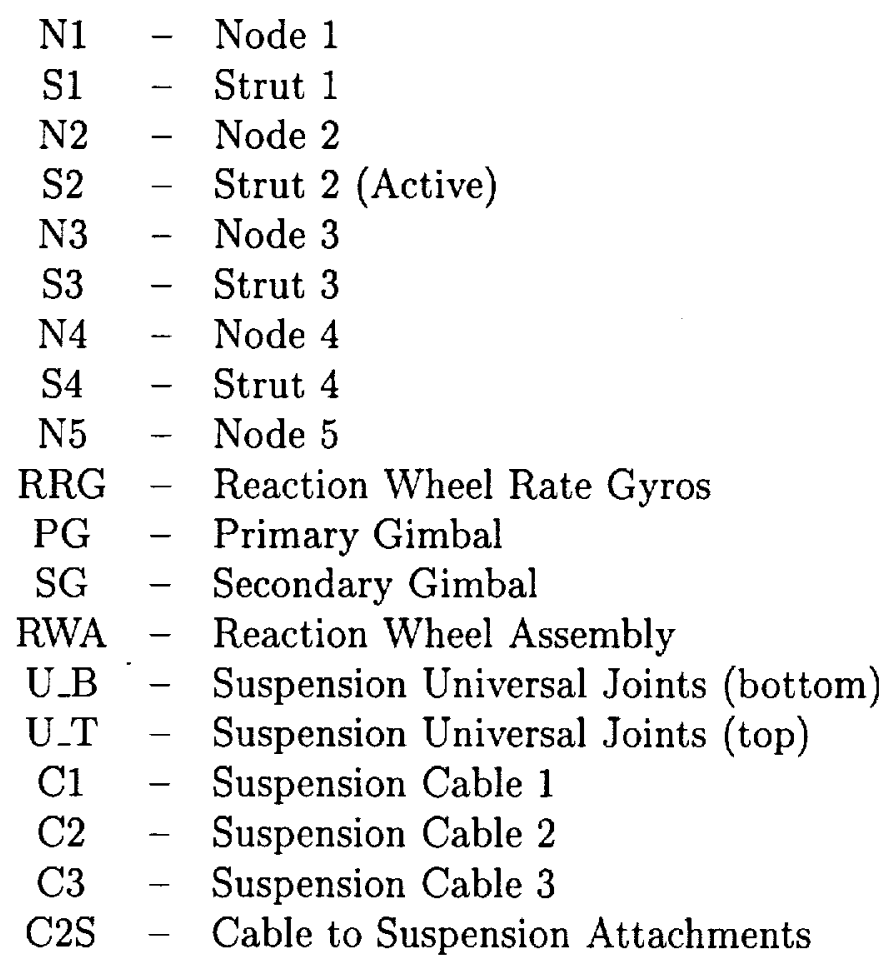

In addition, there are dotted lines in the figures to aid in the location of the errors. For instance, the first vertical and horizontal dotted lines separates node 1 from strut 1 ; the second lines separate strut 1 from node 2 , and so on.

Figure 5.4(a) shows the localized mean errors of the stiffness matrix that pertain to the physical test article. It is not surprising that most of the stiffness uncertainties in the test article shown in Figure 5.4(a) are localized to the struts. This is because the measured modes are flexible modes which are primarily bending in the struts. The types of physical uncertainties are a combination of changes in the modulus $\Delta E$ and length $\Delta L$. It is difficult to gain insight into such a large system such as this. However, general trends are that there are modulus errors at the ends of the struts, where there is a larger radius. This may indicate an error in radius (i.e. inertia). In addition, there are both length and modulus errors in the active strut. This area is likely to be the source of modeling errors because the different materials being used are modeled as a composite material. And finally, the lengths of the middle of the 


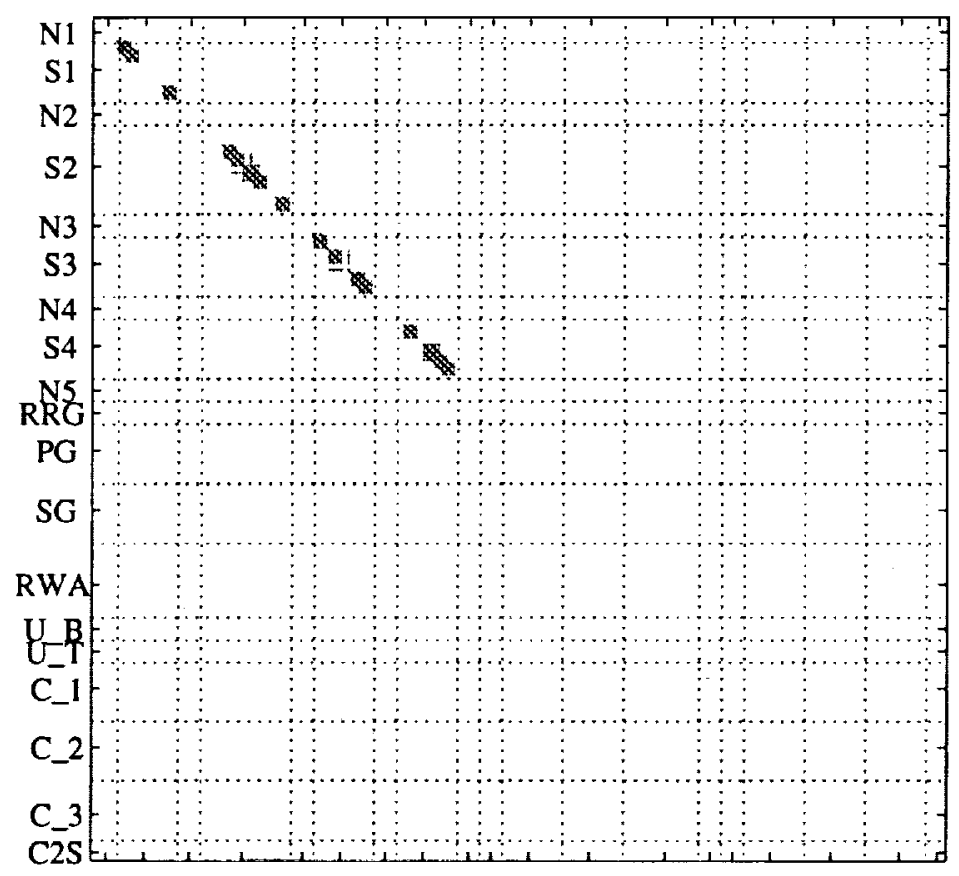

(a) Localized test article mean error uncertainties.

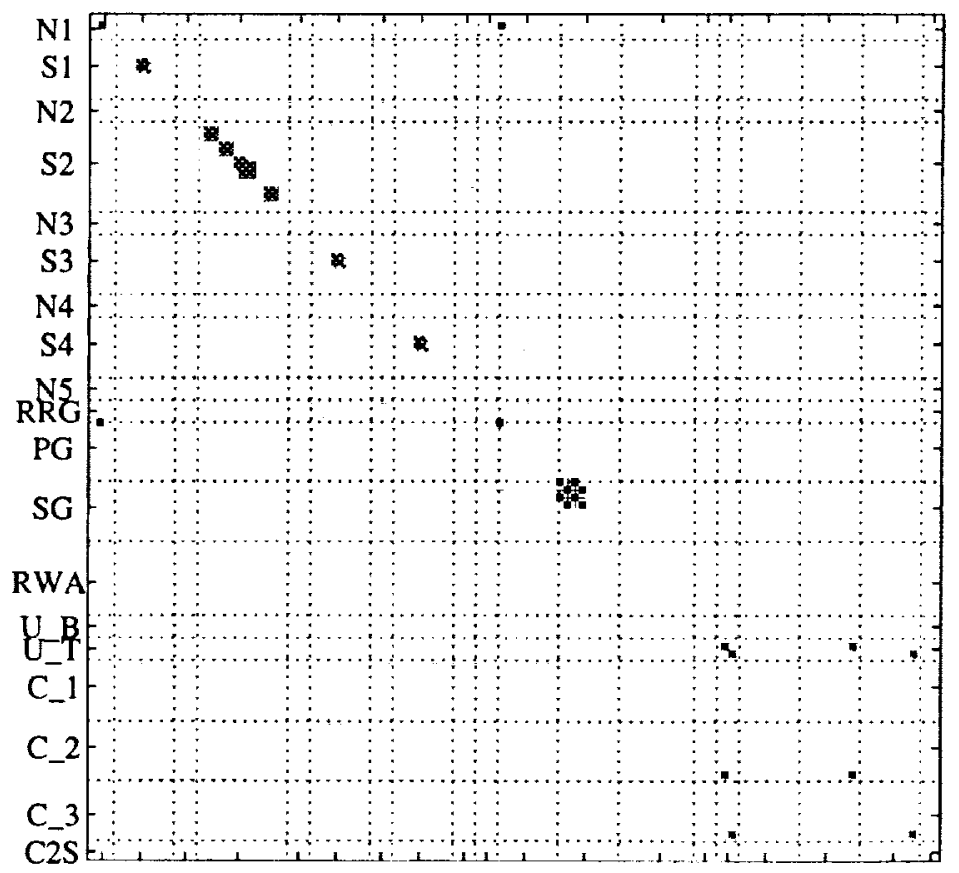

(b) Localized gravity modeling/suspension mean error uncertainties.

Figure 5.4: MACE 1-g localized stiffness matrix mean error uncertainties. 
struts, i.e. where the strain gauges are located, also are occasionally in error.

The localized errors in the modeling of gravity effects are shown in Figure 5.4(b). These uncertainties are primarily localized to the active strut area and the secondary gimbal. The secondary gimbal area makes sense because four of the identified modes are gimbal pendular modes, which are stiffened with the Nastran stiffening adjustment. The active strut area is more difficult to interpret, however. Although it makes sense for this area to contain large errors, it does not make sense that these errors are primarily due to gravity. It may be that this area contains large errors, and sensitivity method has trouble making the distinction between errors in the gravity adjustment, and errors in the physical parameters. In addition, the center element of each strut where the strain gauge acts appears to be the source of gravity modeling errors.

A final gravity adjustment that seems to be in error is in the suspension cables. Because there are very large gravity effects captured in the tensioning of the suspension cables, intuitively there should be a lot of modeling errors in this area. However, this is not the case, as shown in Figure 5.4(b). This may be a result of the lack of measured suspension information, i.e. few measured suspension modes and degrees of freedom.

In addition to stiffness uncertainties, the sensitivity method also localizes mean errors in the mass matrix. Figure 5.5 shows the localized mass errors. Notice that there are very few mass uncertainties. This is understandable because masses and inertias are measured more easily than other parameters such as the modulus of elasticity. Figure 5.5 shows that the mass properties for MACE are modeled quite well.

Figure 5.6 shows the frequency results of the projection of both the 1-g mean error and variance uncertainties into 0-g for MACE using the sensitivity method. Notice the improvement using the sensitivity method compared to the matrix method (Figure 5.3). The prediction of mean errors and bounds is excellent for all but the 2.26 and $9.46 \mathrm{~Hz}$ modes. It is thought that these modes are difficult to predict because of their coupling with the gravity effects.

The process of localizing uncertainties for the MACE system was quite a difficult 


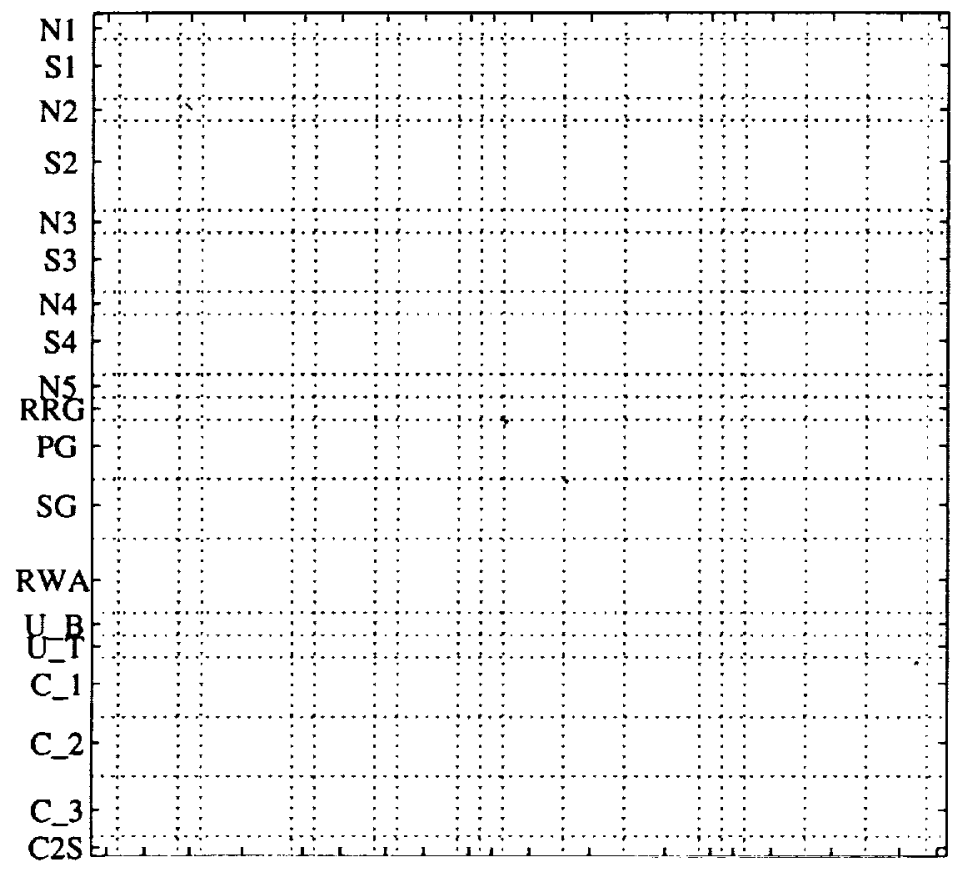

Figure 5.5: MACE 1-g localized mass matrix mean error uncertainties.

task for the MACE $1-\mathrm{g}$ to $0-\mathrm{g}$ example. It is thought that the number and type of sensors make it difficult for the sensitivity method to ascertain the difference between a physical uncertainty in an element, such as an error in the modulus, and a gravity stiffening uncertainty, such as an initial deformation. In addition, the sensitivity method (and localization and update methods in general) work best for small errors in the already defined parameters. The errors in gravity effects that are localized tended to be quite large. Table 5.2 shows a summary of the mean changes of frequencies from $1-\mathrm{g}$ to $0-\mathrm{g}$, along with the predicted change in finite element models from $1-\mathrm{g}$ to $0-\mathrm{g}$. For some modes such as $4.6 \mathrm{~Hz}$, the finite element model predicts the changes quite well. However, for modes such as $17.82,24.89$, and $56.16 \mathrm{~Hz}$, the predicted change is incorrect in sign and magnitude. This indicates difficulties in the modeling, which can only be overcome by a more thorough modeling of the gravity effects.

In order to accurately model and update modeling of gravity effects, a large number of different types of sensors must be used. Additional sensors on the nodes of the 


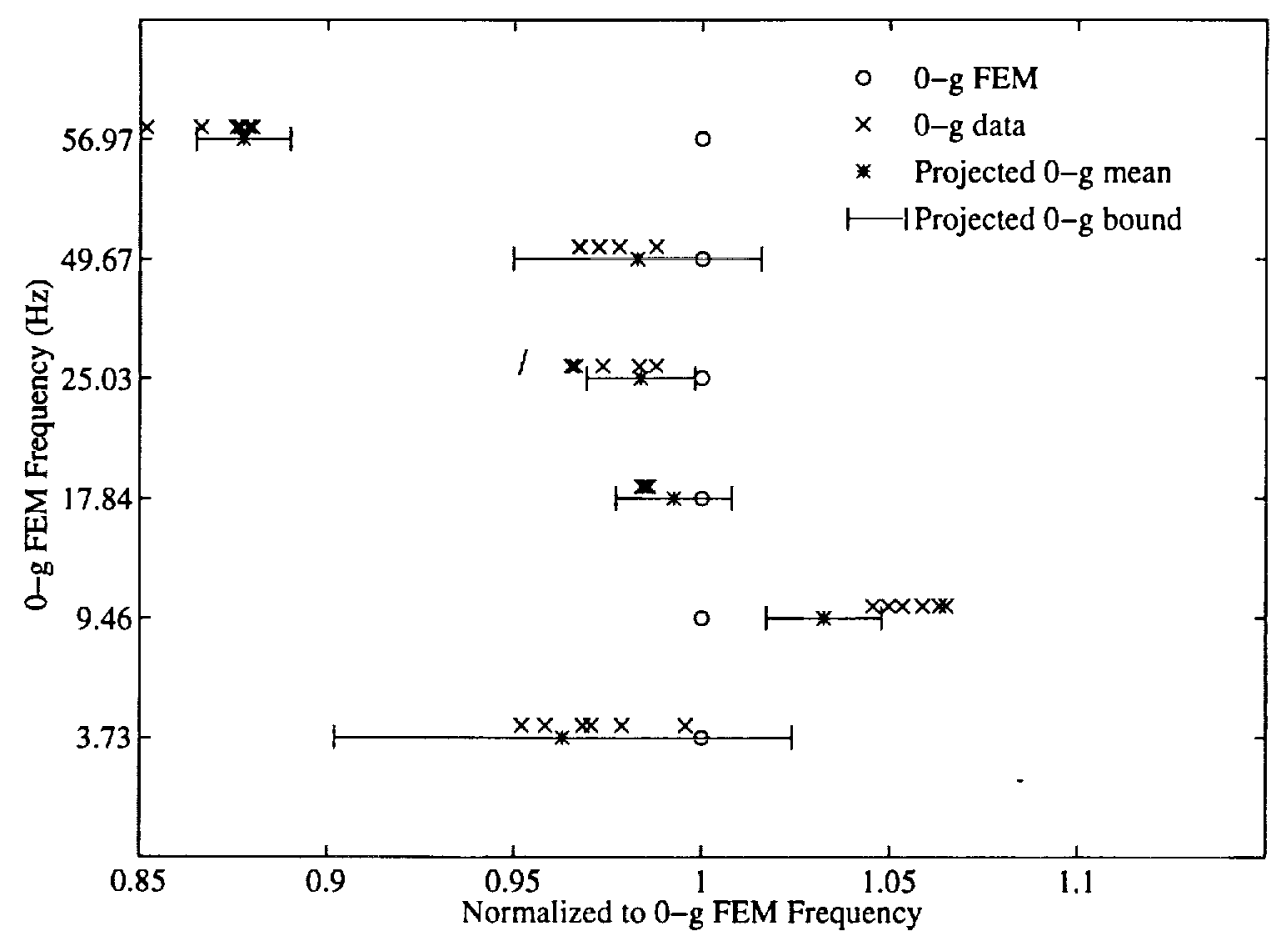

(a) $\mathrm{XY}$-axes projection.

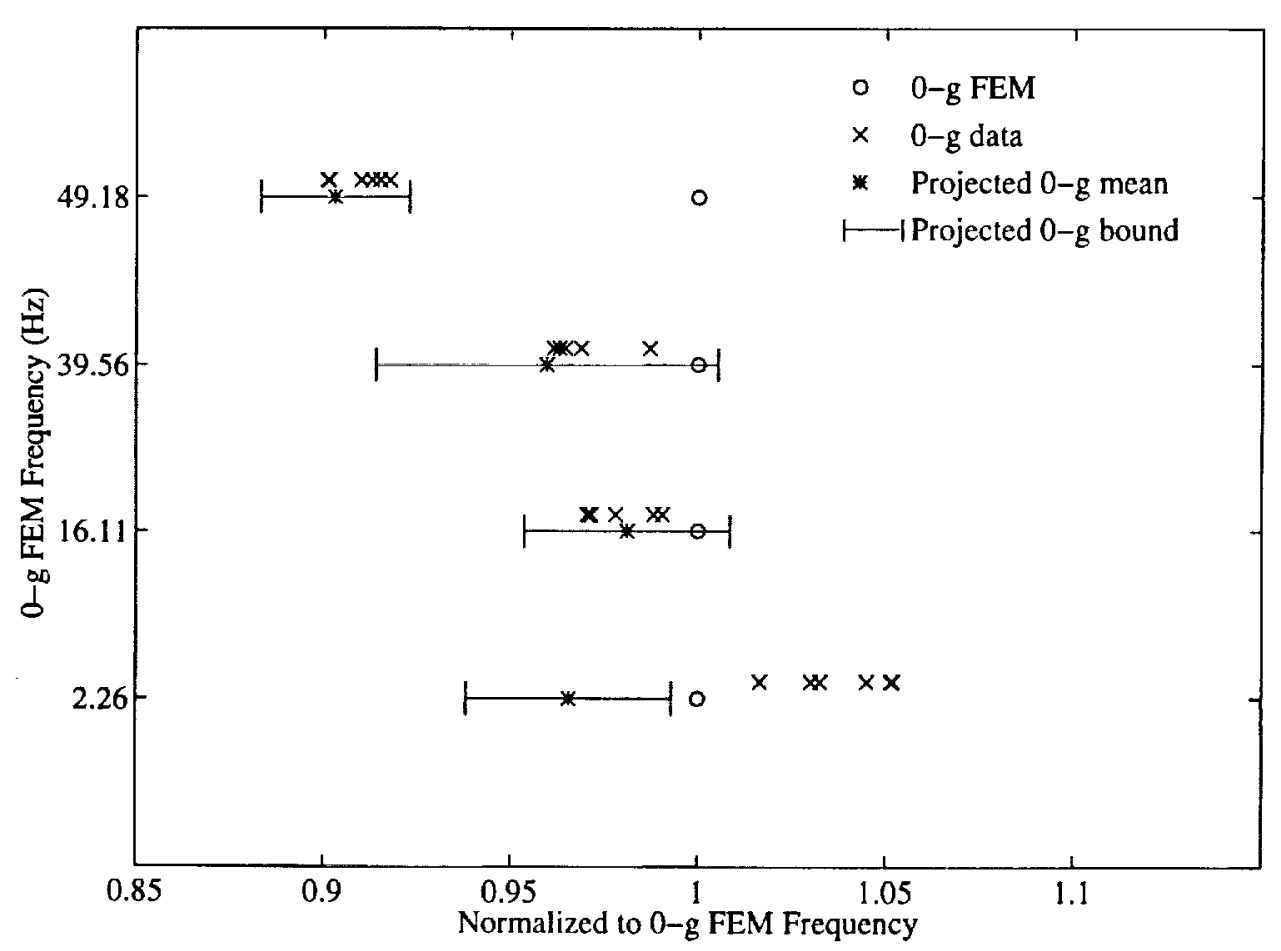

(b) Z-axis projection.

Figure 5.6: MACE Configuration I $1-\mathrm{g}$ to $0-\mathrm{g}$ projection úsing the sensitivity method. 


\begin{tabular}{|c|c|c|}
\hline 1-g Frequency (Hz) & FEM 1-g to 0-g (\%) & Data 1-g to 0-g (\%) \\
\hline \hline 2.24 & -1.1 & -7.8 \\
\hline 4.60 & 18.9 & 17.7 \\
\hline 9.79 & 3.3 & 2.4 \\
\hline 16.19 & 0.4 & 4.1 \\
\hline 17.82 & -0.1 & 1.7 \\
\hline 24.89 & -0.5 & 2.9 \\
\hline 39.41 & -0.3 & 0.0 \\
\hline 48.84 & -0.6 & 4.2 \\
\hline 49.01 & -1.3 & -0.9 \\
\hline 56.16 & -1.4 & 7.8 \\
\hline
\end{tabular}

Table 5.2: MACE $1-\mathrm{g}$ to $0-\mathrm{g}$ changes

test article and the suspension system would aid in the localization of errors in the modeling of gravity effects. In addition, more input into the modeling of the gravity effects is needed, rather than assuming that the nonlinear iterative gravity stiffening procedure in Nastran works correctly. Although is appears that this procedure works well for gravity stiffening in the cables and the gimbals, it may need additional tuning for the gravity effects within the test article that is not available.

An additional piece of work that could have been attempted in the MACE 1-g to $0-g$ example is the use of the iterative uncertainty localization strategy as shown in Figure 4.5. This more complex strategy may have improved the results for the 2.26 and $9.46 \mathrm{~Hz}$ modes. However, because of the difficulties in using a sparse eigenvalue solver that could also handle multiple rigid body modes, this option was not attempted and is left for future work.

In order to show the overall results of the uncertainty localization and projection for the sensitivity method including mode shapes uncertainties, transfer functions are plotted. Figure 5.7(a) shows the transfer function from primary $\mathrm{X}$ gimbal to primary $\mathrm{X}$ rate gyro using the $0-\mathrm{g}$ finite element model, the $0-\mathrm{g}$ finite element model shifted 


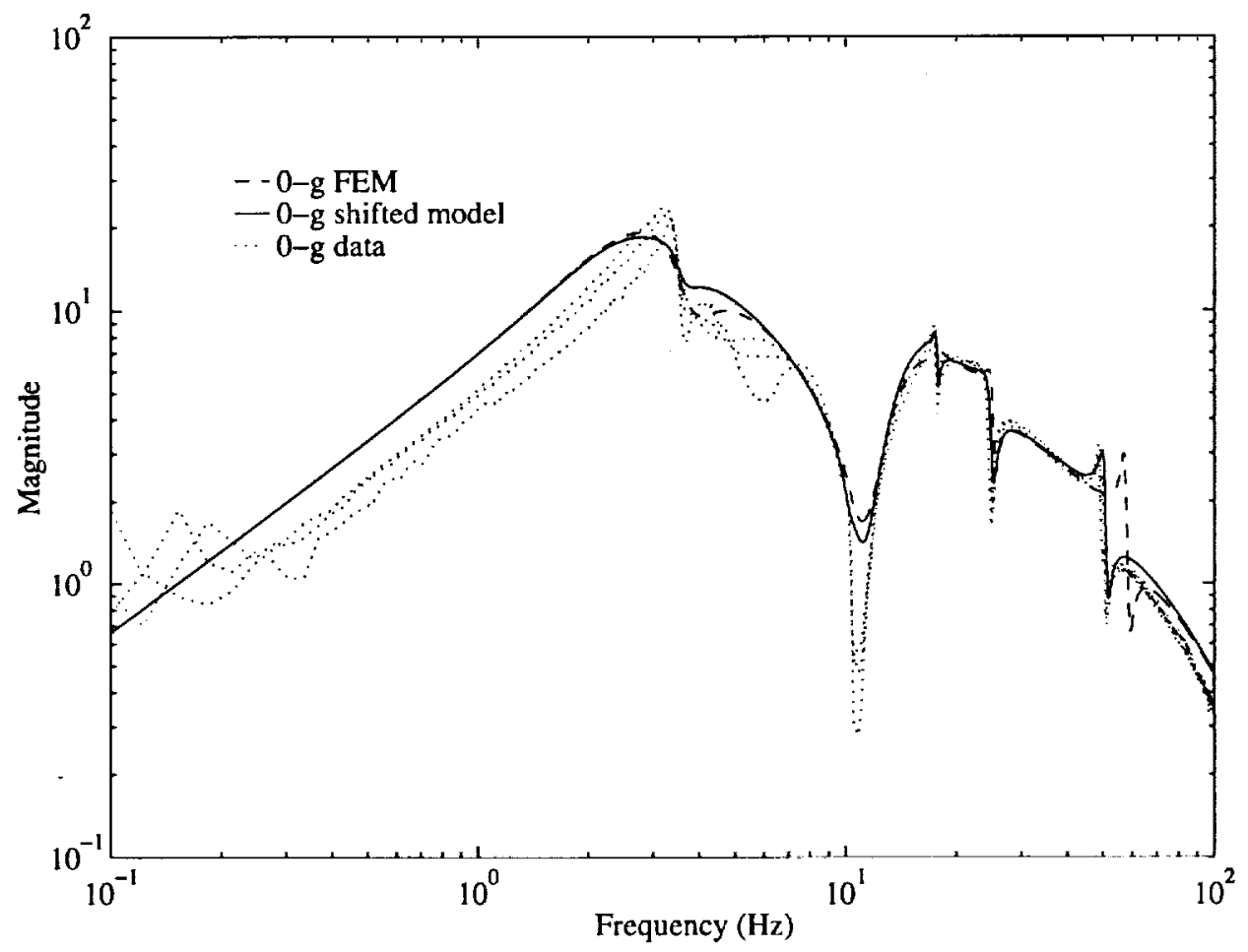

(a) Primary X Gimbal to Primary X Rate Gyro.

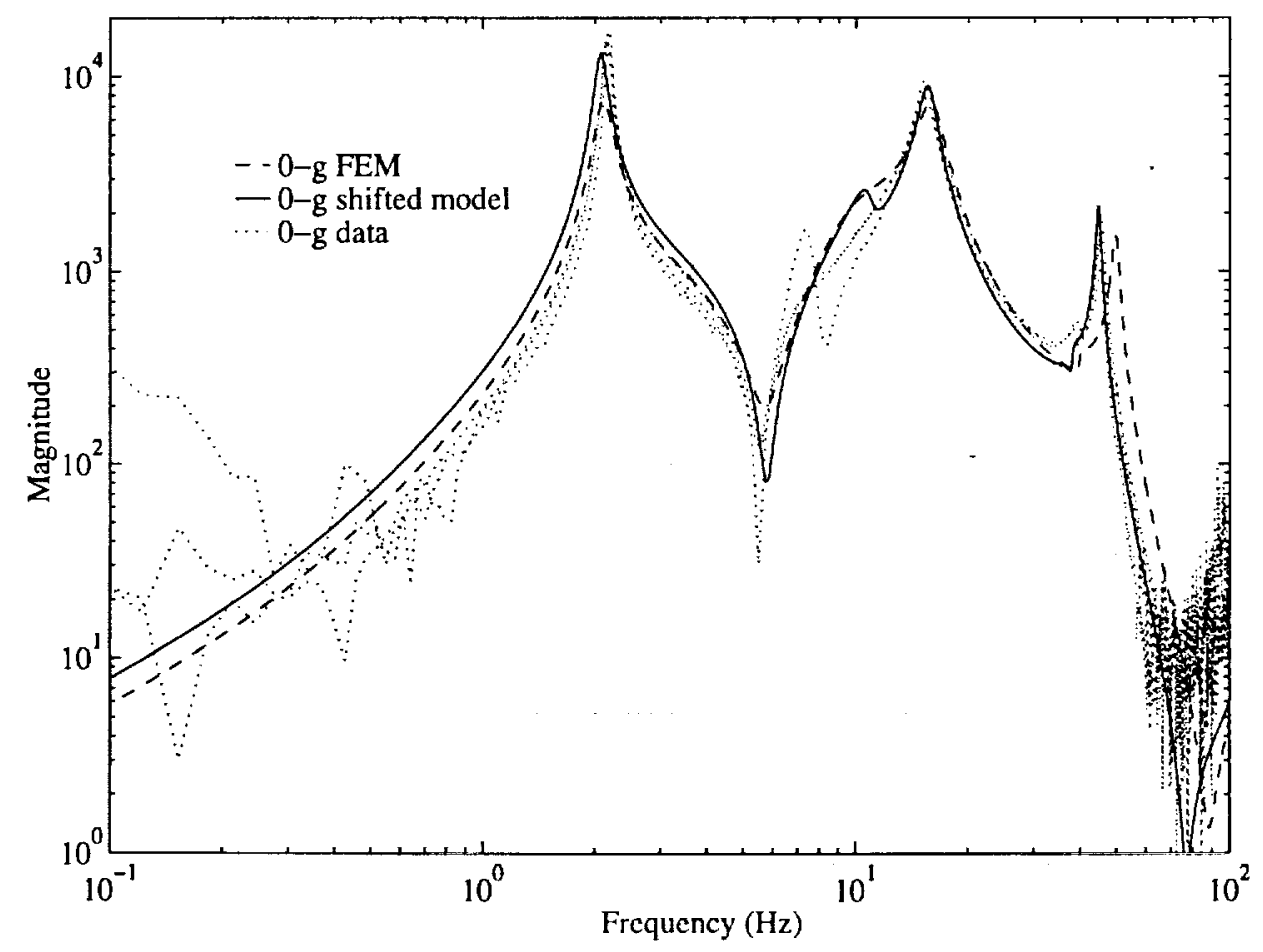

(b) Primary Z Gimbal to Strut 3 Z Strain Gauge.

Figure 5.7: MACE 0-g FEM, 0-g FEM shifted by projected mean errors, and three sets of data. 
by the projected mean errors, and three data sets taken on-orbit. Note that the 0-g damping ratios are shifted by the mean errors from the 1-g uncertainty model. Although similar, there are two important areas of improvement of the shifted model. First, at high frequency, the $56.97 \mathrm{~Hz}$ mode has been predicted correctly, while there is a $15 \%$ error in the finite element model. Secondly, the residue of $17.84 \mathrm{~Hz}$ mode in the shifted model matches that of the data, thus changing the sign of the finite element model residue.

Similar improvements can be seen in Figure 5.7(b), which plots primary Z gimbal to strut 3 strain gauge. At high frequency, the $49.18 \mathrm{~Hz}$ mode has been predicted correctly; and the zero at approximately $6 \mathrm{~Hz}$ is more lightly damped, thus more accurately matching the data. Note also the small error in the prediction of the 2.24 $\mathrm{Hz}$ mode resulting from the incorrect prediction in the projected $0-\mathrm{g}$ uncertainty model.

The three $0-\mathrm{g}$ transfer functions shown in Figure 5.7 are taken from identification experiments using three different amplitude levels. Notice in Figure 5.7(b) there is a small resonance that occurs in one of the transfer functions. This indicates the structure possibly contains stronger nonlinearities than first suspected. Therefore, another possible source of error in the uncertainty localization and projection process is strong nonlinearities in the system.

\subsection{MACE 0-g Configuration I to Configuration II}

The second experiment used to verify the uncertainty localization and projection methods developed in this work is MACE 0-g Configuration I, shown in Figure 5.1(a), to 0-g Configuration II, shown in Figure 5.1(b). This example is much simpler than the previous 1-g to $0-\mathrm{g}$ projection because there are no modeling of gravity effects or suspension degrees of freedom.

Details of the MACE $0-\mathrm{g}$ Configuration I to $0-\mathrm{g}$ Configuration II uncertainty localization and projection are given in Table 5.3. The nominal system is MACE Configuration I in $0-\mathrm{g}$. The $0-\mathrm{g}$ finite element model for MACE contains $n_{\mathrm{M}}=480$ 


\begin{tabular}{l|c|c} 
Property & Nominal $(0-\mathrm{g} \mathrm{C1})$ & Modified (0-g C2) \\
\hline \hline degrees of freedom $\left(n_{(\cdot)}\right)$ & 480 & 480 \\
FEM modes retained & 280 & 280 \\
measured degrees of freedom $\left(r_{(\cdot)}\right)$ & $9 \mathrm{XY}+8 \mathrm{Z}=17$ & $9 \mathrm{XY}+8 \mathrm{Z}=17$ \\
measured frequencies $\left(\tilde{n}_{(\cdot)}\right)$ & $8 \mathrm{XY}+6 \mathrm{Z}=14$ & $8 \mathrm{XY}+6 \mathrm{Z}=14$ \\
number of data sets & 12 & 4 \\
stiffness elements & 69 & 69 \\
mass elements & 32 & 32 \\
concentrated spring elements & 0 & 33 \\
concentrated mass/inertia elements & 33 & 0
\end{tabular}

Table 5.3: MACE $0-\mathrm{g}$ Configuration I to Configuration II summary.

degrees of freedom, of which 280 modes are retained from Nastran. There are 12 data sets available for identification, producing $r_{\mathrm{N}}=17$ measured degrees of freedom $(9 \mathrm{XY}$ axes $+8 \mathrm{Z}$ axis) and $\tilde{n}_{\mathrm{N}}=14$ measured frequencies ( $8 \mathrm{XY}$ axes $+6 \mathrm{Z}$ axis). This gives 134 measured modal uncertainties in the MACE $0-\mathrm{g}$ uncertainty model (80 XY axes $+54 \mathrm{Z}$ axis). Note that this puts an upper limit on the number of macroelements used in the uncertainty localization. The MACE $0-\mathrm{g}$ uncertainty model was developed in an analogous manner to the 1-g uncertainty model presented in Chapter 2.

The modified system is MACE Configuration II in $0^{-} \mathrm{g}$. The $0^{-} \mathrm{g}$ finite element model for the second configuration of MACE contains $n_{\mathrm{M}}=480$ degrees of freedom, of which 280 modes are retained from Nastran. There are 12 data sets available for identification, producing $r_{\mathrm{N}}=17$ measured degrees of freedom ( $9 \mathrm{XY}$ axes $+8 \mathrm{Z}$ axis) and $\tilde{n}_{\mathrm{N}}=14$ measured frequencies ( $8 \mathrm{XY}$ axes $+6 \mathrm{Z}$ axis). Notice the similarities between the two systems, as shown in Table 5.3.

In mapping the degrees of freedom between the two systems, one needs to examine the Configuration I and Configuration II finite element models. The Configuration II finite element model is developed by rotating the corresponding degrees of freedom that have been turned down in the "L" shape. This rotation matrix for each node is 
given by

$$
T_{c_{2} \cdot c_{1}}=\left[\begin{array}{cccccc}
0 & 1 & 0 & & & \\
-1 & 0 & 0 & & 0 & \\
0 & 0 & 1 & & & \\
& & & 0 & 1 & 0 \\
& 0 & & -1 & 0 & 0 \\
& & & 0 & 0 & 1
\end{array}\right]
$$

By mapping the other degrees of freedom as one to one, the modified to nominal, or Configuration II to Configuration I mapping $T_{\mathrm{MN}}$ can be developed.

Figure 5.8 shows the localized mass and stiffness mean error matrices for the $0-\mathrm{g}$ uncertainty model. Although it is difficult to compare to those localized in 1-g because there is no insight into the magnitude of the errors, there are a few similarities. First, the mass contribution is again quite small. This again shows how well the masses/inertias are modeled for MACE. In addition, the active strut degrees of freedom again were the subject of large errors, as were the ends of each of the passive struts. Overall, the comparison is fairly good.

Figure 5.9 shows the frequency results of the MACE 0-g Configuration I to Configuration II projection. Notice how most of the Configuration II data points lie within the mean errors and bounds predicted using the uncertainty localization and projection methods. The only points which do not $(8.41$ and $17.60 \mathrm{~Hz})$ have only one point that is not within the predicted ranges, and in each case, the parameter estimate had not converged in the DEKF. With more time domain data, these estimates would be within the predicted ranges as well. Application of the methods to this example was much simpler, and easier to do compared to the 1-g to $0-\mathrm{g}$ projection because of the elimination of the gravity effects and suspension degrees of freedom.

Table 5.4 shows the change between the Configuration I and Configuration II frequencies of the finite element model and data. Notice that although the magnitude is not always correct, the general trends are very good. This shows that the overall modeling of the MACE test article is very good, which makes it quite easy to localize and project the remaining small uncertainties. 


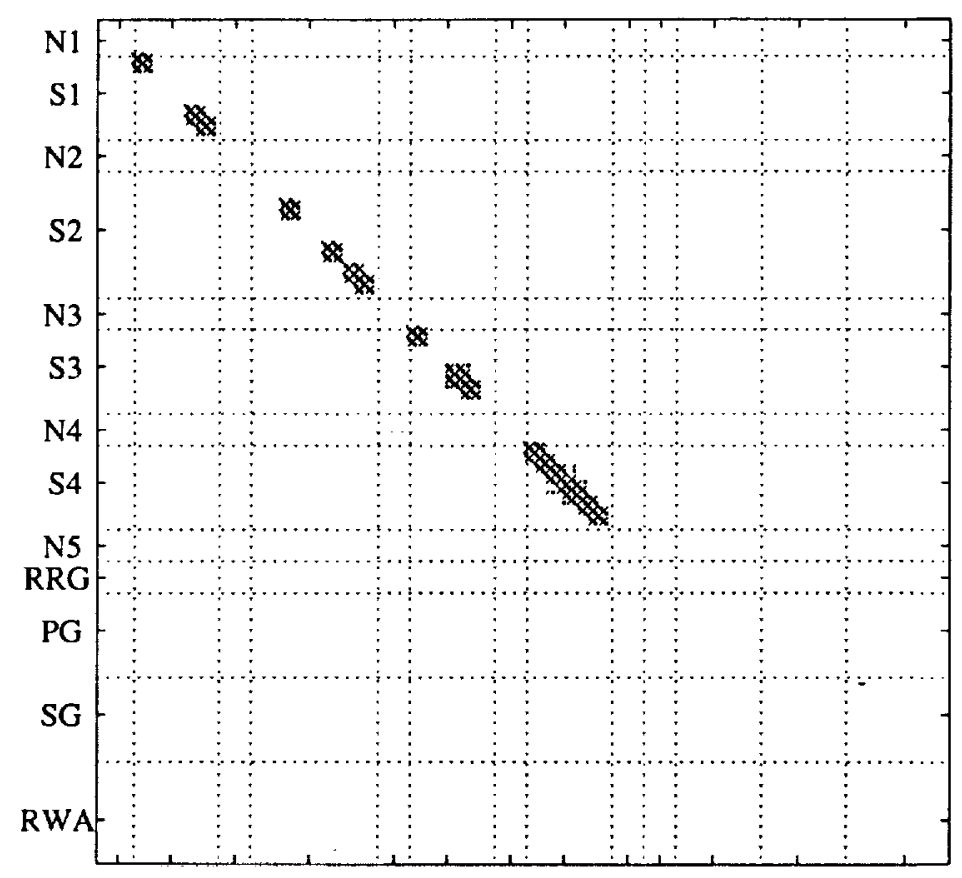

(a) Localized stiffness matrix mean error uncertainties.

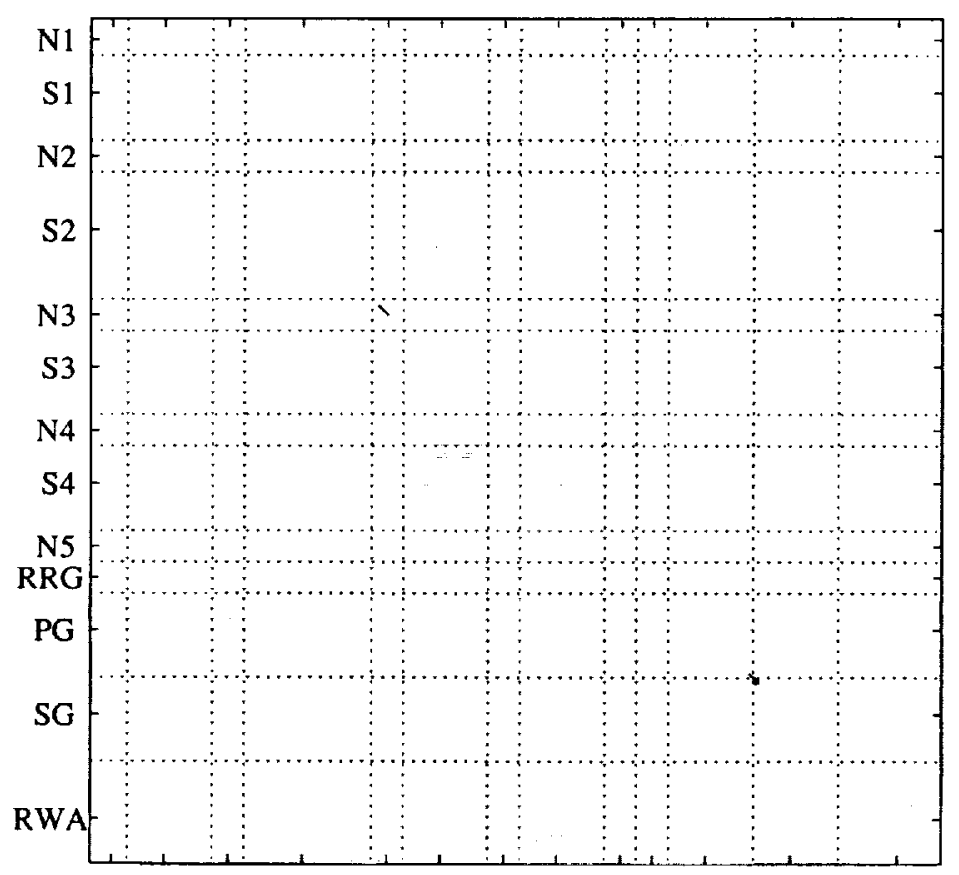

(b) Localized mass matrix mean error uncertainties.

Figure 5.8: MACE 0-g Configuration I localized mean error uncertainties. 


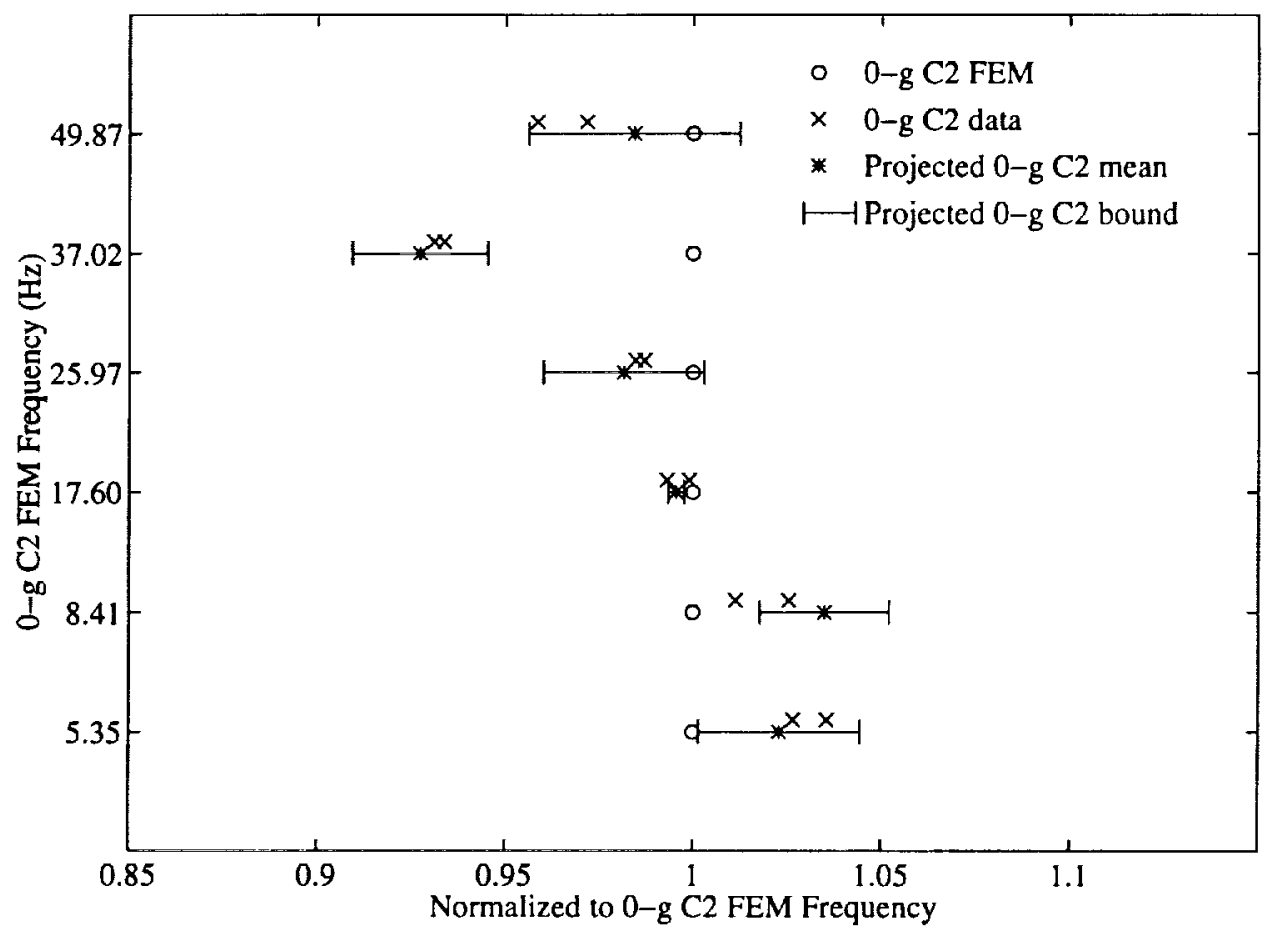

(a) XY-axes projection.

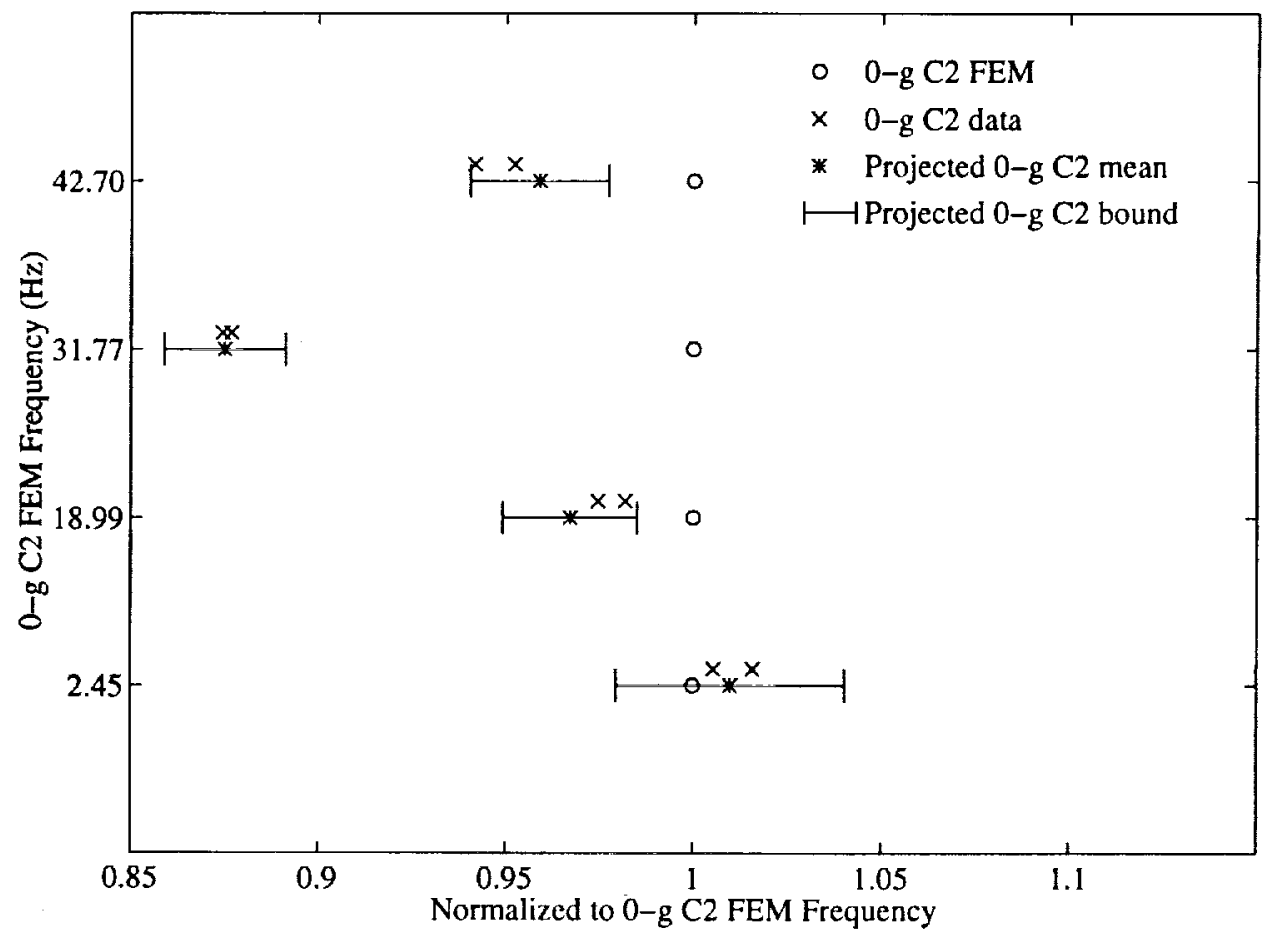

(b) Z-axis projection.

Figure 5.9: MACE 0-g Configuration I to Configuration II (C2) projection using the sensitivity method. 


\begin{tabular}{|c|c|c|}
\hline 0-g C1 Frequency (Hz) & FEM 0-g C1 to C2 (\%) & Data 0-g C1 to C2 (\%) \\
\hline \hline 2.26 & -4.6 & -8.0 \\
\hline 3.73 & -53.0 & -43.4 \\
\hline 9.46 & 13.6 & 11.1 \\
\hline 16.11 & -18.3 & -17.8 \\
\hline 17.84 & 0.5 & 1.3 \\
\hline 25.03 & -4.5 & -3.7 \\
\hline 39.56 & 26.9 & 19.7 \\
\hline 49.18 & 8.8 & 13.1 \\
\hline 49.67 & 28.6 & 25.4 \\
\hline 56.97 & 0.5 & 12.4 \\
\hline
\end{tabular}

Table 5.4: MACE 0-g Configuration I to Configuration II changes

Figure 5.10 shows two transfer functions of the Configuration II finite element model, the Configuration II finite element model shifted by the mean errors of the projected uncertainty model, and the corresponding 0-g Configuration II transfer function data. In both cases, the predicted transfer functions look more accurate, especially at higher frequencies.

The final aspect of this example investigated was the examination of the localized errors for Configuration II of MACE. Figure 5.11 shows the localized mass and stiffness errors for Configuration II. Again the same general trends exist: little mass error and errors in the active strut and ends of the passive struts. Although it is difficult to compare the 0-g Configuration I and Configuration II localized errors, qualitatively the errors localized tended to be quite similar. 


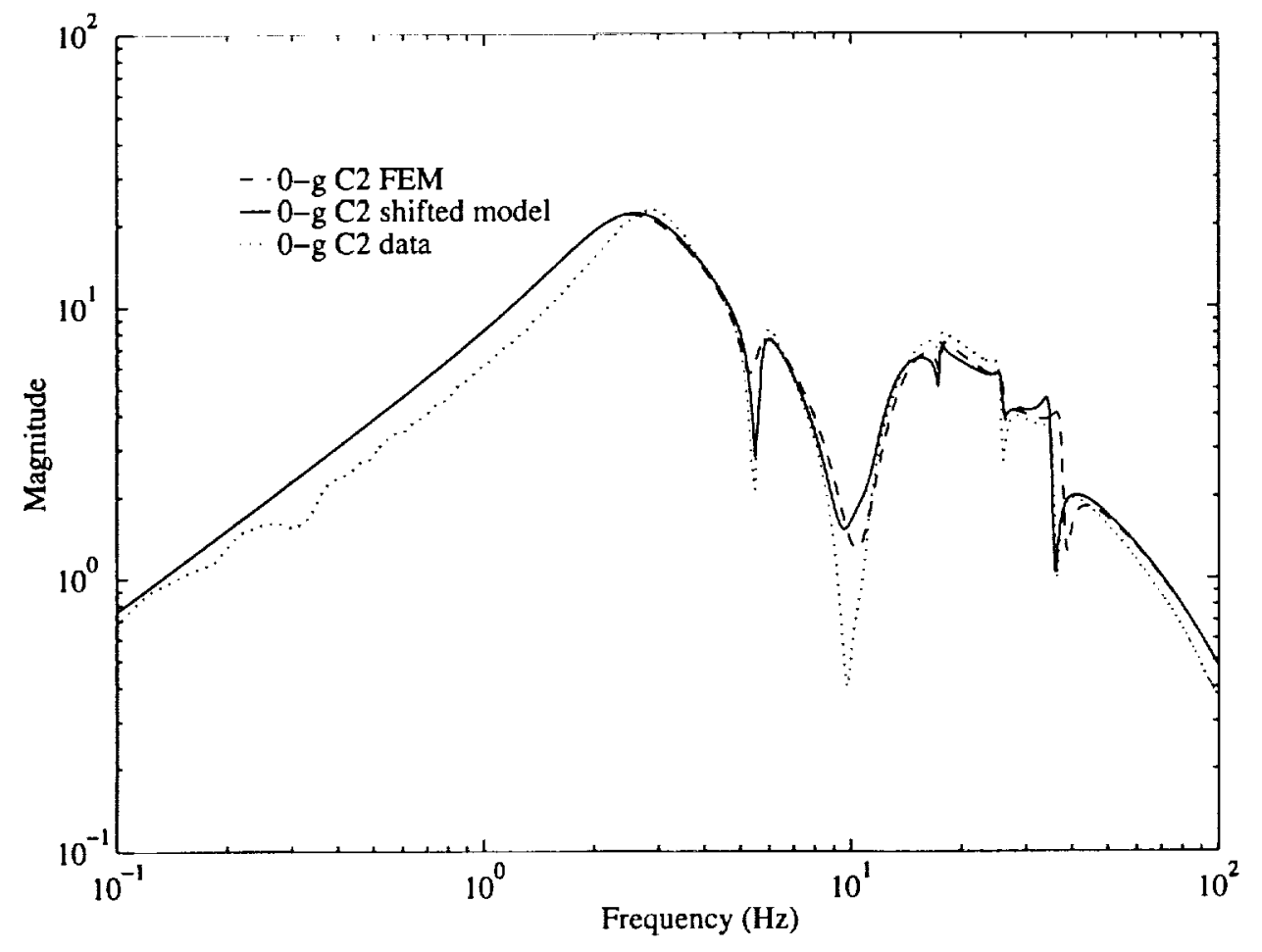

(a) Primary X Gimbal to Primary X Rate Gyro.

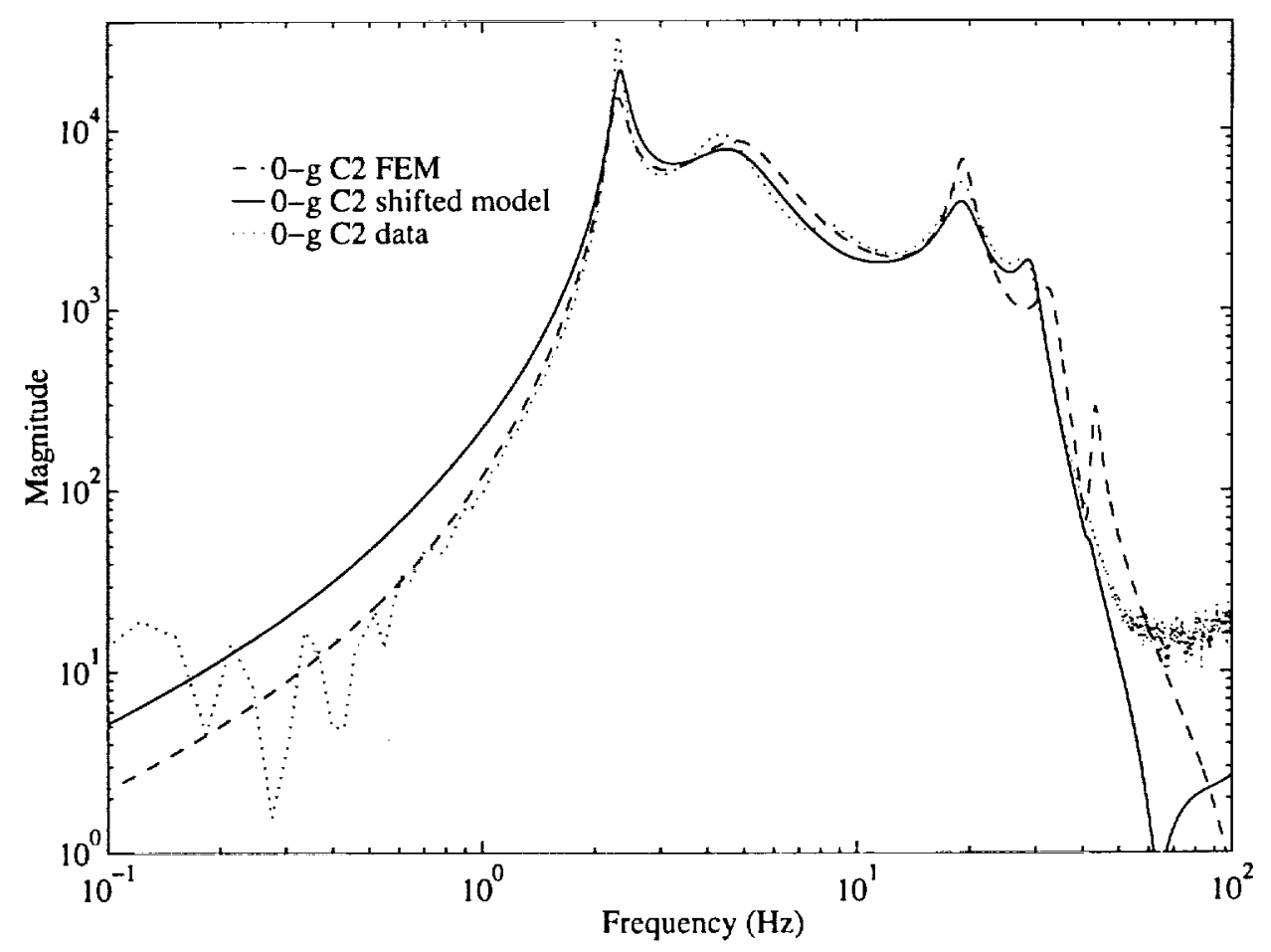

(b) Primary Z Gimbal to Strut 3 Z Strain Gauge.

Figure 5.10: MACE 0-g Configuration II (C2) FEM, 0-g C2 FEM shifted by projected mean errors, and one set of data. 


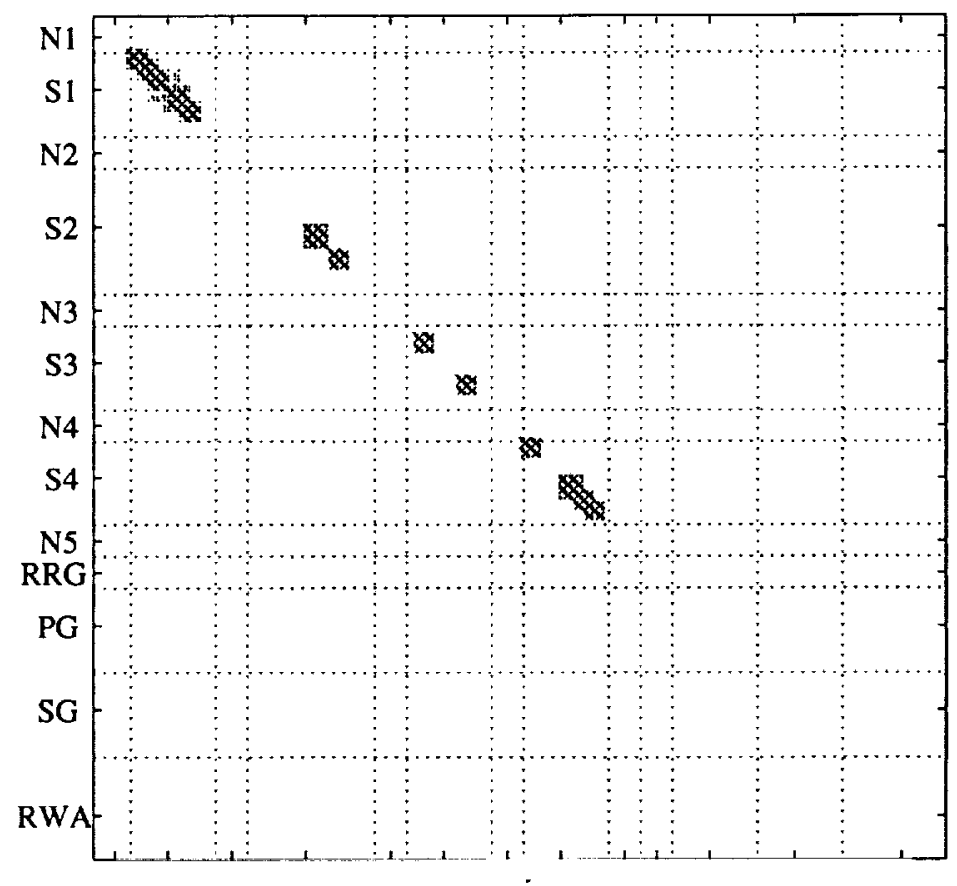

(a) Localized stiffness matrix mean error uncertainties.

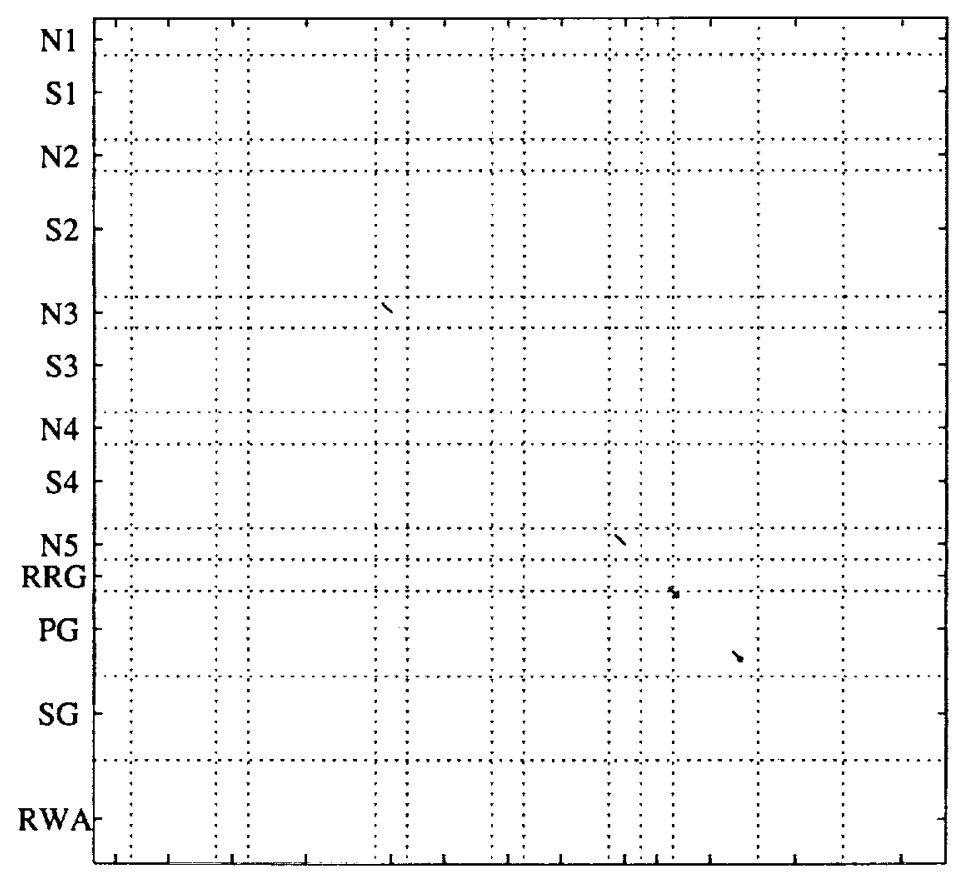

(b) Localized mass matrix mean error uncertainties.

Figure 5.11: MACE 0-g Configuration II localized uncertainties. 


\section{Chapter 6}

\section{Controller Synthesis and Analysis}

This chapter describes the use of an uncertainty model as a tool for control synthesis and analysis. The uncertainty model, in the form of mean errors and bounds on critical modal parameters of a finite element model, can be from either the nominal or modified systems. This chapter demonstrates the usefulness of an uncertainty model in control design on flexible systems. First, the general control design problem for uncertain systems is presented. An overview of controller synthesis and analysis methods which benefit from the use of an uncertainty model is given. In addition, the controller synthesis and analysis techniques used for the MACE flight are detailed showing an application of the uncertainty model for control design in an experimental setting. These methods make use of the finite element model, uncertainty model, and a real- $\mu$ analysis tool. The use of these methods is demonstrated experimentally using $0-\mathrm{g}$ controllers for MACE that were designed using the predicted 0-g uncertainty model. In addition, the conservatism of the uncertainty model is examined for each of the controllers presented. 


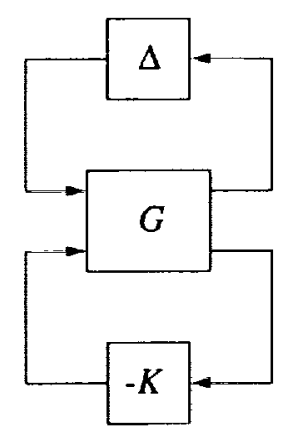

Figure 6.1: Interconnection of plant $G$, controller $K$, and uncertain block $\Delta$.

\subsection{Control of Uncertain Systems}

The design of controllers of uncertain structural systems is one of the most challenging problems in the field of controlled structures. Stringent performance requirements necessitate the use of high authority controllers. The success of these controllers, however, is directly linked to the accuracy of the modeling of the system and uncertainties, as well as the ability of the robust control design technique to handle the uncertainties.

Uncertainties in a structural system which are important to control design are usually caused by both unmodeled and mismodeled dynamics. Unmodeled uncertainties are usually at higher frequencies, and are treated using an unstructured block as shown in Figure 6.1, where $G$ is the plant dynamics, and $\Delta$ is an unstructured uncertainty block. Although this type of uncertainty is important, it does not limit the achievable performance in the problem described in this work. It is therefore not examined here.

Mismodeled dynamics are the primary cause of the parametric uncertainty similar to that in the uncertainty model described in this work, i.e. mean errors and bounds on the modal parameters. Because of the direct form of the uncertainty, the $\Delta$ block in Figure 6.1 can be structured to fit the type of uncertainty for which the system is being designed/examined. This is the type of uncertainty for control design which is examined here. 


\subsubsection{Controller Synthesis}

Techniques used to design controllers which are robust to parametric uncertainty can essentially be divided into six distinct categories: norm-based, $\mu$, multiple model, stochastic, de-sensitizing and classical. A brief overview of these methods is given here.

Norm-based methods are those methods in the $\mathcal{H}_{2}$ and $\mathcal{H}_{\infty}$ framework which address the problem of parametric uncertainty. Guarantees on robust stability using an unstructured, complex block can be made using an $\mathcal{H}_{\infty}$ control design technique [67]. In the $\mathcal{H}_{2}$ framework, robustness is added by coupling an $\mathcal{H}_{\infty}$ uncertainty test with an $\mathcal{H}_{2}$ performance metric [68].

To reduce the conservatism inherent in a single complex block, the $\mu$-synthesis technique was developed [69]. However, these approaches are known to be conservative for systems with constant real parameter uncertainties, i.e. the uncertainty model in this work. Therefore, the real $\mu$ and mixed $\mu$ techniques were developed to handle real parameter uncertainties [69-71]. In a series of papers, How et al. showed how the upper bounds for mixed $\mu$ are a generalization of the absolute stability criterion, where linear and nonlinear real parameter uncertainties are analyzed [72]. In addition, Popov controller synthesis was developed [73-75], where an $\mathcal{H}_{2}$ performance metric can now be introduced which gives a much tighter bound on the real parameter uncertainty.

Multiple model techniques have been used recently to gain robustness to parametric uncertainty by designing controllers around several models of an uncertain system $[76,77]$. Although there are no guarantees, less conservative closed loop results with a smaller amount of computation are usually achieved. Stochastic techniques such as Maximum Entropy [78,79] have been developed which introduce a multiplicative white noise model to capture the parameter uncertainty of the system. De-sensitizing techniques usually take an already proven unrobust method such as LQG and de-sensitize it to variations in parameters. For example, Blelloch and Mingori [80] modify the state and noise weighting matrices in the LQG compensator 
design to account for structured parametric uncertainty, thus reducing optimality. Sesak and Likins [81] add sensitivity states which penalize the variation of the performance objective with respect to parameter variations. These states can be eliminated from the model using a singular perturbation technique.

Although fairly ad hoc, classical techniques have been used for many years in designing controllers, many of which had plants that were uncertain. More recently, ties between classical and robust techniques have been made to gain insights into how the robust methods work, and to develop better classical methods [82-85].

\subsubsection{Controller Analysis}

The objective of controller analysis is to examine stability of controllers around an uncertain plant. Because of its close relationship to the synthesis of controllers, many of the controller analysis techniques are directly linked to the controller synthesis techniques. This is especially true of those design techniques which obtain stability guarantees. The analysis techniques that are discussed here examine the stability of any controller given a model of the plant and uncertainty.

The most simple analysis techniques are the classical graphical Nyquist and Nichols plots which have been adapted to contain uncertainty [86]. For flexible systems, however, these techniques primarily address the higher frequency unmodeled dynamics issue. Polynominal techniques such as Routh-Hurwitz [86] and Kharitinov's Theorem [87] examine the characteristic polynomial of the system to test stability. For complicated plants, however, these results are very conservative.

Another type of analysis technique that was developed during the MACE program is using the open loop data to predict the closed loop stability and performance [88]. The controller is wrapped around the open loop data to examine both the model and data, using the Nichols and sensitivity plots. Although there are no guarantees, this technique works quite well for systems which are primarily linear and repeatable. If these conditions do not exist, the ability of the method to predict closed loop stability and performance degrades.

The two methods that seem to work the best for the general case are a $\mu$ test 
and Monte Carlo simulation. The $\mu$ test [89] can be used to test for stability in the presence of complex or real uncertainties. The same difficulties exist, however, such as the real $\mu$ test not being continuous [71]. The Monte Carlo simulation can be performed where each of the parameters in the uncertainty model are allowed to vary, and a large number of cases are run to test for stability. This has the obvious limitations of large computational effort and no guarantees. A simplification of the Monte Carlo simulation is analyzing the controller on only a few systems, as in the multiple model controller synthesis. Although there are no guarantees, this drastically reduces the required computational effort.

\subsubsection{Utilization of the Uncertainty Model}

In addition to the use of controller synthesis and analysis techniques, the use of the uncertainty model in this process needs to be addressed. The uncertainty model is in the form of mean errors and bounds on the frequencies, modes shapes, and even damping (from 1-g). The next question is how to use all of this information in the control design procedure.

Figure 6.2 shows four options of using the uncertainty model. Given the nominal parameter set $\theta_{0}$, the uncertainty model consists of mean errors $\overline{\Delta \theta}$ and bounds $\widehat{\Delta \theta}$ on these parameters. The first option is the least conservative: shift the finite element model by the mean errors to create a control design model for synthesis; analyze using the predicted bounds as symmetric parameter variations. This case is termed "shiftedsymmetric". The next two cases are intermediate, such that asymmetric parameter variations are used, and the control synthesis model is either the original finite element model shifted by the mean errors, or just the finite element model. These two cases are termed "shifted-asymmetric" and "unshifted-asymmetric" respectively. The final case is the most conservative one in which the finite element model itself is used for controller synthesis, and the parameter variations are symmetric and include both the predicted mean errors and bounds. This is shown in Figure 6.2 as the "shiftedasymmetric" case. The choice of the application of the uncertainty model is made based on the confidence of the prediction of the uncertainty model. 


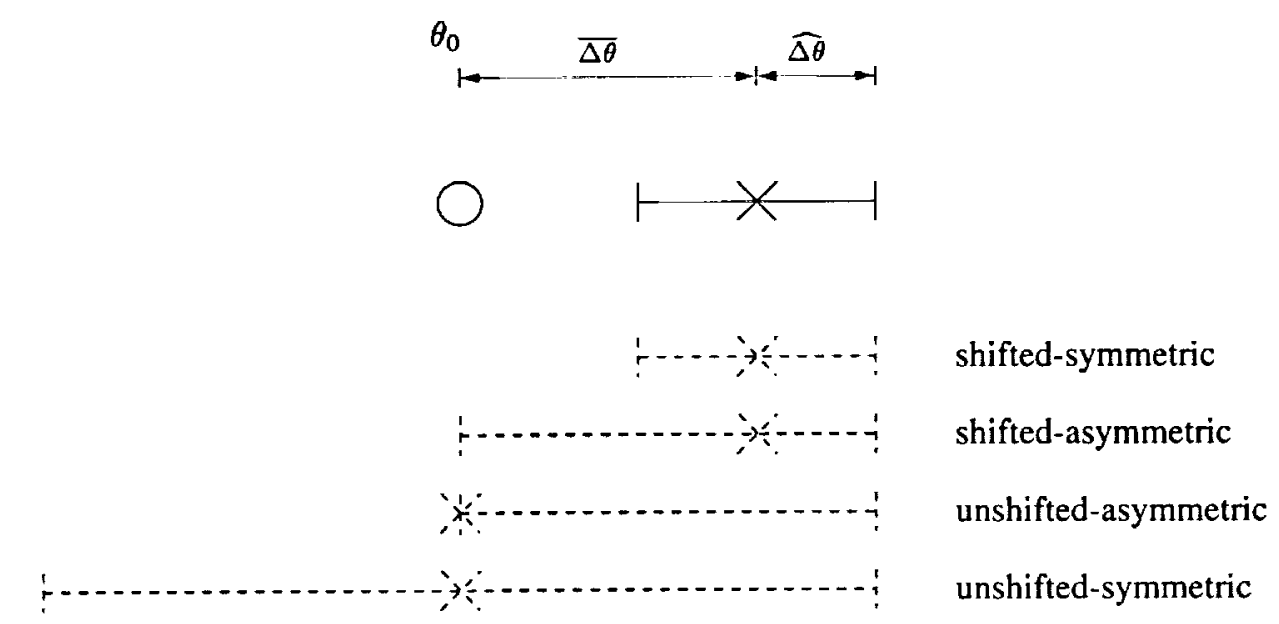

Figure 6.2: Types of controller synthesis and analysis using an uncertainty model

\subsection{MACE 0-g Results}

Controllers for the MACE flight were designed in terms of "families". Each family consisted of six to eight controllers, each designed the same type of robust control technique. As each controller was designed, the next controller built on the knowledge of the previous design, and increased the control authority. Therefore, the first controller in a family was usually lower authority, while the last controller was designed to really push the limits of the technique, finite element model, and uncertainty model.

\subsubsection{Controller Synthesis}

Controller synthesis for the MACE flight took the form of two approaches. One was the design of classically rationalized controllers as described in Ref. 85. While these controllers worked quite well on-orbit, they did not perform as well as the second option [44]. The second option was a blending of techniques that were examined in Refs. 90, 88. First, a lower authority LQG controller [91] is designed to understand tradeoff between the regulator and estimator, and areas where robustness may be needed. Next, a de-sensitizing method called Sensitivity Weighted LQG (SWLQG) $[92,90]$ is used to add robustness to the LQG controller by making an 
adjustment to the state costs of the regulator and estimator problems. Finally, the Multiple Model technique $[76,77]$ is used as the final design to really push the limits of controller authority. The SWLQG controller from the previous design is used as an initial guess in the optimization. This blending of techniques has worked quite well in designing high authority robust controllers in 1-g and 0-g. Although other robust control design methods could handle the uncertainty model presented, the multiple model technique, even though there are no closed loop guarantees, achieved the best closed loop results for the computational effort needed. This factor of a lower computational effort became a significant factor for redesign of controllers while MACE was on-orbit.

\subsubsection{Controller Analysis}

Controller analysis for the MACE flight was again in two forms. The first was the use of open data to predict the closed loop stability and performance by examining the Nichols and sensitivity plots [88]. This method, however, could only be used during the flight when open loop data was available (after it had been downlinked.) Prior to flight, a mixed- $\mu$ analysis using the finite element model and the predicted uncertainty model was used $[48,93]$. This method is detailed here.

For the MACE flight, there were no uncertainties developed for the mode shapes. Therefore, only uncertain frequencies and damping ratios were considered in the development of the mixed- $\mu$ analysis tool.

For a system represented in state space as

$$
\begin{aligned}
& \dot{x}=A x+B u \\
& y=C x+D u,
\end{aligned}
$$

the frequency and damping ratio variations are treated as uncertainties in the $A$ matrix of the system dynamics. Each mode of the system can be represented with a $2 \times 2$ matrix, $A_{2 \times 2}$, of the following form

$$
A_{2 \times 2}=\left[\begin{array}{cc}
0 & 1 \\
-\omega_{0}^{2}\left(1+2 \alpha_{\omega} \delta_{\omega}\right) & -2 \zeta_{0} \omega_{0}\left(1+\alpha_{\zeta} \delta_{\zeta}\right)
\end{array}\right]
$$


where $\delta_{\omega}, \delta_{\zeta} \in[-1,1]$ are the uncertain real parameters and $\alpha_{\omega}$ and $\alpha_{\zeta}$ are real scaling factors that represent the percent uncertainty in the frequency and damping ratio, respectively. Note that for systems with lightly damped modes, $\zeta \ll 1$ and for $\alpha_{\omega} \ll 1$, treating uncertainty in the square of the frequency is a good approximation to half the uncertainty in the frequency alone. This matrix can then be represented using the internal feedback loop representation [94]

$$
\begin{aligned}
\dot{x} & =A_{0} x+B_{0} p \\
q & =C_{0} x \\
p & =\Delta q .
\end{aligned}
$$

where

$$
\begin{gathered}
A_{0}=\left[\begin{array}{cc}
0 & 1 \\
-\omega_{0}^{2} & -2 \zeta_{0} \omega_{0}
\end{array}\right], B_{0}=\left[\begin{array}{ll}
B_{0 \omega} & B_{0 \zeta}
\end{array}\right], \\
C_{0}=\left[\begin{array}{c}
C_{0 \omega} \\
C_{0 \zeta}
\end{array}\right], \Delta=\left[\begin{array}{cc}
\delta_{\omega} & 0 \\
0 & \delta_{\zeta}
\end{array}\right], B_{0 \omega}=\left[\begin{array}{c}
0 \\
-2 \alpha_{\omega} \omega_{0}
\end{array}\right], \\
B_{0 \zeta}=\left[\begin{array}{c}
0 \\
-\alpha_{\zeta} \omega_{0}
\end{array}\right], C_{0 \omega}=\left[\begin{array}{ll}
\omega_{0} & 0
\end{array}\right], C_{0 \zeta}=\left[\begin{array}{ll}
0 & 2 \zeta_{0}
\end{array}\right] .
\end{gathered}
$$

With the uncertainty loops closed,

$$
A_{2 \times 2}=A_{0}+B_{0} \Delta C_{0}
$$

which is exactly as in Equation 6.2.

An analysis tool was sought which investigated the stability of the system for all possible perturbations included in the uncertainty model. This type of uncertainty lends itself to a real $-\mu$ analysis of the system. However, because real $\mu$ is not continuous in the problem data [71], this tool cannot be used for the practical system such as MACE. If a complex block could be added to the uncertainty, however, the problem would be continuous, and therefore increase its usefulness for the MACE system.

Drawing upon the evaluation of controllers using data, where the sensitivity singular values are plotted using the model and data and discrepancies are used as a guide 
in designing robust controllers, a weighted sensitivity block is added. The sensitivity transfer function $(I+G(j \omega) K(j \omega))^{-1}$, provides a measure of how close to singularity, i.e. instability, the closed loop system is. If the complex block is defined as the weighted maximum singular value of the sensitivity, or $1 /\left(\gamma \bar{\sigma}(I+G(j \omega) K(j \omega))^{-1}\right)$, the weighted complex block implies that no allowable parameter shift can cause the sensitivity to peak more than $\gamma$ above the nominal sensitivity.

Large deviations between the singular values as calculated using the model and using measured data indicate where modeling errors may cause stability problems. As such, the weighting function used in the sensitivity loop is the inverse of the nominal maximum singular value of the sensitivity.

The final aspect of the mixed- $\mu$ analysis tool is the scale factor for the weighting function. Obvious limits for this scaling factor are 1 and $\infty$. Using a scaling factor of 1 tests whether or not the maximum singular value of the sensitivity transfer function increases for any allowable perturbations. This is clearly too stringent a test. Using a scaling factor of $\infty$ ignores the sensitivity loop altogether and tests only stability. Through experimentation and comparing the sensitivity transfer function calculated using both the model and measured data for compensators that stabilized and destabilized the system, it was found that a scaling factor of 2 achieved good prediction results. However, if the nominal sensitivity transfer function is large ( $>3$ or 4) over a particular frequency range, then a smaller scaling factor should be used.

Figure 6.3 shows the interconnection structure of uncertainty inputs and outputs of this mixed- $\mu$ test. The weighting function, $W$, is the scaled inverse of the maximum singular value of the nominal sensitivity transfer function. Stability of this system, achieved if $\mu<1$, implies that the deviation in the maximum singular value of the sensitivity transfer function will be no more than a factor of 2 for any feasible perturbation.

\subsubsection{Utilization of the Uncertainty Model}

For the MACE flight, the predicted 0-g uncertainty model was developed using the matrix method presented in the previous chapter. The frequencies are shown in Fig- 


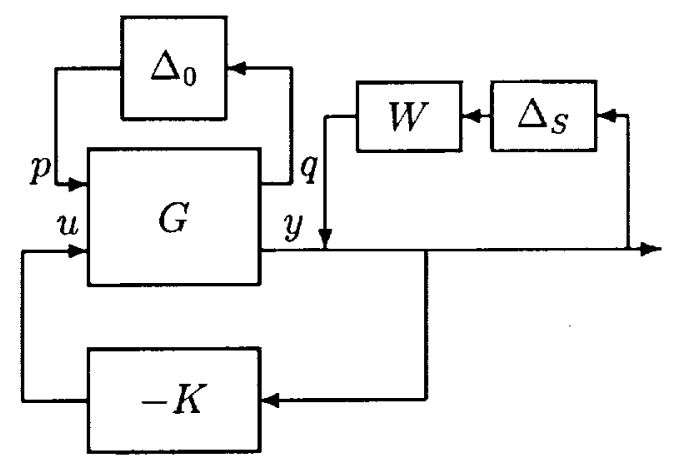

Figure 6.3: Interconnection of the open loop system $(G)$, compensator $(K)$, real parameter uncertainty $\left(\Delta_{0}\right)$, and weighting on the Sensitivity $(W)$, and the Sensitivity delta block $\left(\Delta_{S}\right)$.

ure 5.2. Although the 0-g data was not available, it was known that the matrix method did not provide a physically based solution. Therefore, confidence in the prediction of the $0-\mathrm{g}$ uncertainty model was lacking. Because of this, most of the pre-programmed controllers for the MACE flight were designed using the most conservative utilization of the uncertainty model, or the "symmetric-unshifted" case in Figure 6.2.

Figure 6.4 shows an example of how the mixed- $\mu$ analysis tool and uncertainty model were used in controller analysis and synthesis. The uncertainty model used is the most conservative, unshifted-symmetric case. The parameters in the uncertainty model are the frequencies an damping ratios only. Because of the difficulties in predicting the 0-g damping, $50 \%$ variations were used in the uncertainty model.

Figure 6.4(a) is a plot of $\mu$ for an LQG controller designed for a sample system. Notice the high values of $\mu$ in the $3.3,10$, and $17 \mathrm{~Hz}$ ranges. This indicates areas that need to be addressed with robust control in the synthesis procedure. This is accomplished in an iterative fashion. Figure 6.4(b) shows a plot of $\mu$ for a SWLQG controller on the same topology, at the same controller authority, and robustified in each of the problem areas. Note the $3.3,10$, and $17 \mathrm{~Hz}$ ranges are all under 1 , indicating the design is robust to the given changes in the uncertainty model. 


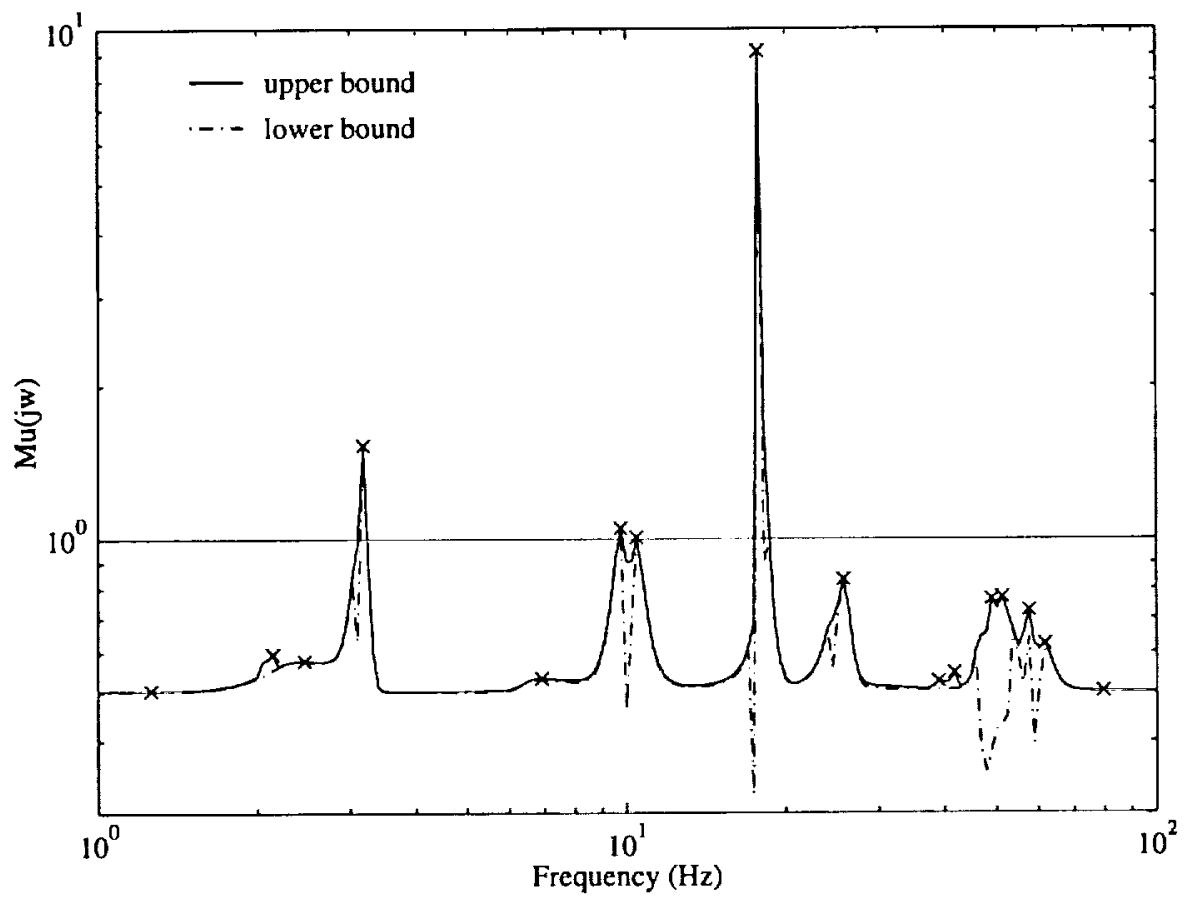

(a) Plot of mixed- $\mu$ for an unrobust LQG controller.

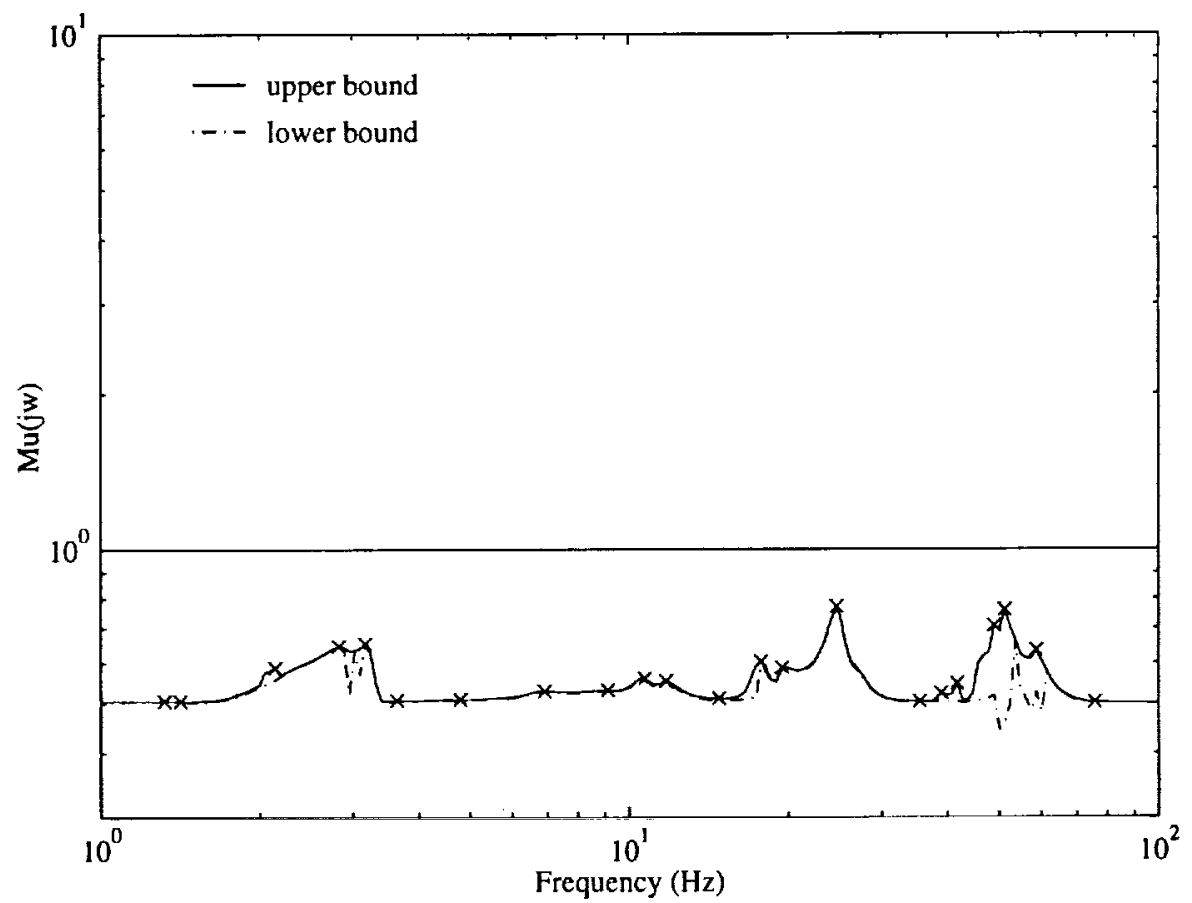

(b) Plot of mixed- $\mu$ for a robust SWLQG controller.

Figure 6.4: Example of the usage of the mixed- $\mu$ analysis tool, and the uncertainty model. 


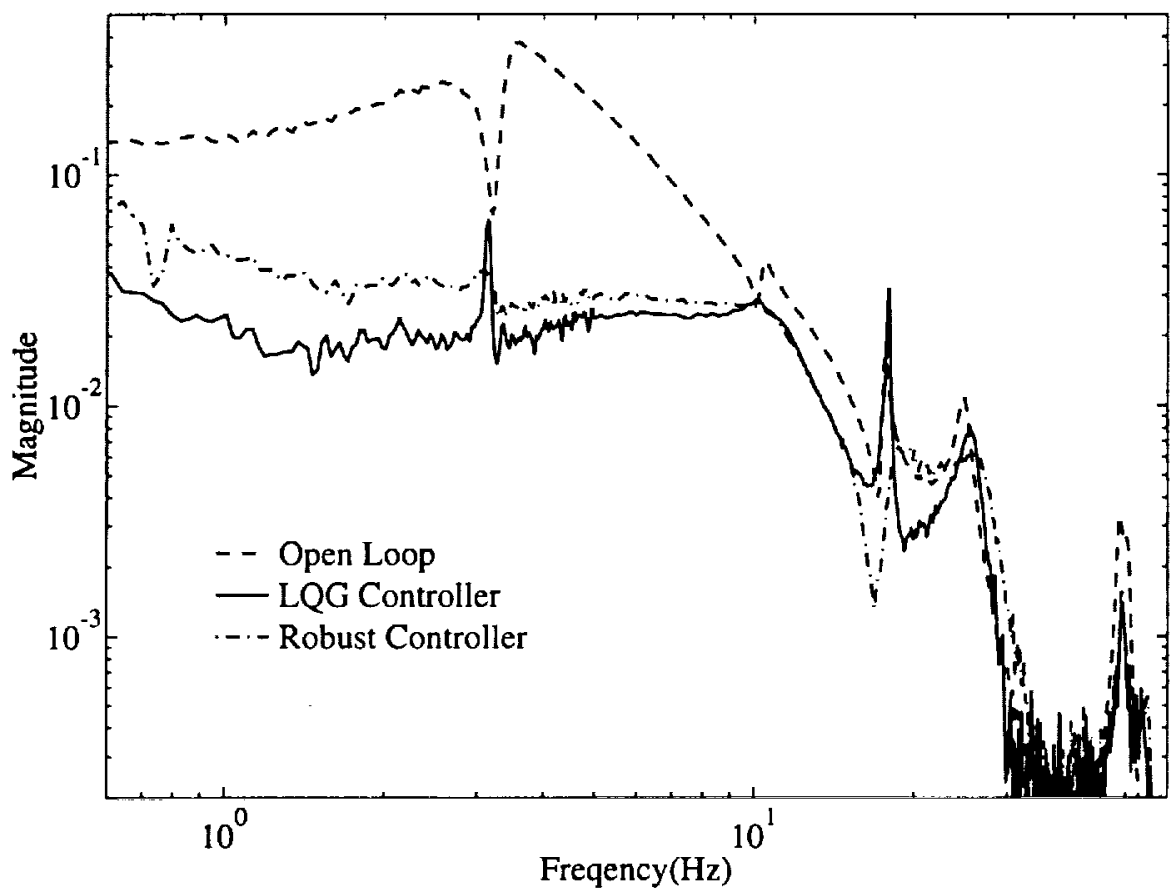

Figure 6.5: MACE 0-g closed loop results using for LQG and SWLQG controllers.

\subsubsection{Closed Loop Results}

The first set of closed loop results presented corresponds to the LQG and SWLQG controllers evaluated in Figure 6.4. The topology is an X axis design, as shown in Figure 5.1(a): the disturbance enters the $X$ axis secondary gimbal; the performance is the integrated $\mathrm{X}$ axis rate gyro in the primary payload; the inputs are the $\mathrm{X}$ and $\mathrm{Y}$ axes reaction wheels; and the outputs are the $\mathrm{X}$ and $\mathrm{Y}$ axes rate gyros at the center node, and the $\mathrm{X}$ axis rate gyro. Figure 6.5 shows the closed loop results for these two controllers. Although the LQG controller is not unstable, it is quite sensitive in each of the predicted areas: $3.3,10$, and $17 \mathrm{~Hz}$. The SWLQG controller is stable and quite robust, as predicted.

The next topology is the benchmark control topology. This topology is a twodisturbance, five performance, five input, and five output system. Examining Configuration I of MACE in Figure 5.1(a), two disturbances enter into the secondary gimbal in the $\mathrm{X}$ and $\mathrm{Z}$ axes; the five performances are each integrated rate gyros in the $\mathrm{X}$ 
and $Z$ axes of the primary payload, and $X, Y$, and $Z$ axes of the rate gyros at the center node; the five inputs include the three reactions wheels at the center node, and $\mathrm{X}$ and $\mathrm{Z}$ axes of the primary gimbal; and the five outputs are the five rate gyros at the center node and in the primary payload.

Closed loop controllers were designed and implemented on MACE in 0-g to examine the tradeoffs of using the uncertainty model. Two controllers are examined for this topology. The first was designed and analyzed using the most conservative, unshiftedsymmetric case in Figure 6.2. The second controller was designed and analyzed using the most unconservative case of symmetric-shifted in Figure 6.2. Multiple-model control design was used for both cases, although different authorities are presented primarily because the less conservative case allowed for higher authority.

Figure 6.6 shows a plot of the combined closed loop metric as a function of frequency. The performance metric weights the five individual performances with 1 for each of the gimbal integrated rate gyros, and 0.1 for each of the center node rate gyros, as this is a pointing experiment. The "Open Loop" system is self-explanatory; the "Servos-Only" system is using the first layer of control which is the four encodergimbal servo systems; "Unshifted-Symmetric FEM CST" is the best pre-programmed high-authority controller using the unshifted finite element model and symmetric parameter variations covering both the predicted mean errors and bounds of the uncertainty model; "Shifted-Symmetric CST" is the best pre-programmed high-authority controller using the shifted finite element model and symmetric parameter variations derived using only the predicted bounds of the uncertainty model. Notice that the less conservative shifted-symmetric controller performs $2.3 \mathrm{~dB}(7 \%)$ better than the unshifted-symmetric controller. This shows the benefits of using the the less conservative shifted-symmetric approach for controller synthesis and analysis.

There are a few drawbacks, however, because of the inaccuracies of the matrix method based uncertainty model. The first can be seen in the $17 \mathrm{~Hz}$ range as an increase in sensitivity of the closed loop system. The $17 \mathrm{~Hz}$ mode was one of the frequency errors that the matrix method based prediction was incorrect (Figure 5.2). In addition, closed loop systems for both controllers are sensitive at $50 \mathrm{~Hz}$, primarily 


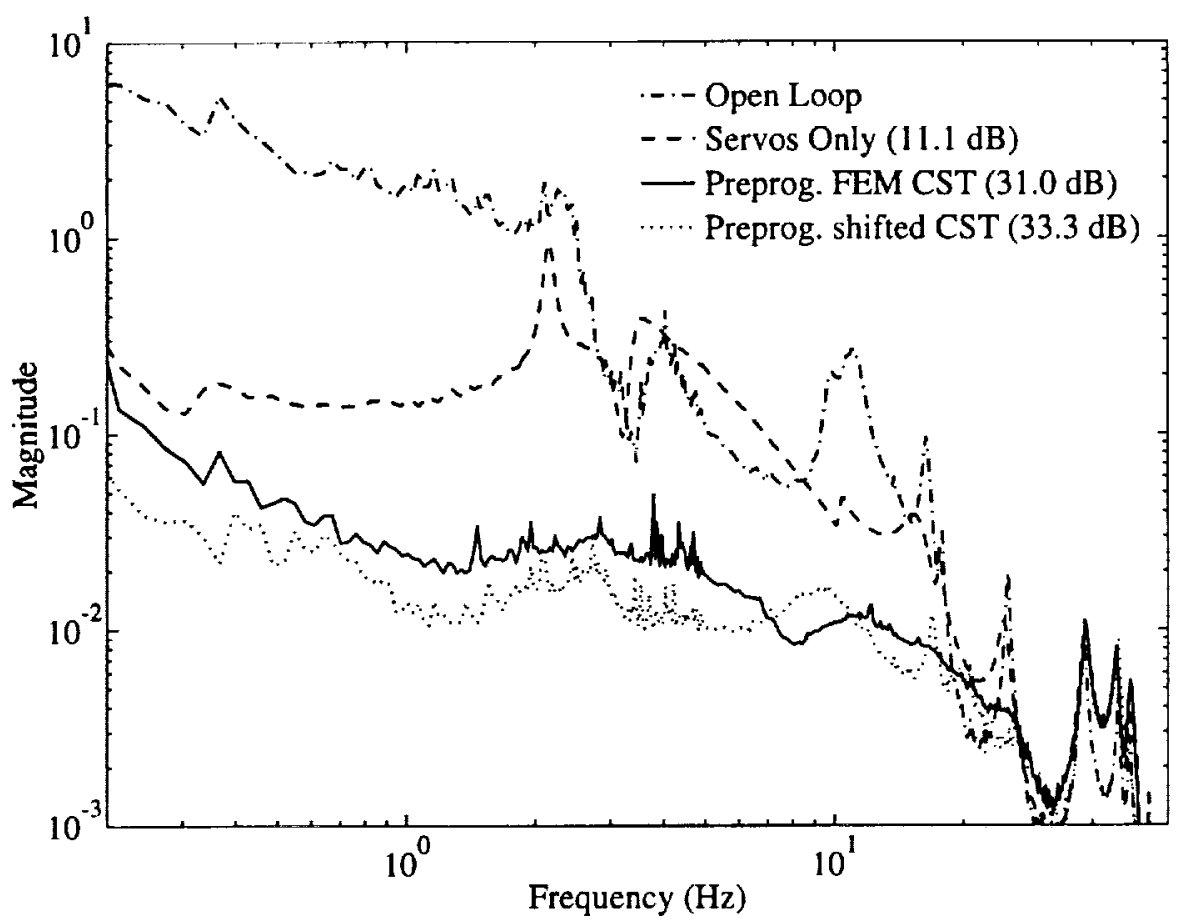

Figure 6.6: MACE 0-g closed loop results using unshifted model/symmetric bounds (FEM) and shifted model/symmetric bounds (shifted).

because the uncertainty model did not predict the large errors in this frequency region. If the uncertainty model developed using the sensitivity method (Figure 5.6), which provides physical, as well as mathematical results, these problems may not have appeared.

It is debatable whether the increased performance was the result of good prediction of mean errors, or reduced conservatism in the bounds, especially since the basis of the uncertainty model used for the design of both controllers for flight was the nonphysical matrix method. In reality, it is probably a combination of both. It would be interesting to test new controllers designed using the improved sensitivity method, however, this is not a realistic scenario.

\subsubsection{Analysis of Uncertainty Model}

Although a redesign of controllers using the improved uncertainty model created using the sensitivity method was not a realistic option for this work, analyzing controllers 
that have already been implemented can be done. Figure 6.7 shows the mixed- $\mu$ plot of the "Pre-prog. FEM CST" controller in figure 6.6 for three different uncertainty models. Figure 6.7(a) shows the $\mu$ plot for the most conservative unshifted-symmetric case where the unshifted finite element model, and the symmetric parameter variations which cover both the predicted mean errors and bounds are used. Figure 6.7(b) shows the $\mu$ plot for the least conservative shifted-symmetric case where the finite element model is shifted by the predicted mean errors, and the symmetric parameter variations are just the predicted bounds of the uncertainty model. Figure 6.7(c) shows the $\mu$ plot for the actual $0-\mathrm{g}$ uncertainty model: the $0-\mathrm{g}$ finite element model shifted by the mean errors from the $0-\mathrm{g}$ data, and parameter variations consisting of the parameter bounds from the $0-\mathrm{g}$ data. The conservatism of the unshifted-symmetric case prevented the controller from achieving even greater performance. The shiftedsymmetric case, however, is quite similar to the actual 0-g data, implying the use of the uncertainty model generated from the sensitivity method would decrease the conservatism of the designs, and therefore increase closed loop performance. 


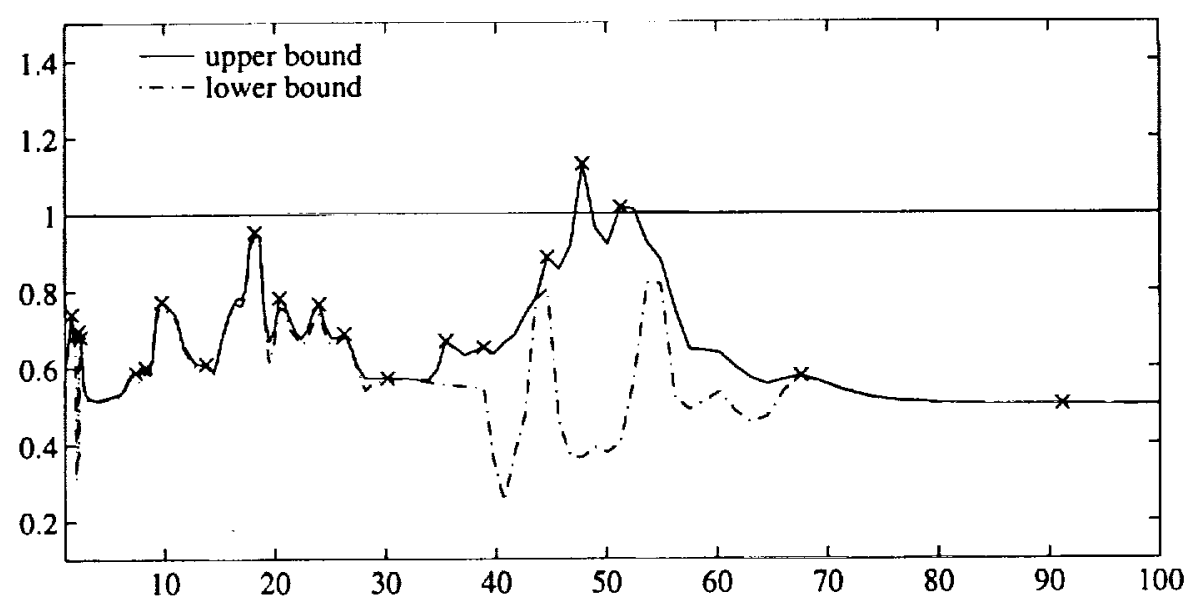

(a) Most conservative unshifted-symmetric case.

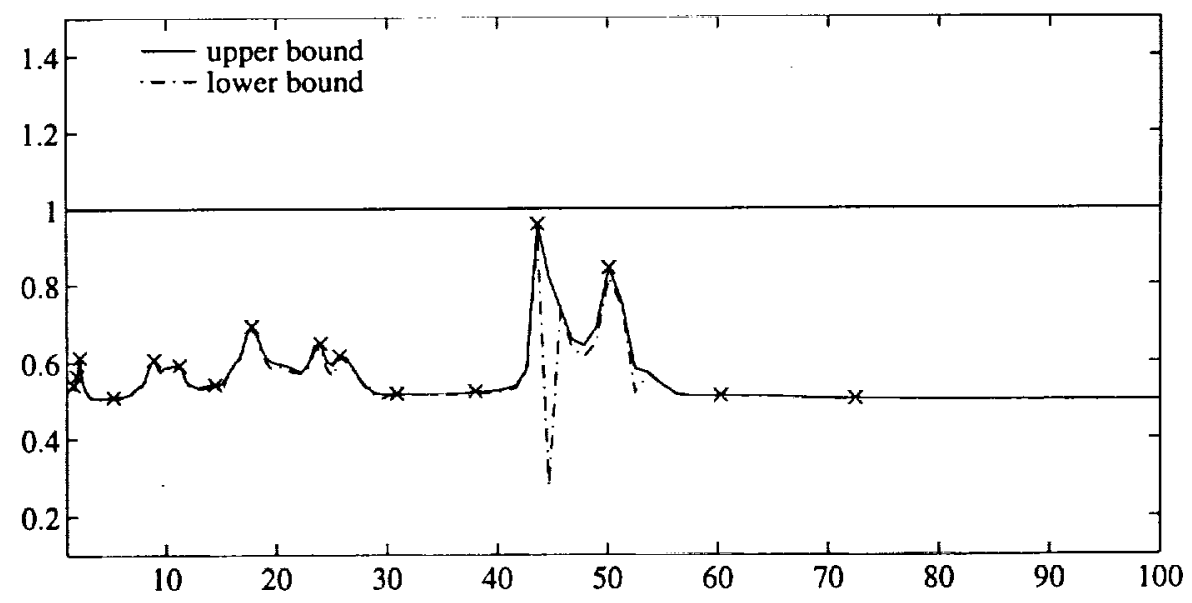

(b) Least conservative shifted-symmetric case.

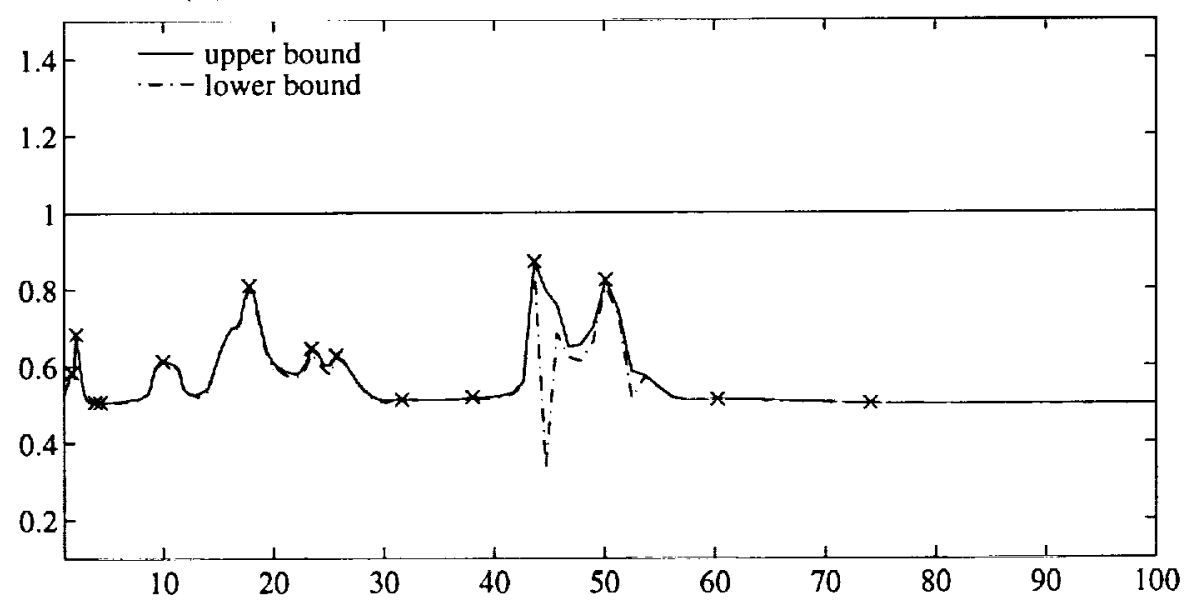

(c) 0-g measurement model and bounds.

Figure 6.7: Mixed- $\mu$ analysis of a $5 \times 5 \times \mathrm{XYZ}$ controller using three types of uncertainty models derived using the sensitivity method. 


\section{Chapter 7}

\section{Conclusions}

\subsection{Summary}

A set of methods to identify stochastic uncertainties between a finite element model and set of hardware in a nominal environment, and to predict stochastic uncertainty model a modified have been developed. The approach of this work is to first identify mean errors and bounds on critical parameters of the nominal system. These uncertainties are then localized to specific degrees of freedom in order to form mass and stiffness uncertainties. Once in this form, the uncertainties can be mapped and projected into the modified system to create an analogous uncertainty model.

Many uncertainties exist, mean and variance, between a set of hardware and structural model. In this work, a summary of these uncertainties is given. These are described by first the large groupings of linear and nonlinear uncertainties. Linear uncertainties are then sub-divided into the following groups: physical parameters such as the geometric and material properties; modeling uncertainties such as discretization and incorrect element physics; internal couplings such as joints and/or attachments and actuator and sensor dynamics; external dynamics such as aerodynamic, gravity, or closed loop coupling; testing uncertainties such as sensor and process noises and bias errors. The nonlinear uncertainties can be subdivided into two categories: weak and strong nonlinearities. Of these uncertainties, most are capturable using the methods developed in this work except for the modeling uncertainties and strong nonlinearities. 
These two types of uncertainties cannot be represented with either a mass and/or stiffness mean error or variance uncertainty.

Many of the uncertainties described can be found by thoroughly testing the structure. A testing summary is presented in this work which enables the observing of all of the uncertainties, both mean and variance. These tests involve varying testing parameters such as actuators, disassembly/reassembly, time between tests, disturbance levels, boundary conditions, and structural and non-structural changes.

The nominal or 1-g uncertainty model can be accurately described using mean errors and bounds on the frequencies and mode shapes of the nominal system. These are found by identifying the critical parameters of the system using an identification procedure that parameterizes the finite element model, actuator and sensor dynamics, and time delays separately. The Discrete Extended Kalman Filter (DEKF) was the method used to identify the parameters, although other methods could also have been used. The DEKF, in addition to estimating the parameters of the system, gives additional information in the form of an measurement error covariance on the parameters. Although this matrix is not the exact measurement error, it does give relative confidence factors in the estimation of the parameters. These confidence factors can then be used to find the weighted mean error and bound for each parameter of the uncertainty model. The DEKF appeared to work quite well as an identification/parameter estimation method in all sample problems and on experimental MACE data. Because only one identification/parameter estimation method was used, however, a more thorough examination of these types of methods is in order.

Two methods for localizing the uncertainties and projecting them into a modified, or 0-g environment have been developed. The first is called a matrix method, which manipulates the first order perturbation equations of the structural eigenvalue problem in order to develop the modified uncertainty model. The solution from this method, however, may give results that may be non-physical, in that pseudoinverses utilized during the derivation are on underdetermined systems. Therefore the mass and stiffness matrices may be non-physical, implying difficulties with the projection step. This was exemplified in the MACE examples, where the matrix method did not 
give consistent results.

A second method, termed the sensitivity method, builds on the first order perturbation by adding a specific form to the mass and stiffness uncertainties. This form is a combination of macroelements which represent possible physical uncertainties, and scale factors. Although a pseudoinverse is again used in the derivation of the sensitivity method, it is now on an overdetermined system, implying that the mass and stiffness uncertainties, as well as the projected modified or 0-g uncertainty model, are physically based, as well as mathematically based.

A practical algorithm using the sensitivity method is presented. This algorithm consists of first examining the relative numbers of measured parameters and possible mass and stiffness uncertainties. A layered approach is suggested, which first localizes uncertainties in groups of elements, then reduces the number of these groups; localizes uncertainties in elements, then reduces the number of these elements; and then localizes the uncertainties in sub-elements. In addition, if the mean uncertainties are large, an iterative procedure can be used where an eigenvalue problem is solved at each step, after the mean error localization.

The uncertainty localization and projection methods are demonstrated using both a sample problem, and the Middeck Active Control Experiment (MACE). The sample problem shows that the sensitivity method can be used to identify both mean error and variance uncertainties in the physical mass and stiffness matrices using partial information, i.e. a subset of measured modes and degrees of freedom. The matrix method, because it is non-physical, can only solve for the correct physical uncertainties by using all of the measured modes and degrees of freedom, which is not a realistic scenario. The sensitivity method is therefore the best method to use for localization and projection of uncertainties.

The MACE experiment gives experimental validation to the uncertainty model, localization, and projection methods developed in this work. Because of large number of data sets for MACE in $0^{-} \mathrm{g}$ as well as $1-\mathrm{g}$, the $1-\mathrm{g}$ to $0-\mathrm{g}$ projection could be completely examined. The sensitivity method performed quite well in predicting the mean errors and bounds, while the matrix method predictably did not. The localiza- 
tion step of the sensitivity method was quite difficult for this example, however. This is most likely caused by large errors in the modeling of gravity effects, and difficulties in localizing superposable uncertainties such as physical modeling errors and gravity modeling errors (using the small number of sensors given. It was therefore concluded that not only should mode shape uncertainties be used along with frequency uncertainties in the localization process, but a large number of sensors are needed for more difficult problems such as the $1-\mathrm{g}$ to $0-\mathrm{g}$ projection.

In another example using MACE, the uncertainty model of a second " $\mathrm{"} \mathrm{configu-}$ ration for MACE in 0-g was developed, based on the original straight configuration. The uncertainty localization and projection worked very well for this example, and did not have the difficulties of the $1-\mathrm{g}$ to $0-\mathrm{g}$ example. This was because there were no superposable gravity uncertainties to localize, therefore simplifying the problem immensely.

Finally, a chapter on utilizing an uncertainty model for control design and analysis is presented. A summary of methods for controller synthesis and analysis that would benefit from the use of an accurate uncertainty model is given. Four cases of utilizing the uncertainty model are recommended, depending on the confidence of the user in the uncertainty model. These are: shifted-symmetric, shifted-asymmetric, unshiftedasymmetric, and unshifted-symmetric, where the (un)shift operator implies that the critical parameters of the finite element model have been (un)shifted by the predicted mean errors of the uncertainty model, and the (a)symmetric operator implies the controller is designed and/or analyzed using (a)symmetric parameter variations created using the uncertainty model. Two 0-g controllers from the MACE flight are given which utilize the most conservative unshifted-symmetric case, and the least conservative shifted-symmetric case. Although both controllers performed well, the less conservative case performed slightly better, thus showing the usefulness of an accurate uncertainty model for control design. The closed loop MACE experiments showed that an accurate uncertainty model can be very helpful for controller synthesis and analysis. 


\subsection{Contributions}

1. Uncertainties or errors between a (finite element) model and set of hardware have been detailed in many sources, although they are usually incomplete. This work, however, gives the most complete description of structural modeling uncertainties of its kind, along with a set of tests to identify them. In addition, the uncertainties are uniquely identified as stochastic, rather than deterministic. Therefore, one of the more significant contributions of this work is the development of an uncertainty model, which consists of bounds, as well as mean errors, on critical parameters of a structural system.

2. Error localization and update methods for finite element models have been areas of on-going research for the past few decades. This work develops an additional method, called the matrix method, which localizes errors by manipulating the first order perturbation matrices of the structural eigenvalue problem. Although unique in its derivation, this method provides a non-physical solution. A second method, called the sensitivity method, builds upon the work of Collins et al. and Lallement et al. where a structure is given to the mass and stiffness errors. Although this is an already proven method, this work extends the previous work by providing a complete algorithm for the practical localization of errors. In addition, this work extends the sensitivity method in the significant contribution of including variance uncertainties, as well as mean errors. This provides the basis for modeling practical structures which are stochastic, rather than deterministic in nature.

3. Application of the uncertainty localization and projection methods to the problem of structural control in a different environment is a significant contribution. The idea of localizing mass and stiffness uncertainties in the nominal $1-\mathrm{g}$ environment, and projecting them into the modified $0-\mathrm{g}$ environment using the finite element models is quite unique. This allows the development of an uncertainty model in an environment that is distinct from the original, i.e. modified or $0-\mathrm{g}$. 
4. Because of the success of the MACE experiment, a large set of data in both 1-g and $0-\mathrm{g}$ has been collected which has allowed the experimental validation of the methods developed in this work. Two examples were used for this validation: the complex 1-g to 0-g transition, and the 0-g Configuration I to Configuration II change. For each example, excellent prediction of mean errors and bounds on parameters critical to control design on the system was shown.

5. The final contribution follows from the utilization of the uncertainty model for control design. Four methods of this utilization are presented, two of which were demonstrated during the MACE flight. In addition, the prediction of the 0-g uncertainty model for MACE was one of the significant contributing factors in the design and evaluation of over 200 pre-programmed controllers which were stable on-orbit.

\subsection{Recommendations}

1. Although the summary of model uncertainties given in this work is one of the most thorough of its kind, because the uncertainties are considered stochastic, more work needs to be done in examining how these uncertainties manifest themselves into the model. Specifically, how the modeling uncertainties such as discretization errors and incorrect element physics, and nonlinearities manifest themselves into the uncertainty model, and subsequent localization and projection is not known. It was assumed that these are small, or could be represented using mean error and variance uncertainties. An examination of a small order example with a weak nonlinearity, for instance, would add insight to the localization and projection methods, as well as adding confidence in their application to systems with such modeling uncertainties.

2. The modeling of gravity effects for MACE was shown to be suspect because the $1-\mathrm{g}$ to $0-\mathrm{g}$ transition of the model and data were significantly different. A more thorough examination of the modeling of these effects should be done. 
This probably means a more thorough set of open loop test, i.e. more sensors. More sensor give more information on the mode shapes, and therefore better model updating and uncertainty localization. Additional sensors such as accelerometers on the nodes and suspension cables would give more insight into the modeling of the gravity effects, and update of the finite element model.

3. The Discrete Extended Kalman Filter (DEKF) was used to estimate the parameters of the system, and therefore identify the modal. Although an identification technique is needed which parameterizes the finite element model and actuator/sensor dynamics and time delays separately, the DEKF is not necessarily the best choice. For instance, there are no guarantees against bias errors. An examination of the parameter estimation techniques is needed to create the best uncertainty model. This can only be done using a detailed examination of the benefits and drawbacks of each correctly parameterized identification technique.

4. With a retesting of the MACE hardware, another examination of the methods localization and projection methods can be made using the additional sensors. In addition, the iterative procedure suggested for systems with larger errors can be employed in order to accurately identify the model errors. This requires a sparse matrix eigenvalue solver which can handle large systems (greater than 500 degrees of freedom), and rigid body modes.

5. The practical iterative procedure for localizing the mean errors in a finite element model needs to be examined for the localization of variance uncertainties also. This requires the solution of a nonlinear statistics problem which is quite difficult.

6. The final recommendation involves the use of the uncertainty model in control design. Controller synthesis and analysis techniques which use an uncertainty model usually design and analyze a controller for all possible perturbations of the uncertainty model. If the physical basis of the uncertainty model is a lower rank, however, this may be overly conservative. For instance, if there is an 
error in the density of one finite element, all of the frequencies and mode shapes change. Controller synthesis and analysis techniques will usually design for all perturbations of the uncertainty model. This is quite conservative, however, because there is only one perturbation. Additional work should be done in attempting to reduce this uncertainty model, based on the physical mass and stiffness uncertainties. 


\section{References}

[1] Ewins, D. J., Modal Testing: Theory and Practice, John Wiley and Sons, Inc, New York, NY, 1984.

[2] Ljung, L., System Identification: Theory for the User, Prentice-Hall, Inc., 1987.

[3] Craig, R. R. and Blair, M. A. C., "A Generalized Multiple-Input Multiple-Output Modal Parameter Estimation Algorithm," AIAA Journal, Vol. 23, No. 6, 1994, pp. 931-937.

[4] Lee, H. G. and Dobson, B. J., "The Direct Measurement of Structural Mass, Stiffness, and Damping Properties," Journal of Sound and Vibration, Vol. 145, No. 1, 1991, pp. 61-81.

[5] Balmes, E., Experimental/Analytical Predictive Models of Damped Structural Dynamics, Ph.D. thesis, Department of Aeronautics and Astronautics, M.I.T., Cambridge, MA, May 1993. MIT SERC report \# 7-93.

[6] Karlov, V. I., Miller, D. W., Vander Velde, W. E., and Crawley, E. F., "Identification of Model Parameters and Associated Uncertainties for Robust Control Design," AIAA Journal of Guidance, Control, and Dynamics, Vol. 17, No. 3, May.-June 1994, pp. 495-504.

[7] Caesar, B., "Updating System Matrices Using Model Test Data," Proceedings, the International Modal Analysis Conference (IMAC), 1987.

[8] Link, M., "Identification and Correction of Errors in Analytical Models Using Test Data - Theoretical and Practical Bounds," Proceedings, International Modal Analysis Conference, 1990, pp. 570-578.

[9] Maia, N. M. M., Reynier, M., and Ladeveze, P., "Error Localization for Updating Finite Element Models Using Frequency Response Functions," Proceedings, International Modal Analysis Conference, 1994, pp. 1299-1308.

[10] Lieven, N. A. J. and Ewins, D. J., "Correlation of Mode Shapes, the Coordinate Modal Assurance Criterion," Proceedings, International Modal Analysis Conference, 1988 , pp. 690-698. 
[11] Lieven, N. A. J. and Waters, T. P., "Error Location Using Normalised Orthogonality," Proceedings, International Modal Analysis Conference, 1994, pp. 761764 .

[12] Lin, C. S., "Location of Modeling Errors Using Modal Test Data," AIAA Journal, Vol. 28, No. 9, September 1990.

[13] Baruch, M. and Bar Itzhack, I. Y., "Optimal Weighted Orthogonalization of Measured Modes," AIAA Journal, Vol. 16, Apr. 1978, pp. 346-351.

[14] Baruch, M., "Optimization Procedure to Correct Stiffness and Flexibility Matrices Using Vibration Tests," AIAA Journal, Vol. 16, Apr. 1978, pp. 1208-1210.

[15] Berman, A. and Nagy, E. J., "Improvement of a Large Analytical Model Using Test Data," AIAA Journal, Vol. 21, No. 8, June 1983, pp. 1168-1173.

[16] Sidhu, J. and Ewins, D. J., "Correlation of Finite Element and Modal Test Studies of a Practical Structure," Proceedings, International Modal Analysis Conference, 1984, pp. 756-762.

[17] Guyan, R. J., "Reduction of Mass and Stiffness Matrices," AIAA Journal, Vol. 2, No. 1, Feb. 1965.

[18] Gysin, H., "Critical Application of the Error Matrix Method for Localisation of Finite Element Modeling Innacuracies," Proceedings, International Modal Analysis Conference, 1986, pp. 1339-1351.

[19] Natke, H. G., "Indirect Methods: Correction of the Results of Systems Analysis by Results of Identification - A Survey," Identification of Vibrating Structures, 1982, pp. 225-256.

[20] O'Callahan, J. C., "Determination of Analytical Model Differences Using Modal Updating," Proceedings, International Modal Analysis Conference, 1990, pp. 1180-1189.

[21] Ibrahim, S. R., Stavrinidis, C., Fissette, E., and Brunner, O., "A Direct Two Response Approach for Updating Analytical Dynamic Models of Structures with Emphasis on Uniqueness," Proceedings, International Modal Analysis Conference, 1989, pp. 340-346.

[22] Luber, W. and Lotze, A., "Application of Sensitivity Methods for Error Localization in Finite Element Systems," Proceedings, International Modal Analysis Conference, 1990, pp. 598-604.

[23] Lallement, G., "Indirect Identification Methods I: Adjustment of Methematical Models by the Results of Vibrating Tests: Using Eigensolutions," Identification of Vibrating Structures, 1982, pp. 179-194. 
[24] Zhang, Q., Lallement, G., Fillod, R., and Piranda, J., "A Complete Procedure for the Adjustment of a Mathematical Model from Identified Complex Modes," Proceedings, International Modal Analysis Conference, 1987, pp. 1183-1190.

[25] Lallement, G. and Piranda, J., "Localization Methods for Parametric Updating of Finite Element Models in Elastodynamics," Proceedings, International Modal Analysis Conference, 1990, pp. 579-585.

[26] Piranda, J., Lallement, G., and Cogan, S., "Parametric Correction of Finite Element Models by Minimization of an Output Residual: Improvement of the Sensitivity Method," Proceedings, International Modal Analysis Conference, 1991, pp. 363-368.

[27] Gordis, J. H., "An Exact Formulation for Structural Dynamic Model Error Localization," Proceedings, International Modal Analysis Conference, 1993, pp. 159167.

[28] He, J., "Sensitivity Analysis and Error Matrix Method Using Measured Frequency Resonse Function (FRF) Data," Proceedings, International Modal Analysis Conference, 1993, pp. 1079-1082.

[29] Fissette, E. Stavrinidis, C. and Ibrahim, S., "Error Location and Updating of Analytical Dynamic Models Using a Force Balance Method," Proceedings, International Modal Analysis Conference, 1989, pp. 1063-1070.

[30] Gysin, H., "Comparison of Expansion Methods for FE Modeling Error Localization," Proceedings, International Modal Analysis Conference, 1990, pp. 195-204.

[31] Collins, J. D., Hart, G. C., Hasselman, T. K., and Kennedy, B., "Statistical Identification of Structures," AIAA Journal, Vol. 12, No. 2, 1972, pp. 185-190.

[32] Hasselman, T. K. and Chrostowski, J. D., "Evaluation of Predictive Accuracy in Structural Dynamic Models," Proceedings, International Modal Analysis Conference, 1989, pp. 360-366.

[33] Hasselman, T. K., Chrostowski, J. D., and Ross, T. J., "Interval Prediction in Structural Dynamic Analysis," Proceedings, AIAA Structures, Structural Dynamics, and Materials Conference, Dallas, TX, Apr. 1992, pp. 1272-1284.

[34] Hasselman, T. K., Chrostowski, J. D., and Ross, T. J. C., "Propagation of Modeling Uncertainty through Structural Dynamic Models," Proceedings, AIAA Structures, Structural Dynamics, and Materials Conference, Hilton Head, SC, Apr. 1994.

[35] Crawley, E. F., Barlow, M. S., van Schoor, M. C., and Bicos, A. S., "Variation in the Modal Parameters of Space Structures," Proceedings, AIAA Structures, Structural Dynamics, and Materials Conference, Apr. 1992, pp. 1212-1228. 
[36] Crawley, E. F., Barlow, M. S., van Schoor, M. C., Masters, B. P., and Bicos, A. S., "Measurement of the Modal Parameters of a Space Structure in Zero Gravity," AIAA Journal of Guidance, Control, and Dynamics, Vol. 18, No. 3, May-June 1995, pp. 385-392.

[37] Miller, D. W., deLuis, J., Stover, G., How, J. P., Liu, K., Grocott, S. C. O., Campbell, M. E., Glaese, R., and Crawley, E. F., "The Middeck Active Control Experiment (MACE): Using Space for Technology Research and Development," Proceedings, American Control Conference, Seattle, WA, June 1995, pp. 397-401.

[38] Crawley, E. F., Masters, B. P., and Hyde, T. T., "Conceptual Design Methodology for High Performance Dynamic Structures," Proceedings, AIAA Structures, Structural Dynamics, and Materials Conference, New Orleans, LA, Apr. 1995.

[39] Glaese, R. M. and Miller, D. W., "Derivation of 0-g Structural Control Models from Analysis and 1-g Experimentation," Proceedings, AIAA Structures, Structural Dynamics, and Materials Conference, New Orleans, LA, Apr. 1995.

[40] Liu, K. and Miller, D. W., "Time Domain State Space Identification of Structural Systems," May 1993. Submitted to the ASME Journal of Dynamic Systems, Measurement, and Control.

[41] How, J. P., Glaese, R. M., Grocott, S. C. O., and Miller, D. W., "Finite Element Model Based Robust Controllers for the MIT Middeck Active Control Experiment (MACE)," Proceedings, American Control Conference, Baltimore, MD, June 1994, pp. 272-277.

[42] How, J. P. and Miller, D. W., "On-orbit Closed-loop Performance Predictions for the Middeck Active Control Experiment (MACE)," Proceedings, 1994 IFAC Symposium on Automatic Control in Aerospace, Palo Alto, CA, Sept. 1994, pp. 228-233.

[43] Liu, K. and Glaese, R., "On-orbit Modeling and System Identification for MACE," Proceedings, IFAC $13^{\text {th }}$ World Congress, San Francisco, CA, July 1996.

[44] Campbell, M. E., Grocott, S. C. O., and How, J. P., "Overview of Closed Loop Results for MACE," Proceedings, IFAC $13^{\text {th }}$ World Congress, San Francisco, CA, July 1996.

[45] Grocott, S. C. O. and Campbell, M. E., "Control Analysis Results for MACE: Methods and Limitations," Proceedings, IFAC $13^{\text {th }}$ World Congress, San Francisco, CA, July 1996.

[46] Grocott, S. C. O., How, J. P., Miller, D. W., MacMartin, D. G., and Liu, K., "Robust Control Implementation on the Middeck Active Control Experiment (MACE)," AIAA Journal of Guidance, Control, and Dynamics, Vol. 17, No. 6, Nov.-Dec. 1994, pp. 1163-1170. 
[47] Jacques, R. N. and Miller, D. W., "Multivariable Model Identification from Frequency Response Data," Proceedings, IEEE Conference on Decision and Control, San Antonio, TX, Dec 1993, pp. 3046-3051.

[48] Campbell, M. E., Grocott, S. C. O., How, J. P., Miller, D. W., and Crawley, E. F., "Verification Procedure for On-orbit Controllers for the Middeck Active Control Experiment," Proceedings, 1995 American Control Conference, Seattle, WA, June 1995, pp. 3600-3605.

[49] Glaese, R., Development of 0-gravity Structural Control Models from Ground Analysis and Experimentation, Master's thesis, Department of Aeronautics and Astronautics, M.I.T., Cambridge, MA, Jan. 1994.

[50] Glaese, R. and Miller, D. W., "On-orbit Modelling of the Middeck Active Control Experiment from 1-g Analysis and Experimentation," Proceedings, the 12th International Modal Analysis Conference (IMAC), Honolulu, Feb. 1994.

[51] Anderson, E. H., Piezoceramic Actuation of One- and Two-Dimensional S, Master's thesis, Department of Aeronautics and Astronautics, M.I.T., Cambridge, MA, Mar. 1989. MIT SSL report \# 5-89.

[52] Tiffany, S. H. and Jr., W. M. A., "Aeroservoelastic modeling and applications using minimum-state approximations of the unsteady aerodynamics," Proceedings, AIAA Structures, Structural Dynamics, and Materials Conference, Mobile, AL, Apr. 1989.

[53] Lin, C. Y., "Open Loop and Preliminary Closed Loop Results of a Strain Actuated Active Aeroelastic Wing," Proceedings, AIAA Structures, Structural Dynamics, and Materials Conference, Apr. 1995.

[54] Wada, B. K., "Extension of Ground-Based Testing for Large Space Structures," Proceedings, AIAA Structures, Structural Dynamics, and Materials Conference, Apr. 1985, pp. 477-483.

[55] Rey, D. A., Gravity and Laboratory Suspension Effects on Dynamics of Controlled Flexible Spacecraft, Master's thesis, Massachusetts Institute of Technology, 1993. MIT SERC report \# 6-92.

[56] Rey, D. A., Crawley, E. F., Alexander, H. L., Glaese, R. M., and Gaudenzi, P., "Gravity and Suspension Effects on the Dynamics of Controlled Structures," Proceedings, AIAA Structures, Structural Dynamics, and Materials Conference, (La Jolla, CA), Apr. 1993.

[57] Gelb, A., Applied Optimal Estimation, MIT Press, Cambridge, MA, 1974.

[58] Franklin, G. F., Powell, J. D., and Workman, M. L., Digital Control of Dynamic Systems, Addison-Wesley, 1990. 
[59] Ljung, L., "Asymptotic Behavior of the Extended Kalman Filter as a Parameter Estimator for Linear Systems," IEEE Trans. on Automatic Control, Vol. AC-24, No. 1, Feb. 1979, pp. 36-50.

[60] Ljung, L., "Analysis of Linear, Recursive, Stochastic Algorithms," IEEE Trans. on Automatic Control, Vol. AC-22, No. 1, Aug. 1977, pp. 551-575.

[61] Mutambara, A. G., Decentralized Estimation and Control with Applications to a Modular Robot, Ph.D. thesis, University of Oxford, 1995.

[62] Mutambara, A. G. and Durrant-Whyte, H. F., "Estimation and Information Space," Proceedings, IFAC $13^{\text {th }}$ World Congress, San Francisco, CA, July 1996.

[63] Eldred, M. S., Lerner, P. B., and Anderson, W. J., "Higher Order Eigenpair Perturbations," AIAA Journal, Vol. 30, No: 7, July 1992, pp. 1870-1875.

[64] Graham, A., Kronecker Products and Matrix Calculus: with Applications, Ellis Horwood Limited, 1981.

[65] Bathe, K.-J., Finite Element Procedures in Engineering Analysis, Prentice-Hall, Inc, Englewood Cliffs, NJ, 1982.

[66] MacNeal-Schwendler Corporation, NASTRAN Users Manual Version 67. Los Angeles, 1993.

[67] Doyle, J. C., Glover, K., Khargonekar, P. P., and Francis, B. A., "State-Space Solutions to Standard $\mathcal{H}_{2}$ and $\mathcal{H}_{\infty}$ Control Problems," IEEE Trans. on Automatic Control, Vol. AC-34, No. 8, Aug. 1989, pp. 831-847.

[68] Haddad, W. M. and Bernstein, D. S., "Generalized Riccati Equations for the Full- and Reduced-order Mixed-norm $\mathcal{H}_{2} / \mathcal{H}_{\infty}$ Standard Problem," Systems and Control Letters, Vol. 14, 1990, pp. 185-197.

[69] Doyle, J. C., "Structured Uncertainty in Control System Design," Proceedings, IEEE Conference on Decision and Control, Ft. Lauderdale, FL, 1985.

[70] Fan, M. K. H., Tits, A. L., and Doyle, J. C., "Robustness in the Presence of Mixed Parametric Uncertainty and Unmodeled Dynamics," IEEE Trans. on Automatic Control, Vol. AC-36, No. 1, Jan. 1991, pp. 25-38.

[71] Young, P. M., Newlin, M. P., and Doyle, J. C., " $\mu$ Analysis with Real Parametric Uncertainty," Proceedings, IEEE Conference on Decision and Control, Brighton, England, Dec. 1992, pp. 1251-1256.

[72] How, J. P. and Hall, S. R., "Connections between the Popov Stability Criterion and Bounds for Real Parameter Uncertainty," Proceedings, American Control Conference, San Francisco, June 1993, pp. 1084-1089. 
[73] How, J. P., Robust Control Design with Real Parameter Uncertainty use Absolute Stability Theory, Ph.D. thesis, Department of Aeronautics and Astronautics, M.I.T., Cambridge, MA, Jan. 1993.

[74] How, J. P., Hall, S. R., and Haddad, W. M., "Robust Controllers for the Middeck Active Control Experiment using Popov Controller Synthesis," IEEE Transactions on Control Systems Technology, Vol. 2, No. 2, June 1994, pp. 73-87.

[75] How, J. P., Collins, Jr., E. G., and Haddad, W. M., "Optimal Popov Analysis and Synthesis for Systems with Real Parameter Uncertainties," Dec 1995.

[76] Ashkenazi, A. and Bryson Jr., A. E., "Control Logic for Parameter Insensitivity and Disturbance Attenuation," AIAA Journal of Guidance, Control, and Dynamics, Vol. 5, No. 4, July 1982, pp. 383-388.

[77] MacMartin, D. G., Hall, S. R., and Bernstein, D. S., "Fixed Order Multi-Model Estimation and Control," Proceedings, American Control Conference, Boston, MA, June 1991, pp. 2113-2118.

[78] Hyland, D. C., "Maximum Entropy Stochastic Approach to Controller Design for Uncertain Structural Systems," Proceedings, American Control Conference, Arlington, VA, June 1982, pp. 680-688.

[79] Bernstein, D. S. and Hyland, D. C., "Maximum Entropy-type Lyapunov Functions for Robust Stability and Performance Analysis," Proceedings, American Control Conference, June 1992.

[80] Blelloch, P. A. and Mingori, D. L., "Robust Linear Quadratic Gaussian Control for Flexible Structures," AIAA Journal of Guidance, Control, and Dynamics, Vol. 13, No. 1, Jan. 1990, pp. 66-72.

[81] Sesak, J. R. and Likins, D. D., "Model Error Sensitivity Suppression: Quasistatic Optimal Control of Flexible Structures," Proceedings, IEEE Conference on Decision and Control, Ft. Lauderdale, FL, Dec. 1988.

[82] Wie, B. and Byun, K.-W., "New Generalized Structural Filtering Concept for Active Vibration Control Synthesis," AIAA Journal of Guidance, Control, and Dynamics, Vol. 12, No. 2, Mar.-Apr. 1989, pp. 147-154.

[83] Wie, B. and Gonzalez, M., "Control Synthesis for Flexible Space Structures Excited by Persistent Disturbances," AIAA Journal of Guidance, Control, and Dynamics, Vol. 15, No. 1, Jan.-Feb. 1992, pp. 73-80.

[84] Campbell, M. E., Neo-Classical Control of Structures, Master's thesis, Department of Aeronautics and Astronautics, M.I.T., Cambridge, MA, Feb. 1993. MIT SERC report \# 4-92. 
[85] Campbell, M. E. and Crawley, E. F., "Classically Rationalized Low Order Robust Structural Controllers," Proceedings, $35^{\text {th }}$ Structures, Structural Dynamics and Materials Conference, Hilton Head, SC, Apr. 1994.

[86] D'Azzo, J. J. and Houpis, C. H., Linear Control System Analysis and Design: Conventional and Modern, McGraw-Hill Inc., New York, 1988.

[87] Kharitonov, V. L., "Asymptotic Stability of an Equilibrium Position of a Family of Systems of Linear Differential Equations," Differencial'nye Uravenija, Vol. 14, No. 11,1978 , pp. 2086-2088.

[88] Grocott, S. C. O., How, J. P., and Miller, D. W., "Comparison of Control Techniques for Robust Performance on Uncertain Structural Systems," Proceedings, AIAA Guidance, Navigation, and Control Conference, Scottsdale, AZ, Aug. 1994, pp. 261-271.

[89] Morton, B. G. and McAfoos, R. M., "A Mu-Test for Robustness Analysis of Real Parameter Variation Problem," Proceedings, American Control Conference, May 1985, pp. 135-138.

[90] Grocott, S., Comparison of Control Techniques for Robust Performance on Uncertain Structural Systems, Master's thesis, Massachusetts Institute of Technology, 1994.

[91] Kwakernaak, H. and Sivan, R., Linear Optimal Control Systems, WileyInterscience, 1972.

[92] Sesak, J. R., Sensitivity Constrained Linear Optimal Control Analysis and Synthesis, Ph.D. thesis, University of Wisconsin, 1974.

[93] Grocott, S. C. O., 1995. Personal Communication.

[94] Tahk, M. and Speyer, J. L., "Modeling of Parameter Variations and Asymptotic LQG Synthesis," IEEE Trans. on Automatic Control, Vol. AC-32, No. 9, Sept. 1987, pp. 793-801. 